\title{
Optics of Conducting Polymer Thin Films and \\ Nanostructures
}

\author{
Disertation No. 2107
}





\title{
Optics of Conducting Polymer Thin Films and Nanostructures
}

\author{
Shangzhi Chen
}

(陈尚志)

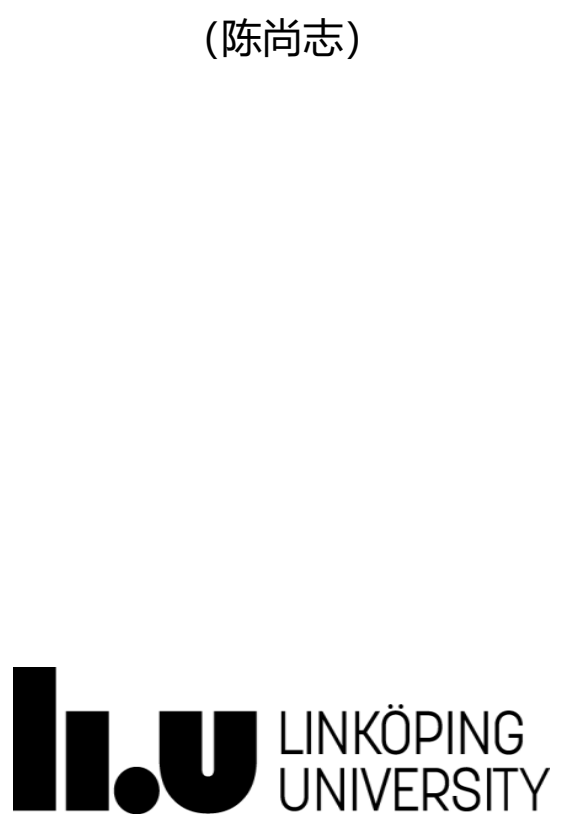

Department of Science and Technology

Linköping University, Sweden

Norrköping 2021 


\section{Description of the cover image:}

The illustration of the cover represents conducting polymer nano-antennas and structural colour devices made of PEDOT-based materials. It was based on an artwork designed by Dr. Robert Brooke (conceptualized.tech) and modified by Shangzhi Chen.

(c) EY-NC This work is licensed under a Creative Commons AttributionNonCommercial 4.0 International License.

https://creativecommons.org/licenses/by-nc/4.0/

Optics of Conducting Polymer Thin Films and Nanostructures

Copyright @ Shangzhi Chen, 2021

During the course of the research underlying this thesis, Shangzhi Chen was enrolled in the graduate school Agora Materiae, a multidisciplinary doctoral program at Linköping University, Sweden

ISBN 978-91-7929-745-9

ISSN 0345-7524

Printed by LiU-Tryck, Linköping, Sweden, 2021 
Dedicated to my family, especially my dear parents and grandparents 

凡操干曲而后晓声，观干剑而后识器；故圆照之象，务先博观。阅乔岳以形培塿，酌沧 波以喻洇浍。无私于轻重，不偏于憎爱，然后能平理若衡，照辞如镜矣。

一刘触《文心雕龙知音第四十八》 



\section{Abstract}

Intrinsically conducting polymers forms a category of doped conjugated polymers that can conduct electricity. Since their discovery in the late 1970s, they have been widely applied in many fields, ranging from optoelectronic devices to biosensors. The most common type of conducting polymers is poly(3,4-ethylenedioxythiophene), or PEDOT. PEDOT has been popularly used as electrodes for solar cells or lightemitting diodes, as channels for organic electrochemical transistors, and as p-type legs for organic thermoelectric generators. Although many studies have been dedicated to PEDOT-based materials, there has been a lack of a unified model to describe their optical properties across different spectral ranges. In addition, the interesting optical properties of PEDOT-based materials, benefiting from its semimetallic character, have only been rarely studied and utilized, and could potentially enable new applications.

Plasmonics is a research field focusing on interactions between light and metals, such as the noble metals (gold and silver). It has enabled various opportunities in fundamental photonics as well as practical applications, varying from biosensors to colour displays. This thesis explores highly conducting polymers as alternatives to noble metals and as a new type of active plasmonic materials. Despite high degrees of microstructural disorder, conducting polymers can possess electrical conductivity approaching that of poor metals, with particularly high conductivity for PEDOT deposited via vapour phase polymerization (VPP). In this thesis, we systematically studied the optical and structural properties of VPP PEDOT thin films and their nanostructures for plasmonics and other optical applications.

We employed ultra-wide spectral range ellipsometry to characterize thin VPP PEDOT films and proposed an anisotropic Drude-Lorentz model to describe their optical conductivity, covering the ultraviolet, visible, infrared, and terahertz ranges. Based on this model, PEDOT doped with tosylate (PEDOT:Tos) presented negative real permittivity in the near infrared range. While this indicated optical metallic character, the material also showed comparably large imaginary permittivity and associated losses. To better understand the VPP process, we carefully examined films with a collection of microstructural and spectroscopic characterization methods and found a vertical layer stratification in these polymer films. We unveiled the cause as related to unbalanced transport of polymerization precursors. By selection of suitable counterions, e.g., trifluoromethane sulfonate (OTf), and optimization of reaction conditions, we were able to obtain PEDOT films with electrical conductivity exceeding $5000 \mathrm{~S} / \mathrm{cm}$. In the near infrared range from 1 to $5 \mu \mathrm{m}$, these PEDOT:OTf films provided a well-defined plasmonic regime, characterized by negative real permittivity and lower magnitude imaginary component. Using a colloidal lithography-based approach, we managed to fabricate nanodisks of PEDOT:OTf and showed that they exhibited clear plasmonic absorption features. The experimental results matched theoretical calculations and numerical simulations. Benefiting from their mixed ionicelectronic conducting characters, such organic plasmonic materials possess redox- 
tunable properties that make them promising as tuneable optical nanoantennas for spatiotemporally dynamic systems. Finally, we presented a low-cost and efficient method to create structural colour surfaces and images based on UV-treated PEDOT films on metallic mirrors. The concept generates beautiful and vivid colours throughout the visible range utilizing a synergistic effect of simultaneously modulating polymer absorption and film thickness. The simplicity of the device structure, facile fabrication process, and tunability make this proof-of-concept device a potential candidate for future low-cost backlight-free displays and labels. 


\section{Populärvetenskaplig sammanfattning}

Ledande polymerer är en typ av dopade konjugerade polymerer som kan leda elektricitet. Sedan de upptäcktes på 1970-talet har de använts inom många olika områden, från solceller till biosensorer. Poly(3,4-etylendioxietiofen), eller PEDOT, är den mest använda ledande polymeren. PEDOT har intressanta egenskaper som gjort polymeren populär i organiska solceller, ljusdioder, elektro-kemiska transistorer och termoelektriska generatorer. Även om många studier har ägnats åt PEDOT-baserade material så har det saknats en enhetlig modell för att beskriva deras optiska egenskaper $\mathrm{i}$ ett brett våglängdsspann. Vidare är de optiska egenskaperna hos PEDOT-baserade material fortfarande relativt outforskade och det finns möjligheter till nya upptäckter och applikationer.

Inom området plasmonik används filmer och nanostrukturer av metaller (såsom guld och silver) för att kontrollera ljus på olika sätt, vilket möjliggjort allt från nya typer av biosensorer till färgskärmar. I vår forskning har vi undersökt ledande polymerer som alternativ till mer traditionella plasmoniska metaller och som en ny typ av aktiva plasmoniska material. Den elektriska ledningsförmågan hos ledande polymerer, särskilt PEDOT-baserade filmer framställda genom ångfaspolymerisering, har ökat kraftigt de senaste åren och närmar sig ledningsförmågan hos vissa traditionella metaller. I den här avhandlingen har vi studerat de optiska och strukturella egenskaperna hos tunna filmer och nanostrukturer av PEDOT, och undersökt dess användning som styrbara optiska antenner och för att generera strukturella färger.

Vi använde ellipsometri för att karakterisera tunna PEDOT-filmer och utvecklade en modell för att beskriva deras optiska egenskaper över ett brett våglängdsområde, som täcker in ultraviolett, synligt och infrarött ljus och även terahertz-området. Det gjorde det möjligt att visa att PEDOT dopat med p-toluensulfonat (PEDOT:Tos) kan ha en reell permittivitet som är negativ i det närinfraröda området, vilket betyder att materialet fungerar optiskt som en metall. Materialet hade dock relativt hög imaginär permittivitet, vilket leder till absorption och dämpning när det används till optiska tillämpningar. Utöver optiska och elektriska egenskaper har vi även studerat strukturella egenskaper hos tunna PEDOT-filmer framställda genom ångfaspolymerisering. Vi upptäckte en vertikal inhomogenitet hos filmerna och föreslog att den beror på obalanserad transport av material under polymeriseringsprocessen.

Genom att byta motjon från Tos till trifluormetansulfonat (OTf) kunde vi tillverka PEDOT-filmer med elektrisk ledningsförmåga över $5000 \mathrm{~S} / \mathrm{cm}$. Det gav filmer med egenskaper som gör dem intressanta som plasmoniska material i det närinfraröda området. Vi lyckades tillverka nanodiskar av materialet och visa att de kan användas som optiska nanoantenner med tydliga plasmoniska absorptionsresonanser. De experimentella resultaten stämde bra med teoretiska beräkningar och numeriska simuleringar. Vi visade sedan att antennerna kunde stängas av och sättas på igen genom att styra polymerens ledningsförmåga på kemisk väg. Sådana styrbara nanoantenner för ljus har potential att användas inom många olika områden och tillämpningar. Slutligen presenterar vi möjligheten att skapa styrbara färgade ytor och bilder genom en UV-mönstringsprocess av PEDOT på reflekterande metallytor. 
Konceptet kunde generera vackra reflekterande färger, vilket förklarades av en synergistisk effekt av gradvis förändring av både tjocklek och polymerabsorption. Enkelheten i konceptet och möjligheten till styrbarhet gör det till en potentiell kandidat för nästa generations energieffektiva bakgrundsbelysningsfria skärmar och etiketter. 


\section{Acknowledgements}

I started my Ph.D. from 2016 and the nice journey is now coming to an end. The fouryear study and research experience at LOE would be the most important part of my life. It is my great honour to be a member of the lab and meet so many wonderful and talented scientists here. There is an ultra-long list of people that I should acknowledge for teaching and helping me in overcoming challenges. Without you, it was impossible for me to reach this far.

First and foremost, I would like to express my deepest gratitude to Magnus Jonsson, my supervisor, for offering me the opportunity and trust to explore such a brand-new field. It is your patient guidance, enthusiastic encouragement, and consistent support that made everything possible and achievable. I can always learn and get inspired from discussions with you, not only on fundamental knowledge and experimental techniques but also on critical thinking and writing. I really appreciate your unreserved support on my research ideas even if they were strongly deviating from the focus of my Ph.D. thesis. I also want to thank you for nominating or recommending me as invited speaker for national and international conferences, which benefited my career. It is an amazing experience to have you as my supervisor and the years working with you will be the best memory of my life.

I am also grateful to my co-supervisor, Vanya Darakchieva, for your great guidance, unreserved support, and insightful advices throughout my Ph.D. Your optimism and enthusiasm towards scientific research inspire me a lot. I learned a lot from you, not only on the right way to excellent research, but also on leadership, team-building, and life-work balance. I appreciate being a part of your team at THeMAC.

I would like to thank my outstanding collaborators, Philipp Kühne, Mathias Schubert, Vallery Stanishev, loannis (Yiannis) Petsagkourakis, Eleni Pavlopoulou, Hengda Sun, Nicoletta Spampinato, Xianjie Liu, Nerijus Armakavicius, Sean Knight, and Steffen Richter, for delivering your knowledge and experiences. Your invaluable expertise in ellipsometry and conducting polymers significantly enhanced the quality of our study.

The thesis could not be a reality without the support from the Organic Photonics and Nano-Optics team. I would like to thank Evan for guiding me when I first came to the lab, and advices on my studies, research projects, and careers; Mina for helping me deal with problems in both course studies and experiments; Daniel for organizing the nice workshop on numerical simulations; Ravi, Stefano, Samim, Sampath for teaching me on equipments and techniques used in the photonics lab; Giancarlo, Debashree, Akchheta and Subham for supporting me with experiments, simulations, and sharing expertise and knowledge that are useful for our projects. It is great to see that our team is growing bigger and stronger.

PHOT ${ }^{4}$ 'NICS \& NAN 4 PTICS

I would like to acknowledge Xavier Crispin, Mats Fahlman, Simone Fabiano, Magnus Berggren, Isak Engquist, Igor Zozoulenko, Thomas Ederth, Olle Inganäs, and Martijn 
Kemerink for those highly useful, inspiring discussions and advices on my research, potential side projects, and showing me the beauty of different research fields.

There are plenty of highly useful Ph.D. courses offered at Linköping University and I am greatly benefited from them. I would like to thank these course instructors: Daniel Simon, Eric Glowacki, Klas Tybrandt, Eleni Stavrinidou, Martijn Kemerink, Wei-Xin $\mathrm{Ni}$, Hans Arwin, Roger Magnusson, Sergiy Valyukh, Mikhail Vagin, Victor Gueskine, Jian-Wu Sun, Carlito Ponseca, and Xiao-Ke Liu. I appreciate your efforts in preparing these lectures and answering all my (stupid and naive) questions.

I would like to thank the lab crew team, Lars Gustavsson, Thomas Karlsson, Meysam Karami Rad, Anna Malmström, and Jenny Joensuu, for providing a fantastic and outstanding lab working environment and tremendous help with all technical issues in the cleanroom. I would like to thank the administrative team: Ami Palmin, Lesley G Bornhöft, Kattis Nordlund, Martina Klefbeck, Jenny Nerkell, Katarina Swanberg, and Annelie Westerberg, for the great support on contract, visa applications, individual study plans, and all other financial stuffs. I also want to say thanks to Jens Isacsson and Tobias Svensson for the excellent IT support, and Thor Balkhed for these beautiful pictures and photos on our materials and devices.

I would like to thank my colleagues at LOE and RISE: Robert Brooke, Jesper Edberg, Kosala Wijerantne, Zia Ullah Khan, Tero-Petri Ruoko, Nara Kim, Jennifer Gerasimov, Mehmet Girayhan Say, Sarbani Ghosh, Arman Molaei, Hamid Ghorhani Shiraz, Tran van Chinh, Johannes Gladisch, Arghyamalya Roy, Fatima Nadia Ajjan, Gwennael Dufil, Mohsen Mohammadi, Oliya Abdullaeva, Tobias Abrahamsson, Maciej Gryszel, Josefin Nissa, Ujwala Ail, Makara Lay, Ayesha Sultana, David Poxson, Ziyauddin Khan, Gabor Mehes, Samuel Lienemann, Ludovico Migliaccio, Fareed Ahmed, Xin Wang, Maria Seitanidou, Marie Jakesova, Xenofon Strakosas, and Nitin Wadnerkar for practical discussion and advices on improving my experiments and collaborations. I want to say thank you to Evangelia Mitraka for giving me the opportunity to organize LOE Friday Meeting, a challenging task at first but finally a valuable experience to me. I also want to thank Dennis Cherian and Changbai Li for taking over this Friday responsibility. In addition, I want to thank Marzieh Zabihipour, Eva Miglbauer, and Anna Håkansson, for ordering chemicals, which are critical for all our studies.

I would like to thank these amazing people from Agora Materiae Graduate School, especially Caroline Brommesson, Per Olof Holtz, Fredrik Karlsson, and Wendela Yonar for organizing those incredible study visits, annual conferences, and monthly research seminars (Fika and cakes). I want to thank Binbin Xin for great collaboration in organizing the 2020 fall Ph.D. conference; Jianwei Yu, Yanfeng Liu, Yingzhi Jin, Nannan Yao, Dunyong Deng, Elfatih Mohammed Mustafa, Pimin Zhang, Quanzhen Tao, Nasrin Razmi, Hengfang Zhang, Rui Shu, Hatim Alnoor, Rania Elhadi Adam, Davide Gambino, Zhongcheng Yuan, Hongling Yu, Yuming Wang, Heyong Wang, Huotian Zhang, and Fuxiang Ji for all beneficial discussions on research, career, life, and funny things. 
I would like to thank my Chinese friends at LOE and Sweden: Hengda Sun, Fei Jiao, Dan Zhao, Kai Xu, Qiong Wu, Gang Wang, Shaobo Han, Canyan Che, Chuanfei Wang, Hongli Yang, Shengyang Zhou, Chi-Yuan Yang, Hanyan Wu, Jing-Xing Jian, Suhao Wang, Xiane Li, Zhixing Wu, Deyu Tu, Qingqing Wang, Junda Huang, Jiu Pang, Penghui Ding, Qilun Zhang, Silan Zhang, Yu Liu, Sheng Li, Xiaoyan Zhou, Xin $\mathrm{Xu}$, Renbo Wei, Lixin Mo, Yongzhen Chen, and Wenlong Jin, for sharing knowledge and experiences, offering help and spending happy times with me, including travelling and Chinese parties (BBQs, hot pots, Chinese New Year dinners, making dumplings, playing mahjong, poker, Werewolves of Millers Hollow, and other board games). It is impossible for me to get through the boring and hard times, especially the pandemic period, without you. I am so lucky and glad to meet and make friends with you all here in Sweden.

I would like to thank Kunt and Alice Wallenberg Jubileumsfond for providing me a traveling scholarship on 2020 MRS Spring conference, although I did not manage to attend it physically due to the annoying Covid-19 situation.

Finally, I would like to express my gratitude and appreciation to my family, especially my parents and grandparents, for allowing me to pursue my dreams abroad for such a long time. It is impossible for me to reach this far without your endless support and unconditional love. I want to thank Chaoyang for all the love and support, and being the only one to create these wonderful memories with me.

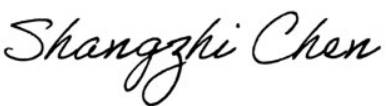

Norrköping, 2021 



\section{List of Publications}

Papers appended in the thesis:

1. On the anomalous optical conductivity dispersion of electrically conducting polymers: ultrawide spectral range ellipsometry combined with a Drude-Lorentz model

Shangzhi Chen, Philipp Kuhne, Vallery Stanishev, Sean Knight, Robert Brooke, loannis Petsagkourakis, Xavier Crispin, Mathias Schubert, Vanya Darakchieva, and Magnus P. Jonsson

Journal of Materials Chemistry C, 2019, 7, 4350.

Contribution: I fabricated the films and performed ellipsometry data analysis and other important characterizations. I wrote the first draft and contributed to the final editing of the manuscript.

2. Unravelling vertical inhomogeneity in vapour phase polymerized PEDOT:Tos films

Shangzhi Chen, loannis Petsagkourakis, Nicoletta Spampinato, Chaoyang Kuang, Xianjie Liu, Robert Brooke, Evan S. H. Kang, Mats Fahlman, Xavier Crispin, Eleni Pavlopoulou, and Magnus P. Jonsson Journal of Materials Chemistry A, 2020, 8, 18726.

Contribution: I prepared the films and performed data analysis and supplementary characterization. I wrote the first draft and contributed to the final editing of the manuscript.

3. Conductive polymer nanoantennas for dynamic organic plasmonics

Shangzhi Chen, Evan S. H. Kang, Mina Shiran Chaharsoughi, Vallery Stanishev, Philipp Kuhne, Hengda Sun, Chuanfei Wang, Mats Fahlman, Simone Fabiano, Vanya Darakchieva, and Magnus P. Jonsson

Nature Nanotechnology, 2020, 15, 35.

Contribution: I prepared the films, fabricated the nanostructures, carried out most characterizations and numerical simulations. I wrote the first draft and contributed to the final editing of the manuscript.

4. Redox-tunable structural colour images based on UV-patterned conducting polymers

Shangzhi Chen, Stefano Rossi, Ravi Shanker, Giancarlo Cincotti, Sampath Gamage, Philipp Kuhne, Vallery Stanishev, Isak Engquist, Magnus Berggren, Jesper Edberg, Vanya Darakchieva, and Magnus P. Jonsson

Submitted manuscript, 2021. (arXiv: 2101.08017)

Contribution: I was part in conceiving and designing the study and performed all the processes related to the device fabrication and data analysis, with assistance from S.R. and R.S. I wrote the first draft of the manuscript. 


\section{Additional publications not included in the thesis:}

- Strong plasmon-exciton coupling with directional absorption features in optically thin hybrid nanohole metasurfaces

Evan S. H. Kang, Shangzhi Chen, Samim Sardar, Daniel Tordera, Nerijus Armakavicius, Vanya Darakchieva, Timur Shegai, and Magnus P. Jonsson

ACS Photonics, 2018, 10, 4046.

- Electrical Transport Properties in PEDOT Thin Films

Nara Kim, loannis Petsagkourakis, Shangzhi Chen, Magnus Berggren, Xavier Crispin, Magnus P. Jonsson, and Igor Zozoulenko

Book chapter in the book "Conjugated Polymers: Properties, Processing, and Applications" (CRC Press Taylor \& Francis Group), 2019.

- Non-iridescent Biomimetic Photonic Microdomes by Inkjet Printing

Ravi Shanker, Samim Sardar, Shangzhi Chen, Sampath Gamage, Stefano Rossi, and Magnus P. Jonsson

Nano Letters, 2020, 20, 7243.

- Charge transport in phthalocyanine thin-film transistors coupled with FabryPerot cavities

Evan S. H. Kang, Shangzhi Chen, Vedran Derek, Carl Hägglund, Eric Glowacki, and Magnus P. Jonsson

Journal of Materials Chemistry C, 2021, in press. (DOI: 10.1039/D0TC05418F) 


\section{Contents}

\section{Part I Background}

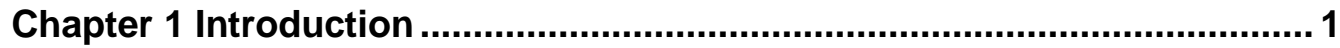

1.1 Manipulation of light-matter interactions ................................................ 1

1.2 Conducting polymers and their potential for nanooptics.......................... 2

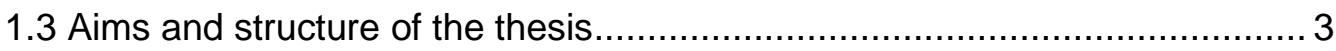

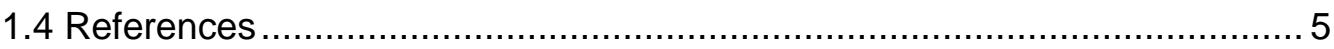

Chapter 2 Materials Optics ....................................................................... 7

2.1 Light as electromagnetic wave ............................................................. 7

2.2 Light-matter interactions .................................................................. 11

2.3 Optical models for materials ........................................................ 14

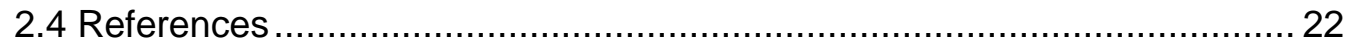

Chapter 3 Plasmonics and Structural Colours.......................................... 25

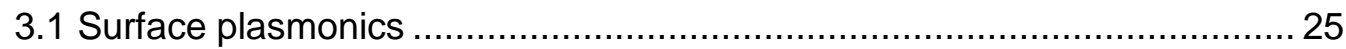

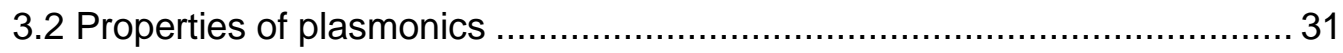

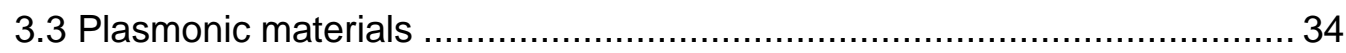

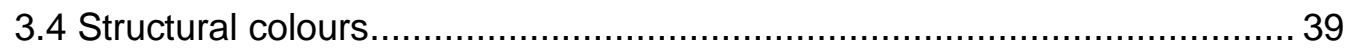

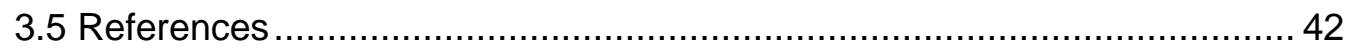

Chapter 4 Conducting polymers ............................................................... 47

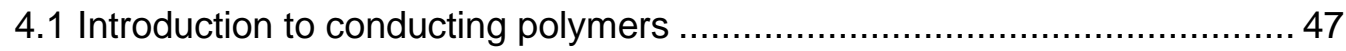

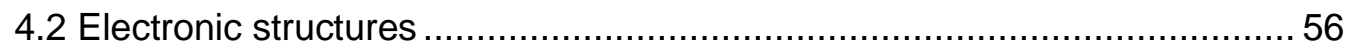

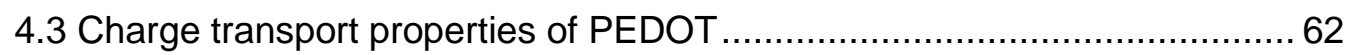

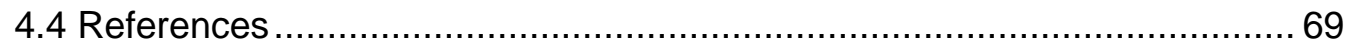

Chapter 5 Methods and Characterization ............................................... 75

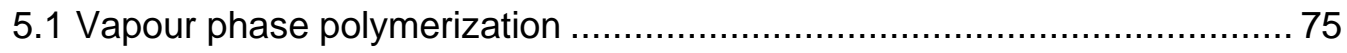

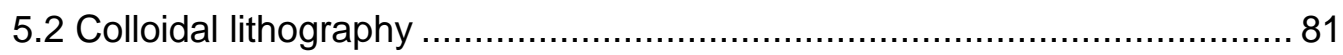

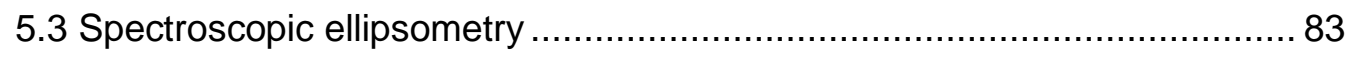

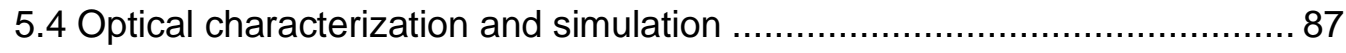

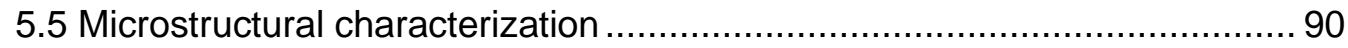

5.6 Other basic characterization methods................................................. 92

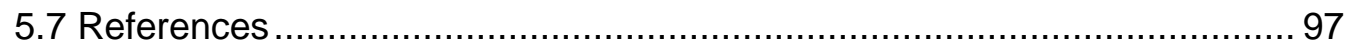

Chapter 6 Summary of Appended Papers ............................................... 103

6.1 Optical properties of PEDOT thin films (Paper 1)................................. 103 
6.2 Understanding vapour phase polymerization (Paper 2) ......................... 105

6.3 Switchable organic nanoantennas (Paper 3) ....................................... 107

6.4 Redox-tunable PEDOT structural colours (Paper 4) ............................... 109

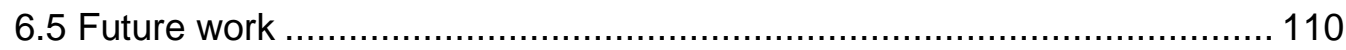

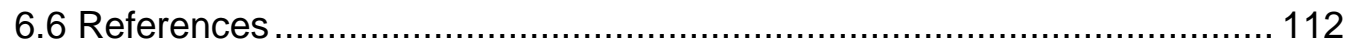

\section{Part II Scholarly Articles}

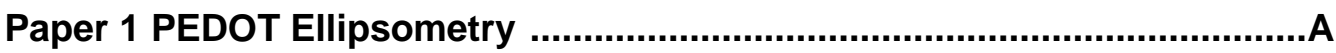

Paper 2 Vertical inhomogeneity in PEDOT films .......................................B

Paper 3 Conducting polymer nanoantennas .............................................

Paper 4 Redox-tunable structural colours of PEDOT .................................. 


\section{List of Abbreviations}

AFM Atomic force microscope

CB-54 Clevios product B-54 (oxidant)

CB Conduction band

CP Conducting polymers

CVD Chemical vapour deposition

DL Drude-Lorentz

DMSO Dimethyl sulfoxide

DS Drude-Smith

EBL Electron Beam Lithography

EDOT 3,4-ethylenedioxythiphene

EG Ethylene glycol

FDTD Finite-difference time-domain

FEM Finite Element Method

FIR Far infrared

FPC Fabry-Perot cavity

FTIR Fourier Transform infrared spectroscopy

GISAXS Grazing incidence small angle X-ray scattering

GIWAXS Grazing incidence wide angle

X-ray scattering

HOMO Highest occupied molecular orbital

iCP in-situ chemical polymerization

ITO Indium Tin Oxide

LMD Localization-modified Drude

LUMO Lowest unoccupied molecular orbital

LSPR Localized surface plasmon resonance

MIR Middle infrared

ND Nano-disk

NH Nano-hole

NIR Near infrared

NP Nano-particles

OM Optical microscope

OTf Trifluoromethane sulfonate

$\begin{array}{ll}\text { PANI } & \text { Polyaniline } \\ \text { PDDA } & \begin{array}{l}\text { Poly(diallyldimethylammonium } \\ \text { chloride) }\end{array} \\ \text { PEDOT } & \begin{array}{l}\text { Poly(3,4- } \\ \text { ethylenedioxythiphene) }\end{array} \\ \text { PEG } & \text { Poly(ethylene glycol) } \\ \text { PEI } & \text { Polyethylenimine } \\ \text { PG } & \text { Propylene glycol } \\ \text { PMMA } & \text { Poly(methyl methacrylate) } \\ \text { PPG } & \text { Poly(propylene glycol) } \\ \text { PPy } & \text { Polypyrrole } \\ \text { PS } & \text { Polystyrene } \\ \text { PSS } & \text { Polystyrene sulfonate } \\ \text { PTh } & \text { Polythiophene } \\ \text { SA } & \text { Self-assembly } \\ \text { SCL } & \text { Sparse Colloidal Lithography } \\ \text { SEM } & \text { Scanning Electron Microscopy } \\ \text { SPP } & \text { Surface plasmon polariton } \\ \text { SRO } & \text { Short-range order } \\ \text { Sulf } & \text { Sulfate } \\ \text { TEM } & \text { Transmission Electron } \\ \text { THz } & \text { Microscopy } \\ \text { TMM } & \text { Terahertz } \\ \text { Tos } & \text { Transfer Matrix Method } \\ & \text { Tosylate, or para- } \\ \text { UPS } & \text { Uluenesulfonate } \\ \text { UV } & \text { Spectroscopy } \\ \text { VASE } & \text { Vltraviolet } \\ \text { VB } & \text { Ellipsometry } \\ \text { VPP } & \text { Valence band } \\ \text { VRH } & \text { Variable range hopping } \\ \text { spectroscopy }\end{array}$



Part I

Background 


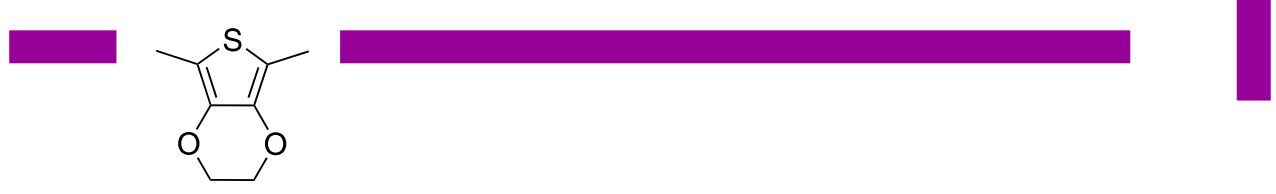

\section{Introduction}

This chapter presents background information and motivation to the work of this thesis. The main aim of the study was to develop a new type of dynamic nano-optical systems based on redox-tunable conducting polymers, meeting the demand of precise manipulation of light-matter interactions. The structure of this thesis will be described in the last section of the chapter.

\section{Contents}

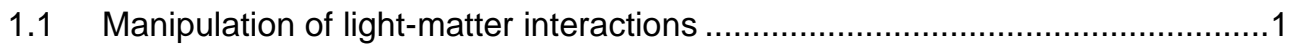

1.2 Conducting polymers and their potential for nano-optics...............................

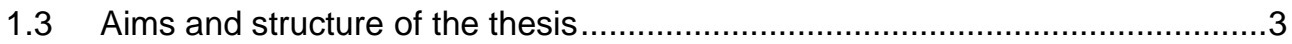

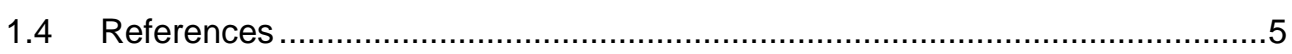

\subsection{Manipulation of light-matter interactions}

Light shapes the way we visualize the world by its interactions with all matters. Basic light-matter interactions ${ }^{1}$, such as absorption, reflection, scattering, transmission, and refraction, enable vast applications in our daily life ranging from mirrors and glasses to television monitors and optical fibers. With the development of nanotechnologies, researchers began to focus on the possibility of engineering light to desired properties at the nanoscale based on precise and accurate manipulation of light-matter interac- 
tions. Not least, metallic nanostructures emerged as a new category of nano-optical systems for this purpose, although they had been used for thousands of years to colour glass ${ }^{2}$. These nano-optical systems show great capability in precisely controlling light at the nanoscale, owing to the existence of plasmons which are defined as collective oscillations of free conduction electrons in metallic nanostructures ${ }^{3,4}$. Although their optical properties have been known for almost a century within the frame of classical electrodynamics, only several decades ago the advancements in new fabrication and characterization techniques enabled scientists and researchers to synthesize nano-structures of arbitrary geometry and measure their properties at the nanoscale.

Currently, plasmonic nanostructures made of coinage metals (e.g., gold or silver) have found lots of promising applications, including biosensing ${ }^{5}$, photocatalysis ${ }^{6}$, photonic management ${ }^{7}$, chemical reactions ${ }^{8}$, wireless communication ${ }^{9}$, nanofabrication ${ }^{10}$, and energy devices ${ }^{11}$. Although the vast majority of plasmonic systems have been based on conventional metals, these metallic nanostructures have posed certain limitations. Among them is the absence of tunability of their optical behavior since the properties of metals (e.g., permittivity) are fixed after fabrication. In turn, spatiotemporal tunability is considered as an important character for future nano-optical systems ${ }^{12}$.

Different strategies have been proposed attempting to eliminate or mitigate the issue, including tuning the refractive index of the surrounding media ${ }^{13}$, utilization of metal oxides and hydrides ${ }^{14}$, and exploitation of new conductors like graphene ${ }^{15}$. These methods can exhibit either long response times or poor reversibility and therefore might not be the best option for tunable plasmonic systems. Currently, the chase for active plasmonic materials with intrinsic tunability forms one of the main research directions in the plasmonics community.

\subsection{Conducting polymers and their potential for nano-optics}

Conducting polymers are defined as polymers that can conduct electricity, which were first reported in $1970 \mathrm{~s}^{16-18}$. Since their first discovery, conducting polymers have been widely utilized for a lot of electronic devices, ranging from optoelectronic and energy storage devices, to wearable bio-electronic systems ${ }^{19}$. The electrical conductivity in these polymers mostly originates from charge carriers generated by redox doping, where charge transfer occurs between the polymer and the dopant, leaving a positive or negative charged carrier on the backbone ${ }^{20}$. This doping process is fundamentally different from that in inorganic semiconductors like silicon or gallium arsenide, where doping is an irreversible process. For conducting polymers, their electrical conductivity can be altered in a broad range of more than 8 orders of magnitude by doping or dedoping ${ }^{18}$. The redox-state tuning in conducting polymers can be realized via various approaches, including chemical (vapour) treatment ${ }^{17,21}$ and electrochemical bias ${ }^{22,23}$. The timescale of such tuning for some systems can reach the order of microseconds ${ }^{24}$, favorable for high-speed switching. 
Similar to metals and transparent conductive oxides ${ }^{25}$, conducting polymers can have a high density of mobile charge carriers ${ }^{26,27}$, which can potentially oscillate collectively in response to light and enable the polymer to be used as a plasmonic material with tunable properties. However, the nature of these charge carriers is a bit different. The charge carriers, polarons or bipolarons, in conducting polymers are quasi-particles formed by coupling of electrons or holes and molecular vibrations (polymer backbone deformation) ${ }^{28}$. Compared to free electrons in metals, (bi-)polaronic carriers are more localized and sensitive to the microstructure of the polymer. This forms a challenge for plasmonic systems based on conducting polymers, at least for the understanding of the properties of (bi-)polarons and their possibilities for collective oscillations.

A way to potentially circumvent this challenge is to evaluate its optical parameters, or more specifically, permittivity (or optical conductivity) dispersion ${ }^{29}$. The permittivity can be extracted using ellipsometry combined with suitable optical model fitting. The previously dominating optical models for conducting polymers, the Locali-zation modified Drude model and the Drude-Smith model, are problematic and may derive incorrect results $^{27}$. Thus, developing a suitable optical model for conducting polymers formed one of the primary tasks of this thesis. The developed model should enable us to determine whether conducting polymers can possess plasmonic regimes or not. The exciting next task of this thesis was then to evaluate to what extent nanostructures made from these polymers could be utilized as switchable plasmonic nanoantennas, which included design of optimized nanostructures and fabrication techniques.

Conducting polymers have long been known to be suitable for electrochromism and some devices based on them are on the way towards commercialization ${ }^{30}$. However, the available colours based on polymer electrochromism is highly limited, such as light blue (transparent) to dark blue (opaque) for PEDOT-based materials ${ }^{31}$. Although multicolours could be realized via different polymers ${ }^{30}$, achieving various colours in one material is highly sought for. Structural colours, which exploit light-matter interactions ${ }^{32}$ induced by micro-/nanostructure instead of only material absorption, form a potential approach to achieve this goal, which was rarely studied for conducting polymers. Thus, another aim of this thesis was to study structural colours based on conducting polymer films and to create multi-colour images using simple and low-cost techniques.

In summary, conducting polymers with redox-state tunability show great promise for plasmonics although we have to overcome some existing challenges. Conducting polymer plasmonics could be advantageous for many applications, such as wearable devices and biomedical or healthcare devices.

\subsection{Aims and structure of the thesis}

The main focus of this thesis is to explore the possibility of conducting polymers for nano-optics, or more specifically for plasmonics. This is an interdisciplinary study that combines organic electronics and plasmonics and is expected to be fun, creative, and full of unknowns together with opportunities. The thesis structure is exhibited in Figure 
1.1. We chose poly(3,4-ethylenedioxythiophene), or PEDOT, as our model system of conducting polymers due to its popularity and high performance ${ }^{33}$.

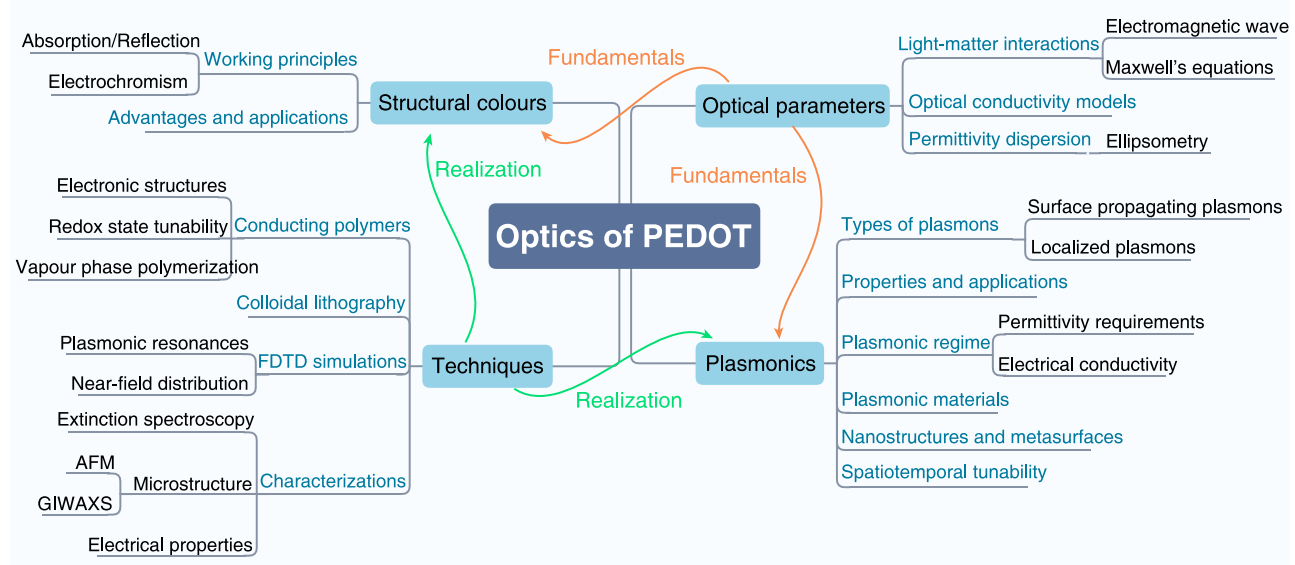

Figure 1.1 | The structure of this thesis. Optical parameters, plasmonics, structural colours, and techniques form four subtopics under the main topic "Optics of PEDOT".

The research addresses two main fundamental questions on the topic "Optics of PEDOT": the possibility to achieve plasmonic systems and redox-tunable structural colours with PEDOT. To answer these questions and to realize these materials or devices, we have to be equipped with basic knowledge of materials optics (Chapter 2), including the electromagnetic wave nature of light and how light interacts with matter. This information is stored in the optical parameters of the materials, and via suitable optical models, we can retrieve these parameters and evaluate the materials for different applications. Optical parameters can be measured via various methods, where ellipsometry represents one of the most accurate ones.

The main goal of this thesis was to demonstrate that conducting polymers can be used for plasmonics. We thus need to know the basics of plasmonics (Chapter 3 ) including how plasmons are generated or excited, what properties they have, and what applications they can be used for. We need to discuss advantages and disadvantages of current plasmonic materials. The absence of spatiotemporal tunability in traditional plasmonic systems motivates us to search for the next-generation plasmonic materials, and we thus have to know the basic criteria for plasmonic material.

This thesis proposes that conducting polymers (Chapter 4) can be one of such new plasmonic materials and we need to confirm this by showing evidence of plasmonic properties. Chapter 4 starts by introducing the basics of conducting polymers, including their redox state tunability, electronic structure, charge transport behaviour, and optical models. Such models are essential for determining the optical parameters of conducting polymers and thereby to evaluate their promise for plasmonics. In addition, the electrochromic properties of conducting polymers offers the possibility of altering their optical properties after fabrication. In this thesis, we combined this func- 
tion with thin film structural coloration, which enabled us to produce structural colours with redox state tunability.

Plasmons, especially localized plasmons, rely on nanostructures for applications in the visible and near-infrared spectral ranges. Thus, making nanostructures based on conducting polymers is a key element to this study (Chapter 5). To avoid unnecessary fabrication attempts, we designed suitable nanostructures before fabrication using the finite-difference time-domain method, which via numerical simulations predicts the nano-optical behaviour of different nanostructures. This approach enabled us to filter out suitable geometric parameters for fabrication. Finally, various characterization techniques helped us better understand our materials (e.g., vertical layer stratification in films) and to identify plasmonic resonances and evaluate their performance.

The last part of the thesis (Chapter 6) summarizes the results of the appended papers. We first proposed an optical model and a precise characterization methodology to extract the optical parameters of conducting polymers (Paper 1). Paper 2 focuses on the structural properties of conducting polymer thin films deposited via vapour phase polymerization. The study revealed a vertical inhomogeneity across the films, and we discussed the origin of this finding. In Paper 3 , we produced highly conducting polymer thin films and demonstrated that they could be used for a new type of plasmonics with redox tunability. Finally, Paper 4 presents a low-cost and facile method to produce tunable structural colour images based on UV-patterned conducting polymer thin films on metal surfaces. We think the results of this thesis have answered the two main questions that we have been trying to address, and hope that more progress can be achieved in the near future making this new research direction fruitful and prosperous.

\subsection{References}

$1 \quad$ Kartalopoulos, S. V. Introduction to DWDM technology: data in a rainbow. (SPIE Optical Engineering Press, 2000).

2 Liz-Marzán, L. Colloidal synthesis of plasmonic nanometals. (Jenny Stanford Publishing, 2020).

3 Maier, S. A. Plasmonics: fundamentals and applications. (Springer Science \& Business Media, 2007).

4 Murray, W. A. \& Barnes, W. L. Plasmonic materials. Advanced Materials 19, 37713782 (2007).

5 Mejía-Salazar, J. R. \& Oliveira Jr, O. N. Plasmonic biosensing: focus review. Chemical Reviews 118, 10617-10625 (2018).

6 Aslam, U., Chavez, S. \& Linic, S. Controlling energy flow in multimetallic nanostructures for plasmonic catalysis. Nature Nanotechnology 12, 1000 (2017).

7 Curto, A. G. et al. Unidirectional emission of a quantum dot coupled to a nanoantenna. Science 329, 930-933 (2010).

8 Xiao, M. et al. Plasmon-enhanced chemical reactions. Journal of Materials Chemistry A 1, 5790-5805 (2013).

9 Zhang, H. C. et al. A plasmonic route for the integrated wireless communication of subdiffraction-limited signals. Light: Science \& Applications 9, 1-9 (2020).

10 Garnett, E. C. et al. Self-limited plasmonic welding of silver nanowire junctions. Nature Materials 11, 241-249 (2012). 
11 Warren, S. C. \& Thimsen, E. Plasmonic solar water splitting. Energy \& Environmental Science 5, 5133-5146 (2012).

12 Shaltout, A. M., Shalaev, V. M. \& Brongersma, M. L. Spatiotemporal light control with active metasurfaces. Science 364 (2019).

13 Lu, W., Jiang, N. \& Wang, J. Active electrochemical plasmonic switching on polyaniline-coated gold nanocrystals. Advanced Materials 29, 1604862 (2017).

Duan, X., Kamin, S. \& Liu, N. Dynamic plasmonic colour display. Nature Communications 8, 1-9 (2017).

Fang, Z. et al. Gated tunability and hybridization of localized plasmons in nanostructured graphene. ACS Nano 7, 2388-2395 (2013).

Shirakawa, H., Louis, E. J., MacDiarmid, A. G., Chiang, C. K. \& Heeger, A. J. Synthesis of electrically conducting organic polymers: halogen derivatives of polyacetylene, $(\mathrm{CH})_{\mathrm{x}}$. Journal of the Chemical Society, Chemical Communications, 578-580 (1977).

Chiang, C. K. et al. Electrical conductivity in doped polyacetylene. Physical Review Letters 39, 1098 (1977).

Heeger, A. J. Semiconducting and metallic polymers: the fourth generation of polymeric materials (Nobel lecture). Angewandte Chemie International Edition 40, 2591-2611 (2001).

Someya, T., Bao,
379-385 (2016).

$20 \mathrm{Kim}, \mathrm{N}$. et al. in Conjugated Polymers: Properties, Processing, and Applications (CRC Press Taylor \& Francis Group, 2019).

21 Fabiano, S. et al. Poly (ethylene imine) impurities induce n-doping reaction in organic (semi) conductors. Advanced Materials 26, 6000-6006 (2014).

Bubnova, O., Berggren, M. \& Crispin, X. Tuning the thermoelectric properties of conducting polymers in an electrochemical transistor. Journal of the American Chemical Society 134, 16456-16459 (2012).

Rivnay, J. et al. Organic electrochemical transistors. Nature Reviews Materials 3, 1-14 (2018).

Friedlein, J. T., Donahue, M. J., Shaheen, S. E., Malliaras, G. G. \& McLeod, R. R. Microsecond response in organic electrochemical transistors: exceeding the ionic speed limit. Advanced Materials 28, 8398-8404 (2016).

Naik, G. V., Shalaev, V. M. \& Boltasseva, A. Alternative plasmonic materials: beyond gold and silver. Advanced Materials 25, 3264-3294 (2013).

Kim, N. et al. Role of interchain coupling in the metallic state of conducting polymers. Physical Review Letters 109, 106405 (2012).

Chen, S. et al. On the anomalous optical conductivity dispersion of electrically conducting polymers: ultra-wide spectral range ellipsometry combined with a DrudeLorentz model. Journal of Materials Chemistry C 7, 4350-4362 (2019).

Accounts of Chemical Research 18, 309-315 (1985).

Argun, A. A. et al. Multicolored electrochromism in polymers: structures and devices. Chemistry of Materials 16, 4401-4412 (2004).

1 Andersson, P., Forchheimer, R., Tehrani, P. \& Berggren, M. Printable all-organic electrochromic active-matrix displays. Advanced Functional Materials 17, 3074-3082 (2007).

Shanker, R. et al. Noniridescent Biomimetic Photonic Microdomes by Inkjet Printing. Nano Letters 20, 7243-7250 (2020).

3 Elschner, A., Kirchmeyer, S., Lovenich, W., Merker, U. \& Reuter, K. PEDOT: principles and applications of an intrinsically conductive polymer. (CRC press, 2010). 


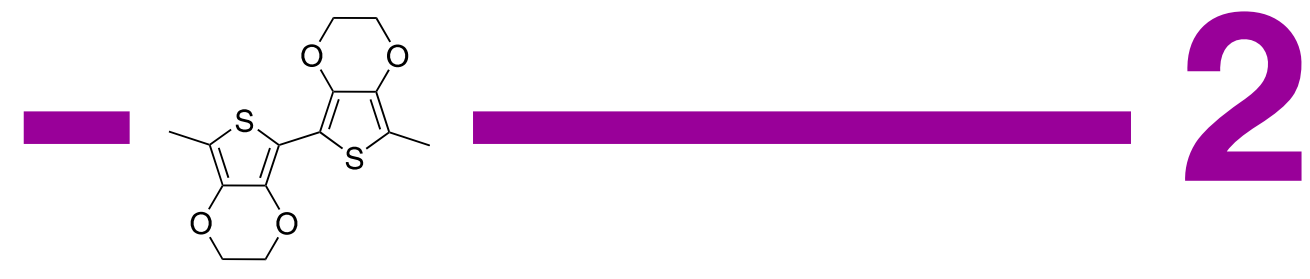

\section{Materials Optics}

The word "optics" comes from Greek with its original meaning "appearance", which was later developed into the study of light and light-matter interactions. In this chapter, we will briefly review the properties of light with special attention paid to the principles of electromagnetic wave propagation in vacuum and matter. At the end of the chapter, we will discuss optical models developed for different materials.

\section{Contents}

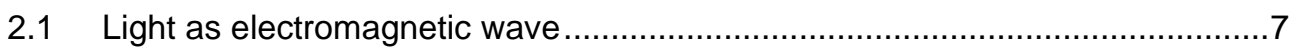

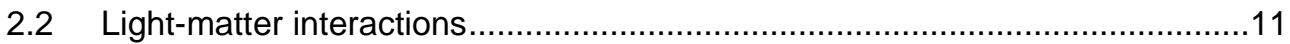

2.3 Optical models for materials ............................................................... 15

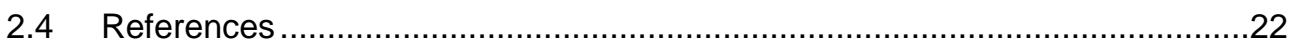

\subsection{Light as electromagnetic wave}

The earliest study on light could be traced back to ancient Greece, although optical components including lenses had already been utilized for years by ancient Egyptians and Mesopotamians ${ }^{1}$. The discussion on the nature of light had always been the focus of scientists and philosophers. The debate between the corpuscular (or particle) theory proposed by Rene Descartes and developed by Sir Isaac Newton and wave theory by Christiaan Huygens lasted for hundreds of years and was finally settled down by great 
efforts of James Clerk Maxwell, Max Planck and Albert Einstein². Light is now believed to possess a wave-particle duality, and we use either the wave properties or particle properties based on the particular phenomena or applications we are discussing. In this thesis, we treat light as an electromagnetic wave unless otherwise noted.

\subsubsection{Basic properties of electromagnetic waves}

Light propagating along z-axis (Figure 2.1) at time $t$ in vacuum can be described as a plane wave: ${ }^{3}$

$$
\begin{aligned}
& \mathbf{E}(\mathbf{r})=E_{0} \exp \left[i\left(\omega t-\mathbf{k} \cdot \mathbf{r}+\varphi_{0}\right)\right], \\
& \mathbf{B}(\mathbf{r})=B_{0} \exp \left[i\left(\omega t-\mathbf{k} \cdot \mathbf{r}+\varphi_{0}\right)\right],
\end{aligned}
$$

where $\mathbf{E}$ and $\mathbf{B}$ are the electrical and magnetic fields at position $\mathbf{r}, \mathrm{E}_{0}$ and $\mathrm{B}_{0}$ are the amplitudes (maximum) of the fields, $\omega$ is the angular frequency, $\mathbf{k}$ is the wavevector, and $\varphi_{0}$ is the initial phase constant. The wavevector denotes the propagating direction of the wave and its value is defined by $2 \pi / \lambda$, where $\lambda$ is the wavelength. The electromagnetic wave travels at a speed $\mathrm{c}$ of $3 \times 10^{8} \mathrm{~m} / \mathrm{s}$. The phase of this electromagnetic wave is represented by $\omega t-\mathbf{k} \cdot \mathbf{r}+\varphi_{0}$. Since we are dealing with non-magnetic materials, we only consider electrical properties of light in this thesis.

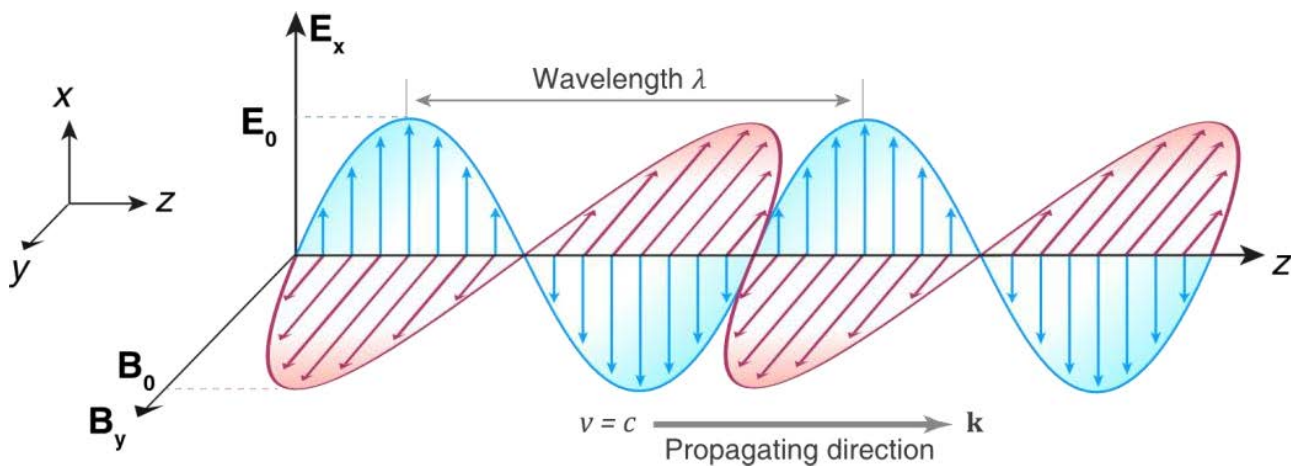

Figure 2.1 | An electromagnetic wave propagating along the z-axis. The electrical field $E_{x}$ ( $\mathrm{x}$-axis) and magnetic field $\mathrm{B}_{\mathrm{y}}(\mathrm{y}$-axis) are perpendicular to the propagation direction. The speed $\mathrm{c}$, wavelength $\lambda$, and wavevector $\mathbf{k}$ of the wave are indicated in the figure $\left(\mathrm{k}_{\mathrm{x}}=0\right.$ and $\left.\mathrm{k}_{\mathrm{y}}=0\right)$.

When multiple electromagnetic waves propagate along the same direction and their frequencies are identical, interferences between them can occur. The interference can be either constructive (increasing total amplitude of field intensities when their phases are identical) or destructive (decreasing total amplitude of field intensities when their phase difference is $\pi$ ).

Since both the electric and magnetic fields are perpendicular to the wavevector $\mathbf{k}$, light is a transverse wave. Polarization is one key character of light, describing the variation of field oscillation by time. The monochromatic plane wave shown in Figure 2.1 has a linear polarization of the electric field along the $x$-axis. The cases for combined waves 
can be complicated: when two orthogonal waves are in phase (phase shift of 0 or $2 \pi$ ) or out-of-phase (phase shift of $\pi$ ), it results in linearly polarized wave (Figure 2.2); when two waves have the same amplitude but a phase shift of $\pm 1 / 2 \pi$, it produces lefthand or right-hand circularly polarized light (Figure 2.2); and other situations will generate elliptically polarized light (Figure 2.2) or unpolarized light. More details on this can be found in Section 5.3.

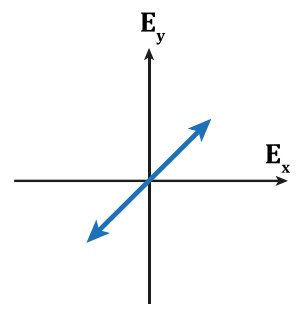

Linear polarization

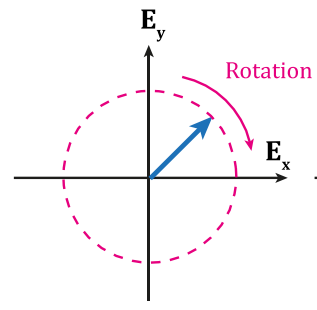

Left-hand circular polarization

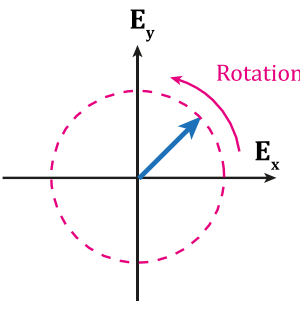

Right-hand circular polarization

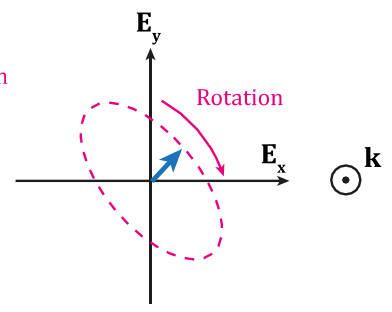

Elliptical polarization

Figure 2.2 | Polarization of light. The wavevector is perpendicular to the plane of the paper and pointing out of the page. Linearly polarized light can be formed by a combination of two orthogonal waves in phase (of same frequency). Circularly polarized light can be formed by two waves with identical amplitude and a phase difference of $\pm 1 / 2 \pi$. It can be classified as lefthanded or right-handed based on its rotation direction with respect to the propagation direction. Other cases (amplitude and phase differences) lead to elliptically polarized light.

The energy $E$ of the electromagnetic wave can be calculated via the Poynting vector, but a simple way would be considering the particle nature of light, photon: ${ }^{4}$

$$
\begin{gathered}
E=h v=h c / \lambda, \\
\mathbf{p}=\hbar \mathbf{k},
\end{gathered}
$$

where $h$ is the Planck constant $\left(6.63 \times 10^{-34} \mathrm{~m}^{2} \cdot \mathrm{kg} / \mathrm{s}\right), \hbar$ is the reduced Planck constant $(\hbar=h / 2 \pi), v$ is the frequency $(\nu=\omega / 2 \pi)$ and $\mathbf{p}$ is the momentum.

In optical measurements, it is impossible for us to measure the exact electric fields of the wave since their oscillations have an ultrahigh frequency especially for the visible range. Therefore, we normally measure the energy flux of the wave, which is defined as the rate of transfer of energy through a surface perpendicular to the wavevector ${ }^{5}$. This quantity is the "intensity" or "irradiance" $(I)$ in optical spectroscopy techniques, and is proportional to the square of the electric field amplitude: ${ }^{5}$

$$
I \propto\left|E_{0}\right|^{2} .
$$

Electromagnetic waves that exist in nature span more than 15 orders of magnitude in the frequency or wavelength scale (Figure 2.3), while light that human eye is sensitive to only covers a very narrow range from 400 to $700 \mathrm{~nm}$ (visible light). Electromagnetic waves from $\mathrm{THz}$ to UV are defined as the optical frequency range, which we frequently used for optical characterizations in the study presented in this thesis (e.g., spectroscopic ellipsometry6). 


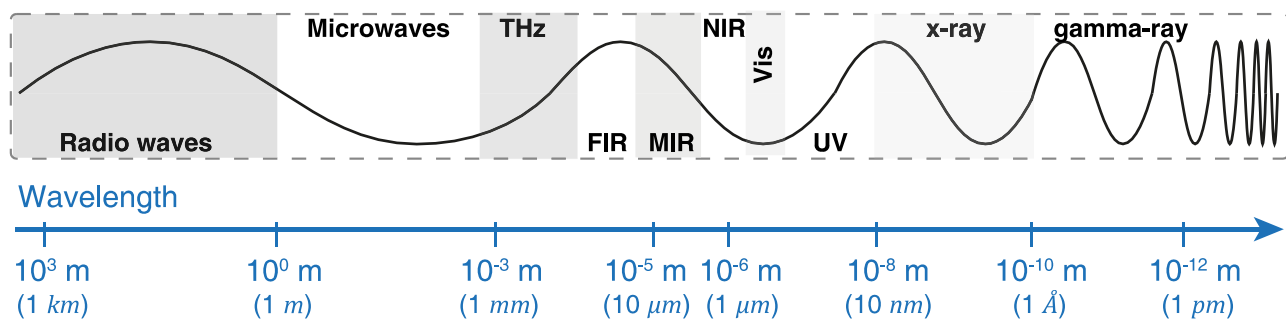

Frequency

$\begin{array}{lllll}1 \mathrm{MHz} & 1 \mathrm{GHz} & 1 \mathrm{THz} & 10^{3} \mathrm{THz} & 10^{6} \mathrm{THz}\end{array}$

Energy

$1 \mathrm{meV} \quad 1 \mathrm{eV} \quad 0.1 \mathrm{keV} \quad 10 \mathrm{keV}$

Figure 2.3 | Electromagnetic radiation spectrum. The electromagnetic waves are classified into different categories, from radio wave to gamma-ray, based on their wavelengths (blue axis), frequencies (green axis), and energies (magenta axis).

\subsubsection{Maxwell's equations}

Maxwell's equations are the fundamental principles of classical electromagnetism, explaining the creation and interrelation of electric and magnetic fields, summarized by James Clerk Maxwell. Through these equations, Maxwell predicted the existence of electromagnetic waves which were verified by Heinrich Hertz only after a few years ${ }^{2}$. The macroscopic formulation of the Maxwell's equations can be written in integral or differential form and the four equations in differential form express as 7,8

$$
\begin{gathered}
\nabla \cdot \mathbf{D}=\rho, \\
\nabla \cdot \mathbf{B}=0, \\
\nabla \times \mathbf{E}=-\partial \mathbf{B} / \partial t, \\
\nabla \times \mathbf{H}=j+\partial \mathbf{D} / \partial t,
\end{gathered}
$$

where $\mathbf{E}, \mathbf{D}, \mathbf{H}$, and $\mathbf{B}$ are the electric field, the electric displacement field, the magnetic field strength and the magnetic flux density, respectively. $\rho$ and $j$ are the charge and the current density. The four quantities for fields can be linked via the polarization $\mathbf{P}$ and magnetization $\mathbf{M}$ by 7,8

$$
\begin{gathered}
\mathbf{D}=\varepsilon_{0} \mathbf{E}+\mathbf{P}, \\
\mathbf{B}=\mu_{0} \mathbf{H}+\mathbf{M},
\end{gathered}
$$

where $\varepsilon_{0}$ is the vacuum permittivity $\left(8.85 \times 10^{-12} \mathrm{~F} / \mathrm{m}\right)$ and $\mu_{0}$ is the vacuum permeability. If we consider a linear, isotropic, and homogeneous material, Equation (2.5) can be simplified as 7,8

$$
\begin{aligned}
& \mathbf{D}=\varepsilon_{0} \varepsilon \mathbf{E}, \\
& \mathbf{B}=\mu_{0} \mu \mathbf{H},
\end{aligned}
$$


where $\varepsilon$ and $\mu$ are defined as the relative permittivity (or dielectric function) and relative permeability of the material. Equation (2.6) is known as constitutive relations.

The electromagnetic wave equations can be directly derived from Maxwell's equations by combining Equation (2.4c), (2.4d), and (2.6), which gives ${ }^{7,8}$

$$
\nabla \times \nabla \times \mathbf{E}=-\mu_{0} \frac{\partial^{2} \mathbf{D}}{\partial t^{2}}
$$

For homogeneous and isotropic materials, simplification of Equation (2.7) forms the electromagnetic wave equation $n^{7,8}$

$$
\nabla^{2} \mathbf{E}-\frac{\varepsilon}{c^{2}} \frac{\partial^{2} \mathbf{E}}{\partial t^{2}}=0
$$

where $c$ is the speed of electromagnetic wave $\left(c^{2}=1 / \varepsilon_{0} \mu_{0}\right)$. Assuming the electric field has a harmonic time dependence

$$
\mathbf{E}(\boldsymbol{r}, t)=\mathbf{E}(\mathbf{r}) e^{-i \omega t},
$$

the Equation (2.8) can be further simplified to ${ }^{7,8}$

$$
\nabla^{2} \mathbf{E}+k_{0}^{2} \varepsilon \mathbf{E}=0,
$$

where $k_{0}$ is the wavenumber for the wave in vacuum $\left(k_{0}=\omega / c\right)$. This is known as the Helmholtz equation, describing the behaviour of electromagnetic waves in isotropic and homogeneous matters.

\subsection{Light-matter interactions}

Light-matter interactions represent the core of optics and nano-optics, where we are trying to understand and utilize different mechanisms. The wave nature of light gives the phenomena of transmission, reflection, refraction, and diffraction, and the particle nature of light produces the cases of absorption and scattering ${ }^{2}$ (Figure 2.4). In this section, we will focus on transmission, reflection, refraction, and absorption, since they are most relevant for this thesis. The scattering part will be introduced in Section 3.2.

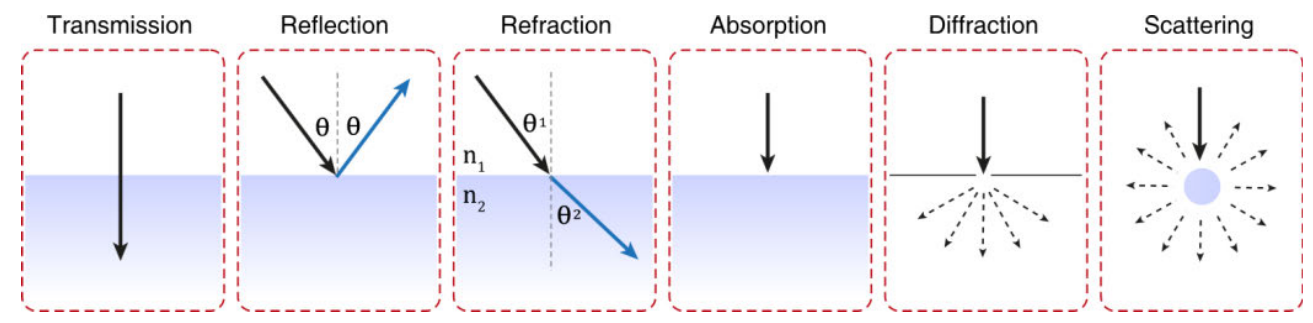

Figure 2.4 | Interaction mechanisms between light and matter. Typically, transmission, reflection, refraction, and diffraction are regarded as phenomena originating from the wave nature of light, while absorption and scattering are due to the particle nature of light.

\subsubsection{Transmission and Extinction}


When a light beam passes through a structure, we can measure the transmittance of the light (Figure 2.4) as the ratio of the outgoing and incident light intensity: ${ }^{5}$

$$
T=I_{\text {out }} / I_{\text {in }} \text {. }
$$

The intensity loss of the light is defined as the extinction of the structure:

$$
E=1-T .
$$

The extinction of light can be caused by various mechanisms, such as reflection or/and absorption. In spectroscopy techniques, the absorbance $A$ is defined as ${ }^{5}$

$$
A=\log _{10}\left(\frac{I_{\text {in }}}{I_{\text {out }}}\right)=-\log _{10} T,
$$

although this quantity is related to the extinction of light and not only absorption. Neglecting reflection and scattering, Beer-Lambert law defines the relationship between the absorbance and other material parameters: 5

$$
A=\alpha c l,
$$

where $\alpha$ is the molar absorption coefficient, $c$ is the molar concentration, and $l$ is the optical path length for a homogeneous solution.

\subsubsection{Reflection and refraction}

At the interface between two materials, light can be reflected back and this phenomenon is defined as reflection. Following the law of reflection, the incident light beam and reflected light beam propagate in the plane of incidence, which is defined by the wavevector of the incoming beam and the interface normal ${ }^{2}$ (Figure 2.4). The incident angle is equal to the reflected angle. The intensity ratio is defined as the reflectance $R$ of the structure: ${ }^{5}$

$$
R=I_{\text {reflect }} / I_{\text {in }},
$$

where $I_{\text {reflect }}$ is the reflected light intensity and $I_{\text {in }}$ is the incident light intensity.

The reflectance depends on the surface morphology, the angle of incidence, the polarization of light and the optical properties of the material involved ${ }^{5}$. Metal and metalcoated glass mirrors can have very smooth surfaces, which together with their material properties lead to high reflectance.

Refraction, on the other hand, describes the behaviour of a light beam transmitting from medium (1) to medium (2). It is governed by Snell's law: ${ }^{2}$

$$
n_{1} \sin \theta_{1}=n_{2} \sin \theta_{2},
$$

where $\theta_{1}$ and $\theta_{2}$ are the light propagating angles with respect to the interface normal in the two media and $n_{1}$ and $n_{2}$ are the refractive indices of the two media (Figure 2.4). The refractive index measures the phase velocity of light in the material and is given by the ratio of the light speed in vacuum $(c)$ to that in the medium $(v)$ :

$$
n=c / v \text {. }
$$

Accounting for incident angle and polarization, the reflectance at an interface can be described by the Fresnel equations: ${ }^{5}$ 


$$
\begin{aligned}
& R_{s}=\left|\frac{n_{1} \cos \theta_{1}-n_{2} \cos \theta_{2}}{n_{1} \cos \theta_{1}+n_{2} \cos \theta_{2}}\right|^{2}, \\
& R_{p}=\left|\frac{n_{1} \cos \theta_{2}-n_{2} \cos \theta_{1}}{n_{1} \cos \theta_{2}+n_{2} \cos \theta_{1}}\right|^{2},
\end{aligned}
$$

where $\theta_{1}$ and $\theta_{2}$ can be calculated by Snell's law in Equation (2.16). The subscripts, $s$ and $p$, indicate the polarization directions of the light (see Figure 2.5). The plane of incidence is formed by wavevector of the incoming beam and the interface normal. $s$ originates from the German word "senkrecht", meaning normal, and it is the direction perpendicular to the plane of incidence. $p$, with the meaning of "parallel", is within the plane of incidence. Both $s$ and $p$ directions are perpendicular to the light propagation direction. It should be highlighted that the refractive index used in the equations can be complex numbers (see details

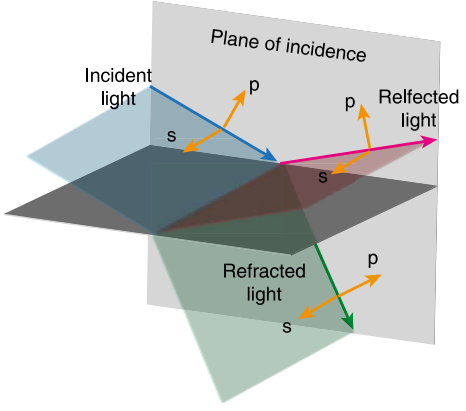

Figure $2.5 \mid s$-/p-polarization directions for incident light. in Section 2.3.2).

An important phenomenon can occur when the incident angle is ${ }^{6}$

$$
\theta_{\text {Brewster }}=\arctan \left(n_{2} / n_{1}\right) \text {. }
$$

At this specific angle, or Brewster angle, the reflected ray is completely polarized in $s$ direction since the reflectance for $p$-direction is zero (combining Equations (2.19) and (2.16), and (2.18)). This effect can be utilized for polarizers.

\subsubsection{Absorption}

Absorption of light in a material is mostly related to its electronic structures. The electrons in the atoms or molecules can make transitions between two energy levels when incident light carries the energy identical to the energy spacing. For organic materials that are discussed in this thesis, three main types of energy levels exist, namely electronic, vibrational, and rotational energy levels (Figure 2.6).

Organic materials are different from crystalline inorganic materials, where periodic lattice results in an energy band structure and the spacing between two nearby energy levels is negligibly small (and thermal energy $k_{B} T$ from the surrounding is large enough to initiate the transition). In conducting polymers, charge carriers generated by counterion doping can form polaronic or bipolaronic bands in the electronic structure (see Section 4.2). Additional absorption fingerprints of doping can be observed in doped conducting polymers due to these electronic transitions between (bi-)polaronic bands and conduction (valence) band ${ }^{10}$.

Electronic, vibrational, and rotational transitions can easily be identified from their energy scales and spectral ranges. UV-Vis-NIR spectroscopies are designed to probe electronic transitions, IR spectroscopies are useful for vibrational transitions (or 
phonons in inorganics), and rotational transitions can be measured by FIR or microwave spectro-scopies, due to their ultralow energies ${ }^{11}$.

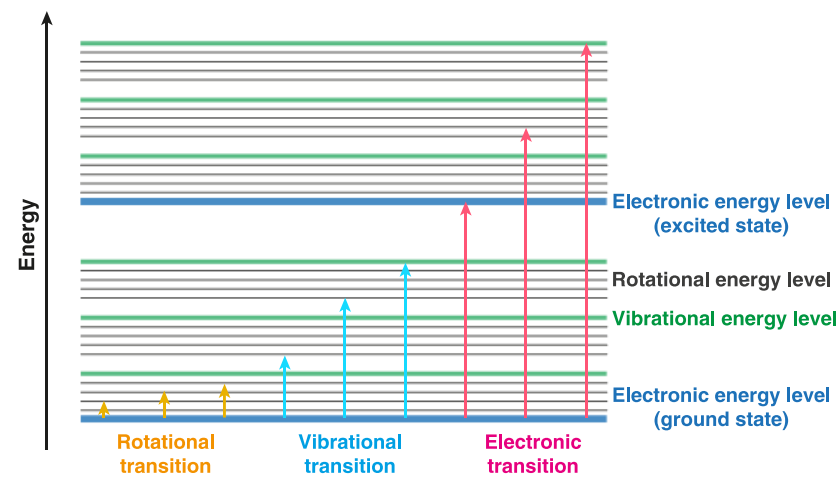

Figure 2.6 | Molecular energy levels and related transitions. Energy levels in materials can form energy bands (the top band is excited state and the bottom one is ground state). Electronic transitions are inter-band transitions while vibrational or rotational transitions are intra-band transitions with much smaller energies. Transition can occur when the energy is exactly the same as the energy difference of any two levels. The figure is modified from literature ${ }^{9}$.

Two important phenomena related to the absorption of light are the photovoltaic effect and the photoelectric effect. The photovoltaic effect is due to electronic transition, where electrons in the valence band transit to the conduction band by absorbing light with energy larger than the band gap ${ }^{12}$. Based on this effect, semiconducting polymers are used as active layers for photovoltaic cells, or as emissive layers in light-emitting diodes by the inverse process, photon emission ${ }^{13}$. The photoelectric effect requires higher energy to excite the electron from the ground state to the vacuum level, becoming free electrons. Typical example for this effect is X-ray and ultraviolet photoelectron spectro-scopies, where high-energy photons are used to excite valance and core electrons of the molecules or atoms. Band structure (ultraviolet photons) and elemental information (X-ray photons) of the material can thus be extracted by these characterizations techniques ${ }^{14}$. In Chapter 3 , we will discuss how plasmons can also contribute to absorption in different materials and nanostructures.

\subsection{Optical models for materials}

Based on differences in electrical properties, materials can be divided into three main classes: insulators, semiconductors, and conductors (Figure 2.7).

The fundamental difference between them lies in the electronic structure (or energy diagram) of the material (Figure 2.7). For insulators and semiconductors, a gap exists between the valence and the conduction bands ${ }^{15}$. To conduct electricity, free electrons (holes) are required in the conduction (valence) band of the material. For both insulator 
and semiconductor, an external energy is needed to excite electrons (holes) from the completely occupied valence (conduction) band to the completely empty conduction (valence) band. Due to the relatively small band gap in semiconductors (smaller than $3 \mathrm{eV}$ ), external heating or radiation is sufficient for such electronic transition and thus semiconductors are known as thermosensitive or photo-sensitive. We start this section with discussing the electrical conductivity of materials.

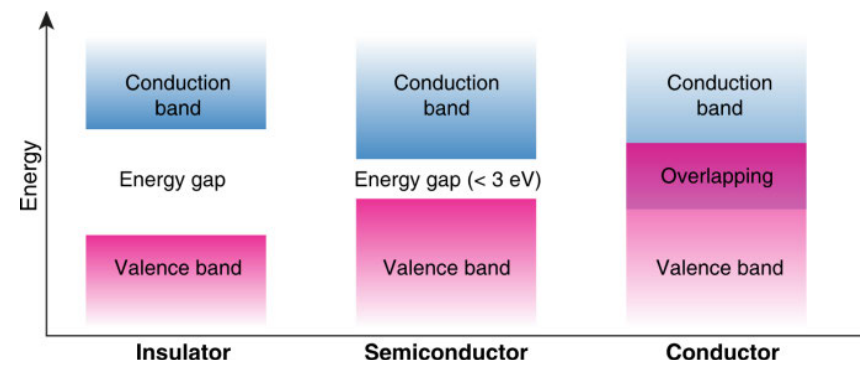

Figure 2.7 | Energy diagram for different classes of materials. Conductors have no band gap, semiconductors have band gaps smaller than $3 \mathrm{eV}$, and insulators have larger band gaps.

\subsubsection{Electrical conductivity of materials}

The electrical conductance $G$ describes the ease of an electric current to pass through a device or system, and can be calculated by the ratio of measured electrical current $I$ and applied voltage bias $V$, or reciprocal of the electrical resistance $R:^{16}$

$$
G=I / V=1 / R \text {. }
$$

The conductance strongly depends on the geometric factors of the device, and a more fundamental materials parameter is the electrical conductivity $\sigma$, which is defined via ${ }^{16}$

$$
\sigma=\frac{1}{\rho}=G \frac{L}{A} .
$$

where $\rho$ is the electrical resistivity, $L$ is the length (parallel to the current direction) and $A$ is the cross-sectional area (normal to the current direction) of the device. Therefore, Ohm's Law in Equation (2.20) can be written in a new form: ${ }^{16}$

$$
j=\sigma|\mathbf{E}|,
$$

where $j$ is the current density $(j=I / A)$ and $\mathbf{E}$ is the electric field in the device $(|\mathbf{E}|=V / L)$. This equation describes the microscopic and local electrical properties of the material. For materials with electrons as charge carriers (e.g., metals), the electrical conductivity is determined by ${ }^{16}$

$$
\sigma=n e \mu \text {. }
$$

where $n$ is the charge carrier (electron) concentration, $e$ is the elemental charge (1.6 $\times 10^{-19} \mathrm{C}$ ), and $\mu$ is the carrier mobility. Carrier mobility describes how easily carrier can move in the material within an electric field, and it can be influenced by various microstructural factors (e.g., crystallinity, morphology, and traps ${ }^{17}$ ). The average drift velocity of a charge carrier can be calculated by ${ }^{16}$ 


$$
v_{\text {drift }}=\mu|\mathbf{E}| .
$$

The motion of electrons in materials with periodic lattice structures (e.g., metals or semiconductors) is not ballistic and collisions occur frequently between atomic nuclei and electrons and between electrons. These collisions result in scattering of electrons and thus electric field induced speed acceleration of electrons, which balances the electric field-induced acceleration of electrons and leads to saturation of the speed, resulting in an average drift velocity of diffusive transport above.

Another way to form an electrical current is by diffusion using the concentration gradient of electrons as driving force: ${ }^{16}$

$$
j_{\text {diffusion }}=e D \frac{d n}{d x},
$$

where $D$ is the diffusion constant of the carriers in the material. The diffusion constant is associated with the carrier mobility via the Einstein relation ${ }^{16}$ :

$$
D=\mu k_{B} T / e,
$$

where $k_{B}$ is the Boltzmann constant and $T$ is the temperature in kelvin. Therefore, the total current density in a metal (electron as the charge carrier) is represented by

$$
j_{\text {total }}=j_{\text {drift }}+j_{\text {diffusion }}=n e \mu|\mathbf{E}|+e D \frac{d n}{d x} .
$$

\subsubsection{Optical parameters}

When the electrical current in a material is alternating $(A C)$ instead of being static or direct (DC) as in Section 2.3.1, the frequency dependence has to be considered. Equation (2.22) can be written in a frequency-dependent form:

$$
j(\omega)=\sigma(\omega)|\mathbf{E}(\omega)| .
$$

The electrical current in the material can be described by its electric field polarization density $P$ (see Equation (2.5) and this quantity should not be confused with light polarization): 8

$$
j=\frac{\partial P}{\partial t} .
$$

This equation can be written in the Fourier domain $a^{8}$

$$
j(\omega)=-i \omega P(\omega),
$$

where $i$ is the imaginary unit. Combining Equations (2.5a), (2.6a), (2.28), and (2.30), we obtain ${ }^{8}$

$$
\sigma(\omega)=\sigma_{1}(\omega)+i \sigma_{2}(\omega)=-i \omega \varepsilon_{0}(\varepsilon(\omega)-1)
$$

or

$$
\varepsilon(\omega)=\varepsilon_{1}(\omega)+i \varepsilon_{2}(\omega)=1+\frac{i \sigma(\omega)}{\omega \varepsilon_{0}},
$$

where we define $\sigma(\omega)$ as the optical conductivity (where at low frequencies could also be named $\mathrm{AC}$ conductivity) and $\varepsilon(\omega)$ the dielectric function or permittivity. Both quantities are complex variables and subscript 1 indicates the real part while 2 stands for the imaginary part. It is highlighted that the two quantities are no longer constant as in 
Equation (2.6) and they have frequency dispersions. Both optical conductivity and permittivity describe changes between stimulus (e.g., electric field) and response (e.g., electrical current) not only in amplitude but also in phase.

We call the two quantities as the optical parameters of the material. They contain a variety of useful information about the material, including different physical processes from charge carrier transport, inter-band transitions and phonon vibrations, to localized charge carrier absorption at low frequencies ${ }^{18}$. The permittivity or optical conductivity below the optical frequency range (see Section 2.1.1) is also valuable for investigation of dielectric or ionic behaviour of materials ${ }^{19,20}$. In this thesis we only focus on optical conductivity and permittivity in the optical frequency range. A schematic of typical processes in the permittivity dispersions is shown in Figure 2.8.
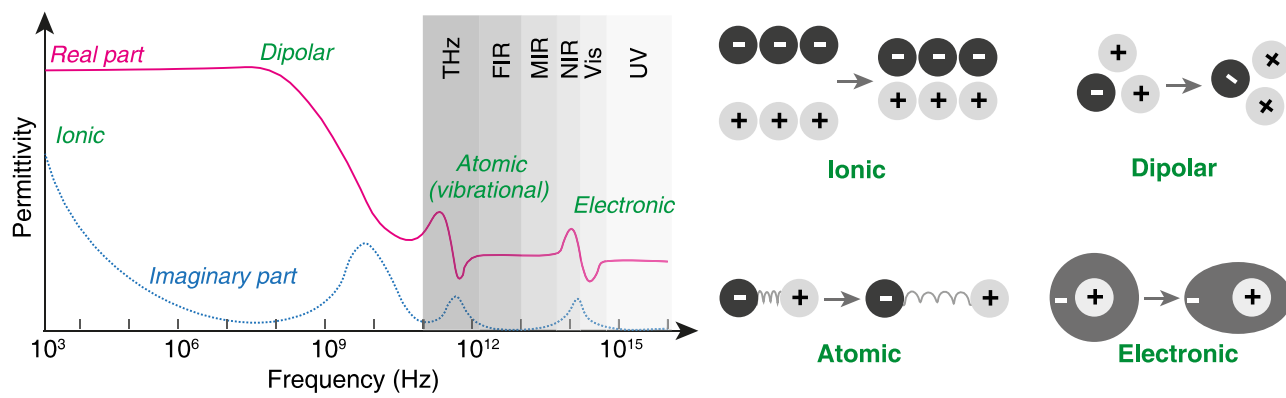

Figure 2.8 | Typical physical processes identifiable from the permittivity dispersions. The left part schematically shows the permittivity dispersion from microwave to UV ranges. Different physical processes can be identified based on their energy scales. The right part are four typical processes that can occur in organic materials. The figure is modified from literature ${ }^{21,22}$.

Optical parameters can be measured through various methods, including reflectivity studies. In a standard reflectivity measurement, we can obtain another type of optical parameters, the complex refractive index which is defined through the permittivity as ${ }^{17}$

$$
\tilde{n}(\omega)=\sqrt{\varepsilon(\omega)}=n(\omega)+i k(\omega) .
$$

The real part of it, $n$, is the refractive index used in Equation (2.17) and its imaginary part, $k$, is the extinction coefficient. More specifically, $n$ and $k$ can be obtained by 4,17

$$
\begin{aligned}
& n(\omega)=\sqrt{\frac{1}{2}\left(\sqrt{\varepsilon_{1}(\omega)^{2}+\varepsilon_{2}(\omega)^{2}}+\varepsilon_{1}(\omega)\right)}, \\
& k(\omega)=\sqrt{\frac{1}{2}\left(\sqrt{\varepsilon_{1}(\omega)^{2}+\varepsilon_{2}(\omega)^{2}}-\varepsilon_{1}(\omega)\right)} .
\end{aligned}
$$

The extinction coefficient is related to the molar absorption coefficient (see Equation (2.14)) of the material by ${ }^{4}$

$$
\alpha(\omega)=\frac{2 k(\omega) \omega}{c}=\frac{4 \pi k(\omega)}{\lambda} .
$$

The three categories of optical parameters (i.e., optical conductivity, permittivity, and complex refractive index) contain the same information and are interrelated via Equa- 
tions (2.31), (2.32), and (2.33). The permittivity and complex refractive index are preferred for optical studies.

The real part and imaginary part of the optical parameters are not independent, and the relation follows the Kramers-Kronig relations. For the case of permittivity, we have ${ }^{4}$

$$
\begin{aligned}
& \varepsilon_{2}(\omega)=-\frac{2 \omega}{\pi} P \int_{0}^{\infty} \frac{\varepsilon_{1}\left(\omega^{\prime}\right)-1}{\omega^{\prime 2}-\omega^{2}} d \omega^{\prime}, \\
& \varepsilon_{1}(\omega)-1=\frac{2}{\pi} P \int_{0}^{\infty} \frac{\varepsilon_{2}\left(\omega^{\prime}\right) \omega^{\prime}}{\omega^{\prime 2}-\omega^{2}} d \omega^{\prime},
\end{aligned}
$$

where $P$ denotes the principal value of the integrals. Standard reflectivity measurement to determine optical parameters can provide only one part of the complex refractive index, and the other part can be derived with this Kramers-Kronig transformation ${ }^{23-25}$. Since the integral is from zero to infinity, hence ranging beyond measurement limits, several assumptions have to be made when using this method, making it less accurate. Different from reflectivity measurements, ellipsometry uses polarized light and multiple incident angles, collecting more information (amplitude and phase) and no hypothesis on material properties beyond the measurement range is needed (see Section 5.3). Ellipsometry is therefore considered as the most reliable and accurate approach in determining optical parameters ${ }^{5}$.

In the above discussion, we assumed that the material is isotropic, which indicates no preferential direction for light transport and the optical parameter is a scalar. However, many materials are anisotropic, especially polymeric materials deposited via solutionbased processes ${ }^{26-30}$. The optical parameter thus has to be a tensor:

$$
\varepsilon(\omega)=\left[\begin{array}{lll}
\varepsilon_{x x} & \varepsilon_{x y} & \varepsilon_{x z} \\
\varepsilon_{y x} & \varepsilon_{y y} & \varepsilon_{y z} \\
\varepsilon_{z x} & \varepsilon_{z y} & \varepsilon_{z z}
\end{array}\right] .
$$

This permittivity tensor can be simplified if the crystal axes coincide with the axes of the coordinate system and if there is no birefringence or circular dichroism in the material: 4,26

$$
\varepsilon(\omega)=\left[\begin{array}{ccc}
\varepsilon_{x} & 0 & 0 \\
0 & \varepsilon_{y} & 0 \\
0 & 0 & \varepsilon_{z}
\end{array}\right] .
$$

The material is thus defined as a biaxially anisotropic material. If, for example, the permittivity for $x$-axis and $y$-axis are identical, then the material is a uniaxially anisotropic material. For our polymeric films, we define the $z$-axis as the out-of-plane direction and any direction inside the plane formed by the $x$-axis and the $y$-axis as the in-plane direction ${ }^{28}$. An advantage of spectroscopic ellipsometry over standard reflectivity measurements is that it can provide information on material anisotropy ${ }^{28,31}$.

The optical parameters contain rich information of the material, but extracting them from ellipsometric raw data requires an optical model of the material stack involved. In the following section, we start by considering the case of metal and then derive the Drude model for it. The derivation is based on several approximations ${ }^{32}$ and the real case can be much more complicated and is beyond the scope of the thesis. 


\subsubsection{Electron transport in metal described by the Drude model}

For an electron under the influence of an electric field of $\mathbf{E}$, the equation of motion is

$$
m \frac{d^{2} \mathbf{x}}{d t^{2}}+m \gamma \frac{d \mathbf{x}}{d t}=-e \mathbf{E},
$$

where $\gamma$ describes the characteristic frequency of electron collisions during its motion inside the metal and the reciprocal quantity $\tau$ is the average time between two consecutive collisions of the electron $(\gamma=1 / \tau)$. Assuming that the field from an electromagnetic wave has a harmonic time dependence as in Equation (2.9), we then have

$$
\mathbf{x}(t)=\frac{e}{m\left(\omega^{2}+i \omega \gamma\right)} \mathbf{E}(t) .
$$

The polarization of the metal could be understood as the electric dipole formed by the displacement of all free (conduction band) electrons, and therefore the electric field polarization (see Equation (2.5)) can be expressed as ${ }^{8}$

$$
\mathbf{P}(t)=-n e \mathbf{x}(t)=-\frac{n e^{2}}{m\left(\omega^{2}+i \omega \gamma\right)} \mathbf{E}(t) .
$$

Using Equation (2.5a), we have ${ }^{8}$

$$
\mathbf{D}(t)=\varepsilon_{0}\left(1-\frac{n e^{2}}{m \varepsilon_{0}\left(\omega^{2}+i \omega \gamma\right)}\right) \mathbf{E}(t) .
$$

If the material conforms the constitute relation of Equation (2.6), we can obtain the formula for the permittivity of the metal, known as the Drude model:8,32

$$
\varepsilon(\omega)=1-\frac{n e^{2}}{m \varepsilon_{0}\left(\omega^{2}+i \omega \gamma\right)}=1-\frac{\omega_{p}^{2}}{\omega^{2}+i \omega \gamma},
$$

where $\omega_{p}$ is defined as the plasma frequency of the material: 8

$$
\omega_{p}=\sqrt{\frac{n e^{2}}{m \varepsilon_{0}}} .
$$

The plasma frequency $\omega_{p}$ is a characteristic parameter of a metal, indicating the approximate position at which the permittivity changes sign (from positive to negative values $)^{17}$. The real and imaginary parts of the Drude permittivity are

$$
\begin{aligned}
& \varepsilon_{1}(\omega)=1-\frac{\omega_{p}^{2} \tau^{2}}{1+\omega^{2} \tau^{2}}, \\
& \varepsilon_{2}(\omega)=\frac{\omega_{p}^{2} \tau}{\omega\left(1+\omega^{2} \tau^{2}\right)} .
\end{aligned}
$$

Based on Equation (2.31), the optical conductivity of the Drude model is

and its real and imaginary parts are

$$
\sigma(\omega)=\frac{\varepsilon_{0} \omega_{p}^{2} \tau}{1-i \omega \tau}
$$

$$
\begin{aligned}
& \sigma_{1}(\omega)=\frac{\varepsilon_{0} \omega_{p}^{2} \tau}{1+\omega^{2} \tau^{2}}, \\
& \sigma_{2}(\omega)=\frac{\omega \varepsilon_{0} \omega_{p}^{2} \tau^{2}}{1+\omega^{2} \tau^{2}} .
\end{aligned}
$$


Typical features of the Drude model and the influences of carrier mobility and density on its permittivity and optical conductivity are shown in Figure 2.9.

We can derive the DC conductivity of the metal from the real optical conductivity at angular frequency $\omega$ equal to zero:32,33

$$
\sigma_{D C}=\sigma_{1}(0)=\varepsilon_{0} \omega_{p}^{2} \tau=\frac{n e^{2} \tau}{m},
$$

which is the definition of electrical conductivity in Equation (2.23), and the mobility $\mu$ is represented by $e \tau / \mathrm{m}$. Therefore, the optical conductivity and the permittivity formula of the Drude model could also be expressed using carrier density (see Equation (2.44)) and mobility as parameters:

$$
\begin{gathered}
\sigma(\omega)=\frac{n e^{2}}{e / \mu-i m \omega}, \\
\varepsilon(\omega)=1-\frac{n e^{2}}{\varepsilon_{0}\left(m \omega^{2}+i \omega e / \mu\right)} .
\end{gathered}
$$
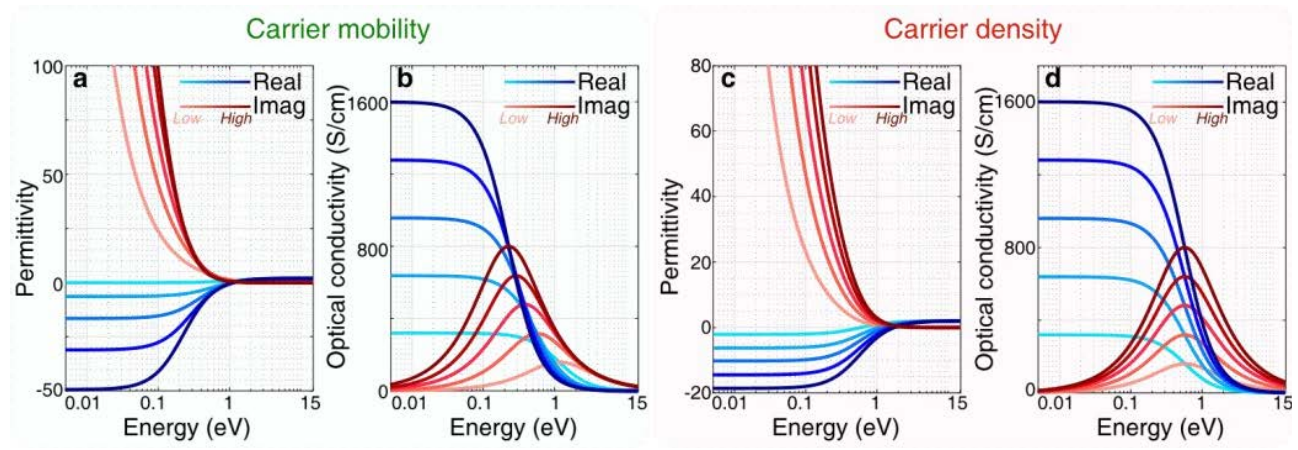

Figure 2.9 | Optical parameter dispersions based on the Drude model. a and $\mathbf{b}$, The effect of carrier mobility on the permittivity (a) and optical conductivity (b) for a fixed carrier density of $2 \times 10^{21} \mathrm{~cm}^{-3}$. The carrier mobility was varied from $1 \mathrm{~cm}^{2} / \mathrm{V} \cdot \mathrm{s}$ (lightest colour) to $5 \mathrm{~cm}^{2} / \mathrm{V} \cdot \mathrm{s}$ (darkest colour). c and $\mathbf{d}$, The effect of carrier density on the permittivity (c) and optical conductivity (d) for a fixed carrier mobility of $1 \mathrm{~cm}^{2} / \mathrm{V} \cdot \mathrm{s}$. The carrier density was varied from $1 \mathrm{x}$ $10^{21} \mathrm{~cm}^{-3}$ (lightest colour) to $5 \times 10^{21} \mathrm{~cm}^{-3}$ (darkest colour). In the graph, the real part is plotted in blue colour while the imaginary part is in red colour.

Tuning the two parameters ( $n$ and $\mu$ ), we can see changes in the overall shapes of the optical conductivity and permittivity (Figure 2.9). It is clear that an increment in either parameters increases the real part of the optical conductivity at low frequencies, while it lowers the real part of the permittivity towards negative values. For visible and infrared ranges, where the angular frequency is lower than the plasma frequency for common metals, the refractive index $n$ is close to zero and the extinction coefficient $k$ becomes dominant according to Equation (2.33). Based on Equation (2.18), the reflection at normal incidence for an air-metal interface can then be approximated as

$$
R \approx \frac{\left|1-i k_{\text {metal }}\right|^{2}}{\left|1+i k_{\text {metal }}\right|^{2}}=\frac{1+k_{\text {metal }}{ }^{2}}{1+k_{\text {metal }}{ }^{2}}=1 \text {. }
$$


Therefore, we normally utilize metals as mirrors due to this high reflectance property. In some cases, the imaginary part of the material permittivity is small compared to the real counterpart. Neglecting the imaginary part, we obtain the simplified Drude model:

$$
\varepsilon(\omega)=1-\frac{\omega_{p}^{2}}{\omega^{2}} .
$$

The real cases for metals are complicated, and additional modifications, such as terms accounting for interband transitions, are needed to be added to the Drude formula.

\subsubsection{Optical models for other materials}

For insulator with large bandgap (see Figure 2.7), the absorption in the visible and NIR is almost zero. Its optical parameters can be described by the Cauchy model (neglecting phonon or vibrational modes at lower frequencies). This is an empirical model for transparent materials, and its optical parameter are expressed as ${ }^{34}$

$$
\left\{\begin{array}{l}
n=A+\frac{B}{\lambda^{2}}+\frac{C}{\lambda^{4}}, \\
k=0
\end{array}\right.
$$

where $A, B$, and $C$ are material coefficients with no certain physical meanings. Cauchy model does not conform Kramers-Kronig consistency in Equation (2.36) ${ }^{4}$.

An absorption (Urbach) tail is sometimes added to the Cauchy model as the extinction coefficient part: 35,36

$$
k=\alpha e^{\beta(1.24 \mu m(1 / \lambda-1 / \gamma))},
$$

where $\alpha$ is the extinction coefficient amplitude, $\beta$ is the exponent factor, and $\gamma$ is the band edge (absorption threshold).
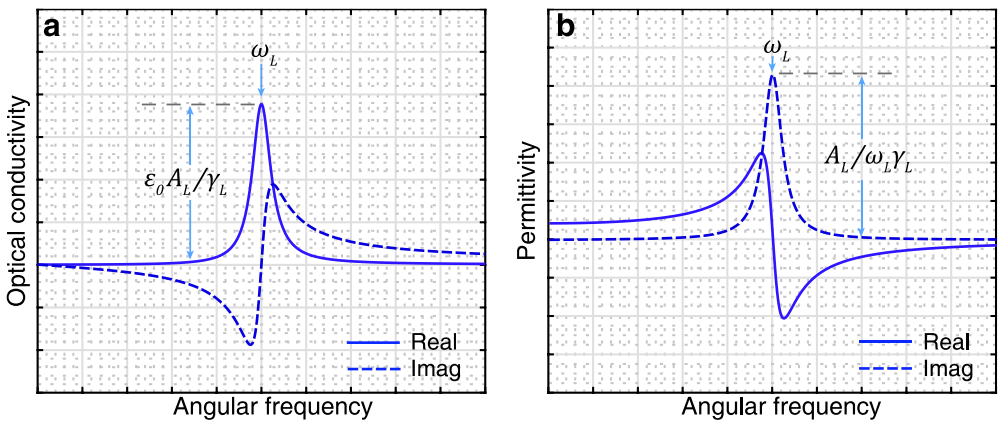

Figure 2.10 | Optical conductivity and permittivity of Lorentz oscillator model. a, Optical conductivity, and $\mathbf{b}$, permittivity dispersion curves. Solid lines are real parts and dashed lines are imaginary parts. The characteristic angular frequency $\omega_{L}$, amplitude $A_{L}$, and broadening $\gamma_{L}$ of the oscillator are indicated in the figure.

The Lorentz model, or Lorentz (harmonic) oscillator, is based on classical harmonic dipole oscillations, and therefore largely utilized to describe physical processes related 
to resonance polarizations in materials ${ }^{5}$. The optical conductivity and permittivity originating from a Lorentz oscillator can be expressed as ${ }^{17}$

$$
\begin{gathered}
\sigma_{L}(\omega)=\frac{i \varepsilon_{0} \omega A_{L}}{\omega^{2}-\omega_{L}^{2}+i \omega \gamma_{L}}, \\
\varepsilon_{L}(\omega)=-\frac{A_{L}}{\omega^{2}-\omega_{L}^{2}+i \omega \gamma_{L}} .
\end{gathered}
$$

where $A_{L}, \omega_{L}$, and $\gamma_{L}$ are amplitude, characteristic angular frequency, and broadening of the oscillator. If $\omega_{L}$ is set to zero, the formula returns to the Drude model in Equation (2.43) and (2.46). Figure 2.10 shows the overall shape of Lorentz oscillator, which can generate a peak in the real optical conductivity and form negative values in the real permittivity on the high energy (angular frequency) side of the resonance.

For semiconductors, there is a Tauc-Lorentz model with consideration of band gap ${ }^{37}$, and for composite materials, effective medium approximations accounting for different components are widely adopted ${ }^{4}$. However, both models are beyond the scope of the thesis which deals with polymeric conductors, and will not be introduced in details.

\subsection{References}

Enoch, J. M. Archeological optics: the very first known mirrors and lenses. Journal of Modern Optics 54, 1221-1239 (2007).

Hecht, E. Optics. (Addison-Wesley, 2002).

Kasap, S. O. Optoelectronics and photonics. (Prentice Hall, 2001).

Arwin, H. Thin Film Optics and Polarized Light. (Hans Arwin, 2015).

Tompkins, H. G. \& Hilfiker, J. N. Spectroscopic Ellipsometry: Practical Application to Thin Film Characterization. (Momentum Press, 2016).

\section{Tompkins, H. \& Irene, E. A. Handbook of ellipsometry. (William Andrew, 2005).}

Griffiths, D. J. Introduction to electrodynamics. (Pearson \& Cambridge University Press, 1999).

Maier, S. A. Plasmonics: fundamentals and applications. (Springer Science \& Business Media, 2007).

Wenzel, T. Molecular and atomic spectroscopy. (LibreTexts, 2020).

Zozoulenko, I. et al. Polarons, bipolarons, and absorption spectroscopy of PEDOT. ACS Applied Polymer Materials 1, 83-94 (2018).

Townes, C. H. \& Schawlow, A. L.Microwave spectroscopy. (Courier Corporation, 2013). Wenham, S. R. Applied photovoltaics. (Routledge, 2011).

Günes, S., Neugebauer, H. \& Sariciftci, N. S. Conjugated polymer-based organic solar cells. Chemical reviews 107, 1324-1338 (2007).

4 Xing, K. Z., Fahlman, M., Chen, X. W., Inganäs, O. \& Salaneck, W. R. The electronic structure of poly (3, 4-ethylene-dioxythiophene): studied by XPS and UPS. Synthetic Metals 89, 161-165 (1997).

Kittel, C. Introduction to solid state physics. ( John Wiley \& Sons, 1996).

Sze, S. M. Semiconductor devices: physics and technology. (John wiley \& sons, 2008). Kim, N. et al. in Conjugated Polymers: Properties, Processing, and Applications (CRC Press Taylor \& Francis Group, 2019).

8 Schubert, M. Infrared ellipsometry on semiconductor layer structures: phonons, plasmons, and polaritons. Vol. 209 (Springer Science \& Business Media, 2004). 
Gerhardt, R. Impedance and dielectric spectroscopy revisited: distinguishing localized relaxation from long-range conductivity. Journal of Physics and Chemistry of Solids $\mathbf{5 5}$, 1491-1506 (1994).

Dygas, J. R. Dielectric function of ionic conductors studied by impedance spectroscopy. Solid State lonics 176, 2065-2078 (2005).

21 Yang, X. et al. Permittivity of Undoped Silicon in the Millimeter Wave Range. Electronics 8, 886 (2019). Ahmad, Z. in Dielectric material (IntechOpen, 2012).

Lee, K., Heeger, A. J. \& Cao, Y. Reflectance of polyaniline protonated with camphor sulfonic acid: disordered metal on the metal-insulator boundary. Physical Review B 48, 14884 (1993).

Lee, K., Menon, R., Yoon, C. O. \& Heeger, A. J. Reflectance of conducting polypyrrole: Observation of the metal-insulator transition driven by disorder. Physical Review B $\mathbf{5 2}$, 4779 (1995).

Kim, N. et al. Role of interchain coupling in the metallic state of conducting polymers. Physical Review Letters 109, 106405 (2012). studies of the optical properties of doped poly (3, 4-ethylenedioxythiophene): an anisotropic metal. Thin Solid Films 313, 356-361 (1998).

Pettersson, L. A. A., Ghosh, S. \& Inganäs, O. Optical anisotropy in thin films of poly (3, 4-ethylenedioxythiophene)-poly (4-styrenesulfonate). Organic Electronics 3, 143-148 (2002).

Chen, S. et al. On the anomalous optical conductivity dispersion of electrically conducting polymers: ultra-wide spectral range ellipsometry combined with a Drude-Lorentz model. Journal of Materials Chemistry C 7, 4350-4362 (2019).

Aasmundtveit, K. E. et al. Structure of thin films of poly (3, 4-ethylenedioxythiophene). Synthetic Metals 101, 561-564 (1999).

molecules 33, 3120-3127 (2000).

31 Fujiwara, H. Spectroscopic ellipsometry: principles and applications. (John Wiley \& Sons, 2007).

32 Ashcroft, N. W., Mermin, N. D. \& Mermin, N. D. Solid State Physics. (Holt, Rinehart and Winston, 1976).

33 Datta, S. Electronic transport in mesoscopic systems. (Cambridge university press, 1997).

34 Fox, M. Optical properties of solids. (Oxford University Press, 2010).

35 Urbach, F. The long-wavelength edge of photographic sensitivity and of the electronic absorption of solids. Physical Review 92, 1324 (1953).

36 Pavunny, S. P., Thomas, R. \& Katiyar, R. S. Cauchy-urbach dielectric function modeling of amorphous high-k lagdo3 films. ECS Transactions 45, 219 (2012).

37 Chen, H. \& Shen, W. Z. Perspectives in the characteristics and applications of TaucLorentz dielectric function model. The European Physical Journal B-Condensed Matter and Complex Systems 43, 503-507 (2005). 


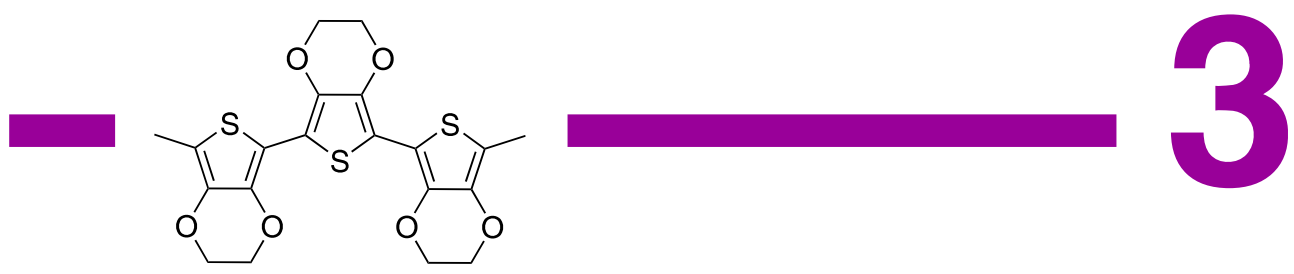

\section{Plasmonics and Structural Colours}

Plasmonics, associated with strong resonant oscillations of conducting-band electrons in metal nanostructures, provides a unique way to manipulate light-matter interactions at the nanoscale. In this chapter, we will briefly review two main types of plasmons as well as their basic properties. Potential candidates for plasmonic materials and their required properties are discussed. In the last section, we make a concise introduction of structural colours and different mechanisms or devices to create vivid structural colours.

\section{Contents}

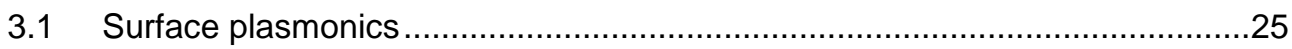

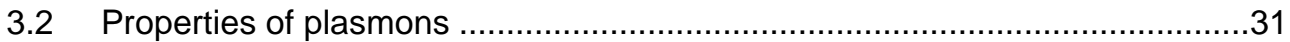

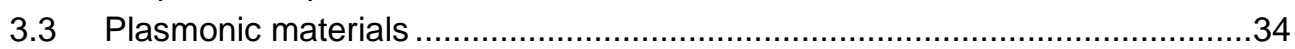

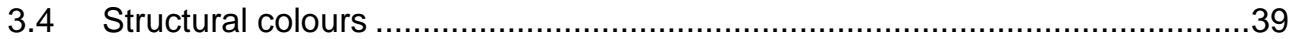

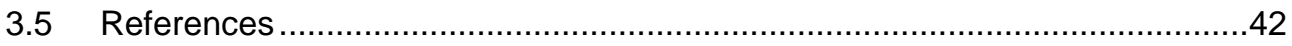

\subsection{Surface plasmonics}

We continue the discussion on the electromagnetic wave equation of Equation (2.8) in a material with permittivity of $\varepsilon$. The equation can be written in Fourier domain: ${ }^{1}$ 


$$
\mathbf{k}(\mathbf{k} \cdot \mathbf{E})-\mathbf{k}^{2} \mathbf{E}=-\varepsilon(\mathbf{k}, \omega) \frac{\omega^{2}}{c^{2}} \mathbf{E},
$$

where $\mathbf{k}$ is the wavevector (and its scalar quantity $k$ is the wavenumber). If the wave is a longitudinal wave (where $\mathbf{k}$ is parallel to the electric field $\mathbf{E}$ ), the equation becomes

$$
\varepsilon(\mathbf{k}, \omega)=0 .
$$

Solutions to the equation exist only when the material permittivity is zero. On the other hand, if the wave is a transverse wave, where $\mathbf{k}$ is perpendicular to $\mathbf{E}$, we have

$$
k^{2}=\varepsilon(\mathbf{k}, \omega) \frac{\omega^{2}}{c^{2}} .
$$

The permittivity of metals can be described by the simplified Drude model in Equation (2.52), and then the equation becomes

$$
\omega^{2}=c^{2} k^{2}+\omega_{p}^{2}
$$

The $k$ - $\omega$ dispersion revealed by Equation (3.4) is plotted in Figure 3.1. The equation only has solutions when the angular frequency is above the plasma frequency (orange curve), where the metal becomes transparent and the electromagnetic wave can travel through it. At the point of the plasma frequency, the permittivity is approximately zero, which also fits the case in Equation (3.2). In this special condition, the electric field in the metal is completely depolarized, with electric field of $-\mathbf{P} / \varepsilon_{0}$ according to Equations (2.6a) and (2.5a). This case can be described as a dipolar system where the electron cloud (represented by the shadow in the right part of Figure 3.1) is displacing away from the lattices of nucleus. The collective oscillation of electrons in this situation is longitudinal, and we define it as volume (or bulk) plasmon (turquoise curve in Figure 3.1 $)^{1,2}$.
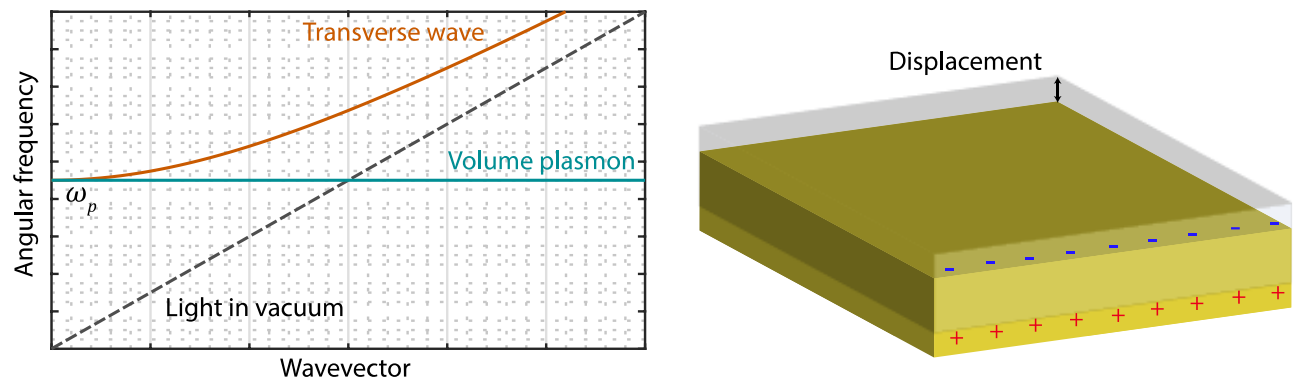

Figure 3.1 | Volume plasmon in metals. The left part presents the dispersion curves of electromagnetic waves propagating in metals, and volume plasmon is indicated by a turquoise line. Light in vacuum is presented by a grey dashed line. The right part schematically illustrates the dipole formation of volume plasmon where the electron cloud (grey shadow) is displacing away from nucleus (golden colour).

The word, plasmon, is used to describe the quasi-particle formed due to the collective oscillations of free electrons in the metal. Instead of bulk plasmons, most plasmonic systems are based on surface plasmons, which exhibit various attractive and unique properties. The two main types of surface plasmons will be introduced below. 


\subsubsection{Surface plasmon polaritons}

Surface plasmon polaritons can exist at the interface between a metal with permittivity $\varepsilon_{1}$ and a dielectric with permittivity $\varepsilon_{2}$ (see Figure 3.2). The electromagnetic wave at the interface is assumed to be propagating along the $x$-axis and with no field variation along the $y$-axis. The wave formula can be written as ${ }^{1}$

$$
\mathbf{E}(x, y, z)=\mathbf{E}(z) e^{i \beta x},
$$

where $\beta$ is the complex propagation constant for the wave (wavevector along $x$-axis). Using the Helmholtz equation (Equation (2.10)), we have ${ }^{1}$

$$
\frac{\partial^{2} \mathbf{E}(z)}{\partial z^{2}}+\left(k_{0}^{2} \varepsilon-\beta^{2}\right) \mathbf{E}(z) e^{i \beta x}=0 .
$$

According to Maxwell's Equations (2.4c) and (2.4d), two sets of solutions exist, either transverse magnetic (TM) mode or transverse electric (TE) mode. For TM mode: ${ }^{1}$

$$
\begin{gathered}
H_{x}=i \frac{1}{\omega \mu_{0}} \frac{\partial E_{y}}{\partial z}, \\
H_{z}=\frac{\beta}{\omega \mu_{0}} E_{y}, \\
\frac{\partial^{2} E_{y}}{\partial z^{2}}+\left(k_{0}^{2} \varepsilon-\beta^{2}\right) E_{y}=0 .
\end{gathered}
$$

Assuming that the electric field is ${ }^{1}$

$$
E_{y}(z)=A_{1} e^{i \beta x} e^{k_{1} z}
$$

in the metal (where $z<0$, see the right part of Figure 3.2), we have magnetic fields in the metal too: ${ }^{1}$

$$
\begin{gathered}
H_{x}(z)=i \frac{A_{1} k_{1}}{\omega \mu_{0}} e^{i \beta x} e^{k_{1} z}, \\
H_{z}(z)=\frac{A_{1} \beta}{\omega \mu_{0}} e^{i \beta x} e^{k_{1} z} .
\end{gathered}
$$

For the dielectric side of the interface $(z>0)$, we have similar expressions: ${ }^{1}$

$$
\begin{gathered}
E_{y}(z)=A_{2} e^{i \beta x} e^{-k_{2} z}, \\
H_{x}(z)=-i \frac{A_{2} k_{2}}{\omega \mu_{0}} e^{i \beta x} e^{-k_{2} z}, \\
H_{z}(z)=\frac{A_{2} \beta}{\omega \mu_{0}} e^{i \beta x} e^{-k_{2} z},
\end{gathered}
$$

where $A_{1}, A_{2}, k_{1}$, and $k_{2}$ are field amplitudes and wavevectors along the $z$-axis (indicating the decay of the field) for the metal (1) and the dielectric (2). At the interface $z=0$, the electric displacement $\mathbf{D}$ and the auxiliary magnetic field $\mathbf{H}$ should be identical and this gives ${ }^{1}$

$$
A_{2}\left(k_{1}+k_{2}\right)=A_{1}\left(k_{1}+k_{2}\right)=0 .
$$

The solution for Equation (3.9) only exists when the amplitudes for the electric fields at both sides are zero. Hence, TM modes are not supported at the interface. 
For TE mode, similar expressions as Equations (3.8) can be derived and utilizing the boundary conditions of identical electric displacement and $\mathbf{H}$-field at the interface, we can derive the relations: 1

$$
\begin{gathered}
A_{1}=A_{2}, \\
A_{1} k_{1}+A_{2} k_{2}=0 .
\end{gathered}
$$

Using the wave equation of TE mode ${ }^{1}$

$$
\frac{\partial^{2} H_{y}}{\partial z^{2}}+\left(k_{0}^{2} \varepsilon-\beta^{2}\right) H_{y}=0,
$$

and electric and magnetic field expressions, we obtain

$$
\begin{aligned}
& k_{1}^{2}=\beta^{2}-k_{0}^{2} \varepsilon_{1}, \\
& k_{2}^{2}=\beta^{2}-k_{0}^{2} \varepsilon_{2} .
\end{aligned}
$$

Combining the relations revealed in Equation (3.10), we obtain the expression for $\beta$ :

$$
\beta=k_{0} \sqrt{\frac{\varepsilon_{1} \varepsilon_{2}}{\varepsilon_{1}+\varepsilon_{2}}} .
$$

Using the simplified Drude model (Equation (2.52)) for the metal and $\varepsilon_{2}=1$ for the dielectric (assuming air), we obtain the dispersion curve presented in Figure 3.2.
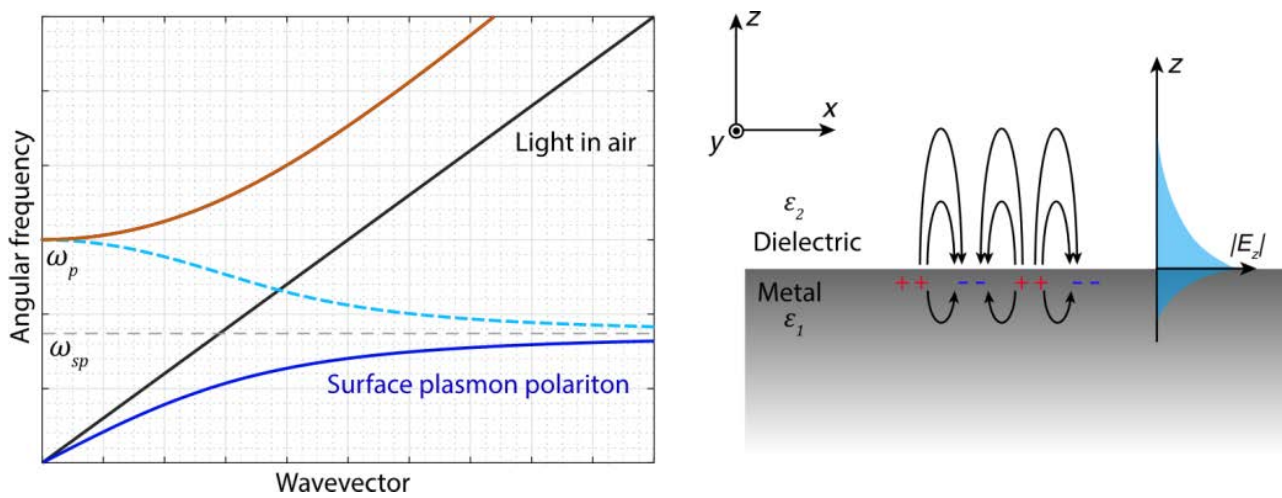

Figure 3.2 | Surface plasmon polaritons at metal/dielectric interfaces. The left part is the dispersion curve for surface plasmon polaritons (blue solid curve) below the surface plasmon frequency. The blue dashed curve between the plasma and surface plasmon frequencies indicates the range where the propagation constant is an imaginary number. The right part schematically presents the nature of surface plasmon polaritons: coupling of electrostatic charges and electro-magnetic wave travelling along the interface. The amplitude of the electric field $\left|E_{z}\right|$ decays rapidly away from the interface. The figure is modified based on literature ${ }^{3}$.

At a certain frequency where the permittivity sum of the metal $\left(\varepsilon_{1}=-1\right)$ and the dielectric $\left(\varepsilon_{2}=1\right)$ is zero, the propagation constant $\beta$ approaches infinity. This frequency is the surface plasmon frequency $\omega_{s p}$, and can be calculated by ${ }^{1}$

$$
\omega_{s p}=\frac{\omega_{p}}{\sqrt{1+\varepsilon_{2}}},
$$


where for air as the dielectric, the frequency is about $0.71 \omega_{p}$. The group velocity of the wave can be calculated by the slope of the dispersion curve at a certain frequency. At the surface plasmon frequency, the group velocity is zero, indicating the electrostatic character of the wave. At ultralow frequencies, the group velocity of the wave at the interface is similar to that of light in air. At high frequencies approaching $\omega_{s p}$, the bent shape of the dispersion curve indicates a slower group velocity and a bounded electric field at the interface (larger wavevectors and smaller wavelengths compared to those in air). Therefore, the surface plasmon could squeeze or confine the electromagnetic wave (or light) into a far smaller length scale.

At a given frequency, the momentum of the surface plasmon polariton is higher than that of light in the air and it is therefore difficult for the light beam to directly excite the plasmons. Surface plasmon polaritons can instead be excited via beams of charged particles or special coupling techniques utilizing prisms or gratings ${ }^{1}$.

The above discussion used the simplified Drude model and did not consider damping caused by collisions ( $i \omega \gamma$ ) or interband transitions. In the real case, there is a maximum value instead of infinity for the wavevector at the surface plasmon frequency and the imaginary wavevector (dashed curves in Figure 3.2) which was forbidden in previous case is allowed $(|\boldsymbol{\beta}| \neq 0)$.

\subsubsection{Localized surface plasmons}

The surface plasmon polariton modes become quantized when the scale of the thin film is further shrunk into three-dimensional nanostructures, such as nanoparticles and nanohole arrays. We start the discussion from a single metal nanosphere with radius of $a$ (Figure 3.3). For simplicity, we use the quasi-static approximation here that the size of the particle is much smaller than the wavelength of the light used ${ }^{1}$. Therefore, we can calculate the electric potential distribution $\psi$ inside and outside the particle under an electric field $E_{0}$ through the Laplace equation ${ }^{1}$

$$
\nabla^{2} \psi=0,
$$

since there is no net charge in the system. We set the center of the nanosphere as the origin, and introduce a polar coordinate system with $r$ as the radial coordinate and $\theta$ as the angular coordinate (see Figure 3.3). The solutions for Equation (3.15) are ${ }^{1}$

$$
\begin{gathered}
\psi_{\text {inside }}(r, \theta)=\sum_{l=0}^{\infty} A_{l} r^{l} P_{l}(\cos \theta), \\
\psi_{\text {outside }}(r, \theta)=\sum_{l=0}^{\infty}\left[B_{l} r^{l}+C_{l} r^{l-1}\right] P_{l}(\cos \theta),
\end{gathered}
$$

where $A_{l}, B_{l}, C_{l}$ are parameters and $P_{l}(\cos \theta)$ are the Legendre Polynomials of order $l^{1}{ }^{1}$ Using the boundary conditions for positions at infinity $(r=\infty)$ and at the surface of the particle $(r=a)$, we can obtain the solution ${ }^{1}$

$$
\psi_{\text {inside }}(r, \theta)=-\frac{3 \varepsilon_{2}}{\varepsilon(\omega)+2 \varepsilon_{2}} E_{0} r \cos \theta,
$$




$$
\psi_{\text {outside }}(r, \theta)=-E_{0} r \cos \theta+\frac{\varepsilon(\omega)-\varepsilon_{2}}{\varepsilon(\omega)+2 \varepsilon_{2}} E_{0} a^{3} \frac{\cos \theta}{r^{2}} .
$$

For Equation (3.17b), the second part on the right side can be regarded as the effect exerted by a dipole formed in the nanoparticle (electron cloud is displacing away from the positive nucleus lattices, see the right part of Figure 3.3). It can be written as ${ }^{1}$

$$
\frac{\varepsilon(\omega)-\varepsilon_{2}}{\varepsilon(\omega)+2 \varepsilon_{2}} E_{0} a^{3} \frac{\cos \theta}{r^{2}}=\frac{\mathbf{P} \cdot \mathbf{r} \cos \theta}{4 \pi \varepsilon_{0} \varepsilon_{2} r^{3}} .
$$

Therefore, we have the expression for the polarization $\mathbf{P}$

$$
\mathbf{P}=4 \pi \varepsilon_{0} \varepsilon_{2} a^{3} \frac{\varepsilon(\omega)-\varepsilon_{2}}{\varepsilon(\omega)+2 \varepsilon_{2}} \mathbf{E}_{0} .
$$

We define the polarizability $\alpha$ of the nanoparticle as ${ }^{1}$

$$
\alpha=\frac{\mathbf{P}}{\varepsilon_{0} \varepsilon_{2} \mathbf{E}_{0}}=4 \pi a^{3} \frac{\varepsilon(\omega)-\varepsilon_{2}}{\varepsilon(\omega)+2 \varepsilon_{2}} .
$$
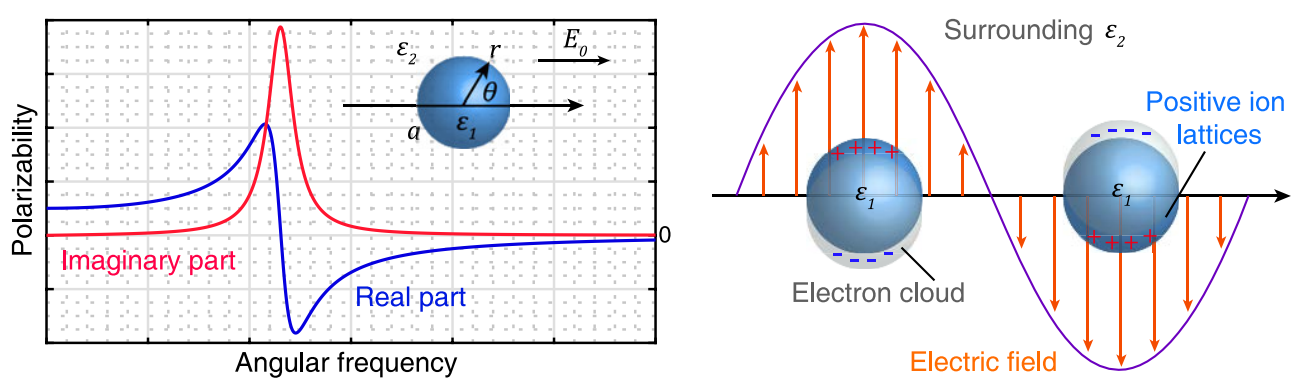

Figure 3.3 | Localized surface plasmons. The left part indicates the polarizability-frequency relation for a nanosphere (permittivity: $\varepsilon_{1}$ ) with diameter of $a$ in a medium with permittivity of $\varepsilon_{2}$. The real (blue) and imaginary (red) parts are indicated in the figure. The right part shows a schematic of localized surface plasmons, where dipolar resonance is induced by a time-varying electric field.

Using the Drude model (Equation (2.43)) for the metal permittivity, we can derive the polarizability of the nanosphere (Figure 3.3). A maximum value of the polarizability exists at the frequency where the denominator $\left(\varepsilon(\omega)+2 \varepsilon_{2}\right)$ in Equation (3.20) is minimized. For air as the dielectric, the angular frequency for the resonance is

$$
\omega_{s p}=\frac{\omega_{p}}{\sqrt{1+2 \varepsilon_{2}}}=\frac{\omega_{p}}{\sqrt{3}} .
$$

This condition leads to the formation of localized (dipolar) surface plasmons, where the negatively charged electron cloud is displacing from the positively charged lattices of ions, and oscillating collectively in response to the external electric field of light.

From the polarizability, we can obtain the optical scattering and extinction crosssections: ${ }^{1}$

$$
C_{s c a}=\frac{k^{4}}{6 \pi}|\alpha|^{2}=\frac{8 \pi}{3} k^{4} a^{6}\left|\frac{\varepsilon(\omega)-\varepsilon_{2}}{\varepsilon(\omega)+2 \varepsilon_{2}}\right|^{2},
$$




$$
C_{\text {ext }}=k \operatorname{Imag}(\alpha)=4 \pi k a^{3} \operatorname{Imag}\left(\frac{\varepsilon(\omega)-\varepsilon_{2}}{\varepsilon(\omega)+2 \varepsilon_{2}}\right) .
$$

The expression for polarizability can be further generalized for an oblate spheroid:1,4

$$
\alpha_{L_{i}}=V \frac{\varepsilon(\omega)-\varepsilon_{2}}{\varepsilon_{2}+L_{i}\left(\varepsilon(\omega)-\varepsilon_{2}\right)},
$$

where $\mathrm{V}$ is the volume of the spheroid and the $L_{i}$ is a geometrical factor: ${ }^{1}$

$$
L_{i}=\frac{a_{1} a_{2} a_{3}}{16} \int_{0}^{\infty} \frac{d q}{\left(a_{i}^{2} / 4+q\right) f(q)},
$$

where $i$ corresponds to the selected direction along the spheroids three axes, $a_{1}, a_{2}$, and $a_{3}$ are the lengths of the spheroid's three axes and $f(q)$ is a function expressed as $^{1}$

$$
f(q)=\sqrt{\left(q+a_{1}^{2} / 4\right)\left(q+a_{2}^{2} / 4\right)\left(q+a_{3}^{2} / 4\right)} .
$$

In this thesis, we used the above expressions for oblate spheroids to approximate the optical response of nanodisks. By changing the aspect ratio of the disks (from rod to disk), the geometric factor can vary from 0.5 to $0 .{ }^{4}$ Disks with large diameters requires corrections from the quasi-static approximation, which can be addressed using a modified polarizability $\alpha^{\prime}$ described as ${ }^{5,6}$

$$
\alpha^{\prime}=\alpha\left[1-\frac{k^{2}}{2 \pi D} \alpha-i \frac{k^{3}}{6 \pi} \alpha\right]
$$

where $D$ is the diameter of the nanodisks. Combining Equation (3.26) and (3.22b), we can theoretically calculate the extinction cross-section of the nanodisks.

Localized surface plasmons can be directly excited via free space light. The properties of localized surface plasmons are affected by various factors including the geometry of the nanoparticles and their surrounding medium, which we will illustrate below.

\subsection{Properties of plasmons}

Although the detailed theory of plasmonics was established within the last 50 years, the application of plasmonics started more than 2000 years ago, such as the Lycurgus Cup used by ancient Roman7. In this section, we will focus on the properties and potential (or matured) applications of surface plasmons.

\subsubsection{Confinement and enhancement of the electric field}

One of the attractive properties of surface plasmons is that it can effectively confine the optical field in an ultrasmall space (at the surface or around a nanoparticle) leading to an enhanced field intensity.

For surface plasmon polaritons, the propagation length can be calculated by ${ }^{1}$

$$
L=1 /(2 \operatorname{Imag}(\beta)),
$$


and the penetration depth can be calculated by ${ }^{1}$

$$
z=1 /\left|\operatorname{Imag}\left(k_{1}\right)\right| \text {. }
$$

For a silver/air interface, a wavelength of $450 \mathrm{~nm}$ can yield $L$ of $16 \mu \mathrm{m}$ and $z$ of 180 $n \mathrm{~m}$, while a wavelength of $1.5 \mu \mathrm{m}$ gives $L$ of $1080 \mu \mathrm{m}$ and $z$ of $2.6 \mu \mathrm{m} .{ }^{1}$ Although there is a trade-off between the propagation and confinement (penetration depth), this property makes surface plasmon polaritons have the potential for waveguide applications $^{8}$ and ultracompact lasers.

Excitation of localized plasmons also lead to strong field enhancements, which depend on the geometry of the nanostructure and the exact position. This enhancement effect is crucial for applications like surface-enhanced Raman scattering ${ }^{9,10}$ and plasmonenhanced fluorescence spectroscopies ${ }^{11}$. In addition, the field enhancement can be boosted by coupled nanostructures (e.g., dimers) and hot spots that are formed in the area between them ${ }^{12}$. This enhancement ${ }^{13}$ also results in promising applications like photo-catalysis, superlensing, near-field nano-imaging, and non-linear optics ${ }^{14,15}$.

\subsubsection{Precise control of scattering and absorption}

The optical properties of metal nanoparticles can be precisely tailored by its geometric parameters, including size and shape. For example, increasing the aspect ratio of a nanorod (nano-rice or nano-cuboid), leads to red-shift of the scattering peak (Figure 3.4) ${ }^{17}$. With the advancement of nano-chemistry, researchers are able to synthesize particles with desired shapes, included but not limited to spheres, ellipsoids, prisms, and stars ${ }^{16}$. The tuning of these parameters give rise to additional degrees of freedom in the control of plasmonic properties for various applications.
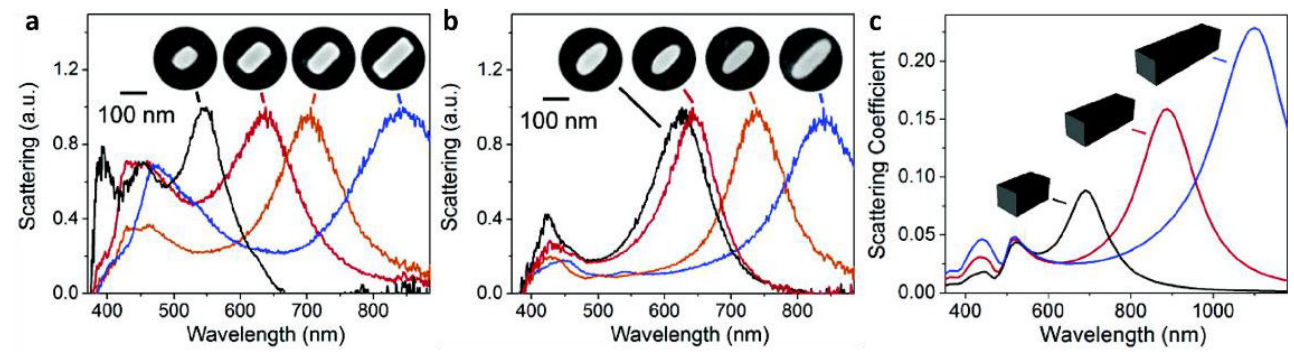

Figure 3.4 | Examples of red-shifted scattering peak for metal nanostructures with increasing aspect ratio. a, Nanorods, b, nano-rice, c, nano-cuboids with different aspect ratios. The figure is reproduced from Reference 17 with permission from American Chemical Society.

The precise control of light scattering is beneficial for applications like nanoantennas. A nanoantenna is a device that efficiently makes conversions between optical signals and confined electrical signals ${ }^{18}$. Nanoantennas based on plasmonics show promising possibilities, including unidirectional emission control of nano-emitters ${ }^{19}$, broadband light bending ${ }^{20}$, and tuning the frequency response of scattering ${ }^{21}$. 
Design concepts incorporating plasmonics have been used in both photovoltaics and light-emitting diodes. Plasmonic nanoparticles could trap or guide light by scattering at the interface layers in these optoelectronic devices ${ }^{22}$. Recently, it was demonstrated that randomly arranged plasmonic structures improved the device stability of organic light emitting diodes by decay rate enhancement ${ }^{23}$. Thus, plasmonics hold possibilities to boost both efficiency and stability for high-performance optoelectronic devices.

The absorption and scattering of localized plasmons are also affected by the medium or materials that surrounds the metal nanostructure, as indicated by $\varepsilon_{2}$ in Equation (3.22). This enables their use in (bio-)molecule sensing, $\mathrm{pH}$ sensing, refractometric sensing, and possibility of being integrated into microfluidic devices ${ }^{24}$. In spite of several remaining issues ${ }^{25}$, some applications have already been commercialized as a mature product ${ }^{26}$.

Tuning of plasmonic resonances via geometry of metal nanostructures can also be utilized for colour generation, which will be discussed in Section 3.4.

\subsubsection{Losses and local heating effects}

Interband and intraband transitions (described by the imaginary part of the permittivity) constitute two main loss mechanisms for plasmonics based on metals and other materials ${ }^{27}$. Non-radiative decay of plasmons by these losses results in local heat released by plasmonic nanostructures, with temperature distribution depending on the size and geometry of the nanostructure and the thermal conductivity of the surroundding medium.

Local plasmonic heating has been explored for various applications. It has been proposed for medication, where via the heating effect initiated by light (photothermal effect), plasmonic nanoparticles could kill cancerous or tumor cells ${ }^{28}$. Modifying chemical reactions is another possible application, where the elevated temperature at specific sites can facilitate and enhance reaction kinetics towards desired products ${ }^{29}$. Plasmonic nanoparticles have also been reported for solar steam generation, where the local heating by nanoparticles facilitate water evaporation converting a polluted solution into pure distilled water ${ }^{30}$. Plasmonic local heating has also been utilized for nanowire junction welding for semiconductor devices ${ }^{31}$.

Another possibility is to integrate local plasmonic heating into devices based on other effects like thermoelectric and pyroelectric effects (Figure 3.5). For example, heat generated by plasmons was used to create a temperature gradient in aligned nanowires made of $\mathrm{Sb}_{2} \mathrm{Te}_{3}$, a high-efficiency thermoelectric material ${ }^{32}$. By tuning the geometric factors of the nanowires (e.g., width, pitch, or height), the devices showed high sensitivity to light of different wavelengths and generated photo-thermal voltages. Such systems could be used for optical detection. Similar applications have been explored using hybrid devices combining plasmonic nanostructures with ionic thermoelectric capacitors and pyro-electric generators (Figure 3.5) ${ }^{33-36}$. 
a

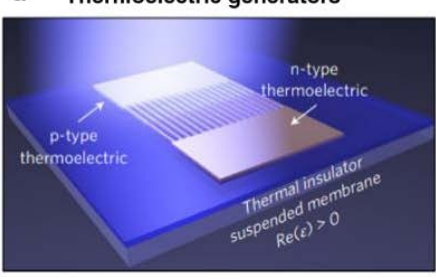

b

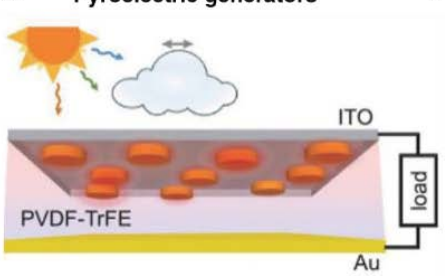

C Ionic thermoelectric capacitors

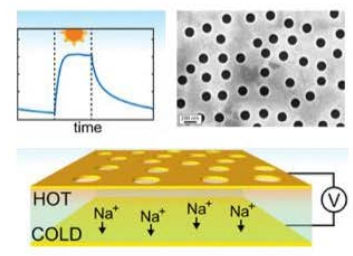

Figure 3.5 | Hybrid devices based on local heating of localized plasmons. a, Thermoelectric generators, b, pyroelectric generators, c, ionic thermoelectric capacitors. The figures are reproduced from Reference 32, 33, and 34 with permission from Springer Nature, American Chemical Society, and John Wiley and Sons.

\subsubsection{Hot carriers}

Non-radiative decay of surface plasmons creates hot carriers in the materia| ${ }^{37}$. Hot carriers are defined as the carriers (electrons or holes) with energies larger than those of thermal excitations at ambient temperatures ${ }^{37}$. The hot carrier concept can be employed in new types of functional devices. For example, the cooling process of hot carriers enables plasmonic solar cells and photodetectors based on plasmon-enhanced field-effect transistors ${ }^{38,39}$. Hot carriers can also be used for catalysis and sensing applications ${ }^{40,41}$.

\subsection{Plasmonic materials}

Currently, the dominant materials for plasmonics are traditional metals, especially noble metals, like gold and silver. The framework of metallic plasmonics have been well established and there have been numerous studies varying from fundamental optical properties to potential applications ${ }^{1,42,43}$. Other metals like aluminum, copper, platinum, magnesium, and palladium are also explored for their unique applications for UV detection, photo-catalysis, hydrogen sensing, and dynamic colour generation ${ }^{6,44-48}$. However, it should be noted that metals have some challenges limiting their further applications. Typically, different metals have their own issues, such as losses by interband transitions for gold, and chemical instability for aluminum and silver ${ }^{27}$. The compatibility with current silicon technology and fixed optical parameters are common challenges for metals ${ }^{27}$. Therefore, finding alternatives to metals for specific plasmonic applications is highly demanded and has become one of the main research directions in nano-optics ${ }^{27,49-51}$.

Based on the definitions of surface plasmons by Equation (3.13) and (3.20), the prerequisites to generate plasmonic resonances in a material is

$$
\text { Real }(\varepsilon(\omega))<-1 .
$$

In addition, the imaginary part of permittivity should be small compared to its real counterpart to avoid large dissipation of the resonance: 


$$
\left|\frac{\operatorname{Imag}(\varepsilon(\omega))}{\operatorname{Real}(\varepsilon(\omega))}\right|<1 .
$$

Therefore, we defined a plasmonic regime as a basic criterion for plasmonic materials via Equations (3.29) and (3.30). According to optical models introduced in Section 2.3, the Drude model for conductors produces a broad range of negatively valued real permittivity. The first category of potential plasmonic materials would be conductors. In addition, the Lorentz oscillator shows a relatively narrow range with negative real permittivity in its high energy side of the resonance energy peak position (Figure 2.10). This means that materials with certain physical transitions, such as exciton creation or molecular (phonon) vibrations, may also be compatible with plasmon-like resonances. In this section, we pay attention to conduc-tors and potential organic materials for plasmonics and plasmon-like systems.

\subsubsection{Inorganic materials and two-dimensional materials}

Inorganic conductors can possess suitable permittivity to act as plasmonic materials in certain spectral ranges, depending on the requirements of the specific application. For exam-ple, conductive oxides, such as tin-doped indium oxide (ITO) and aluminumdoped zinc oxide (AZO), are the mostly used inorganic conductors for optoelectronic devices in visible and IR ranges. Compared to noble metals, the advantage of these oxides is that their optical or electric properties (charge density) could be tailored by deposition parameters ${ }^{52,53}$. Altering the deposition temperature (Figure 3.6a) or the spacing between vertically aligned nanorods (similar as metallic nanostructures, see Figure 3.6b), could precisely shift the plasmonic resonances ${ }^{52,53}$.

Another class of materials that can have plasmonic properties is degenerate semiconductors, such as heavily doped silicon and germanium. Due to the limitation of doping density, the resonance range remains in the MIR range and pushing it into NIR or higher frequency range is a challenge ${ }^{27}$. Using semiconducting quantum dots like $\mathrm{Cu}_{2-\mathrm{x}} \mathrm{S}$, the resonances can be shifted into the NIR range by increasing the amount of copper vacacncies ${ }^{54}$. Electrochemical redox tunability was recently demonstrated in these plasmonic quantum dots ${ }^{55}$.

Transition metal nitrides, such as zirconium nitride and titanium nitride, form a class of refractory plasmonic materials that show high thermal stability compared to metals ${ }^{27}$. Nanostructures of these materials can exhibit plasmonic resonance in the visible and NIR ranges. Broadband absorbers were realized by a periodic lattice of square, ringlike unit cells of titanium nitrides (see Figure 3.6c) ${ }^{56}$. These materials are also named plasmonic ceramics, together with rare-earth perovskite conductors like strontium molybdate and strontium niobate ${ }^{58}$.

Graphene has emerged as a promising plasmonic material since its first discovery in $2004^{59}$. The unique semi-metallic behaviour and band structure in graphene lead to opportunities for electro-optical modulation, optical sensing, quantum plasmonics, and light harvesting ${ }^{60}$. Using near-field scanning optical microscopy, the propagation of surface plasmon polaritons was observed in graphene ${ }^{61,62}$. Making graphene into a 
nano-ribbon array can tune its localized plasmonic resonance in the MIR range, which is suitable for biosensing (Figure $\mathbf{3 . 6 \mathbf { d }})^{57}$. The localized plasmon resonance can also be tuned by electrolyte gating, making graphene a novel platform for light modulation and switching applications ${ }^{63-65}$.
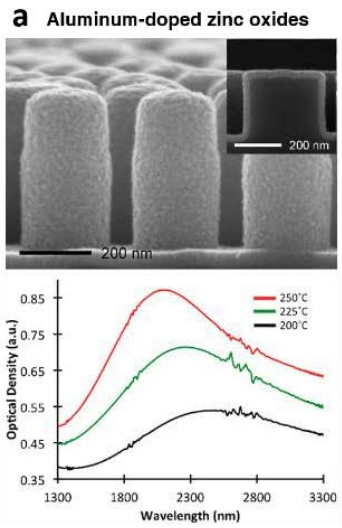
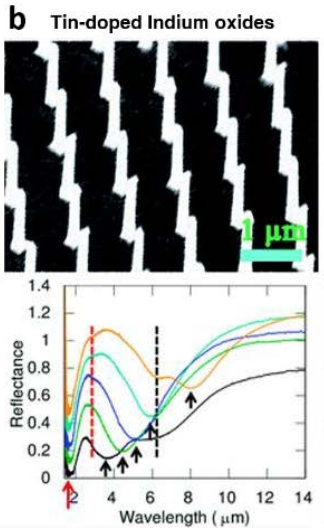
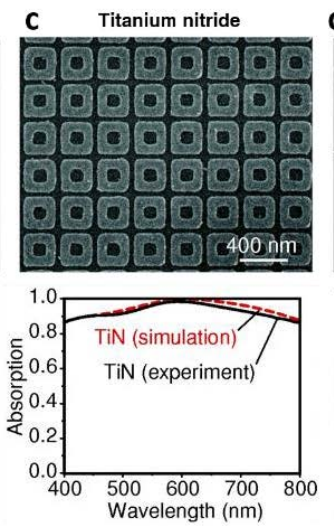
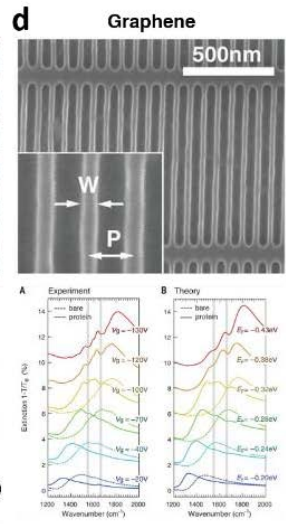

Figure 3.6 | Inorganic and carbon-based plasmonic materials. The figures are reproduced from Reference 52, 53, 56, and 57 with permission from John Wiley and Sons, American Chemical Society, and the American Association for the Advancement of Science.

\subsubsection{Potential of organic materials for plasmonics}

Organic materials are abundant, low-cost, flexible, solution-processable, and available for large scale fabrication. With the success of measuring optical parameters of heavily doped polypyrrole in the $\mathrm{THz}$ range, negative-valued real permittivity of polypyrrole was regarded as evidence of metallic behaviour in this range ${ }^{66}$. Two-dimensional aperture arrays of polypyrrole were fabricated and optical measurements presented clear "anomalous transmission" peaks (Figure 3.7a). These peaks were believed to be induced by surface plasmon polaritons in this perforated polymer film, agreeing with their theoretical calculation (Figure 3.7a). The permittivity in the study was extracted from $\mathrm{THz}$ time-domain spectroscopy for a very narrow range (0.1-0.6 THz) using the Drude model. However, our study indicated that measurement in ultranarrow range might not be reliable (see Paper1) and the permittivity derivation could be errorneous ${ }^{67}$. Conducting polymer, such as PEDOT used in our study (Paper 1 and 3), normally possesses ultra-large positive permittivity in the $\mathrm{THz}$ range ${ }^{4,67,68}$. Thus, it is difficult to identify the origin of these "anomalous transmission" peaks in the study.

Theoretical calculations indicated that polycyclic aromatic hydrocarbons could have tunable plasmonic resonances ${ }^{69}$. These organic molecules can be directly obtained as nanostructures from synthesis without post-fabrication and expected to be free of nanostructural imperfections (Figure 3.7b). Following studies confirmed the existence of molecular plasmons and utilized this for electrochromic devices ${ }^{70,71}$. 
Organic small molecules and dye molecules were introduced in 2013 as possible materials for plasmonics ${ }^{72}$. It was found that the permittivity dispersion of ZnTPP, a dye, is in the shape of Lorentz oscillator providing a plasmonic region in the visible. By prism coupling, a surface plasmonic polariton signal was believed to be observed ${ }^{72}$. However, the nature of the signal was argued to be surface exciton polaritons instead of plasmonic polaritons ${ }^{73}$. The lack of free charge carriers in these insulating materials indicated that only excitons instead of mobile electrons or holes were coupling with light (Figure 3.7c $)^{73,74}$.
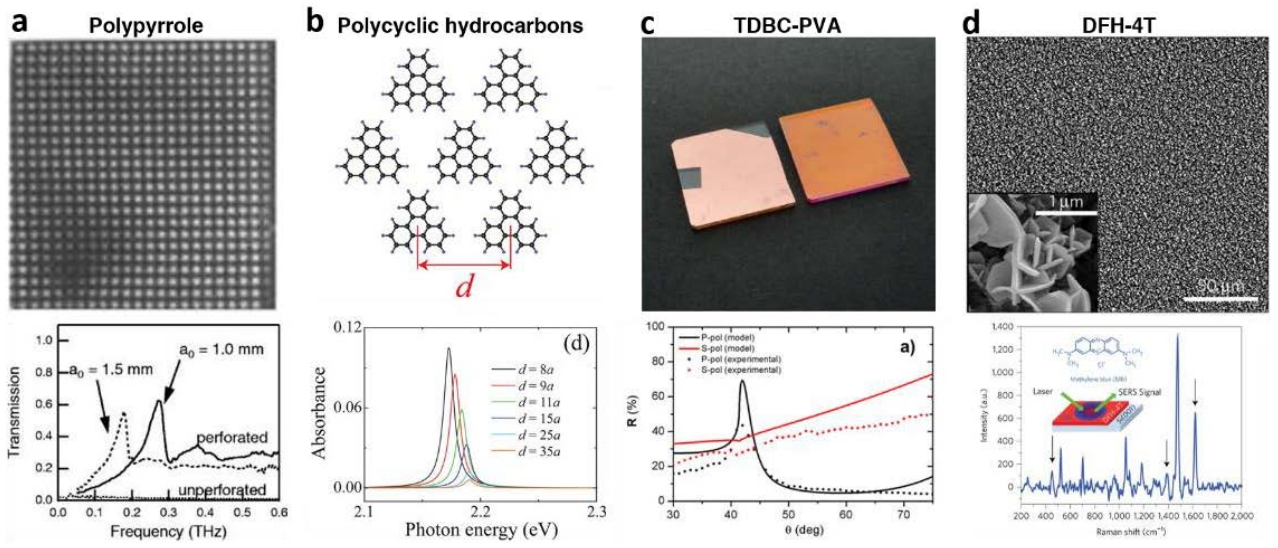

Figure 3.7 | Potential organic materials for plasmonics. The figures are reproduced from Reference 66, 69, 73, and 75 with permission from AIP Publishing, American Chemical Society, and Springer Nature.

Recently, it was demonstrated that the $\pi$-conjugated organic semiconductor, DFH-4T, can be used as a platform for surface-enhanced Raman spectroscopy, without need of noble metal nanostructures (Figure 3.7d) (5) $^{7}$ The enhanced performance with these nano-structured organic semiconductors was similar to plasmonic effects, but was finally proved to be the result of a charge-transfer transition between molecules and organic semicondcutors ${ }^{75}$.

Despite these key studies in exploring organic materials for plasmonic and plasmonlike behavior, further research is needed to fully understand and utilize advantages of organic materials including conducting polymers for different applications. It should be noted that organic materials typically possess larger losses, and thus will likely not be used to replace metals or inorganics in traditional applications. Finding niche applications that make full use of some of these materials' unique redox state tunability should be the future focus of organic plasmonics research, showing promise for spatiotemporally tunable nano-optical systems.

\subsubsection{Spatiotemporal tunability of plasmonic systems}


Achieving spatiotemporal tunability in an optical system enables dynamical control of light-matter interactions, and thus is regarded as a critical feature for next-generation plasmonic materials ${ }^{76}$. As we demonstrated in previous sections, currently dominant plasmonics based on metals is lacking tunability since after fabrication the material properties are fixed and cannot be adjusted. Therefore, an additional requirement for potential plasmonic materials would be the tunability of material optical properties. There are already many excellent studies on possible tuning mechanisms (Figure 3.8) and we will introduce some of these pioneering works below.

Introducing flexible and stretchable substrates enables mechanical tuning of positiondependent phase discontinuities ${ }^{77}$ or spacing between nano-antennas ${ }^{78}$ in plasmonic systems (Figure 3.8a). In addition, refractive index changes of the surroundings form another approach to tune plasmonic scattering signals and to produce different colours (Figure 3.8c) ${ }^{79}$. However, these two approaches are passive tuning methods, where the plasmonic materials themselves do not change their states.

Possible active plasmonic materials include magnesium, where plasmonic systems based on magnesium nanostructures showed reversibility between its oxides and hydrides (Figure $\mathbf{3 . 8 b})^{47,48}$. In spite of a relatively long response time, multi-colour reflective display with magnesium nanoparticles presented dynamic colour erasing and restoring behavior ${ }^{48}$. In addition, graphene plasmonic structures showed tunability by electrolyte gating and the resonance could be tuned in the whole MIR range, although the extinction was not high ${ }^{64}$.
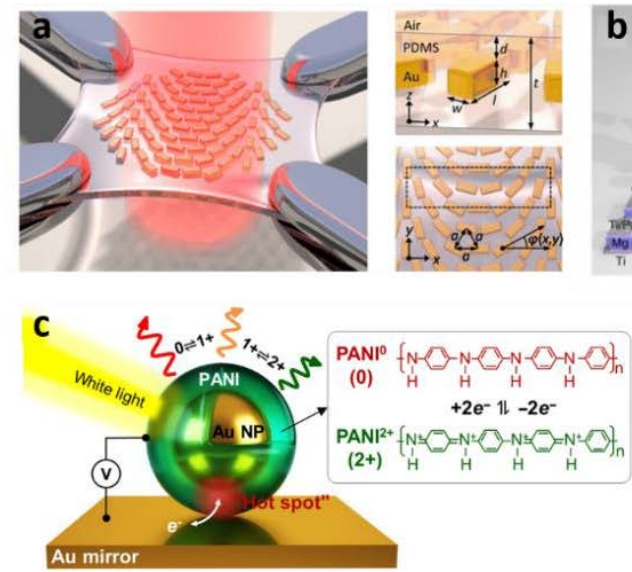
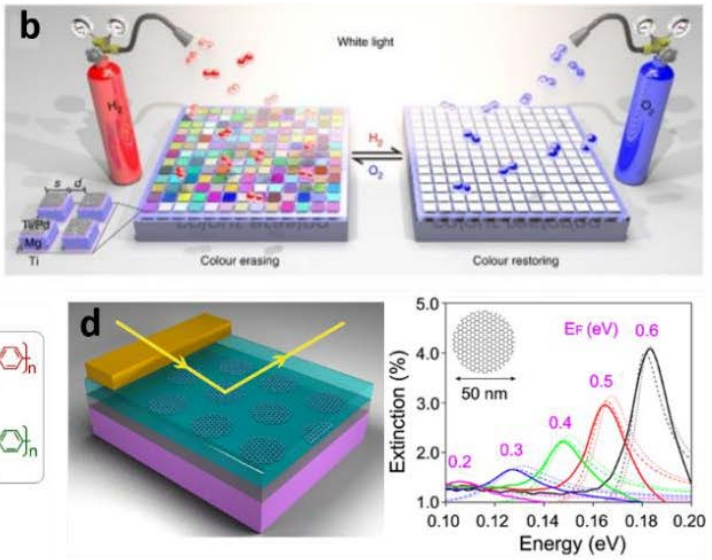

Figure 3.8 | Spatiotemporal tuning mechanisms for plasmonic systems. The figures are reproduced from Reference 48, 64, 77, and 79 with permission from American Chemical Society, Springer Nature, and the American Association for the Advancement of Science.

In this thesis, we attempted to exploit the redox-tunability of conducing polymers for dynamic plasmonics (more details in Section 6.3). 


\subsection{Structural Colours}

The generation of colour is a typical example of light-matter interactions, where light of certain wavelengths is absorbed, reflected, or transmitted, and captured by human eyes. Instead of colours produced by emissive devices, we here discuss reflective or transmissive colours where the optical material itself is not a light source. In this section, we focus on colours that are generated via material microstructures, instead of pigments that absorb light due to their chemical properties. Bird feathers and butterfly wings are typical examples of such structural colours in nature.

\subsubsection{Colour and its parameters}

The visualization of colours by human eyes is associated with the stimulation of photoreceptor cells (cone cells) that are sensitive to light at different wavelengths, i.e., blue, green, and red ${ }^{80}$. Thus, peak or dip wavelengths in reflection or transmission spectra becomes a potential parameter to describe colour. However, for some materials, multiple peaks co-exist in the spectra and thus, a more analytical approach to describe colour is demanded. In 1931, the International Commission on Illumination (Commission Internationale de l'eclairage) designed and published the first colour coordinate system (space) to describe the light of the whole visible range ${ }^{81}$.

In this system, a colour matching function (or tristimulus value curve) is created, where the spectral sensitivities equivalent to three primary colours are represented by $S_{X}, S_{Y}$, and $S_{Z}$ (Figure 3.9). For reflected light, the $X, Y$, and $Z$ coordinates is calculated by ${ }^{80}$

$$
\begin{aligned}
& X=\frac{1}{K} \int_{\lambda_{\min }}^{\lambda_{\max }} R(\lambda) I(\lambda) S_{X}(\lambda) d \lambda, \\
& Y=\frac{1}{K} \int_{\lambda_{\min }}^{\lambda_{\max }} R(\lambda) I(\lambda) S_{Y}(\lambda) d \lambda, \\
& Z=\frac{1}{K} \int_{\lambda_{\min }}^{\lambda_{\max }} R(\lambda) I(\lambda) S_{Z}(\lambda) d \lambda,
\end{aligned}
$$

where $\lambda_{\min }$ and $\lambda_{\max }$ are the minimal $(380 \mathrm{~nm})$ and maximal wavelengths $(750 \mathrm{~nm})$ of visible light, $R(\lambda)$ is the reflectance spectrum, and $I(\lambda)$ is the light source spectrum. $K$ is defined as ${ }^{80}$

$$
K=\int_{\lambda_{\min }}^{\lambda_{\max }} I(\lambda) S_{Y}(\lambda) d \lambda .
$$

The chromaticity of the material in CIE 1931 colour space is defined by two derived parameters, $x$ and $y$ (Figure 3.9):81,82

$$
\begin{aligned}
& x=\frac{X}{X+Y+Z}, \\
& y=\frac{Y}{X+Y+Z} .
\end{aligned}
$$

The overall shape of visible colours distributed in CIE 1931 colour space (Figure 3.9) is like a locus and each colour can find their specific position in it. CIE 1931 is the most 
commonly used way to determine colour. There are also other colour systems like CIE 1964 colour space, CIE RGB colour space, and CIE LAB colour space, which were not adopted in this thesis.
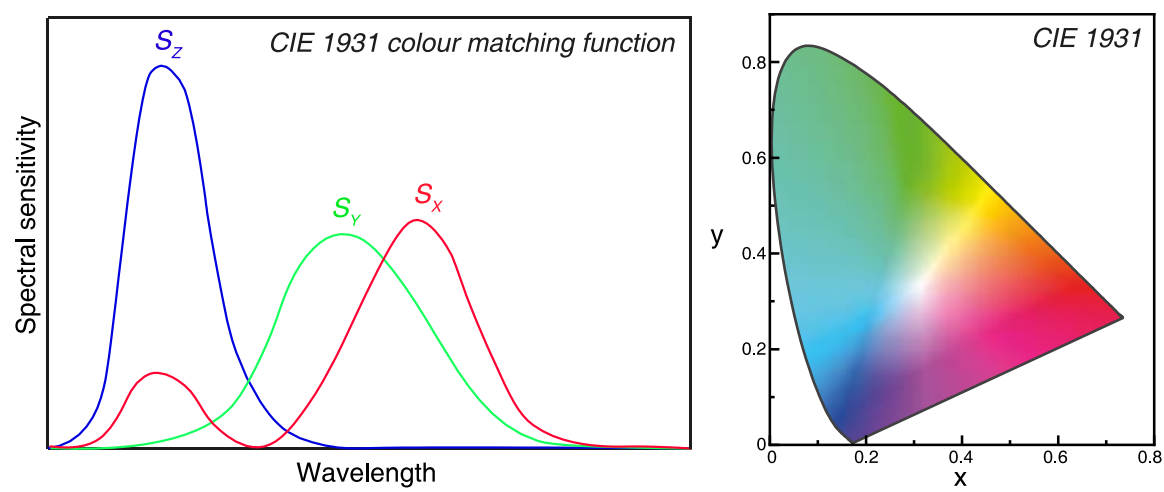

Figure 3.9 | CIE response functions and CIE 1931 xy colour coordinate system. The left panel is the tristimulus value curves for $S_{x}, S_{y}$, and $S_{z}$ in the CIE 1931 colour system. The right panel is the CIE 1931 xy colour space. Every colour can find its corresponding coordinate in the diagram. The figure is modified from literature ${ }^{83}$.

\subsubsection{Principles of structural colour generation}

There are various ways to create structural colours using different nano- to microscale structures to tailor the absorption and reflection of incident light. Some of these mechanisms are shown in Figure 3.10.
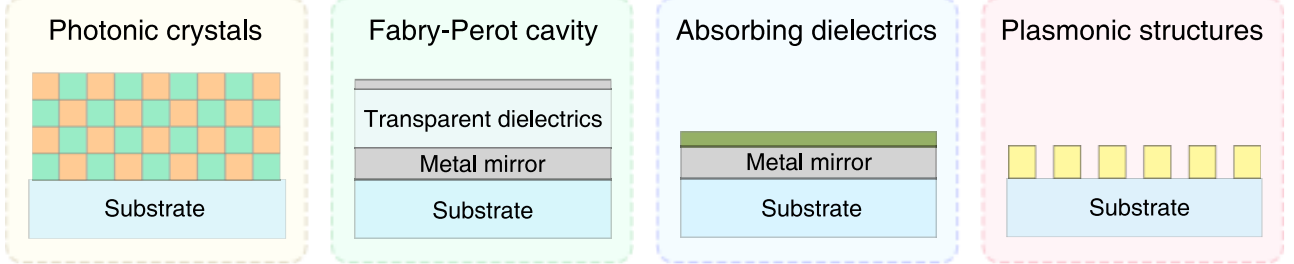

Figure 3.10 | Structural colour generation mechanisms. Four typical strategies are shown: photonic crystals with periodic arrangement of two materials with different permittivities, FabryPerot cavity with metal-insulator-metal structures, absorbing dielectric structure utilizing a lossy dielectric layer as colour filter, and plasmonic nanostructures to control light scattering.

Photonic crystal is a category of composites with periodic dielectric structure. In analogy with electronic structures of semiconductors, they are designed to form photonic band structures ${ }^{84}$. By precise arrangement of the permittivities and periodicities of the materials, it is possible to allow or forbid light transmission at certain wavelengths. Photonic crystals can be one-, two-, or three-dimensional, and made of layers, 
wires, or particles. Photonic crystals have been demonstrated for producing full-colour flat-panel displays ${ }^{85}$.

Fabry-Perot cavities are formed by sandwiching a transparent dielectric layer between a semi-transparent mirror and a highly reflecting mirror ${ }^{86}$. Structural colours are formed by wavelength-dependent constructive or destructive interference patterns between reflected light from the top and bottom mirrors. Variation in thickness of the dielectric spacer layer can be used to form reflectance peaks or dips at different wavelengths. This strategy has been commonly used for colour generation ${ }^{46,87-89}$.

Similar to Fabry-Perot cavities, ultrathin absorbing dielectric layers can also be used to form structural colours by interference or colour filter effect. For example, it has been shown that the optical reflectance and absorption of an ultrathin germanium layer was affected by its thickness ${ }^{90}$. Thus, a simple structure of germanium/gold can create thin film interference colours from blue to red with a relatively small angledependence ${ }^{90,91}$.

Plasmonics is the last approach we introduce here. As demonstrated in Section 3.2.2, the geometric factors of nanostructures can define their optical behavior. Tuning the shape, height, width, or pitch of plasmonic nanostructures enables colour variation in a relatively broad range and with high reflectance ${ }^{92}$. Plasmonic structural colour generation has been broadly used for high reflectivity colour generation by various fabrication methods ${ }^{93}$.

To achieve high resolution of images with multiple colours, advanced nanofabrication methods like electron-beam lithography and focused-ion-beam milling are typically needed ${ }^{93}$. For example, the thickness of dielectric layer in a Fabry-Perot cavity could be locally modulated by electron beam exposure ${ }^{89}$. Despite ultrahigh resolution, these methods remain challenging for scale-up production for commercialization. Recently, a laser-based method combined with imprinted nanostructures showed an alternative method for high-resolution colour production ${ }^{94}$.

\subsubsection{Combination with electrochromism}

Electrochromism is the phenomenon that the application of electrical bias can change the colour or transmission of a materia ${ }^{95}$. For conducting polymers, the electrochromism is related to their mixed ionic-electronic properties, where alternation of their redox state results in changes in electronic structure and electronic transition energies (Section 4.1.4). However, organic electrochromic materials provide quite limited possibility for colour tuning and are instead mostly used to tune overall transmission (between opaque and transparent). Such transmission tuning, however, can serve as a switching mechanism in combination with structural colour devices that provide different vivid colours (see Figure 3.11).

The configuration of tandem cells is the simplest strategy for such combination, where an electrochromic device is connected in series on top of a structural colour device (see Figure 3.11) ${ }^{46,88}$. However, the electrochromic material could also be directly 
integrated into a structural colour device. For example, electrochromic tungsten oxide or polymer layers could be employed as the spacing layer in a Fabry-Perot cavity and a gap plasmon device, respectively (Figure 3.11) ${ }^{96,97}$. Similarly, plasmonic structures could also be used as the working electrode in an electrochromic device to enhance its colour generation (Figure 3.11) ${ }^{98}$. In this thesis, we proposed a new and simple approach to form dynamic structural coloration using electrochromic PEDOT thin films on metal surfaces (Paper 4).
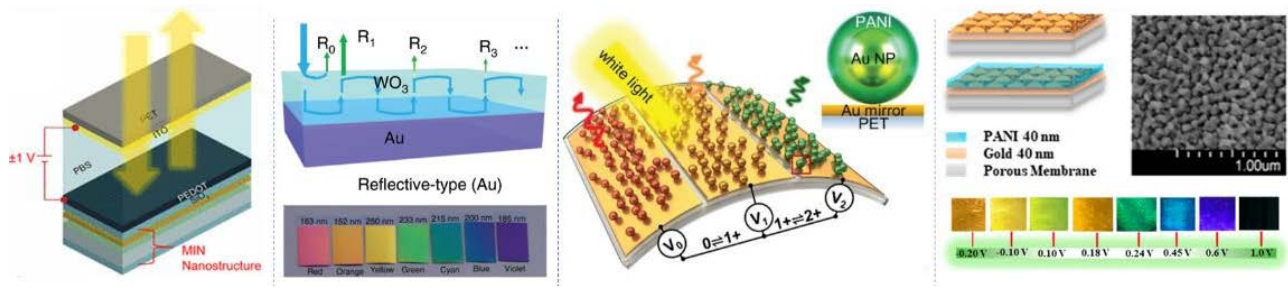

Figure 3.11 | Electrochromism and its hybrid devices with structural colours. The figures are reproduced from Reference 46, 96, 97, and 98 with permission from American Chemical Society, Springer Nature, and the American Association for the Advancement of Science.

\subsection{References}

Maier, S. A. Plasmonics: fundamentals and applications. (Springer Science \& Business Media, 2007).

2 Kittel, C. Introduction to solid state physics. ( John Wiley \& Sons, 1996).

3 Barnes, W. L., Dereux, A. \& Ebbesen, T. W. Surface plasmon subwavelength optics. Nature 424, 824-830 (2003).

$4 \quad$ Chen, S. et al. Conductive polymer nanoantennas for dynamic organic plasmonics. Nature Nanotechnology 15, 35-40 (2020).

Wokaun, A., Gordon, J. P. \& Liao, P. F. Radiation damping in surface-enhanced Raman scattering. Physical Review Letters 48, 957 (1982). Pt and Pd nanostructures. Nano letters 6, 833-838 (2006). ing, 2020). Han, Z. \& Bozhevolnyi, S. I. Radiation guiding with surface plasmon polaritons. Reports on Progress in Physics 76, 016402 (2012). Jackson, J. B. \& Halas, N. J. Surface-enhanced Raman scattering on tunable plasmonic nanoparticle substrates. Proceedings of the National Academy of Sciences 101, 17930-17935 (2004). Kumar, G. V. P. Plasmonic nano-architectures for surface
a review. Journal of Nanophotonics 6, 064503 (2012). Li, J.-F., Li, C.-Y. \& Aroca, R. F. Plasmon-enhanced fluorescence spectroscopy. Chemical Society Reviews 46, 3962-3979 (2017).

Höflich, K., Becker, M., Leuchs, G. \& Christiansen, S. Plasmonic dimer antennas for surface enhanced Raman scattering. Nanotechnology 23, 185303 (2012).

3 Schuller, J. A. et al. Plasmonics for extreme light concentration and manipulation. Nature materials 9, 193-204 (2010). 
Kawata, S., Inouye, Y. \& Verma, P. Plasmonics for near-field nano-imaging and superlensing. Nature photonics 3, 388-394 (2009).

$\mathrm{Li}$, K. et al. Balancing near-field enhancement, absorption, and scattering for effective antenna-reactor plasmonic photocatalysis. nano letters 17, 3710-3717 (2017).

Xia, Y., Xiong, Y., Lim, B. \& Skrabalak, S. E. Shape-controlled synthesis of metal nanocrystals: simple chemistry meets complex physics? Angewandte Chemie International Edition 48, 60-103 (2009).

Wiley, B. J. et al. Synthesis and optical properties of silver nanobars and nanorice. Nano letters 7, 1032-1036 (2007).

Curto, A. G. et al. Unidirectional emission of a quantum dot coupled to a nanoantenna. Science 329, 930-933 (2010).

Ni, X., Emani, N. K., Kildishev, A. V., Boltasseva, A. \& Shalaev, V. M. Broadband light bending with plasmonic nanoantennas. Science 335, 427-427 (2012).

1 Alu, A. \& Engheta, N. Tuning the scattering response of optical nanoantennas with nanocircuit loads. Nature photonics 2, 307-310 (2008).

2 Atwater, H. A. \& Polman, A. Plasmonics for improved photovoltaic devices. Nature Materials 6, 130-132 (2012).

3 Fusella, M. A. et al. Plasmonic enhancement of stability and brightness in organic lightemitting devices. Nature 585, 379-382 (2020).

4 Mayer, K. M. \& Hafner, J. H. Localized surface plasmon resonance sensors. Chemical reviews 111, 3828-3857 (2011).

5 Kedem, O., Vaskevich, A. \& Rubinstein, I. Critical issues in localized plasmon sensing. The Journal of Physical Chemistry C 118, 8227-8244 (2014).

7 Naik, G. V., Shalaev, V. M. \& Boltasseva, A. Alternative plasmonic materials: beyond gold and silver. Advanced Materials 25, 3264-3294 (2013).

Hirsch, L. R. et al. Nanoshell-mediated near-infrared thermal therapy of tumors under magnetic resonance guidance. Proceedings of the National Academy of Sciences 100 , 13549-13554 (2003).

A 1, 5790-5805 (2013).

Neumann, O. et al. Solar vapor generation enabled by nanoparticles. ACS nano 7, 4249 (2013).

Garnett, E. C. et al. Self-limited plasmonic welding of silver nanowire junctions. Nature Materials 11, 241-249 (2012). Mauser, K. W. et al. Resonant thermoelectric nanophotonics. Nature nanotechnology 12, 770 (2017).

Shiran Chaharsoughi, M. et al. Hybrid Plasmonic and Pyroelectric Harvesting of Light Fluctuations. Advanced Optical Materials 6, 1701051 (2018).

4 Tordera, D., Zhao, D., Volkov, A. V., Crispin, X. \& Jonsson, M. P. Thermoplasmonic semitransparent nanohole electrodes. Nano letters 17, 3145-3151 (2017).

5 Chaharsoughi, M. S. et al. Ultrasensitive electrolyte-assisted temperature sensor. npj Flexible Electronics 4, 1-7 (2020).

6 Shiran Chaharsoughi, M., Zhao, D., Crispin, X., Fabiano, S. \& Jonsson, M. P. Thermodiffusion-Assisted Pyroelectrics-Enabling Rapid and Stable Heat and Radiation Sensing. Advanced Functional Materials 29, 1900572 (2019). and technology. Nature nanotechnology 10, 25-34 (2015).

Reineck, P. et al. A solid-state plasmonic solar cell via metal nanoparticle self-assembly. Advanced Materials 24, 4750-4755 (2012). 
Shokri Kojori, H. et al. Plasmon field effect transistor for plasmon to electric conversion and amplification. Nano letters 16, 250-254 (2016).

Hervier, A., Renzas, J. R., Park, J. Y. \& Somorjai, G. A. Hydrogen oxidation-driven hot electron flow detected by catalytic nanodiodes. Nano letters 9, 3930-3933 (2009).

Zhou, L. et al. Quantifying hot carrier and thermal contributions in plasmonic photocatalysis. Science 362, 69-72 (2018).

Pelton, M., Aizpurua, J. \& Bryant, G. Metal-nanoparticle plasmonics. Laser \& Photonics Reviews 2, 136-159 (2008).

Lindquist, N. C., Nagpal, P., McPeak, K. M., Norris, D. J. \& Oh, S.-H. Engineering metallic nanostructures for plasmonics and nanophotonics. Reports on Progress in Physics 75, 036501 (2012).

Knight, M. W. et al. Aluminum for plasmonics. ACS nano 8, 834-840 (2014).

Nugroho, F. A. A. et al. Metal-polymer hybrid nanomaterials for plasmonic ultrafast hydrogen detection. Nature materials 18, 489-495 (2019).

6 Xiong, K. et al. Switchable plasmonic metasurfaces with high chromaticity containing only abundant metals. Nano Letters 17, 7033-7039 (2017).

7 Duan, X. \& Liu, N. Magnesium for dynamic nanoplasmonics. Accounts of Chemical Research 52, 1979-1989 (2019).

8 Duan, X., Kamin, S. \& Liu, N. Dynamic plasmonic colour display. Nature Communications 8, 1-9 (2017).

9 Boltasseva, A. \& Shalaev, V. M. All that glitters need not be gold. Science 347, 13081310 (2015).

West, P. R. et al. Searching for better plasmonic materials. Laser \& Photonics Reviews 4, 795-808 (2010).

Boltasseva, A. \& Atwater, H. A. Low-loss plasmonic metamaterials. Science 331, 290291 (2011).

Li, S. Q. et al. Infrared plasmonics with indium-tin-oxide nanorod arrays. ACS nano 5, 9161-9170 (2011).

3 Riley, C. T. et al. Plasmonic tuning of aluminum doped zinc oxide nanostructures by atomic layer deposition. physica status solidi (RRL)-Rapid Research Letters 8, 948952 (2014).

4 Luther, J. M., Jain, P. K., Ewers, T. \& Alivisatos, A. P. Localized surface plasmon resonances arising from free carriers in doped quantum dots. Nature materials 10, 361366 (2011).

5 Llorente, V. B. et al. Electrochemical tuning of localized surface plasmon resonance in copper chalcogenide nanocrystals. The Journal of Physical Chemistry C 121, 1824418253 (2017).

$6 \mathrm{Li}, \mathrm{W}$. et al. Refractory plasmonics with titanium nitride: broadband metamaterial absorber. Advanced Materials 26, 7959-7965 (2014).

7 Rodrigo, D. et al. Mid-infrared plasmonic biosensing with graphene. Science 349, 165168 (2015).

Dutta, A. et al. Strontium Niobate for Near-Infrared Plasmonics. Advanced Optical Materials 7, 1900401 (2019).

9 Grigorenko, A. N., Polini, M. \& Novoselov, K. S. Graphene plasmonics. Nature photonics 6, 749-758 (2012).

0 Garcia de Abajo, F. J. Graphene plasmonics: challenges and opportunities. Acs Photonics 1, 135-152 (2014).

1 Fei, Z. et al. Gate-tuning of graphene plasmons revealed by infrared nano-imaging. Nature 487, 82-85 (2012).

2 Chen, J. et al. Optical nano-imaging of gate-tunable graphene plasmons. Nature 487, 77-81 (2012). 
63 Fang, Z. et al. Active tunable absorption enhancement with graphene nanodisk arrays Nano letters 14, 299-304 (2014).

64 Fang, Z. et al. Gated tunability and hybridization of localized plasmons in nanostructured graphene. ACS Nano 7, 2388-2395 (2013). Gopalan, K. K. et al. Scalable and Tunable Periodic Graphene Nanohole Arrays for Mid-Infrared Plasmonics. Nano Letters 18, 5913-5918 (2018).

Matsui, T., Vardeny, Z. V., Agrawal, A., Nahata, A. \& Menon, R. Resonantly-enhanced transmission through a periodic array of subwavelength apertures in heavily-doped conducting polymer films. Applied physics letters 88, 071101 (2006).

Chen, S. et al. On the anomalous optical conductivity dispersion of electrically conducting polymers: ultra-wide spectral range ellipsometry combined with a Drude-Lorentz model. Journal of Materials Chemistry C 7, 4350-4362 (2019).

Yan, F., Parrott, E. P. J., Ung, B. S. Y. \& Pickwell-MacPherson, E. Solvent doping of PEDOT/PSS: effect on terahertz optoelectronic properties and utilization in terahertz devices. The Journal of Physical Chemistry C 119, 6813-6818 (2015).

Manjavacas, A. et al. Tunable molecular plasmons in polycyclic aromatic hydrocarbons. ACS nano 7, 3635-3643 (2013).

Stec, G. J., Lauchner, A., Cui, Y., Nordlander, P. \& Halas, N. J. Multicolor electrochromic devices based on molecular plasmonics. ACS nano 11, 3254-3261 (2017).

Lauchner, A. et al. Molecular plasmonics. Nano letters 15, 6208-6214 (2015). Gu, L., Livenere, J., Zhu, G., Narimanov, E. E. \& Noginov, M. A. Quest for organic plasmonics. Applied Physics Letters 103, 021104 (2013).

Gentile, M. J., Núñez-Sánchez, S. \& Barnes, W. L. Optical field-enhancement and subwavelength field-confinement using excitonic nanostructures. Nano letters 14, 2339 2344 (2014).

Woo, B. H. et al. Dispersion control of excitonic thin films for tailored superabsorption in the visible region. ACS Photonics 4, 1138-1145 (2017).

Yilmaz, M. et al. Nanostructured organic semiconductor films for molecular detection with surface-enhanced Raman spectroscopy. Nature Materials 16, 918-924 (2017). Shaltout, A. M., Shalaev, V. M. \& Brongersm
active metasurfaces. Science 364 (2019).

able substrate. Nano letters 16, 2818-2823 (2016).

8 Tseng, M. L. et al. Two-dimensional active tuning of an aluminum plasmonic array for full-spectrum response. Nano letters 17, 6034-6039 (2017).

Peng, J. et al. Scalable electrochromic nanopixels using plasmonics. Science advances 5, eaaw2205 (2019).

Nassau, K. Color for science, art and technology. (Elsevier, 1997).

Hunt, R. W. G. \& Pointer, M. R. Measuring colour. (John Wiley \& Sons, 2011).

Ohno, Y. CIE fundamentals for color measurements. NIP \& Digital Fabrication Conference 2000, 540-545.

Tkalcic, M. \& Tasic, J. F. Colour spaces: perceptual, historical and applicational background. Vol. 1 (IEEE, 2003). munications 102, 165-173 (1997).

Arsenault, A. C., Puzzo, D. P., Manners, I. \& Ozin, G. A. Photonic-crystal full-colour displays. Nature Photonics 1, 468-472 (2007).

Kasap, S. O. Optoelectronics and photonics. (Prentice Hall, 2001).

Rossi, S. \& Jonsson, M. P. Highly reflective optical nanocavities for structural coloration by combining broadband absorber and Fabry-Pérot effects. Journal of Optics 23, 015001 (2020). 
88 Xiong, K. et al. Plasmonic metasurfaces with conjugated polymers for flexible electronic paper in color. Advanced Materials 28, 9956-9960 (2016).

89 Daqiqeh Rezaei, S., Ho, J., Wang, T., Ramakrishna, S. \& Yang, J. K. W. Direct Color Printing with an Electron Beam. Nano Letters (2020).

90 Kats, M. A., Blanchard, R., Genevet, P. \& Capasso, F. Nanometre optical coatings based on strong interference effects in highly absorbing media. Nature materials $\mathbf{1 2}$, 20-24 (2013).

91 Kats, M. A. et al. Ultra-thin perfect absorber employing a tunable phase change material. Applied Physics Letters 101, 221101 (2012).

92 Shao, L., Zhuo, X. \& Wang, J. Advanced plasmonic materials for dynamic color display. Advanced Materials 30, 1704338 (2018).

93 Kristensen, A. et al. Plasmonic colour generation. Nature Reviews Materials 2, 1-14 (2016).

94 Zhu, X., Vannahme, C., Højlund-Nielsen, E., Mortensen, N. A. \& Kristensen, A. Plasmonic colour laser printing. Nature nanotechnology 11, 325-329 (2016).

95 Monk, P. M. S., Mortimer, R. J. \& Rosseinsky, D. R. Electrochromism: fundamentals and applications. (John Wiley \& Sons, 2008).

96 Wang, Z. et al. Towards full-colour tunability of inorganic electrochromic devices using ultracompact fabry-perot nanocavities. Nature communications 11, 1-9 (2020).

97 Peng, J. et al. Fully Printed Flexible Plasmonic Metafilms with Directional Color Dynamics. Advanced Science, 2002419 (2020).

98 Shahabuddin, M., McDowell, T., Bonner, C. E. \& Noginova, N. Enhancement of electrochromic polymer switching in plasmonic nanostructured environment. ACS Applied Nano Materials 2, 1713-1719 (2019). 


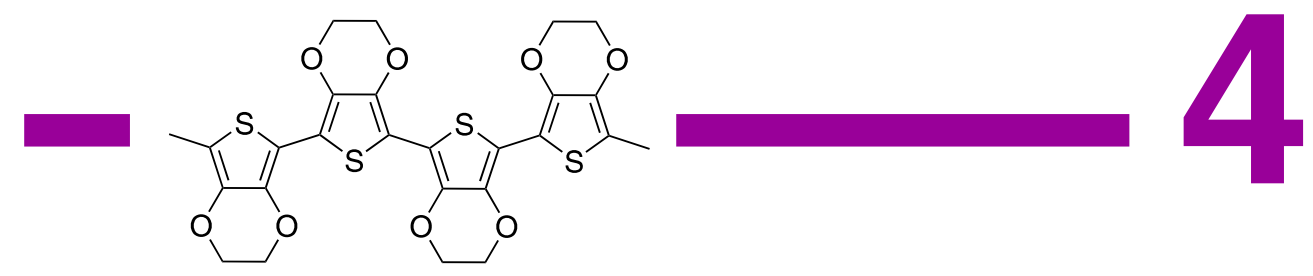

\section{Conducting Polymers}

Conducting polymers, especially poly(3,4-ethylenedioxythiophene) or PEDOT, are the materials in focus for this study. In this chapter, we start with a brief review of the basic properties of conducting polymers, from electronic structures to optical and electrical properties. We choose PEDOT as the model system to discuss the charge transport mechanisms of conducting polymers. We finish the chapter with a concise introduction of typical optical models for conducting polymers, which is relevant to the ellipsometry studies included in this thesis.

\section{Contents}

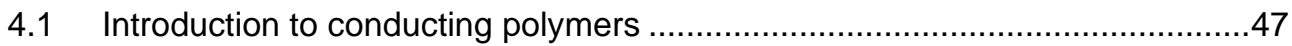

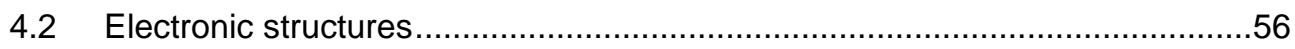

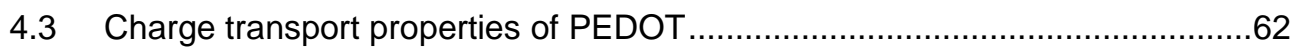

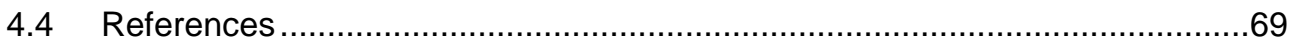

\subsection{Introduction to conducting polymers}

The word, polymer, was first introduced in 1833 by the Swedish chemist Jöns Jakob Berzelius, who was born in Väversunda ( $60 \mathrm{~km}$ from Linköping), although the definition was totally different from the meaning (proposed by Hermann Staudinger in 1920) we used today ${ }^{1}$. We are able to understand its meaning straightforwardly by dividing the 
word into two parts, where "poly" stands for multiple, while "mer" represents single unit or part. Polymers share two characteristic features: high molecular mass and high number of repeat units.

\subsubsection{The emergence of conducting polymers}

Most polymers are organic materials with backbones mostly made of carbon atoms, although inorganic monomers including $\mathrm{SN}$ and $\mathrm{SiN}$ can also be polymerized ${ }^{2}$. In this thesis, we limit our focus to organic polymers with only carbon (or sulfur) as the basic element in the backbones. The monomers in a polymer are connected via covalent bonds to form linear, branched, or cross-linked structurers. A typical covalent bond for such connection is single carbon-carbon bond (or $\sigma$ bond), where two electrons are shared by two carbon atoms and strongly localized in the area between the two atoms showing poor mobility. Thus, most polymers discovered in nature or synthesized by humans are insulators (see Figure 4.1).

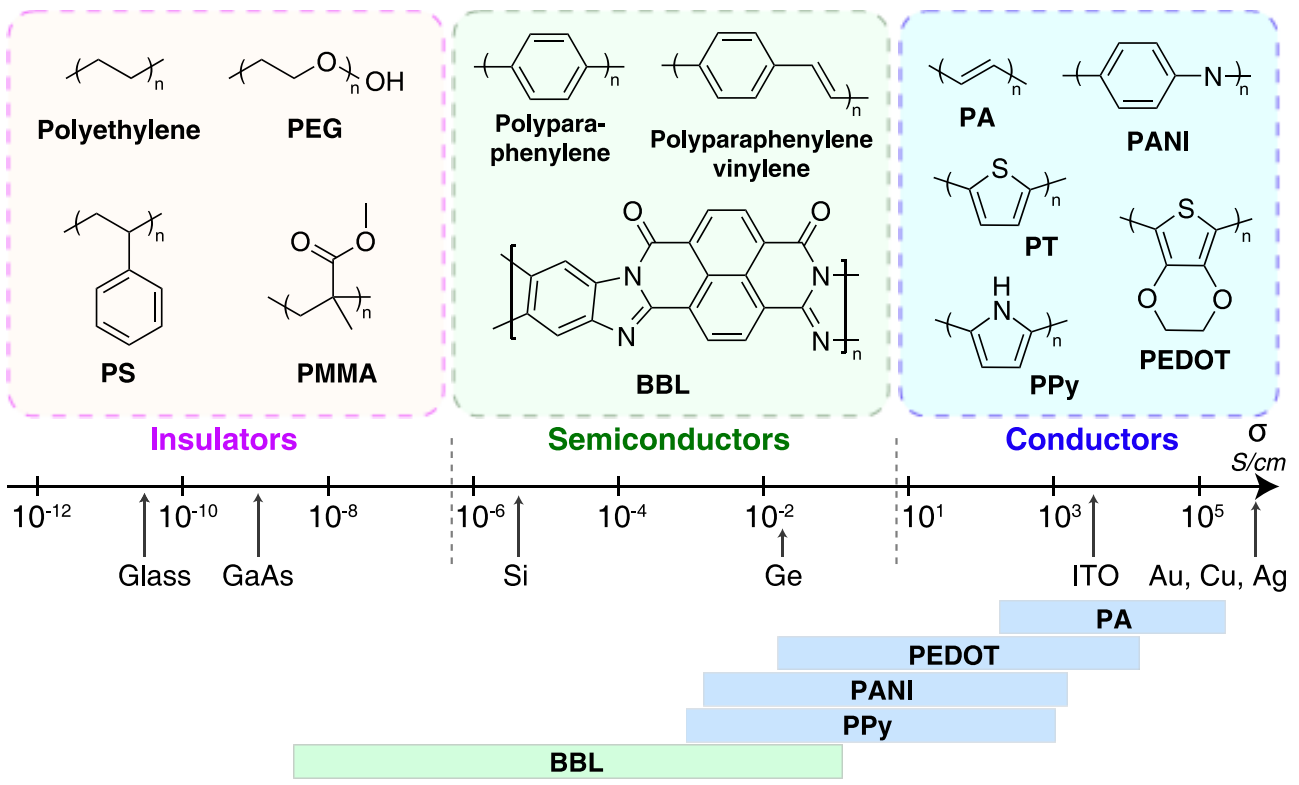

Figure 4.1 | Classification of polymers and their electrical conductivities. Based on their electrical conductivities, polymers can be divided into three classes: insulators, semiconductors, and conductors. By doping process, the conductivity of (semi-)conducting polymers can vary in a large range. Typical examples are listed for each category.

To generate mobile electrons in polymer systems, a possible strategy is to employ double carbon-carbon bonds (or $\sigma$ and $\pi$ bonds). Due to lateral overlapping of atomic orbitals, $\pi$ electrons are delocalized. By arranging the polymer backbone with alternating single and double carbon-carbon bonds, we are able to synthesize conjugated polymers with semiconducting properties. It was also found that introduction of 
(hetero)aromatic rings in the backbone can further improve semiconducting properties, profoundly influencing the design of conjugated polymers ${ }^{3}$. The most used conjugated polymers nowadays, such as polythiophene (PT), all share this (hetero)aromatic ring feature (see Figure 4.1).

Although the electrical properties of polymers can be improved with conjugation, their applications are limited due to rather poor electrical conductivity. It remained true that polymers could hardly conduct electricity, until the revolutionary discovery of intrinsically conducting polymers in 1977 by Alan Jay Heeger, Alan MacDiarmid, and Hideki Shirakawa ${ }^{4,5}$. Their pioneering work on polyacetylene (PA) doped by electron- and hole-donating chemicals, recognized as the first conducting polymer, was highlighted by the awarding of the Nobel Chemistry Prize in 2000 (Figure 4.2) ${ }^{6-8}$. Despite this, the earliest findings on conducting polymers can be traced back to 1960s, when Donald Weiss at the University of Adelaide, Australia, successfully obtained polypyrrole (PPy) powders ${ }^{9}$. They prepared PPy by heating tetraiodopyrrole in a flask with nitrogen flow, which maintained an inert environment and transferred generated iodine vapour away from the reaction. Interestingly, they found that the obtained PPy materials showed resistivity of $11-200 \mathrm{Ohm} \cdot \mathrm{cm}$ (or conductivity of $0.005-0.1 \mathrm{~S} / \mathrm{cm}$ ) and this value could be enhanced if an additional adsorbed iodine removal process was utilized. The origin of its conductivity is currently believed as a result of iodine doping although it was not fully understood at that time. Similarly, in 1966, Marcel Jozefowicz and Rene Buvet at ESPCI ParisTech, France, reported their findings on oxidative polymerization of polyaniline $(\mathrm{PANI})^{9}$. They found that the conductivity of PANI had a strong dependence on the $\mathrm{pH}$ value of precursor solution and the value could reach $30 \mathrm{~S} / \mathrm{cm}$ when the $\mathrm{pH}$ value of precursor solution is minimized. All above studies were based on polymers in the form of powders or pellets, instead of thin films, making them less convenient for the electrical property study and attracting limited attention across the whole community.

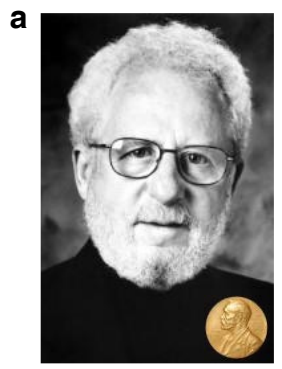

Alan Jay Heeger

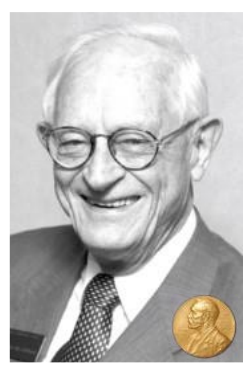

Alan MacDiarmid

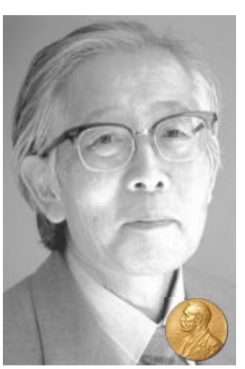

Hideki Shirakawa

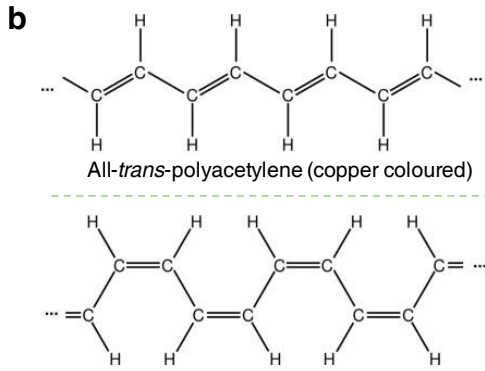

All-cis-polyacetylene (silver coloured)

Figure 4.2 | Nobel Prize Chemistry in 2000. a, Laureates of the Nobel Prize: Alan Jay Heeger, Alan MacDiarmid, and Hideki Shirakawa. b, Polyacetylene in all-trans or all-cis form. The photos of the Nobel Prize laureates were collected from the Nobel Prize official website ${ }^{10}$.

The first conducting polymer in the thin film form was demonstrated in 1967 by Hideki Shirakawa and his visiting Korean student Hyung Chick Pyun ${ }^{11}$. They obtained the film 
by accident when they mistakenly used a much higher catalyst concentration compared to the standard recipe of $\mathrm{PA}^{9}$. Instead of powders, the obtained PA films exhibited coppery or silvery colours depending on cis- or trans-form of PA. The conductivity was found to be $10^{-9}$ to $10^{-8} \mathrm{~S} / \mathrm{cm}$ for all-trans-form and $10^{-5}$ to $10^{-4} \mathrm{~S} / \mathrm{cm}$ for all-cis-form (Figure 4.2b), much lower compared to PPy and PANI. At that time, Alan MacDiarmid and Alan Jay Heeger were focusing on the poly(sulfurnitride) or $(\mathrm{SN})_{x}$, which shares a similar chemical structure as PA but showed a higher conductivity of $1200-3700 \mathrm{~S} / \mathrm{cm}^{9}$. Interestingly, they found that the electrical conductivity of poly(sulfurnitride) could be further enhanced by bromine treatment.

The collaboration between the three scientists started in 1975, when Shirakawa got a visiting scholarship for one year stay in US. Their initial work was primarily purification of PA, since they thought high purity of films should enhance the electrical properties. They soon realized the fact that the reduction of impurity concentration also decreased the electrical conductivity. Inspired by their previous studies on poly(sulfurnitride), Heeger and MacDiarmid attempted to treat the PA film with bromine, and found that the conductivity increased from $10^{-5}$ to $0.5 \mathrm{~S} / \mathrm{cm}$ within only 10 minutes. The value could be further pushed to $160 \mathrm{~S} / \mathrm{cm}$ by iodine and above $560 \mathrm{~S} / \mathrm{cm}$ by AsF 5 treatment $^{4,5}$. Interestingly, instead of electron-accepting chemicals above, treatment with electron-donating chemicals like sodium could also increase the conductivity to a maximum of $200 \mathrm{~S} / \mathrm{cm}^{12}$, making PA unique compared to other conducting polymers. The process of electron donating or accepting is similar to the doping in inorganic semiconductors and is defined as n-doping (reduction) or p-doping (oxidation) of conducting polymers.

With great efforts by follow-up researches, PA films were able to reach a high conductivity of $2 \times 10^{5} \mathrm{~S} / \mathrm{cm}$, approaching that of copper or silver ${ }^{13}$. However, an issue for the fabrication and processing of PA is that oxygen could easily degrade the film and all experiments had to be done in controlled environment ${ }^{14}$. For practical applications, researchers had to seek for alternatives with high stability in air. Currently, hundreds of conducting polymers have been demonstrated but only several of them are still being used, including PPy, PANI, and PEDOT. These polymers could reach electrical conductivity of around $1000 \mathrm{~S} / \mathrm{cm}$ when they are doped with suitable counterions ${ }^{15}$. The relatively low conductivity, compared to metals, might be due to various reasons, such as the poor crystallinity of the films induced by the size of monomers.

Certain strategies have been proposed for conductivity enhancement, by introducing novel thin film processing methods (e.g., stretchable substrates ${ }^{16}$, solution-shearing ${ }^{17}$, and aggregation control $^{18}$ ), fabrication techniques (e.g., ultrathin layer deposition ${ }^{19,20}$, and nanowires ${ }^{21}$ ), and specific doping protocols (e.g., co-solvent engineering ${ }^{22-24}$, and acid treatment $\left.{ }^{25,26}\right)$. Up to now, the electrical conductivity record for non-PA conducting polymer is $2 \times 10^{5} \mathrm{~S} / \mathrm{cm}$ of $\mathrm{C}_{12}$-PBTTT by Martin Brinkmann et al. ${ }^{27}$

\subsubsection{Charge carriers of conducting polymers}


Before we further introduce details on doping in polymers, we need to review the basics of charge carriers in the conducting polymers, namely, polarons, bipolarons, and solitons. When a $p$-type dopant is added into an electrically neutral system of $p$ type polymer (e.g., polythiophene or PT), one electron is removed from the polymer and a positive charge forms on the chain (Figure 4.3). The charge, or radical cation, is localized and attracted by the ionized dopants, or counterions due to Coulomb attraction. Such process causes distortion of the segment deviating from its equilibrium geometry in neutral state. For PT, the local thiophene ring can change from aromatic form (in neutral state) to quinoid form (in doped state). The charge together with the local distortion of polymer chain is defined as a polaron ${ }^{28}$. Polarons serve as the charge carriers for the electronic conduction, and their motion along the backbone has to be accompanied by counterions, which typically have a low mobility.

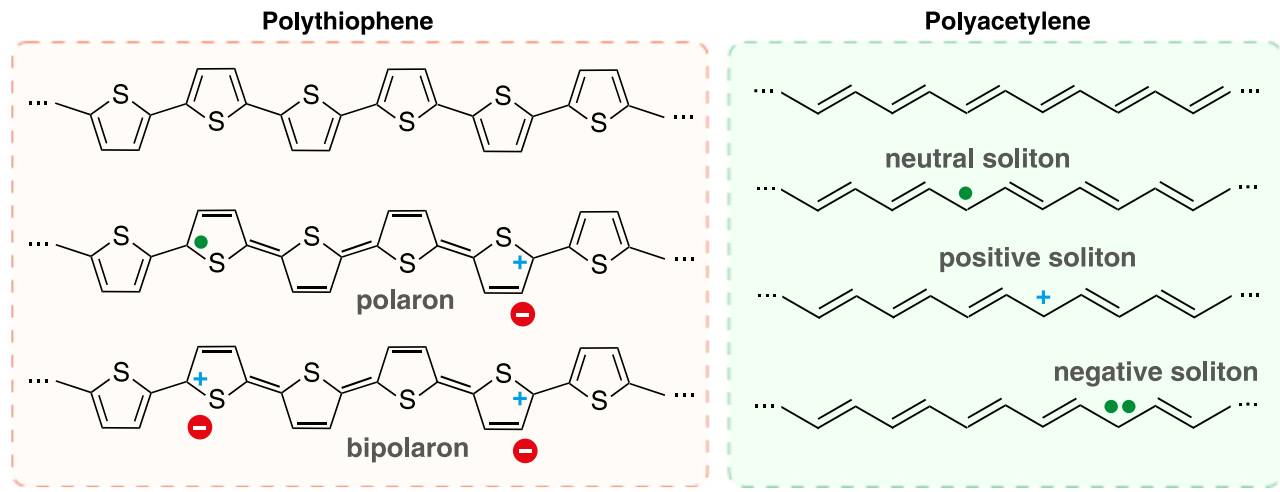

Figure 4.3 | Basic charge carriers in conducting polymers. Polaron and bipolaron are shown in the left column using PT as the example while soliton is exhibit in the right column using PA as the example. The doping changes thiophene ring in PT from aromatic form (neutral state) to quinoid form (with polarons or bipolarons).

Bipolaron can form when a second electron is removed from the segment, resulting in two charges on the segment (Figure 4.3). A bipolaron is a combination of two positive (negative) charges and their local chain distortion. The concept of bipolaron is similar to the Cooper pair in superconductors ${ }^{29}$, where two electrons with identical negative charge are attracted to each other via lattice deformation. Bipolarons are more thermodynamically stable for polymers with high doping concentration due to the fact that its formation energy is smaller than that of two polarons ${ }^{28}$. It should be highlighted that the charge carried by a bipolaron is $\pm 2 e$, and the spin is 0 , compared to charge of $\pm e$ and spin of $1 / 2$ by a polaron. Therefore, electron paramagnetic resonance is widely used to distinguish the density of polarons and bipolarons in conducting polymers ${ }^{30,31}$.

A soliton, or solitary wave defect, is a specific type of charge carriers in PA when the polymer backbone has odd number of carbon atoms ${ }^{6}$. Solitons could be generated during the thermal isomerization of a PA chain between its cis and trans forms ${ }^{6}$. A soliton can be viewed as a topological kink in the electron-lattice system or a boundary 
separating two phases with opposite bond alternations ${ }^{32}$. Solitons can be neutral (charge of 0 and spin of $1 / 2$ ) or carry positive (charge of $+e$ and spin of 0 ) or negative charge (charge of $-e$ and spin of 0 ) formed by oxidation or reduction reactions. It should be noted that the movement of solitons cause negligible deformation of PA chain since cis and trans forms of PA share the same ground state energy (degenerated) ${ }^{28}$.

\subsubsection{Doping in conducting polymers}

The concept of doping in conducting polymers is proposed as an analogy to the doping in inorganic semiconductors, although there are drastic differences. Firstly, the doping in conducting polymers is achieved by inserting ions, small molecules, or polyelectrolytes between polymer chains, instead of replacing atoms in the chains ${ }^{33}$. Secondly, charge carriers in conducting polymers are generated via reduction or oxidation reactions with the involvement of charge transfer, instead of releasing surplus carriers in inorganics. Lastly, the dopant concentration in conducting polymers is usually much larger (can even reach $200 \%{ }^{34}$ ) than in inorganic semiconductors (normally smaller than $0.01 \%{ }^{33}$ ). It should also be noted that doping in conducting polymers is a generalized concept that does not need to be associated with introduction of charge carriers ${ }^{35}$. For example, adding polar solvents or plasticizer molecules into a polymer system leads to changes in the film morphology or microstructure, which can also result in an enhancement of electrical conductivities ${ }^{35}$. Although these additives do not introduce additional charge carriers, we still regard them as dopants (nanostructuring agents) for polymers. Thus, two types of doping processes exist in conducting polymers: one is primary (redox state) doping, while the other is secondary (microstructural or morphological) doping.

Primary doping is achieved by introducing dopants either by oxidization to create a positive charge ( $\mathrm{p}$-type) or reduction to generate a negative charge (n-type) on the conducting polymer chain. Commonly used $p$-type and n-type dopants can be small ions, organic molecules, ionic liquids, and polyelectrolytes (see Figure 4.4). Dopants can be introduced into polymers as counterions by different methods, including direct mixing of two materials in a solvent, chemical vapour treatment, solvent treatment, and bi-layer deposition followed by a thermal annealing step ${ }^{36}$. The commonly used methods are in-situ chemical doping during the polymer synthesis and electrochemical doping, which will be introduced in Section 4.1.4).

For in-situ doping, the dopants (e.g., $\mathrm{FeCl}_{3}$ or $\mathrm{Fe}$ (p-toluenesulfonate) $)_{3}$ ) can act as both precursors or oxidants to initiate the polymerization reaction and as counterions to guarantee electroneutrality of the system ${ }^{37}$. The commercialized PEDOT products in the market are already doped with a polyelectrolyte, poly(4-styrene sulfonate) or PSS, dissolved in water. The conductivity of the product can be further enhanced by adding a co-solvent like ethylene glycol (EG) or dimethyl sulfoxide (DMSO) ${ }^{35}$. Such co-solvent will not introduce charges to the polymer but optimize the morphology of the film as a nanostructuring agent and remove excessive insulating PSS components in the film, improving the conducting polymer networks for efficient carrier transport in the film ${ }^{23,38}$. 
In addition to external dopants, some conducting polymers can be doped by themselves. The process is known as self-doping. In self-doped polymers, the monomer is modified by introducing a side-chain containing dopant ${ }^{39}$. One example is poly $(4-(2,3-$ dihydrothieno[3,4-b]-[1,4]dioxin-2-yl-methoxy)-1-butanesulfonic acid, sodium salt) ${ }^{40}$ or PEDOT-S, with high electrical conductivity of $30 \mathrm{~S} / \mathrm{cm}^{39}$. However, self-doping strongly relies on the synthesis of specific monomers and is inconvenient compared to external dopants with various choices.

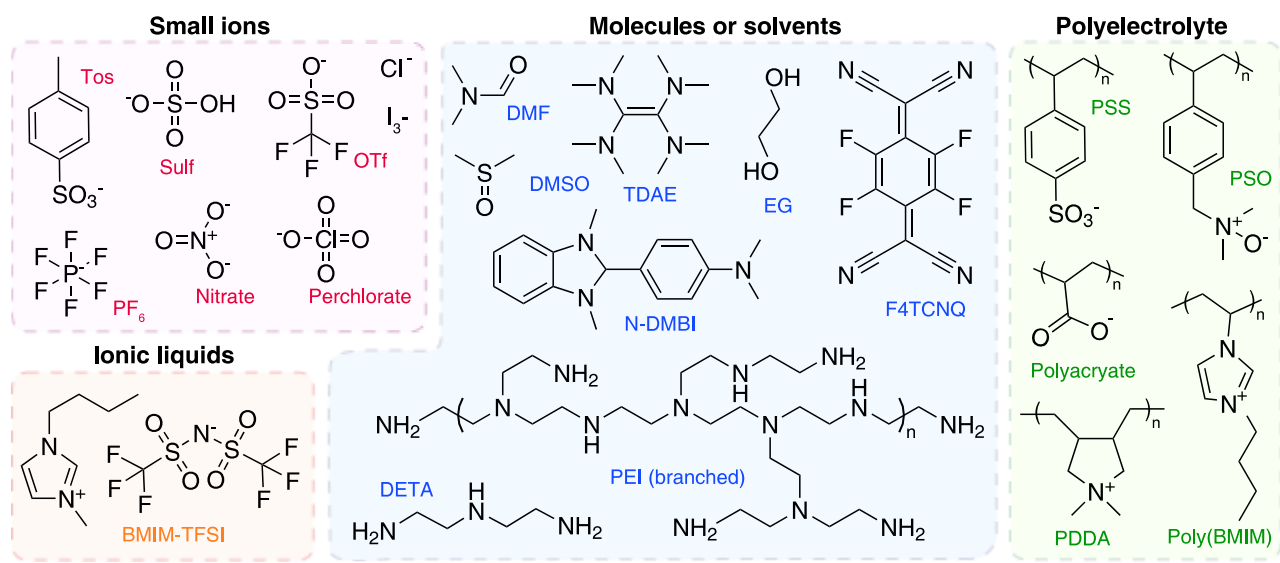

Figure 4.4 | Commonly used dopants or counterions for conducting polymers. Four types are summarized: small ions, ionic liquids, molecules (or polymers), and polyelectrolytes.

In inorganic semiconductors, the doping efficiency is evaluated by the concentration or density of dopants, while in conducting polymers, these parameters are less useful for multiple reasons. A large number of dopants may not contribute to charge carrier generation, but reside in the polymer matrix neutrally, e.g., excessive PSS in PEDOT films ${ }^{37,41}$ (which is similar to incompletely ionized dopants in inorganic semiconductors). Instead, the oxidation level, which is defined as the ratio of active dopants (carrying charges) to monomers, is the widely used parameter in conducting polymers. Methods including X-ray photoelectron spectroscopy (XPS) and Raman spectro-scopy can be used to measure the oxidation level of conducting polymers ${ }^{24,42}$. The oxidation level provides insight into the charge carrier density, although other parameters including film density, defects, and morphology also play important roles. Hall effect measurements ${ }^{43}$, optical measurements ${ }^{44}$ (ultrawide spectral range reflectivity or spectroscopic ellipsometry), and electrochemical methods (e.g., chronocoulometry $)^{45}$ are possible approaches to determine carrier density in conducting polymer films.

\subsubsection{Mixed ionic-electronic conductors}

The counterions used in conducting polymers are mainly responsible for the balance of charges on the conducting polymer, but can also be available for other applications when exposed to electrolytes or humidity. This is a critical difference between organic 
and inorganic conductors. The capability of ionic transport in conducting polymers enables numerous applications that are impossible for inorganics ${ }^{46}$. Ions can be injected into a conducting polymer film via an electrolyte (either in solution or solid state made of ionic liquid in a polymer matrix) and propagate in the film along the applied electric field direction (for cations) with a mobility comparable to that of ions in water ${ }^{46}$. Such property also makes it possible for conducting polymer to be doped in an electrochemical way (Figure 4.5). Electrochemical doping can be used to alter the electrical, optical, or mechanical properties of conducting polymers in a more precise manner than using chemical doping, and is promising for practical devices ranging from electrochemical transistors, electrochromic displays, and electrochemical actuators $^{47-49}$.
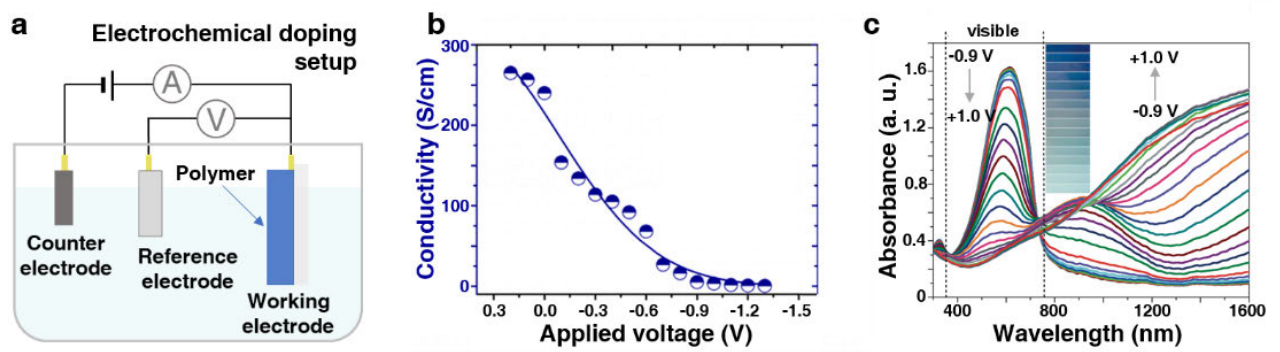

Figure 4.5 | Electrochemical doping of conducting polymers. a, The properties of PEDOT can be precisely controlled using an electrochemical cell with three electrodes. b. Electrical conductivity of the conducting polymer can be electrochemically altered in a large range (reproduced from Reference 50 with permission from American Chemical Society). c, Colour (inset) and optical absorbance of the polymer film can be controlled by different electrochemical bias (reprinted from Reference 51 with permission from John Wiley and Sons).

In the dry state of a conducting polymer film, the ionic transport is largely hindered, while an increase in humidity can rapidly recover the ionic transport properties ${ }^{52}$. The polymer films are soft and permeable for ions and solvent molecules (e.g., water) which can easily diffuse into the polymer film leading to microstructural changes, such as swelling. Hydration of PEDOT therefore offers ion-transport pathways that are heavily influenced by the domain size and composition at the mesoscale of the polymer ${ }^{53}$. Despite a limited understanding on the ionic transport, some studies indicated that ion-transport pathways might be formed in counterion-rich regions, like in the case of PEDOT:PSS films 46,53 . Secondary doping of PEDOT:PSS increases the electrical conductivity of the film but might reduce its ionic mobility ${ }^{53}$. Hydrophilicity is another important factor affecting the ionic transport in polymer films and by incorporating hydrophilic additives like cellulose or gelatin ${ }^{54}$, the ionic mobility can be improved, despite a reduction in the electronic conductivity. A trade-off often exists between ionic and electronic properties and a detailed optimization for certain applications would be necessary. 
Recently, in operando $\mathrm{x}$-ray scattering results of conducting polymers revealed the time-resolved evolution of microstructures at different electrochemical redox states ${ }^{55}$. It showed that the lamella stacking of the polymer chains experiences both expansion and relaxation during the doping process, with asymmetric rates ${ }^{55}$. Another interesting study on the coupling between ionic and electronic properties is the self-healing phenomenon of PEDOT:PSS films, where water or humidity could induce counterion diffusion transport towards scratched areas, reconstructing the thin film and recovering its initial electrical properties ${ }^{52}$.

\subsubsection{Applications and future potentials of conducting polymers}

Numerous achievements have been made in the synthesis, property optimization, and commercialization of conducting polymers since their discovery. The initial idea of utilizing conducting polymers was just an alternative to metals for electrical cables and batteries to reduce weights and expenses in automobiles and airplanes. Even if the conductivity is not high, they could find applications like in antistatic or electromagnetic radiation shielding coatings, both of which are the most successful products of conducting polymers with market value approaching 1 billion US dollars in $2018 .^{3}$

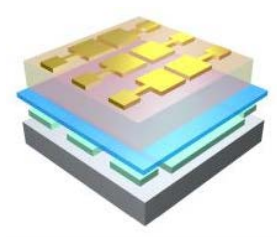

Organic solar cells or light-emitting diodes

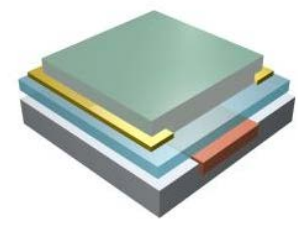

Organic thin film transistors

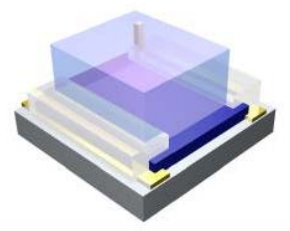

Organic electrochemical transistors

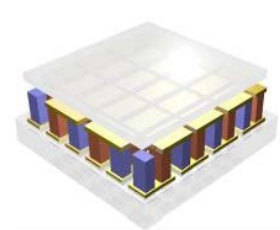

Organic thermoelectric generators

Figure 4.6 | Applications of conducting polymers. Four typical devices are shown: organic optoelectronic devices (solar cells or light-emitting diodes), organic thin film transistors, organic electrochemical transistors, and organic thermoelectric generators.

Polymers with conducting or semiconducting properties are expected to be useful for optoelectronic, semiconducting, electromechanical, and electrochemical devices (see Figure 4.6) ${ }^{6}$. Some promising directions are their uses as transparent electrodes, hole or electron transporting layers, and active layers (donors or acceptors) in organic and perovskite solar cells ${ }^{56}$. Although a gap still exists between organic and silicon-based solar cells regarding device efficiency and operational stability, the rapid development of (semi-)conducting polymers is making efforts to remove the technology barriers towards practical devices ${ }^{57}$. Another potential application is organic thin film transistors, where the most used polymeric materials is poly(3-hexylthiophene) ${ }^{58}$. Compared to their inorganic counterparts, organic transistors are aimed for new applications like electronic papers, radiofrequency ID tags, and flexible displays, due to its upper limit of switching speed (conducting polymers have limitation in charge carrier mobility) ${ }^{59}$. The main advantage of polymers over inorganics used for transistors is their excellent mechanical properties (e.g., bendable, stretchable, and flexible) ${ }^{3}$. 
Despite many publications stating that the processing costs of conducting polymers are low and compatible with large-scale production, the price of most commercialized products is still high. For example, PEDOT:PSS can be purchased for 300-600 US dollars per kilogram (more expensive than most metals); and poly(3-hexylthiophene) has a price in the range between 1000 and 2000 US dollars per kilogram, making it less attractive compared to the low cost of amorphous silicon for thin film electronics ${ }^{3}$.

Recently, utilizing the mixed ionic-electronic properties of conducting polymers has become popular because it offers new opportunities in applications like electronic skin, the internet-of-things platforms, implantable bioelectronics, and neuromorphic computing devices ${ }^{60-62}$. With additional ionic properties, conducting polymers can be used for batteries, supercapacitors and thermoelectric generators ${ }^{63,64}$. New devices, including organic electrochemical transistors (OECT) with high transconductance ${ }^{48}$, especially those based on PEDOT:PSS films, received lots of attention and exhibited excellent performances for bio-applications ${ }^{48}$. Achievements of OECTs have been made within the past five years, including recording of electrophysiological signals ${ }^{65}$, stimulating electrical activity of the nervous systems ${ }^{66,67}$, regulating plant growth and physiology ${ }^{68}$, and controlling drug delivery to targets ${ }^{69}$. The successful fabrication of OECTs via lowcost printing techniques, which is impossible for most inorganic materials, enables large-scale integrated circuits based on OECTs with high yields, making it possible for mixed ionic-electronic devices to obtain more complicated and practical functions ${ }^{70,71}$. In addition, conducting polymers in the form of aerogels or hydrogels, instead of thin films, showed unique properties for bio-applications ${ }^{72-74}$. We believe that conducting polymers could largely accelerate the commercialization of bioelectronic devices targeting human health monitoring, point-of-care diagnostics, and precise drug delivery for medical treatments ${ }^{60}$.

Fundamental problems of conducting polymers still remain unsolved despite a huge progress in the synthesis and applications of high-performance polymers. For example, the charge transport properties, including the role of conjugation length, interchain transport, and crystallinity and morphology, are still not completely clear. The issues of degradation initiated by humidity, oxygen, heat, or light, for some polymers remain incompletely solved and many polymers could only be processed under inert gas in a glove box. The rapid development of new technologies, such as machine learning with big data and high-speed computation approaches (quantum computers) might enable possibilities to solve these problems in the future.

\subsection{Electronic structures}

To understand the electronic structures of conducting polymers, we start the section by introducing atomic orbitals and how they can be developed into band structures.

\subsubsection{Atomic orbital and its hybridization}

Atomic orbitals are the wavefunctions of electrons in an atom obtained by solving the Schrödinger equation for the nucleus-electron multi-body system. Atomic orbitals 
present different states of the electron in an intuitive way, where the geometry of the orbitals schematically show the possibility of finding the electron at a specific position. Atomic orbitals can be described by three quantum numbers: ${ }^{75}$

- Principal quantum number $(n)$ : it describes the energy level or the electron shell (the distance from the nucleus) of an electron. $n$ is an integer number starting from 1 (or capital letter starting from $K$ ).

- Azimuthal or angular quantum number $(l)$ : it represents the subshell of the electron. $l$ defines the overall shape of the electron distribution function (subshell) and takes integer value from 0 to $n-1$ (or lowercase letter $s, p, d$, and $f$ ). The eigen-energy of an atomic orbital is mostly determined by $n$ and $l$.

- Magnetic quantum number $(m)$ : it denotes the specific orientation of an orbital. $m$ is an integer number from $-l$ to $l$ (or subscript described by certain direction in a coordination system like $x, y, z$, etc.).

With all the three quantum numbers, we are able to visualize the shape of atomic orbitals of an atom (Figure 4.7). For $l=0$ (or $s$ ), there is no preferred orientation and the overall shape of the atomic orbitals is a sphere. For $l=1$ (or $p$ ), three configurations are available, each with the overall shape of a "dumbbell" along different axes ${ }^{75}$.

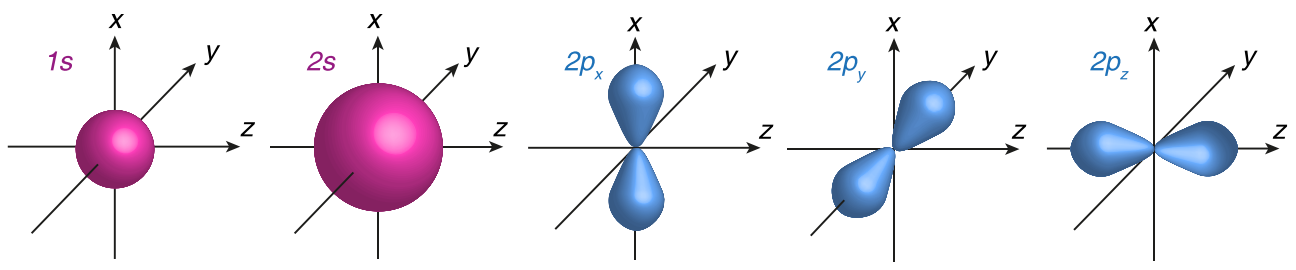

Figure 4.7 | Wavefunctions (or orbitals) of an atom in a three-dimension space. The atomic orbitals for $1 \mathrm{~s}, 2 \mathrm{~s}, 2 \mathrm{p}_{\mathrm{x}}, 2 \mathrm{p}_{\mathrm{y}}$, and $2 \mathrm{p}_{z}$ are exhibited in a Cartesian coordinate system. The figure is modified based on literature ${ }^{75,76}$.

Each atomic orbital is able to accommodate two electrons with opposite spins (Pauli's exclusion principle), and the spin of the electron can be stated by a fourth quantum number, spin quantum number $(s)$. The $\mathrm{H}$ atom has only one electron and in the ground state this electron will occupy $1 s$ atomic orbital. For atoms with larger atomic numbers and more electrons, they have to follow the rules below: 75,77

- Aufbau principle: electrons will first enter the atomic orbitals with lowest available energy levels until these orbitals are fully occupied.

- Hund's rule: every atomic orbital with identical $n$ and $l$ prefers to be singly occupied before any of these orbitals is fully occupied; all electrons in singly occupied orbital share identical spin.

When two atoms move close to each other, atomic orbitals of their valence electrons start to overlap and the mutual interaction "glues" the atoms, creating a covalent bond. The strength of the bond is determined by various factors, including energy differences 
between initial orbitals, spatial orientations of initial orbitals, and overlapping size ${ }^{75}$. The newly formed orbital is defined as molecular orbital, which for simplicity could be regarded as the linear combination of two atomic orbitals ${ }^{75}$. Valence electrons located in initial atomic orbitals then start to accommodate in the molecular orbital.

Carbon $(C)$ is the sixth element in the periodic table of elements and has two core (or inner shell) electrons and four valence (or outermost shell) electrons, as illustrated in Figure 4.8. Five atomic orbitals can be formed in a $C$ atom, as shown in Figure 4.7. In the ground state, only two of them (1s and $2 \mathrm{~s}$ ) are fully occupied by electrons and the remaining two valence electrons have to singly occupy two of the three $2 p$ orbitals. Generally, the chemistry of an atom is determined by its valence electrons ${ }^{75}$. The four valence electrons in a $\mathrm{C}$ atom thus are available to form hybrid orbitals, which was confirmed by experimental evidence ${ }^{75}$.
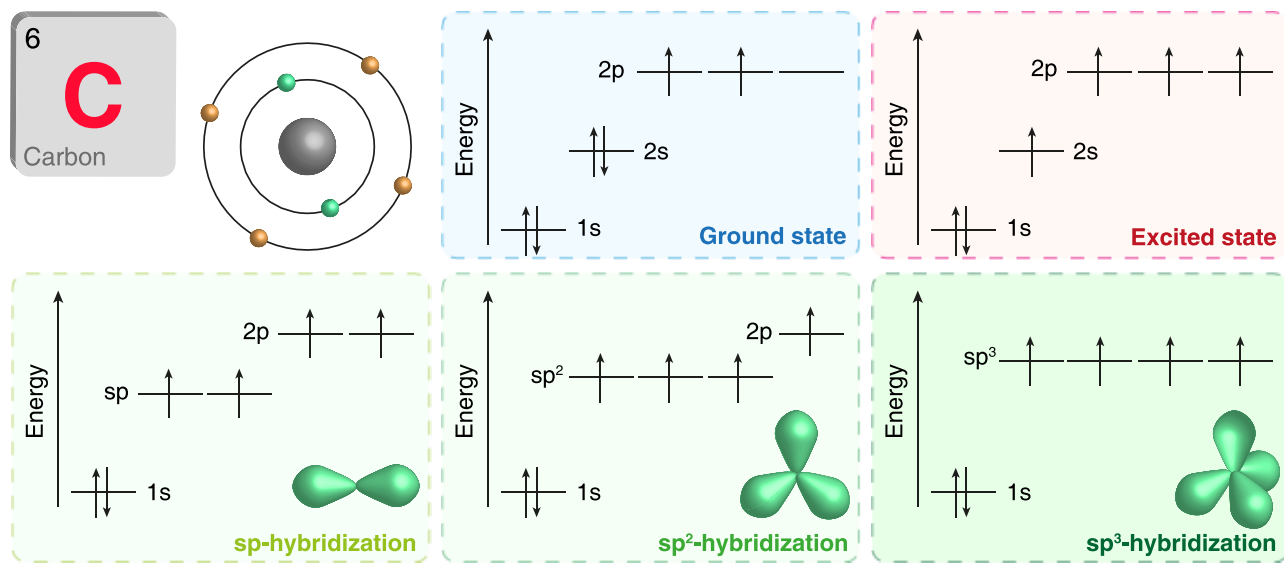

Figure 4.8 | Carbon atom and its atomic orbitals. The orbitals are plotted in the energy scale for ground state, excited state, $\mathrm{sp}$-hybridization, $\mathrm{sp}^{2}$-hybridization, and $\mathrm{sp}^{3}$-hybridization. Insets are schematics of hybridized orbitals in three-dimension space. The figure is modified based on literature $\mathrm{e}^{75,76}$.

The hybrid orbitals are formed between/among atomic orbitals with similar energies inside an atom. The hybridization process is mostly triggered by external electrostatic fields of other atoms (e.g., $\mathrm{H}$ ), which promotes one electron on $2 \mathrm{~s}$ orbital to the third unoccupied $2 p$ orbital (Figure 4.8) creating four unpaired valence electrons (excited state). Three possible configuration of hybrid orbitals can emerge, namely, $\mathrm{sp}^{-}, \mathrm{sp}^{2-}$, and $\mathrm{sp}^{3}$ - hybridization, as indicated in Figure 4.8. The newly created hybrid orbitals share the same energy, which is lower than $2 p$ but higher than $2 s$ orbitals, and provide optimized spatial orientation (or geometry) convenient for bond formation. The gained energy due to the orbital hybridization can be released by the creation of two additional covalent bonds and this process is more favourable thermodynamically.

The direct head-on overlapping of atomic (hybrid) orbitals creates sigma $(\sigma)$ bond and typical examples are the bonds formed between $1 \mathrm{~s}$ orbital of a $\mathrm{H}$ atom and $2 \mathrm{sp}^{2}$ orbital 
of a $C$ atom and between two $2 \mathrm{sp}^{2}$ orbitals of $C$ atoms (Figure 4.9). $\sigma$ bond has the shape of a cylinder along the bond axis, which can be intuitively described as "headto-head" overlapping, where rotation along the bond axis does not break $\sigma$ bond. The two electrons are strongly localized in the area between the two atoms and would not contribute to the electrical properties. For the cases of $\mathrm{sp}$ - and $\mathrm{sp}^{2}$ - hybridization, the remained unhybridized $2 p$ orbitals position perpendicular to the plane or direction of the hybrid orbitals. Two $2 p$ orbitals can form another type of covalent bond, pi $(\pi)$ bond, via orbital interactions in a "side-by-side" way (Figure 4.9). The strength of $\pi$ bonds is weaker than $\sigma$ bonds with electron distributed above and below the bond axis. The two electrons in a $\pi$ bond are delocalized in the whole area of the molecule (or polymer segment) and thus play a key role for electronic conduction in conducting polymers ${ }^{6}$. The coexistence of $\sigma$ and $\pi$ bonds between two atoms form double bonds.
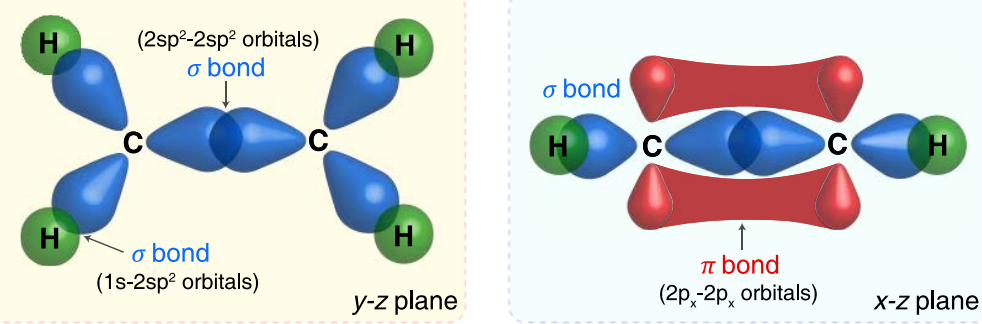

Figure 4.9 | The Formation of $\sigma$ bonds and $\pi$ bonds in an ethylene molecule. $\sigma$ bonds are formed between $\mathrm{C}$ and $\mathrm{C}$ atoms ( $2 \mathrm{sp}^{2}$ and $2 \mathrm{sp}^{2}$ orbitals) or $\mathrm{C}$ and $\mathrm{H}$ atoms (2sp ${ }^{2}$ and 1 s orbitals). $\pi$ bonds are formed between $\mathrm{C}$ and $\mathrm{C}$ atoms ( $2 \mathrm{p}_{\mathrm{x}}$ and $2 \mathrm{p}_{\mathrm{x}}$ orbitals). The figure is modified based on literature ${ }^{75,76}$.
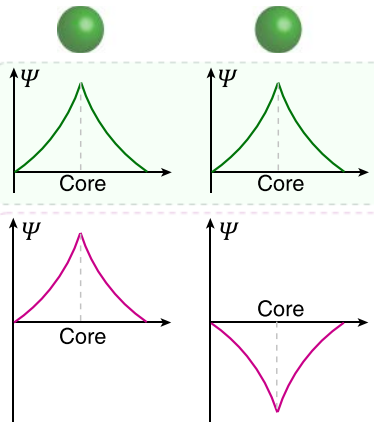
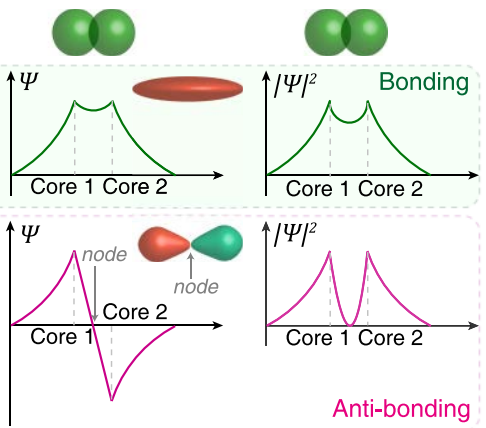

Figure 4.10 | Bonding and anti-bonding orbitals. The sigma bond in the $\mathrm{H}$ atom is formed by two 1 s atomic orbitals, where the corresponding wavefunctions can be combined in constructive or destructive ways. The resulting molecular orbitals are therefore different with zero (bonding) or one (anti-bonding) node. Their positions in energy scale are also plotted. The wavefunctions are approximate curves to illustrate the concept. The figure is modified based on literature $\mathrm{e}^{75,76}$.

The molecular orbitals can be either bonding or anti-bonding, depending on how the two orbitals (wavefunctions) overlap. We can take the sigma bond formation in $\mathrm{H}$ atom 
as an example to illustrate it (Figure 4.10). The wavefunction of the $1 \mathrm{~s}$ orbitals can be either positive-valued or negative-valued and their superposition can therefore be constructive or destructive, giving rise to the bonding $(\sigma)$ or anti-bonding $\left(\sigma^{*}\right)$ molecular orbitals. In bonding orbitals (green colour in Figure 4.10), electrons are mostly located in the area between two atoms, while in anti-bonding orbitals, electrons prefer to escape away from this in-between area (see $/ \Psi / 2$, electron distribution probability function) and a node (zero distribution probability for electrons at this point) is formed.

Comparing with the $\mathrm{H}$ atom, the case of the $\mathrm{C}$ atom can be more complicated with the possibility of bonding $(\pi)$ and anti-bonding $\left(\pi^{*}\right)$ molecular orbital formation by unpaired $2 p$ atomic orbitals. The $\pi$ orbital has a higher energy than the $\sigma$ orbital while the $\pi^{*}$ orbital locates at a lower position than the $\sigma^{*}$ orbital on the energy axis ${ }^{37}$. Based on the Aufbau principle, electrons occupy the low energy orbitals first, and thus, $\pi$ orbital becomes the highest occupied molecular orbital (HOMO) while $\pi^{*}$ orbital becomes the lowest unoccupied molecular orbital (LUMO).

\subsubsection{Formation of energy bands}

In this part, we are moving the discussion to carbon-based molecules and polymers. Figure 4.10 shows that a gap exists between the HOMO and LUMO in the ethylene molecule and an external energy is required to excite electrons from HOMO to LUMO for conduction. This energy gap is related to the electronic coupling strength of the two initial orbitals and can be further reduced by increasing the length of conjugation (alternating single and double bonds), i.e., from butadiene and octatetraene to PA (Figure 4.11). According to theoretical calculations, the gap can be reduced to $1.45 \mathrm{eV}$, when the number of carbon atoms approaches infinity ${ }^{78}$.

For PA with a large number of carbon atoms, the energy spacing between two nearby bonding (or anti-bonding) orbitals is extremely small and negligible since the thermal energy by surroundings at room temperature is sufficiently large to promote electrons to move to the vacancies at higher energy orbitals. Therefore, we consider this bunch of orbitals as a continuous band, where the band formed by $\pi$ orbitals is defined as valence band and the band consisted of $\pi^{*}$ orbitals conduction band (Figure 4.11). The top end of the valence band is the HOMO while the bottom end of conduction band is the LUMO of the polymer. Electrons in the valence band have to gain external energy (e.g., heat, electric field, or light) overcoming the energy gap to be excited to the conduction band for electronic conduction.

The above discussion is based on a single long one-dimensional chain, but for real cases, conducting polymer films are based on three-dimensional stacking with lots of chains. Similar to the discussion for single chains, $\pi-\pi$ intermolecular interactions between $\pi$ orbitals can also create band structures and the band gap can be further reduced. The origin of the band gap in conjugated polymers can be associated with the Peierls instability, which was demonstrated by Rudolf Peierls in 1930s. It was found that a one-dimensional equally spaced chain with one electron per ion cannot be stable ${ }^{29}$. This implies a lattice distortion has to exist for conjugated polymers in 
order to be thermodynamically stable, Therefore, the backbone in conjugated polymers shows bond alternation (alternating bond lengths in the backbone).

Compared to single carbon-carbon bonds in polyethylene (1.52 $\AA$ ), the bond length for single and double bonds in polyacetylene reduces to $1.47 \AA$ and $1.35 \AA$, respectively 79 . It is the bond alternation that opens a gap in the electronic structure. Theoretically, it would be possible to heat the polymer at a certain high temperature to make the length of all bonds equal and form a metallic electronic structure, but such high temperature would decompose the polymer ${ }^{28}$. The Peierls instability makes conjugated polymers show semiconducting instead of metallic properties.

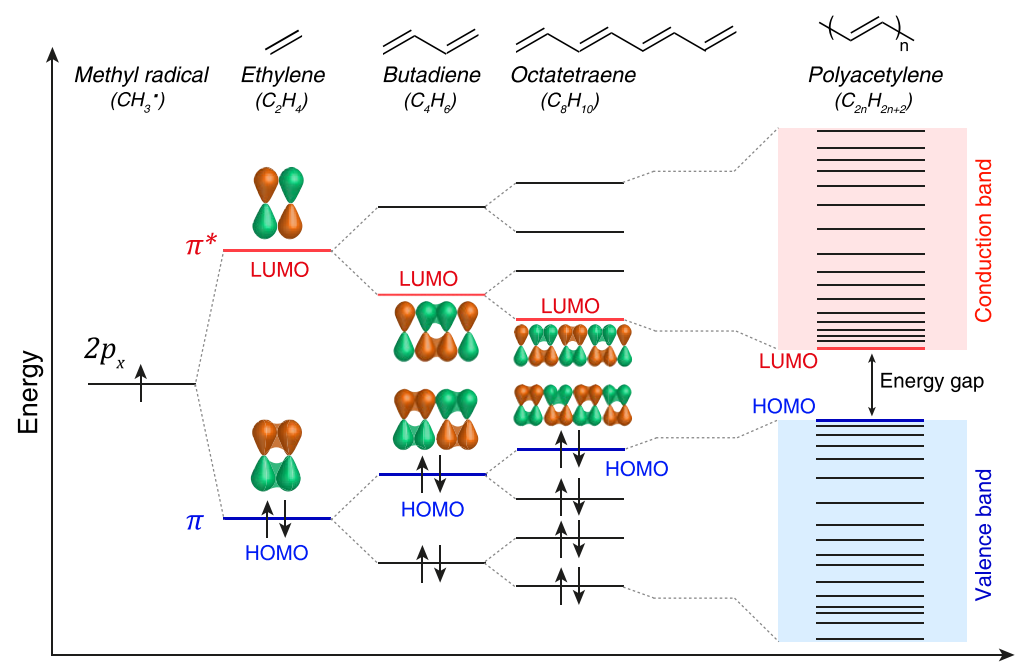

Number of carbon atoms

Figure 4.11 | Energy band formation of PA. 2p $\mathrm{p}_{x}$ atomic orbital of methyl radical $\left(\mathrm{CH}_{3}{ }^{*}\right)$ is split into bonding and anti-bonding orbitals, finally evolving into valence and conduction bands. The energy levels in the figure are just for schematics. The figure is modified based on literature ${ }^{80}$.

The only effective strategy to make conjugated polymers electrically conductive is by doping, introduced in Section 4.1.3. Removing or adding a charge from the polymer moves the HOMO level upwards and a half-occupied polaronic level forms (Figure 4.12). With more and more polarons created in the polymer, polaronic bands can form by interactions between the newly formed polaronic levels ${ }^{28}$. Similar to polaronic levels, polaronic bands are half-occupied in their ground states, showing conductor feature (Figure 2.7). In this case, there is no gap between occupied and unoccupied levels, and the empty spaces in polaronic bands are available for transport of charge carriers.

If two charges are removed in the same segment, a bipolaronic level can form ${ }^{28}$, and with more bipolarons created in the system, these bipolaronic levels will develop into completely empty bipolaronic bands (Figure 4.12) ${ }^{28}$. From the distribution of density of states, the lower bipolaronic band is touching or overlapping with the valence band, making the polymer behave as a semi-metal ${ }^{31}$. A typical example of metallic polymers 
is PANI doped with camphor sulfonic acid (CSA) ${ }^{81}$, and an example of semi-metallic polymer is PEDOT doped with $p$-tolunenesulfonate ${ }^{31}$. The electronic structure of polymers can be characterized by inverse photoemission spectroscopy and ultraviolet photoelectron spectroscopy.

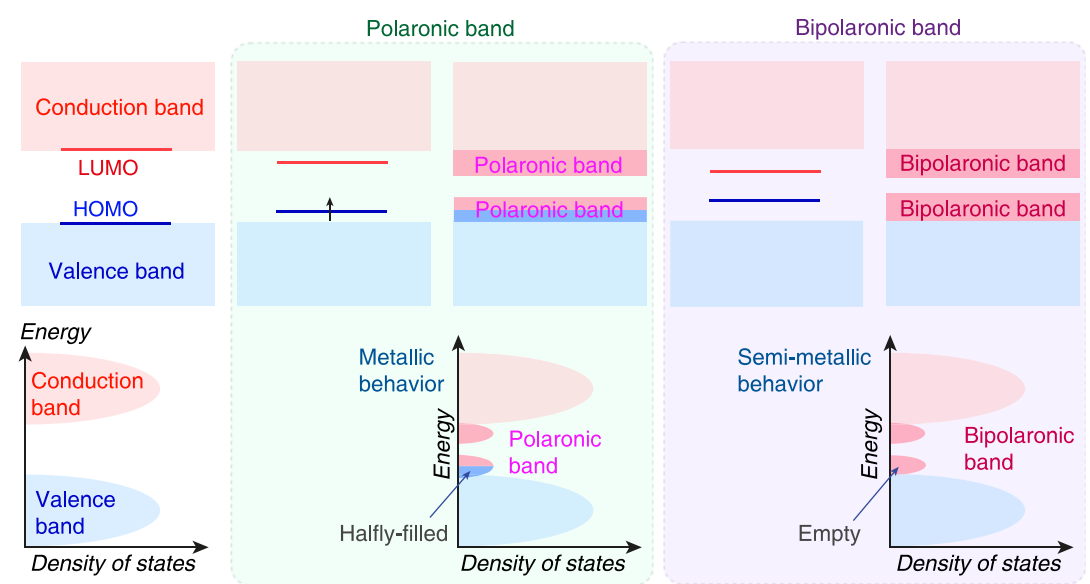

Figure 4.12 | The formation of polaronic and bipolaronic bands by doping. In the figure, the red parts indicate the conduction bands and blue parts valence bands. When one charge is removed from the system, a polaron level is generated. Lots of polarons in the system can create polaronic bands. Similarly, a bipolaron level is formed by the removal of two charges in the system and bipolaronic bands can also be generated by lots of bipolaronic levels. Lower polaronic band is half-occupied while bipolaronic bands are completely empty. The figure is a schematic instead of calculation results and modified based on literature ${ }^{31}$.

\subsection{Charge transport properties of PEDOT}

PEDOT-based materials are currently the most popular conducting polymers utilized in organic (opto-)electronics. PEDOT was first synthesized in the late 1980s by researchers at Bayer for developing antistatic coating materials ${ }^{37,82,83}$. A few years later, PEDOT was dispersed in water with the addition of the counterion PSS, labelled as "Baytron ${ }^{\circledR}$ P". In 2008, it was renamed as "CLEVIOSTM" becoming the product of H. C. Starck, and currently of Heraeus ${ }^{15,37,41}$.

PEDOT is the most popular conducting polymer and one of only a few commercial

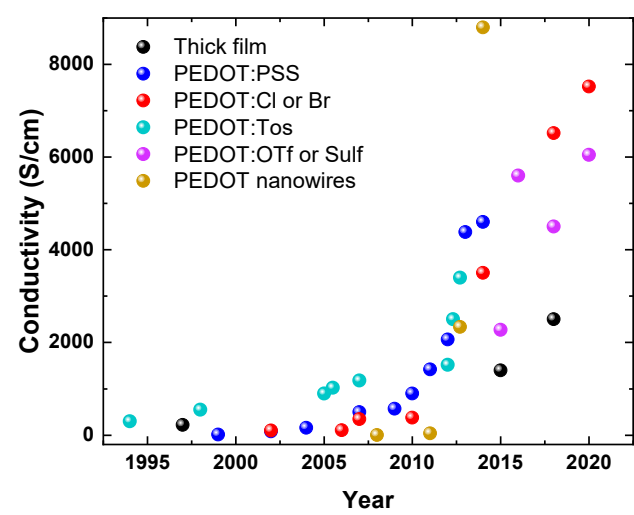

Figure 4.13 | Electrically conductivity of PEDOT-based materials conducting polymers (Section 4.1.5). The outstanding electrical properties of PEDOT 
materials, with great efforts from the whole community (Figure 4.13), make them one of the most suitable model systems for the charge transport study of conducting polymers in this thesis.

\subsubsection{Charge carrier density}

As we stated in Section 2.3.1, the electrical conductivity of a material is governed by its charge carrier density and mobility. The electronic structure of the conducting polymer affects its charge carrier density.

The electronic structure defines the amount of energy levels (rooms) to accommodate the charge carriers. The density of states is defined as the number of states that are available for carrier occupation at a certain energy per unit volume per unit energy 33 . In conducting polymers, the density of states function along the energy axis possesses a parabolic lineshape ${ }^{33}$, as shown in the bottom panel of Figure 4.12.

To calculate the charge carrier density of the material, we also need to know the probablity of energy state occupation by electrons (or holes) of the system. The probability of electron occupation at energy $E$ is defined by the Fermi-Dirac distribution: ${ }^{33}$

$$
f(E)=\frac{1}{e^{\left(E-E_{F}\right) / k_{B} T}+1},
$$

where $E_{F}$ is the Fermi level, where the probability of electron occupation equals to $1 / 2$. In conducting polymers with polaronic bands, $E_{F}$ is at the top of the filled levels in the polaronic band (center); while it is located in the overlapping (or touching) area between the bipolaronic band and the valence band in bipolaronic materials ${ }^{31}$. We use $p$ type conducting polymer as an example to calculate the charge carrier density $\mathrm{p}$ by ${ }^{33}$

$$
p=\int_{E_{\min }}^{E_{\max }} D(E)(1-f(E)) d E,
$$

where $E_{\min }$ and $E_{\max }$ represent the polaronic band minimum and maximum and $D(E)$ is the density of states. The probability of the hole occupation at energy $E$ is $1-f(E)$ for $p$ type polymer. It should be noted that in the real case, the polymer is not in a periodic lattice structure and any distortion of molecular chains can introduce defects levels in the band (or destroy the band structure) and these defect levels can create localized carriers that do not participate in charge transport.

For conducting polymers, the charge carrier density could be measured via Hall effects (for high concentration) and optical spectroscopic methods (Section 5.3) ${ }^{25,43-45}$. Other options include electrochemical methods (e.g., chronocoulometry) which exploit the mixed electronic-ionic properties of the polymers (see Section 5.6.5).

\subsubsection{Crystalline structure and mobility of PEDOT}

The mobility of charge carriers in conducting polymers is heavily affected by the microstructure, e.g., crystallinity and orientation of crystallites. The crystalline structure of PEDOT belongs to the orthorhombic crystal system as illustrated in Figure 4.14. 


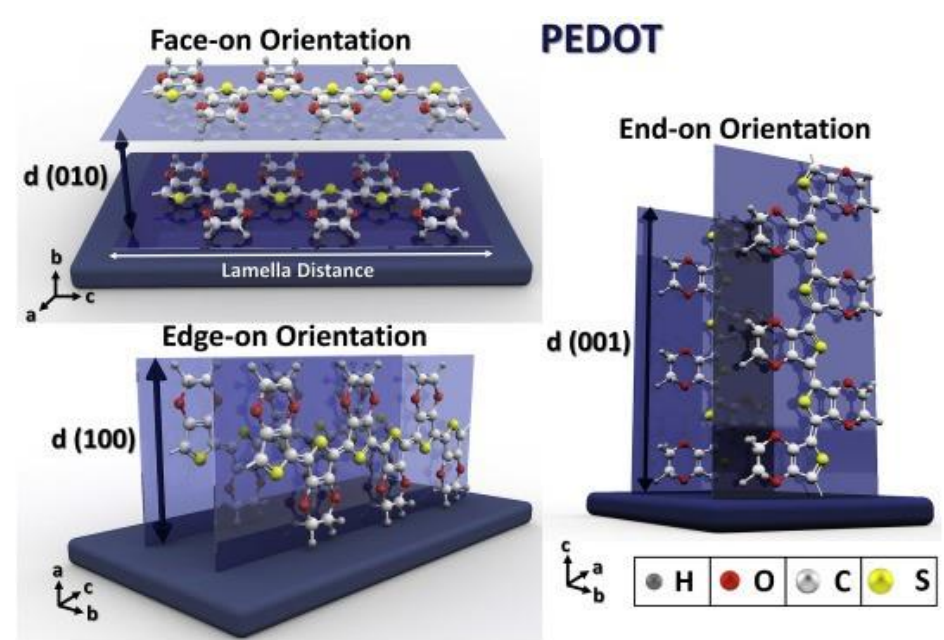

Figure 4.14 | Crystalline structure of PEDOT-based materials. Three possible orientations are presented. The figure is reprinted from Reference 80 under the terms of the Creative Commons CC-BY license.

In the crystal structure of PEDOT, the backbone of a single PEDOT chain is defined along the [001] direction. Two PEDOT chains can stack together via $\pi-\pi$ interaction in [010] direction or be lined up in [100] direction (Figure 4.14). Charge carriers could efficiently travel along [001] via intra-chain transport, while the inter-band transport occurs along [010] or [100] direction. The transport in [100] direction is mostly blocked by counterions ${ }^{84}$. PEDOT films are mostly made of different areas including PEDOT grains and amorphous areas ${ }^{84}$. We could determine the orientation of PEDOT crystals with respect to the substrate via grazing incident wide-angle $x$-ray scatterings (Section 5.5.2). Three orientations, i.e., face-on, edge-on, and end-on, exist for PEDOT crystal grains (Figure 4.14). The VPP PEDOT films in our experiments were mostly in edgeon configuration ${ }^{85}$, which could lead to strong structural and optical anisotropy ${ }^{45}$.

The out-of-plane direction is defined as the direction normal to the substrate and is the [100] direction for edge-on orientation. Charge transport in this direction between polymer chains is blocked by the counterions ${ }^{84}$ and therefore leads to a rather low charge mobility (or electrical conductivity). The orientation of PEDOT crystallites in a film could be influenced by various factors, including deposition methods ${ }^{19}$ and substrate types ${ }^{86}$.

The charge carriers in conducting polymers could have three possible approaches for transport, via band, hopping, or trapassisted transport (Figure 4.15).

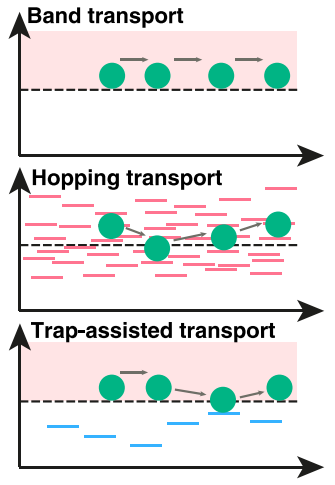

Figure 4.15 I Charge transport models. 
For polymers with ordered structures, delocalized charge carriers can directly move inside the bands without any additional barrier (Figure 4.12). But for conducting polymers with an amorphous microstructure, a large amount of localized charge carriers could only adopt hopping approach for transport between polymer chains. In the situation between the two cases above, traps (or defects) inside the energy gap could assist the charge transport by multiple trapping and releasing processes ${ }^{87}$.

\subsubsection{Boltzmann transport equation}

In this section, we discuss the case of charge transport in a conductor (e.g., metals). The transport theory was proposed by Ludwig Boltzmann, based on a thermodynamic conductor ${ }^{88}$. Although in the discussion the charge carrier used is electron, the theory could also be adapted for most conducting polymers with positively charged polaron as charge carrier. In this theory, electron distribution function $f(\mathbf{r}, \mathbf{k}, t)$ is defined as the probability to find an electron with wavevector (or momentum) of $\mathbf{k}$, at position of $\mathbf{r}$ and time $t$. For metals, the transport equation in the steady state is written as ${ }^{88}$

$$
\left(\frac{\partial f}{\partial t}\right)_{\text {diffusion }}+\left(\frac{\partial f}{\partial t}\right)_{\text {drift }}+\left(\frac{\partial f}{\partial t}\right)_{\text {collisions }}=0 .
$$

Three effects are accounted for in the equation: the diffusion effect due to the carrier density gradient, drift effect due to the external fields, and collisions effects during the transport process due to the carrier scattering events by impurities or phonons. For systems with homogeneous electron density (without any carrier density gradient), the influence from diffusion can be neglected. If we only consider the electric field $\mathbf{E}$, the force can be described by 88

$$
\mathbf{F}=-e \mathbf{E}=\frac{\partial \mathbf{p}}{\partial t}=\frac{\partial \hbar \mathbf{k}}{\partial t},
$$

where $\mathbf{p}$ and $\mathbf{k}$ are the momentum and wavevector of the electron, and $\hbar$ is the reduced Planck constant. Thus, the second term in Equation (4.3) can be described by

$$
\left(\frac{\partial f}{\partial t}\right)_{d r i f t}=\frac{\partial f}{\partial \mathbf{k}} \cdot \frac{\partial \mathbf{k}}{\partial t}=-\frac{e \mathbf{E}}{\hbar} \cdot \frac{\partial f}{\partial \mathbf{k}} .
$$

For the collisions effect, we use the relaxation time approximation that the variation of the distribution function has the simplified form of 88

$$
\left(\frac{\partial f}{\partial t}\right)_{\text {collisions }}=-\frac{f-f^{0}}{\tau_{k}} .
$$

where $\tau_{k}$ is the momentum relaxation time, which is the necessary time for the system to return to its equilibrium state $f^{0}$ through scattering events. Bringing Equation (4.5) and (4.6) back to Equation (4.3) gives the solution of distribution function:

$$
f(k) \approx f^{0}-\frac{e \mathbf{E} \tau_{k}}{\hbar} \cdot \frac{\partial f^{0}}{\partial \mathbf{k}} .
$$

The electrical current density $j$ can be understood as the result of the distribution function shift at the Fermi surface and therefore described by 88

$$
j=\frac{2}{(2 \pi)^{3}} \int d^{3} \mathbf{k}(-e \mathbf{v}) \frac{e \mathbf{E} \tau_{k}}{\hbar} \cdot \frac{\partial f^{0}}{\partial \mathbf{k}} .
$$

Combining Equation (2.28) and (4.8), we have the Boltzmann conductivity ${ }^{88}$ 


$$
\sigma_{B}=\frac{2}{(2 \pi)^{3}} \frac{e^{2}}{\hbar} \int d^{3} \mathbf{k} \tau_{k} v \frac{\partial f^{0}}{\partial \mathbf{k}} .
$$

For spherical Fermi surface (e.g., free electron gas system), $\tau_{k}$ can be approximated as $\mathbf{k}$-independent and therefore, Equation (4.9) can be rewritten as ${ }^{88}$

$$
\sigma_{B}=\frac{e^{2} \tau_{k}}{m} \frac{2}{(2 \pi)^{3}} \int d^{3} \mathbf{k} \boldsymbol{k} \frac{\partial f^{0}}{\partial \mathbf{k}}=\frac{n e^{2} \tau_{k}}{m} .
$$

which is exactly the Drude model formula for DC current which we derived in Section 2.3 using a different approach.

\subsubsection{The Localization-modified Drude model}

We can further investigate the carrier scattering process during charge transport in the material system by considering a charge carrier with wavevector $\mathbf{k}_{\mathbf{F}}$ (point A in Figure 4.16). An external electric field is applied to the system and drives the carrier to move to-wards state $B$ (drift current). This field leads to a change of wavevector direction to its opposite direction $\left(-\mathrm{k}_{\mathrm{F}}\right)$. This process can be realized by either a direct transition or a multiple scattering pathway. As shown in Figure 4.16, there always exist two symmetrical paths (1 or 2) for the multiple scattering processes.

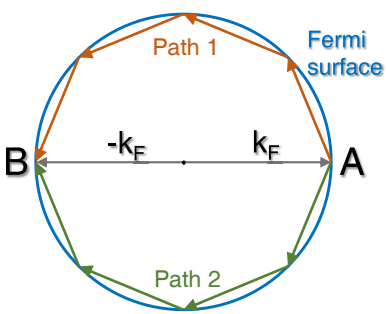

Figure 4.16 I Fermi surface in $\boldsymbol{k}$-space showing multiple scattering paths.

If all scattering processes are elastic, the two paths can have quantum interferences, which are usually neglected in the standard Boltzmann transport above. In 1983, Gerd Bergmann proved that these interferences can modify the electrical conductivity by 89,90

$$
\bmod =\frac{\Delta \sigma}{\sigma}=-\frac{C}{\left(k_{F} l\right)^{2}},
$$

where $C$ is a constant close to $1, l$ is the elastic diffusion length (or mean free path). However, if some collisions are inelastic, the modification becomes ${ }^{90}$

$$
\bmod =\frac{\Delta \sigma}{\sigma}=-\frac{C}{\left(k_{F} l\right)^{2}}\left(1-\frac{l}{L_{i}}\right) .
$$

where $L_{i}$ is the inelastic diffusion length and can be calculated by $(D / \omega)^{1 / 2}$, square root of the product of diffusion constant $D$ (that can be calculated by $D=l^{2} / 3 \tau$ ) and inverse frequency $1 / \omega .{ }^{91,92}$ This effect is normally described as weak localization, which has been observed a lot in amorphous metals ${ }^{90}$. Weak localization becomes dominant when $l$ is small and thus suitable for non-crystalline conductors, such as conducting polymers.

The corrected frequency-dependent conductivity formula based on weak localization is defined as the Localization-modified Drude model (LMD) ${ }^{91,92}$. The real part is ${ }^{91,92}$

$$
\sigma_{L M D}^{\prime}(\omega)=\sigma_{\text {Drude }}^{\prime}(\omega) \cdot\left(1-\frac{C}{\left(k_{F} l\right)^{2}}(1-\sqrt{3 \omega \tau})\right) .
$$

Using Kramers-Kronig transformation, its imaginary part can be derived:91,92 


$$
\sigma_{L M D}^{\prime \prime}(\omega)=\sigma_{\text {Drude }}^{\prime \prime}(\omega) \cdot\left(1+\frac{C}{\left(k_{F} l\right)^{2}}\left(\sqrt{6}-1-\sqrt{\frac{3}{\omega \tau}}\right)\right) .
$$

Their corresponding permittivity (dielectric function) expressions are ${ }^{91,92}$

$$
\begin{gathered}
\varepsilon_{L M D}^{\prime}(\omega)=\varepsilon_{\infty}-\left(1-\varepsilon_{\text {Drude }}^{\prime}(\omega)\right) \cdot\left(1+\frac{C}{\left(k_{F} l\right)^{2}}\left(\sqrt{6}-1-\sqrt{\frac{3}{\omega \tau}}\right)\right), \\
\varepsilon_{\text {LMD }}^{\prime \prime}(\omega)=\varepsilon_{\text {Drude }}^{\prime \prime}(\omega) \cdot\left(1-\frac{C}{\left(k_{F} l\right)^{2}}(1-\sqrt{3 \omega \tau})\right),
\end{gathered}
$$

where the Drude model expression can be found in Equation (2.43) to (2.46) and $\varepsilon_{\infty}$ is the high-energy permittivity to account for effects beyond the measurement range (instead of 1 for the ideal case). Examples of dispersions of permittivity and optical conductivity based on the LMD model are presented in Figure 4.17. By tuning the localization modification parameter, $C /\left(\mathrm{kF}_{\mathrm{F}}\right)^{2}$, we can introduce an increasing trend of the real permittivity at low frequencies while the optical conductivity as well as DC conductivity decreases. This modification narrows the negative real permittivity range and increases the imaginary permittivity amplitude. Hence, this localization effect in a material is detrimental in terms of plasmonic properties (see Section 3.3).
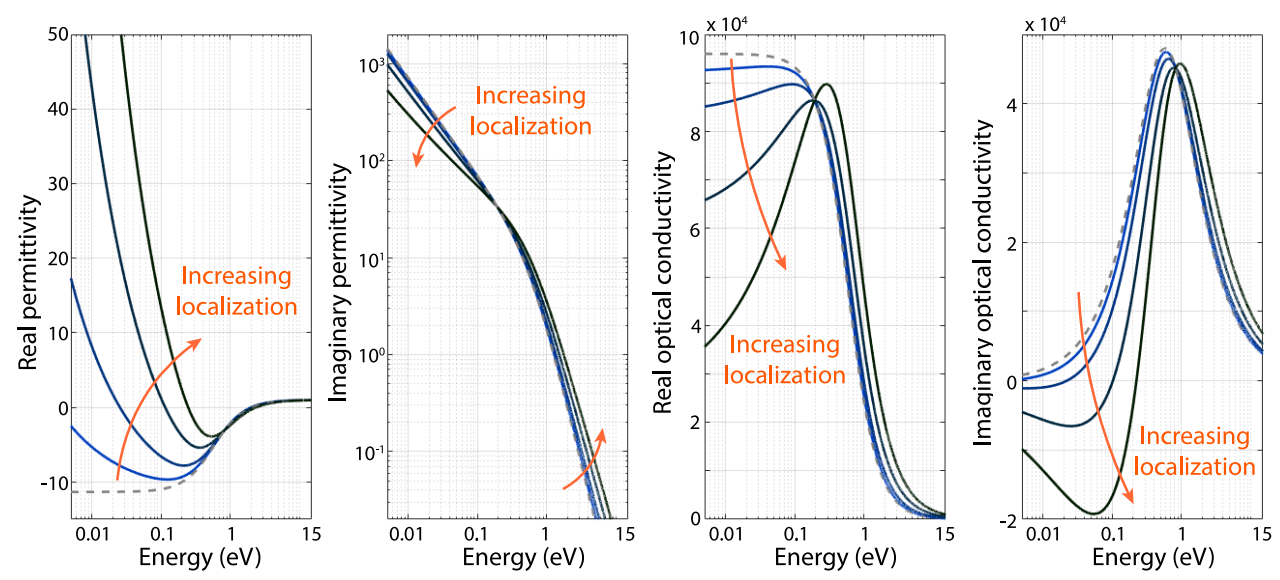

Figure 4.17 | Localization-modified Drude model. The dashed lines present Drude model without any modification.

The LMD model was first introduced to conducting polymer in the study of polyaniline doped with $\mathrm{CSA}^{91}$, to describe its anomalous optical conductivity behaviour deviating from the Drude model. It was later applied for other conducting polymers, including polypyrrole ${ }^{92-94}$, and PEDOT $44,95-97$. However, within the LMD model, the DC real permittivity becomes unphysical, since the permi-ttivity function diverges for $\omega=0$ (i.e., $\varepsilon_{L M D}^{\prime}(\omega) \rightarrow \infty$ for $\left.\omega \rightarrow 0\right)$. 


\subsubsection{The Drude-Smith model}

The optical conductivity and permittivity of poor metals, such as liquid metals (mercury) and metal alloys, were studied by N. V. Smith in late $1960 \mathrm{~s}^{98}$. A generalized formula to describe their deviation from the Drude model was proposed by him in 2001, which was later named as the Drude-Smith model ${ }^{99}$.

The model was derived based on the impulse response function and Poisson statistics, and the modification term of the Drude model is introduced to describe the effect of carrier collision during charge transport, which leads to a change of their movement direction. The model can be expressed by ${ }^{99}$

$$
\begin{gathered}
\sigma_{D S}(\omega)=\sigma_{\text {Drude }}(\omega) \cdot\left(1+\sum_{n=1}^{\infty} \frac{C_{n}}{(1-i \omega \tau)^{n}}\right), \\
\varepsilon_{D S}(\omega)=\varepsilon_{\infty}-\left(1-\varepsilon_{\text {Drude }}(\omega)\right) \cdot\left(1+\sum_{n=1}^{\infty} \frac{C_{n}}{(1-i \omega \tau)^{n}}\right),
\end{gathered}
$$

where $C_{n}$ is a modification coefficient indicating the fractions of retained initial velocity after the $n$th collision. To make the model fit to experimental data, $C_{n}$ always has to be a negative value between 0 and -1 . This implies collision is expected to not only reduce the velocity but also backscatter the carrier in its opposite direction. In most studies, only the $1^{\text {st }}$ collision was considered ${ }^{99,100}$.
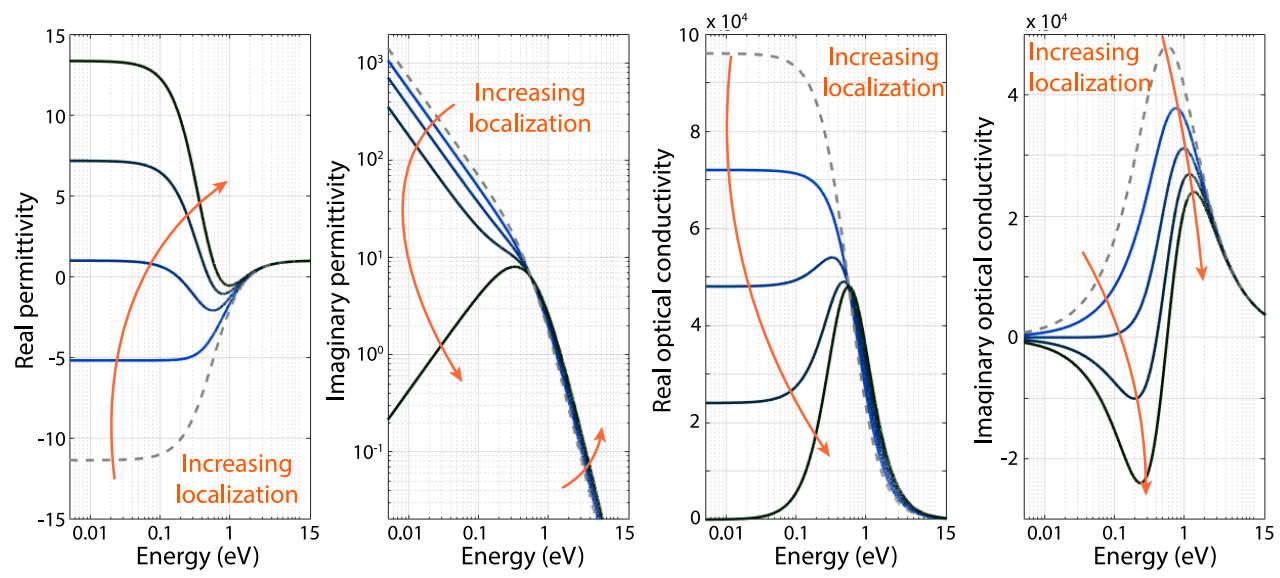

Figure 4.18 | Drude-Smith model. The dashed lines present Drude model with no modification. The orange arrows indicate the change of permittivity and optical conductivity with the increase of localization parameter $\mathrm{C}_{1}$.

Examples of dispersion curves of the Drude-Smith model are shown in Figure 4.18. The effect of increasing the (negative) modification coefficient is similar to the behavior of the LMD model. However, for this model the permittivity modification has an upper limit. As shown in Figure 4.18, the maximal modification of the real permittivity is achieved when $C_{1}$ is -1 (darkest curve) at low frequencies. 
An issue of the Drude-Smith model is the unclear physical origin for the backscattering of charge carriers. The carrier backscattering indeed reduces the DC conductivity and produces the "anomalous" optical conductivity dispersion at low frequencies for liquid metals ${ }^{99}$. However, there is still a lack of reasonable explanations for such modification. The Drude-Smith model was frequently utilized for conducting polymers, especially for interpretating results from THz-TDS spectroscopy measurements ${ }^{101-103}$.

\subsubsection{The Drude-Lorentz model}

Although both the LMD and the Drude-Smith models were proposed for conducting polymers, they have several limitations, such as the unclear physical meaning for their modifications. In addition, these models were originally developed for metals (glassy or liquid metals) for infrared ranges. Therefore, interband transitions and molecular vibrations in polymers were not taken into consideration. In turn, both interband transitions and molecular vibrations can induce large variations of the permittivity and affect the overall lineshape of the dispersion curves in the infrared and even lower frequency ranges ${ }^{45,104-106 .}$

In order to address these issues, we developed the Drude-Lorentz model and used it for conducting polymers. The Drude-Lorentz model is expressed by: 45

$$
\begin{aligned}
& \varepsilon_{D L}(\omega)=\varepsilon_{D}(\omega)-\sum \frac{A_{L}}{\omega^{2}-\omega_{L}^{2}+i \omega \gamma_{L}}, \\
& \sigma_{D L}(\omega)=\sigma_{D}(\omega)+\sum \frac{i \varepsilon_{0} \omega A_{L}}{\omega^{2}-\omega_{L}^{2}+i \omega \gamma_{L}},
\end{aligned}
$$

both of which are a linear combination of the Drude formula and Lorentz oscillators (see Section 2.3). The different Lorentz oscillators, located at different spectral ranges and with different broadening and amplitudes, can provide useful information on the physical processes in conducting polymers, such as interband transitions, molecular vibrations, localized carriers, and unknown processes in the $\mathrm{THz}$ range ${ }^{45}$. From the Drude component, we could obtain the charge carrier density, mobility, and electrical conductivity of the films ${ }^{45}$. More details can be found in Section 6.1 and Paper 1.

\subsection{References}

1 Jensen, W. B. The origin of the polymer concept. Journal of Chemical Education 85, 624 (2008).

2 Chapman, D. Inorganic Polymers. Nature 198, 50-50 (1963).

3 Guo, X. \& Facchetti, A. The journey of conducting polymers from discovery to application. Nature Materials 19, 922-928 (2020).

4 Shirakawa, H., Louis, E. J., MacDiarmid, A. G., Chiang, C. K. \& Heeger, A. J. Synthesis of electrically conducting organic polymers: halogen derivatives of polyacetylene, $(\mathrm{CH}) \times$. Journal of the Chemical Society, Chemical Communications, 578-580 (1977).

5 Chiang, C. K. et al. Electrical conductivity in doped polyacetylene. Physical Review Letters 39, 1098 (1977). 
Heeger, A. J. Semiconducting and metallic polymers: the fourth generation of polymeric materials (Nobel lecture). Angewandte Chemie International Edition 40, 25912611 (2001).

MacDiarmid, A. G. "Synthetic metals": a novel role for organic polymers (Nobel lecture). Angewandte Chemie International Edition 40, 2581-2590 (2001).

Shirakawa, $\mathrm{H}$. The discovery of polyacetylene film: the dawning of an era of conducting polymers (Nobel lecture). Angewandte Chemie International Edition 40, 2574-2580 (2001).

Rasmussen, S. C. in 100+ Years of Plastics. Leo Baekeland and Beyond (ACS Publications, 2011).

Nobel-Media. The Nobel Prize in Chemistry 2000, <https://www.nobelprize.org/prizes/ chemistry/2000/prize-announcement/> (2000).

Rasmussen, S. C. New Insight into the "Fortuitous Error" that Led to the 2000 Nobel Prize in Chemistry. Substantia, doi:10.13128/Substantia-973 (2020).

Chiang, C. K. et al. Polyacetylene, $(\mathrm{CH})$ : : n-type and p-type doping and compensation. Applied Physics Letters 33, 18-20 (1978).

Tsukamoto, J., Takahashi, A. \& Kawasaki, K. Structure and electrical properties of polyacetylene yielding a conductivity of $10^{5} \mathrm{~S} / \mathrm{cm}$. Japanese Journal of Applied Physics 29, 125 (1990).

Deits, W., Cukor, P., Rubner, M. \& Jopson, H. Stability and stabilization of polyacetylene, polyphenylacetylene, and acetylene/phenylacetylene copolymers. Synthetic Metals 4, 199-210 (1982).

(CRC Press Taylor \& Francis Group, 2019).

16 Wang, Y. et al. A highly stretchable, transparent, and conductive polymer. Science advances 3, e1602076 (2017).

Worfolk, B. J. et al. Ultrahigh electrical conductivity in solution-sheared polymeric transparent films. Proceedings of the National Academy of Sciences 112, 14138-14143 (2015).

Wang, G. et al. Aggregation control in natural brush-printed conjugated polymer films and implications for enhancing charge transport. Proceedings of the National Academy of Sciences 114, E10066-E10073 (2017).

Wang, $X$. et al. High electrical conductivity and carrier mobility in oCVD PEDOT thin films by engineered crystallization and acid treatment. Science advances 4, eaat5780 (2018).

Ugur, A. et al. Low-dimensional conduction mechanisms in highly conductive and transparent conjugated polymers. Advanced Materials 27, 4604-4610 (2015).

Cho, B. et al. Single-crystal poly (3, 4-ethylenedioxythiop
high conductivity. Nano Letters 14, 3321-3327 (2014). Kim, G.-H., Shao, L., Zhang, K. \& Pipe, K. P. Engineered doping of organic semiconductors for enhanced thermoelectric efficiency. Nature Materials 12, 719-723 (2013). Crispin, X. et al. The origin of the high conductivity of poly (3, 4-ethylenedioxythiophene)-poly(styrenesulfonate)(PEDOT-PSS) plastic electrodes. Chemistry of Materials 18, 4354-4360 (2006).

Bubnova, O. et al. Optimization of the thermoelectric figure of merit in the conducting polymer poly (3, 4-ethylenedioxythiophene). Nature Materials 10, 429-433 (2011).

25 Xia, Y., Sun, K. \& Ouyang, J. Solution-processed metallic conducting polymer films as transparent electrode of optoelectronic devices. Advanced Materials 24, 2436-2440 (2012).

26 Kim, N. et al. Highly conductive PEDOT: PSS nanofibrils induced by solution-processed crystallization. Advanced Materials 26, 2268-2272 (2014).
} 
27 Vijayakumar, V. et al. Bringing conducting polymers to high order: Toward conductivities beyond $10^{5} \mathrm{~S} \mathrm{~cm}^{-1}$ and thermoelectric power factors of $2 \mathrm{mw} \mathrm{m}^{-1} \mathrm{k}^{-2}$. Advanced Energy Materials 9, 1900266 (2019).

Bredas, J. L. \& Street, G. B. Polarons, bipolarons, and solitons in conducting polymers. Accounts of Chemical Research 18, 309-315 (1985). Kittel, C. Introduction to solid state physics. ( John Wiley \& Sons, 1996). $\mathrm{Xu}, \mathrm{K}$. et al. Ground-state electron transfer in all-polymer donor-acceptor heterojunctions. Nature Materials 19, 738-744 (2020).

Bubnova, O. et al. Semi-metallic polymers. Nature Materials 13, 190-194 (2014). Heeger, A. J. Charge storage in conducting polymers: solitons, polarons, and bipolarons. Polymer journal 17, 201-208 (1985).

Sze, S. M. Semiconductor devices: physics and technology. (John wiley \& sons, 2008). Kiefer, D. et al. Double doping of conjugated polymers with monomer molecular dopants. Nature Materials 18, 149-155 (2019). PEDOT: PSS for its application as transparent electrode of optoelectronic devices. Displays 34, 423-436 (2013). hexylthiophene) through understanding the correlation with solid-state order. Macromolecules 50, 8140-8148 (2017).

7 Elschner, A., Kirchmeyer, S., Lovenich, W., Merker, U. \& Reuter, K. PEDOT: principles and applications of an intrinsically conductive polymer. (CRC press, 2010).

8 Palumbiny, C. M. et al. The crystallization of PEDOT: PSS polymeric electrodes probed in situ during printing. Advanced materials 27, 3391-3397 (2015).

9 Persson, K. M. et al. Electronic control of cell detachment using a self-doped conducting polymer. Advanced Materials 23, 4403-4408 (2011).

Karlsson, R. H. et al. Iron-catalyzed polymerization of alkoxysulfonate-functionalized 3, 4-ethylenedioxythiophene gives water-soluble poly (3, 4-ethylenedioxythiophene) of high conductivity. Chemistry of Materials 21, 1815-1821 (2009). Shi, H., Liu, C., Jiang, Q. \& Xu, J. Effective approaches to improve the electrical conductivity of PEDOT: PSS: a review. Advanced Electronic Materials 1, 1500017 (2015).

Zhang, L. et al. The role of mineral acid doping of PEDOT: PSS and its application in organic photovoltaics. Advanced Electronic Materials 6, 1900648 (2020). Wu, F. et al. Conductivity enhancement of PEDOT: PSS via addition of chloroplatinic acid and its mechanism. Advanced Electronic Materials 3, 1700047 (2017). $\mathrm{Kim}, \mathrm{N}$. et al. Role of interchain coupling in the metallic state of conducting polymers. Physical Review Letters 109, 106405 (2012).

5 Chen, S. et al. On the anomalous optical conductivity dispersion of electrically conducting polymers: ultra-wide spectral range ellipsometry combined with a Drude-Lorentz model. Journal of Materials Chemistry C 7, 4350-4362 (2019).

47 Advanced Materials 25, $4488-4493$ (2013). Materials Chemistry C 5, 5824-5830 (2017).

48 Rivnay, J. et al. Organic electrochemical transistors. Nature Reviews Materials 3, 1-14 (2018).

49 Jager, E. W. H., Smela, E. \& Inganäs, O. Microfabricating conjugated polymer actuators. Science 290, 1540-1545 (2000).

50 Bubnova, O., Berggren, M. \& Crispin, X. Tuning the thermoelectric properties of conducting polymers in an electrochemical transistor. Journal of the American Chemical Society 134, 16456-16459 (2012). 
51 Sonmez, G., Sonmez, H. B., Shen, C. K. F. \& Wudl, F. Red, green, and blue colors in polymeric electrochromics. Advanced Materials 16, 1905-1908 (2004).

Zhang, S. \& Cicoira, F. Water-Enabled Healing of Conducting Polymer Films. Advanced Materials 29, 1703098 (2017).

53 Rivnay, J. et al. Structural control of mixed ionic and electronic transport in conducting polymers. Nature Communications 7, 1-9 (2016).

54 Stavrinidou, E. et al. Engineering hydrophilic conducting composites with enhanced ion mobility. Physical Chemistry Chemical Physics 16, 2275-2279 (2014).

Paulsen, B. D. et al. Time-Resolved Structural Kinetics of an Organic Mixed IonicElectronic Conductor. Advanced Materials 32, 2003404 (2020).

Jiang, Y., Liu, T. \& Zhou, Y. Recent Advances of Synthesis, Properties, Film Fabrication Methods, Modifications of Poly (3, 4-ethylenedioxythiophene), and Applications in Solution-Processed Photovoltaics. Advanced Functional Materials 30, 2006213 (2020).

Meredith, P. \& Armin, A. Scaling of next generation solution processed organic and perovskite solar cells. Nature Communications 9, 1-4 (2018).

Bao, Z., Dodabalapur, A. \& Lovinger, A. J. Soluble and processable regioregular poly (3-hexylthiophene) for thin film field-effect transistor applications with high mobility. Applied physics letters 69, 4108-4110 (1996).

Reese, C., Roberts, M., Ling, M.-m. \& Bao, Z. Organic thin film transistors. Materials Today 7, 20-27 (2004).

\section{9-385 (2016).}

Chortos, A., Liu, J. \& Bao, Z. Pursuing prosthetic electronic skin. Nature Materials 15, 937-950 (2016). tronics for neuromorphic computing. Nature Electronics 1, 386-397 (2018). Kim, B., Hwang, J. U. \& Kim, E. Chloride transport in conductive polymer films for an n-type thermoelectric platform. Energy \& Environmental Science 13, 859-867 (2020). Malti, A. et al. An organic mixed ion-electron conductor for power electronics. Advanced Science 3, 1500305 (2016).

Khodagholy, D. et al. Organic electronics for high-resolution electrocorticography of the human brain. Science Advances 2, e1601027 (2016).

6 Jonsson, A. et al. Bioelectronic neural pixel: Chemical stimulation and electrical sensing at the same site. Proceedings of the National Academy of Sciences 113, 94409445 (2016).

Khodagholy, D. et al. NeuroGrid: recording action potentials from the surface of the brain. Nature Neuroscience 18, 310-315 (2015).

68 Poxson, D. J. et al. Regulating plant physiology with organic electronics. Proceedings of the National Academy of Sciences 114, 4597-4602 (2017).

69 Jonsson, A., Sjöström, T. A., Tybrandt, K., Berggren, M. \& Simon, D. T. Chemical delivery array with millisecond neurotransmitter release. Science advances 2, e1601340 (2016).

70 Zabihipour, M. et al. High yield manufacturing of fully screen-printed organic electrochemical transistors. npj Flexible Electronics 4, 159 (2020).

71 Andersson Ersman, P. et al. All-printed large-scale integrated circuits based on organic electrochemical transistors. Nature Communications 10, 5053 (2019).

72 Han, S. et al. Thermoelectric polymer aerogels for pressure-temperature sensing applications. Advanced Functional Materials 27, 1703549 (2017).

73 Han, S. et al. A multiparameter pressure-temperature-humidity sensor based on mixed ionic-electronic cellulose aerogels. Advanced Science 6, 1802128 (2019).

74 Lu, B. et al. Pure PEDOT: PSS hydrogels. Nature Communications 10, 1-10 (2019). 
Atkins, P. \& De Paula, J. Elements of physical chemistry. (Macmillan, 2009).

Brown, T. L. Chemistry: the central science. (Pearson Education, 2009).

Griffiths, D. J. \& Schroeter, D. F. Introduction to quantum mechanics. (Cambridge University Press, 2018).

Bredas, J.-L., Silbey, R., Boudreaux, D. S. \& Chance, R. R. Chain-length dependence of electronic and electrochemical properties of conjugated systems: polyacetylene, polyphenylene, polythiophene, and polypyrrole. Journal of the American Chemical Society 105, 6555-6559 (1983).

Yokojima, S. \& Chen, G. Linear scaling calculation of excited-state properties of polyacetylene. Physical Review B 59, 7259 (1999).

Gharahcheshmeh, M. H. \& Gleason, K. K. Texture and nanostructural engineering of conjugated conducting and semiconducting polymers. Materials Today Advances 8, 100086 (2020).

Lee, K. et al. Metallic transport in polyaniline. Nature 441, 65-68 (2006).

Bayer, A. G. Polythiophenes, process for their preparation and their use. (1988).

Groenendaal, L., Jonas, F., Freitag, D., Pielartzik, H. \& Reynolds, J. R. Poly (3, 4-ethylenedioxythiophene) and its derivatives: past, present, and future. Advanced materials 12, 481-494 (2000).

4 Aasmundtveit, K. E. et al. Structure of thin films of poly (3, 4-ethylenedioxythiophene). Synthetic Metals 101, 561-564 (1999).

Chen, S. et al. Unraveling vertical inhomogeneity in vapour phase polymerized PEDOT: Tos films. Journal of Materials Chemistry A 8, 18726-18734 (2020).

Franco-Gonzalez, J. F., Rolland, N. \& Zozoulenko, I. V. Substrate-dependent morphology and its effect on electrical mobility of doped poly (3, 4-ethylenedioxythiophene) (PEDOT) thin films. ACS applied materials \& interfaces 10, 29115-29126 (2018).

8 Simonetti, O. \& Giraudet, L. Transport models in disordered organic semiconductors and their application to the simulation of thin-film transistors. Polymer International 68, 620-636 (2019).

8 Datta, S. Electronic transport in mesoscopic systems. (Cambridge university press, 1997).

9 Bergmann, G. Physical interpretation of weak localization: A time-of-flight experiment with conduction electrons. Physical Review B 28, 2914 (1983).

Mott, N. Metal-insulator transitions. (CRC Press, 2004).

1 Lee, K., Heeger, A. J. \& Cao, Y. Reflectance of polyaniline protonated with camphor sulfonic acid: disordered metal on the metal-insulator boundary. Physical Review B 48, 14884 (1993).

2 Lee, K., Menon, R., Yoon, C. O. \& Heeger, A. J. Reflectance of conducting polypyrrole: Observation of the metal-insulator transition driven by disorder. Physical Review B $\mathbf{5 2}$, 4779 (1995).

3 Lee, K. et al. Nature of the metallic state in conducting polypyrrole. Advanced Materials 10, 456-459 (1998).

4 Kohlman, R. S. et al. Limits for metallic conductivity in conducting polymers. Physical Review Letters 78, 3915 (1997).

5 Chang, Y., Lee, K., Kiebooms, R., Aleshin, A. \& Heeger, A. J. Reflectance of conducting poly (3, 4-ethylenedioxythiophene). Synthetic metals 105, 203-206 (1999).

6 Cho, S., Park, S. \& Lee, K. Reflectance study on the metal-insulator transition driven by crystallinity change in poly (3, 4-ethylenedioxy thiophene)/poly (styrenesulfonate) films. Journal of the Korean Physical Society 47, 474-478 (2005).

Yamashita, M., Otani, C., Shimizu, M. \& Okuzaki, H. Effect of solvent on carrier transport in poly (3, 4-ethylenedioxythiophene)/poly(4-styrenesulfonate) studied by terahetz and infrared-ultraviolet spectroscopy. Applied physics letters 99, 213 (2011). 
98 Smith, N. V. Drude theory and the optical properties of liquid mercury. Physics Letters A 26, 126-127 (1968).

99 Smith, N. V. Classical generalization of the Drude formula for the optical conductivity. Physical Review B 64, 155106 (2001).

100 Noriega, R. et al. A general relationship between disorder, aggregation and charge transport in conjugated polymers. Nature Materials 12, 1038-1044 (2013).

101 Unuma, T., Fujii, K., Kishida, H. \& Nakamura, A. Terahertz complex conductivities of carriers with partial localization in doped polythiophenes. Applied Physics Letters 97 , 155 (2010).

102 Yan, F., Parrott, E. P. J., Ung, B. S. Y. \& Pickwell-MacPherson, E. Solvent doping of PEDOT/PSS: effect on terahertz optoelectronic properties and utilization in terahertz devices. The Journal of Physical Chemistry C 119, 6813-6818 (2015).

103 Du, Y. et al. Dielectric Properties of DMSO-Doped-PEDOT: PSS at THz Frequencies. physica status solidi (b) 255, 1700547 (2018).

104 Schubert, M. et al. Infrared ellipsometry characterization of conducting thin organic films. Thin Solid Films 455, 295-300 (2004).

105 Schubert, M. et al. Carrier redistribution in organic/inorganic (poly (3, 4-ethylenedioxy thiophene/poly (styrenesulfonate) polymer)-Si) heterojunction determined from infrared ellipsometry. Applied physics letters 84, 1311-1313 (2004).

106 Pettersson, L. A. A., Carlsson, F., Inganäs, O. \& Arwin, H. Spectroscopic ellipsometry studies of the optical properties of doped poly (3, 4-ethylenedioxythiophene): an anisotropic metal. Thin Solid Films 313, 356-361 (1998). 


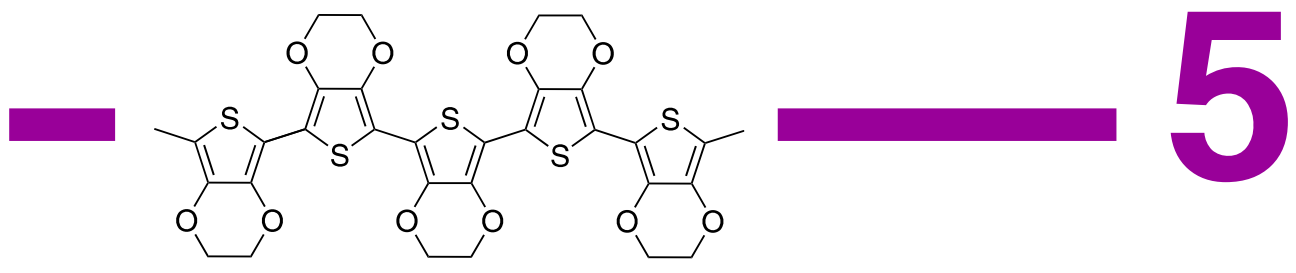

Methods and Characterization

In this chapter, our focus will move on to more practical parts of the study concerning the fabrication and characterization of PEDOT thin films and nanostructures. Methods for deposition and nanostructuring of conducting polymers, and techniques on optical, electrical properties and microstructure characterization will be introduced in detail.

\section{Contents}

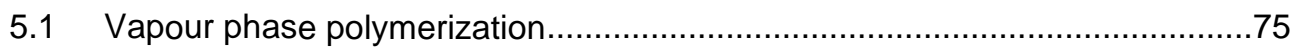

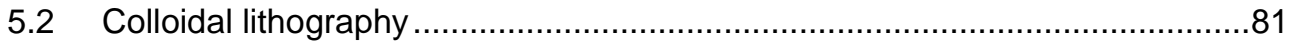

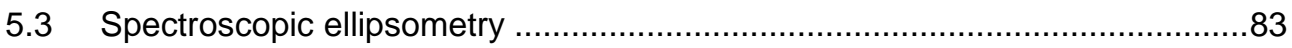

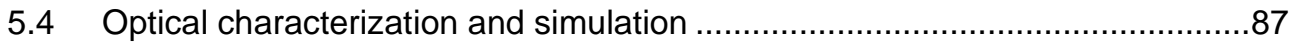

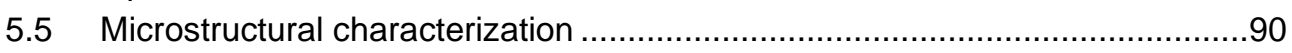

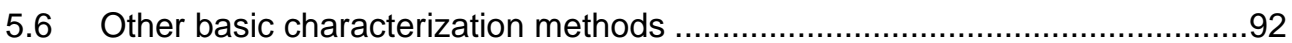

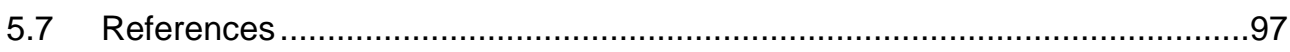

\subsection{Vapour phase polymerization}

Oxidative polymerization is a commonly used way for conducting polymer synthesis. In this method, oxidants are introduced into the system to initialize the polymerization of monomers. For PEDOT synthesis, three steps are included, as shown in Figure 5.1. ${ }^{1,2}$ In brief, 3-4-ethylenedioxythiophene (EDOT) monomers are first transformed 
into radicals by oxidants (step 1). Two obtained radicals can react to form a dimer, and with the assistance of base, the dimer can further be stabilized by deprotonation (step 2). In a similar way, dimers can grow into polymer chains, and the oxidant can further p-dope the polymer by removal of charges in the backbone (step 3). Monomers and oxidants are two basic precursors for the reaction, and the physical state in which they are supplied defines the method of polymerization. For instance, in-situ chemical polymerization (iCP) requires monomers and oxidants that dissolve in solvent ${ }^{3-6}$, while oxidative chemical vapour deposition (CVD) requires both to be in vapour state th $^{7-9}$. Electrochemical polymerization is similar to oxidative polymerization, but some of the steps are assisted by an electrochemical bias, instead of purely oxidants ${ }^{10}$.

\section{Step 1: Formation of radicals Step 2: Dimerization and deprotonation}

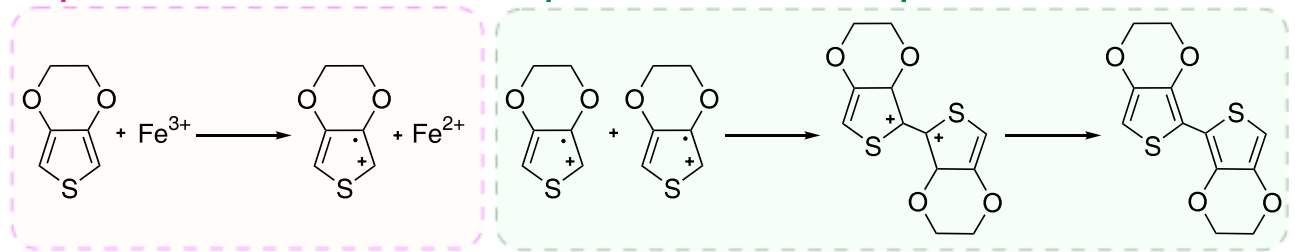

Step 3: Polymerization and oxidation

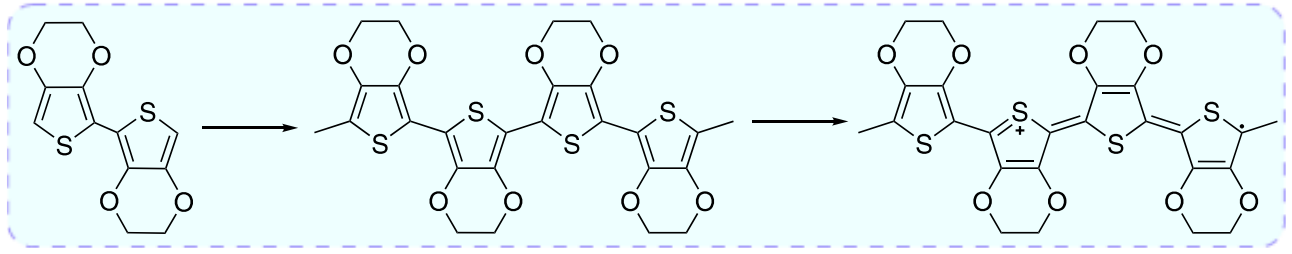

Figure 5.1 | Oxidative polymerization process for PEDOT. Three steps are included to make monomers become dimers, and finally oxidized polymers. The figure is modified from literature ${ }^{1}$.

Vapour phase polymerization (VPP) can be simply regarded as a hybrid mode of iCP and CVD, because in this method the monomers are provided in vapour while the oxidants are in solid state (thin film) ${ }^{11,12}$. Conducting polymers prepared by VPP show excellent properties and have been used for many applications, from optoelectronics ${ }^{13}$ and thermoelectrics ${ }^{14}$ to supercapacitors ${ }^{15}$. Compared to iCP, the morphology ${ }^{2,16,17}$, texture alignment ${ }^{18-21}$, and nanostructures ${ }^{22,23}$ of oxidant films in VPP provides additional dimension for the control of polymer growth. The oxidant in VPP is typically prepared by spin-coating, instead of vaporization in CVD, which offers more options of possible counterions to use, from iron(III) chloride, iron(III) $p$-toluenesulfonate to iron(III) trifluoro-methanesulfonate ${ }^{24}$. Thus, VPP is one of the best choices to prepare high-performance conducting polymers and it was the method used in this study.

\subsubsection{Procedure of VPP}

The process flow of VPP is presented in Figure 5.2. A solution of oxidant and additives are dissolved in certain solvents (normally ethanol and sometimes with co-solvent ${ }^{4}$ ) 
and spin coated on a pre-cleaned substrate (step 1). Thermal annealing during a short time (e.g., 30 seconds) is applied after spin coating to evaporate the ethanol and excessive water contents. After this, the sample is transferred into a vacuum chamber filled with the vapour of monomers (step 2). The vapour is produced by mildly heating monomer droplets on a heating controller inside the chamber. During the polymerization, the pressure and temperature of the chamber and the reaction time could be controlled based on needs (step 3). The standard polymerization time that we used was normally 0.5 to 1 hour depending on the desired film thickness. It should be noted here that the depletion of monomers could terminate the reaction if the volume of monomers is too low or the processing time is too long.
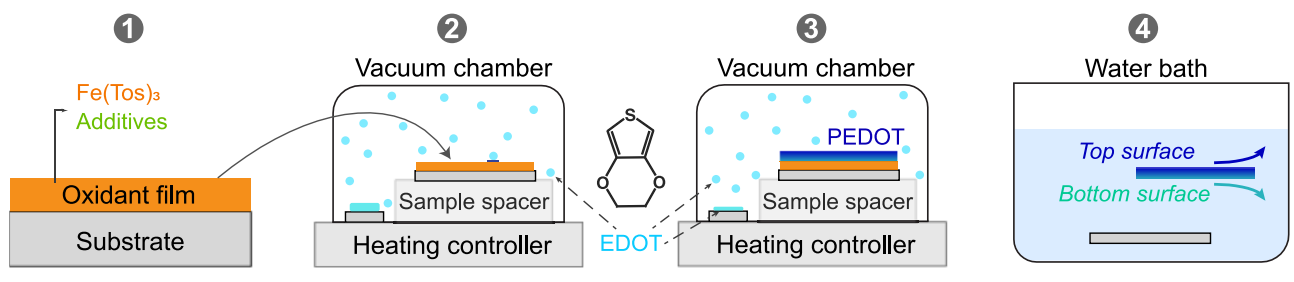

Figure 5.2 | Typical procedure of VPP. Oxidant solution is first spin coated on the substrate and transferred into a vacuum oven filled with monomers. After certain time of reaction, the sample is moved out and washed by ethanol bath. For free-standing films, we used a water bath for washing. The figure is reproduced from Reference 25 with permission from the Royal Society of Chemistry.

The obtained polymer film is formed on top of the oxidant ${ }^{26,27}$ and a washing step is required to remove these unwanted materials (e.g., unreacted and excessive oxidants) and byproducts in the films. We usually immerse the whole sample into an ethanol bath to rinse it, followed by drying with a mild nitrogen flow. We repeat the above process multiple times until we could see a homogeneous reflective colour from the sample surface, which implies that the byproducts have been completely removed.

In the washing step, we can detach the film from the substrate by utilizing a mechanical perturbation to the bath (step 4). This detaching effect can be improved using a water bath instead of an ethanol bath and using silicon wafers instead of glass substrates ${ }^{25,28}$. The free-standing film can be re-deposited onto new substrates guided by tweezers in the water bath. It is therefore possible to flip the film for separate investigation of top and bottom surfaces. We used this free-standing film transfer technique in Paper 2. It should be noted that the detaching process does not influence the film properties, because the obtained polymer film is always on the top of the oxidant film instead of the initial substrate and the standard washing step with ethanol bath also contains the process of film detaching and redepositing.

The conductivity of VPP PEDOT films can be further enhanced by acid treatments, such as sulfuric acid ${ }^{29}$, hydrobromic acid ${ }^{9}$, and other mineral acids ${ }^{30,31}$. The processing details for the acid treatment can be different ${ }^{32,33}$. In the research presented in this thesis, we soaked PEDOT films in concentrated sulfuric acid (1M to $3 \mathrm{M}$ ) for $10-15$ 
minutes, followed by a deionized water wash and a thermal annealing step at $100{ }^{\circ} \mathrm{C}$ for 10-15 minutes $^{34}$.

\subsubsection{Processing parameters for VPP}

In order to obtain polymers with high conductivity, we can tune and optimize the VPP processing parameters. In this thesis, we adopted optimized parameters from previous studies ${ }^{11,16,29}$. These processing parameters can be divided into four categories, i.e., oxidant recipe, substrate and its treatments, polymerization kinetics control, and posttreatments.

The earliest attempt of VPP for conducting polymers can be traced back to $2000 \mathrm{~s}^{35}$, when $\mathrm{FeCl}_{3} \cdot 6 \mathrm{H}_{2} \mathrm{O}$ was used as the oxidant and resulted films showed sheet resistance of $200 \mathrm{ohm} / \mathrm{sq}$ with thickness of $600 \mathrm{~nm}$. Follow-up research indicated that crystallites formed in the film ${ }^{36}$, which now is believed to be caused by oxidant crystallization ${ }^{37}$. Rational selection of oxidants thus plays a critical role in defining the final properties of the films ${ }^{38-41}$. Currently, iron(III) tosylate and iron(III) trifluoromethanesulfonate are two main iron salt oxidants for VPP. In addition to oxidants, additives can be added to control oxidant film morphology and to regulate polymerization kinetics in later steps. It was demonstrated that block-co-polymers were beneficial in preventing the abovementioned crystallization of oxidants and thus provided more uniform films $s^{2,17,37,42}$. The addition of a base, such as pyridine ${ }^{40}$ or imidazole ${ }^{1}$, was reported to be effective in controlling the polymerization kinetics, since the deprotonation initiated by base is one critical step for dimer and polymer formation (Figure 5.1). The solvent used to dissolve the oxidant and additive is normally alkanols, and previous studies demonstrated that VPP PEDOT using ethanol exhibited the highest conductivity ${ }^{11}$. Co-solvents could be useful in assisting the self-arrangement of the film morphology, similar to secondary doping ${ }^{11,16}$. However, the attempt of using N-methyl-2-pyrrolidone (NMP), which was widely used in $\mathrm{ICP}^{4}$, was rarely reported for VPP ${ }^{43}$. The amount of water in the oxidant recipe is another key factor and a recent study on iCP demonstrated that the accurate control of water enabled record high conductivity of above $6000 \mathrm{~S} / \mathrm{cm}$ in PEDOT thin films ${ }^{44}$. Common chemicals used in oxidant recipes are shown in Figure 5.3.

\section{Oxidants}

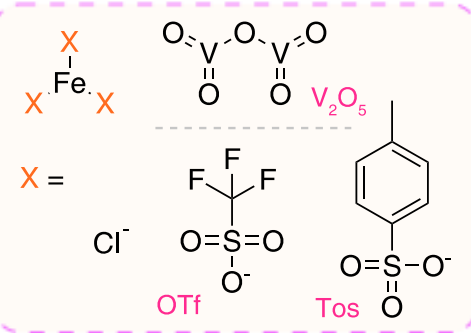

\section{Additives}

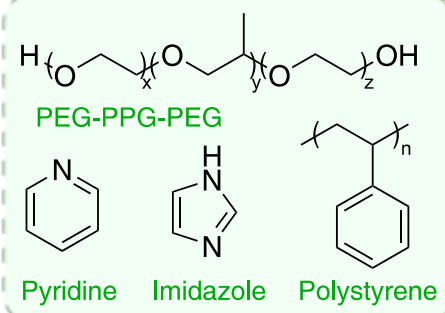

\section{Co-solvents}

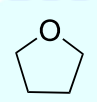

THF<smiles>CNC=O</smiles>

DMFI

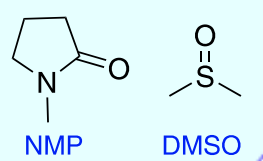

Figure 5.3 | Typical chemicals used for oxidant recipes. These chemicals include oxidants, additives, and co-solvents. 
There is no strict requirement regarding substrate for VPP, but the properties of the substrate could potentially affect the final performance of the polymer ${ }^{45}$. This effect can clearly be seen in previously mentioned free-standing film transfer technique, where PEDOT:Tos films on silicon wafers with hydrophobic surface showed poorer adhesion than films on glass substrates ${ }^{25}$. Recent studies showed that utilizing EDOT grafted surfaces can improve both conductivity and film adhesion ${ }^{46}$. Similarly, PEDOT deposited on top of another PEDOT layer (layer-by-layer method) showed an abrupt increase in conductivity, further demonstrating the importance of substrate choices ${ }^{28,47}$. Stretchable substrates can shape the form of the PEDOT layer by multiple interactions (e.g., cross-linking) and enable a balance between stretchability and conductivity $28,48,49$. Substrates made of functional materials, such as polyvinylpyrrolidone (PVP) fibers ${ }^{50}$ and carbon fibers ${ }^{15}$, exhibited great capability in shaping the resulting nanostructures of conducting polymers for energy storage and biomedical applications ${ }^{21}$. Similarly, well-defined templates on substrates can provide spatial confinement for the growth of polymers into desired micro/nano patterns ${ }^{22,23,51}$.
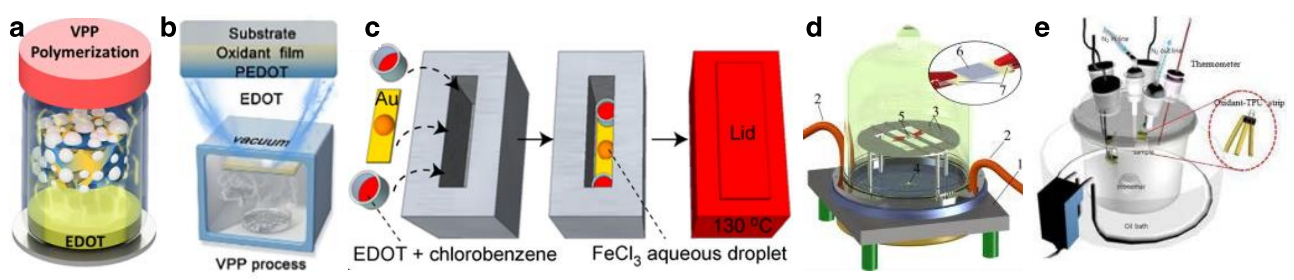

Figure 5.4 | Typical set-ups of VPP chambers reported in literatures. a, Desiccator. b, Oven. c, Tube furnace. $\mathbf{d}$ and $\mathbf{e}$, self-built chambers with heat controllers and gas inlets/outlets. The figures are reprinted from Reference 15, 21, 48, 52, and 53 with permission from the American Chemical Society, John Wiley and Sons, and Elsevier.

The polymerization kinetics for VPP can be controlled through various parameters. Firstly, the reaction can be affected by the heating control of the chamber, including the temperature of the crucible or holder for monomers, the sample substrates, and the environment inside the chamber. The chamber set-up also differs between different labs and reports, from desiccators ${ }^{29,38,39,54,55}$ and vacuum ovens $2,11,15,16,18$ to self-built chambers equipped with multiple heat controllers and gas inlets/outlets $27,48,53$. This leads to different levels of control precision (Figure 5.4). A low temperature is usually preferred in regulating the growth rate of the film ${ }^{29}$, and an interesting study on iCP showed that conducting polymers deposited on ice substrates showed relatively high degrees of crystallinity and excellent conductivities ${ }^{56}$. Secondly, the vacuum pressure is another critical parameter that was widely discussed for VPP. Atmospheric environment had been demonstrated sufficient for polymer growth ${ }^{40,57}$, while lowering the pressure with vacuum pumps was believed to provide good reproducibility by negating variations in the lab environment conditions and avoiding inhomogeneous distribution of monomers inside the chamber ${ }^{58}$. In addition, VPP at low pressure can largely reduce the amount of water vapour in the chamber and therefore enhance the conductivity of the final films ${ }^{2}$. A recent study on iCP reported that the variations in the 
humidity of the lab environment induced drastic changes in polymer properties across different seasons ${ }^{44}$, which was also considered to be crucial for VPP. Lastly, the additives in the oxidant can affect the polymerization as we described above. Bases ${ }^{1,40}$, water $2,44,59$, block-co-polymers ${ }^{2,17,57}$, co-solvents ${ }^{11,48}$, and nanoparticles ${ }^{23,60}$ can tune the conducting polymer properties a lot, depending on their ratios, molecular weights, and $\mathrm{pH}$ values.

The post-treatment is the final possibility to alter the properties of VPP polymers. Postbaking is sometimes employed to improve the mechanical properties (e.g., robustness) of films ${ }^{61}$, although this step may induce some additional polymerization ${ }^{62}$. Post-baking is optional for VPP and for applications requiring high conductivities, this step can be skipped. Other post-treatments, including further doping or de-doping by chemicals, could also influence the polymer film properties. The physical states of the dopants, temperature, dopant concentration, treatment conditions are critical. Approaches and influences of post-treatments were summarized by several reviews ${ }^{30,63}$.

\subsubsection{UV-treatment of oxidants in VPP}

A UV-patterning approach was proposed by Edberg et al. ${ }^{61}$ and utilized to fabricate electrochromic devices 55,64 and electrical circuits (Figure 5.5) ${ }^{61}$. In brief, a UV exposure step of the oxidant is applied prior to VPP. The UV exposure can lead to changes in the oxidant, with effects also being dependent on the concentration of the block copolymer in the oxidant.
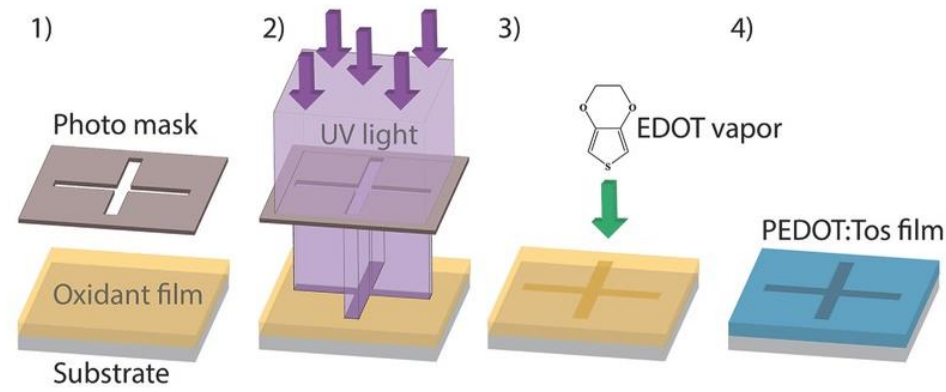

Figure 5.5 | Process flow of UV-treatment for VPP. The figure is reprinted from Reference 61 with permission from John Wiley and Sons.

It was found that UV exposure induces changes in the coordi-nation between iron, the counterion (Tos in that study), and water61. Possible mechan-ism of the UV-induced changes could be that UV light weakens the coordination effect of iron atom in the ethylene oxide moiety of the PEG-PPG-PEG ${ }^{16,43}$. With weaker coordination effect, iron salts exhibit higher reactivity for the polymerization, leading to a thicker film but poorer conductivity (probably due to shorter polymer chains) ${ }^{61}$. It should be noted that up to now there is no consensus on the physical or chemical mechanism of the UV treatment and further investigation is still needed. In this thesis, we studied and utilized UV- 
treatment as an effective approach to alter both optical properties and thickness of VPP PEDOT to generate structural colours (Paper 4).

\subsection{Colloidal lithography}

Plasmonic systems are mostly based on nanostructures and there are many methods to fabricate nanostructures by either top-down or bottom-up approaches ${ }^{65}$. Templateassisted growth and self-assembly of molecules or particles ${ }^{66,67}$ are two representative bottom-up techniques. For top-down methods, electron beam lithography, focused ion beam milling, and photolithography are broadly used by researchers to create patterns from nanoscale to microscale. In this thesis, we chose colloidal monolayer lithography, or colloidal lithography, as the main approach to structure polymers, since it is a welldeveloped technique with the possibility for large-scale production of nanostructured surfaces at low $\operatorname{cost}^{68}$.

\subsubsection{Basic principles}

Colloidal lithography is a technique combining the advantage of both bottom-up (selfassembly mask) and top-down approaches (etching or deposition for nanostructures). Two main steps are included in the method.

In the first step, a suspension of monodisperse colloids [e.g., polystyrene (PS) or silica] is deposited on top of a substrate with pre-treatments. Pre-treatments could adjust surface properties of the substrates and improve the arranging order of the colloids. A typically used one is depositing a monolayer of polyelectrolytes with positive charges (while the colloids carry negative charges) ${ }^{69}$. Colloids can then self-assembled on the surface during a suspension immersion step. With water rinsing, weakly attached or unattached colloids would be removed, leaving a short-range order (SRO) pattern as a mask for later steps (Figure 5.6).

In the second step, materials of interest can be deposited through the mask by thermal evaporation ${ }^{70}$ or other deposition methods (e.g., sputtering ${ }^{71}$ and electroplating ${ }^{72}$ ). The colloids can then be removed by a cleanroom tape or mild sonication ${ }^{73}$ and a nanohole array pattern could be transferred onto the materials (Figure 5.6a). Nanodisk array pattern is also possible to create with several additional steps, including a sacrificial layer and etching (Figure 5.6b), which is known as hole-mask colloidal lithography ${ }^{74}$.

In this thesis, we made nanodisks of conducting polymers by a modified version of colloidal lithography, where we deposited a thin sacrificial (protection) layer [e.g., poly (methyl methacrylate) or PMMA] on top of conducting polymer thin films, and used the self-organized PS colloids as a mask for reactive ion etching (RIE) ${ }^{34}$. By acetone soaking together with mild ultrasonication treatments, colloids attached to PMMA could be removed, resulting in SRO nanodisk arrays of conducting polymers (Figure 5.6c). 

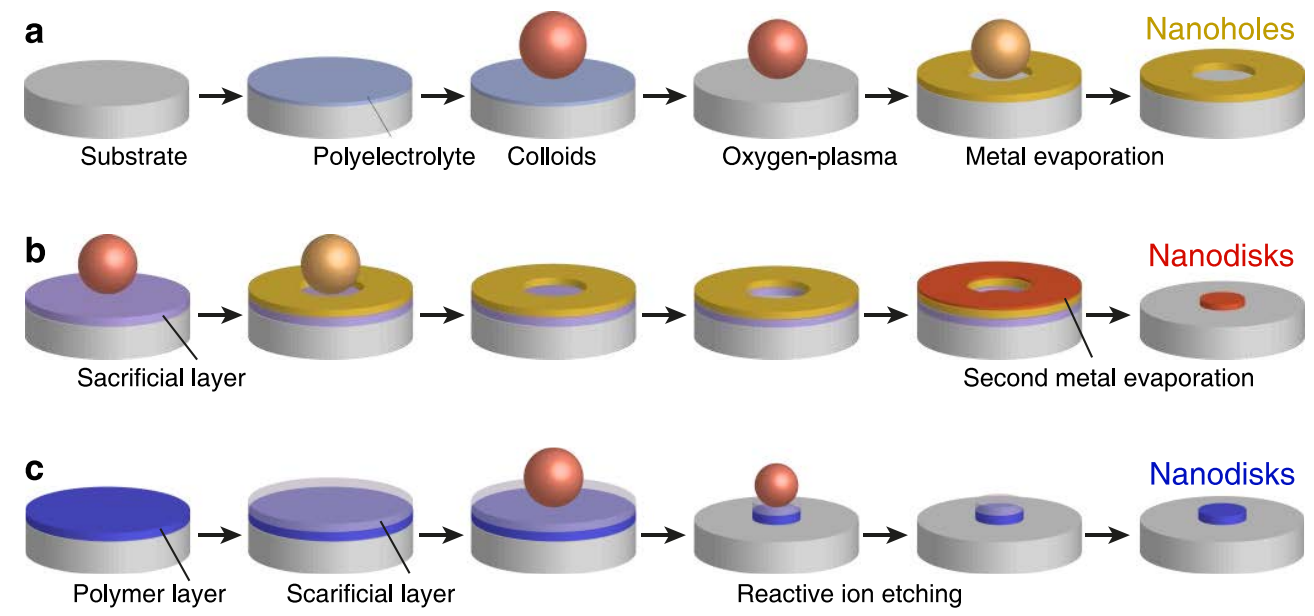

Figure 5.6 | The process flow for standard colloidal lithography. a, The standard procedure for nanoholes. b, Hole-mask method for nanodisks. c, Modified procedure for nanodisks.

Although large-scale patterns can be prepared in a cost-effective method, the colloidal lithography is challenged by the limited numbers of available patterns. For plasmonic applications requiring arbitrary nanostructures, electron beam lithography or focused ion beam milling are still the best choices.

\subsubsection{Processing parameters}

Since the self-assembled colloids are used as the mask for colloidal lithography, the arrangement (coverage or inter-distance) of colloids plays a critical role in determining the finally obtained nanostructures. Some key parameters are introduced below.

The colloids can be deposited onto the substrate by different approaches, such as dip coating $^{70,75}$, spin coating ${ }^{76}$, solvent evaporation ${ }^{77}$, and electrophoretic deposition ${ }^{78}$. We made masks using separated PS colloids arranged in a SRO ${ }^{69,70,73}$ instead of closely packed colloid arrays with periodicity ${ }^{79}$. To create such patterns, we first positively charge the substrate by its adsorption of polyelectrolytes. PS colloids with negative charges can easily be adsorbed on the substrate and a constant deionized water flow could easily remove colloids with poor adhesion (e.g., accumulating on top of other colloids), leaving a monolayer of colloids. The inter-distance between the colloids can be altered by varying their electrostatic repulsion using salts (e.g., sodium chloride) in the colloid suspension 68,75 .

In this thesis, we utilized PS colloids with diameters ranging from 100 nanometers to 1 micrometer to create self-assembled masks ${ }^{34}$. The self-organization time for small colloids (diameter below $200 \mathrm{~nm}$ ) to reach thermodynamical equilibrium was normally one to several minutes $69,70,73$ while for large particles, a much longer time scale was required (e.g., 15 to 60 minutes) since large colloids diffused more slowly through the same electrostatic force compared to small particles. The time could probably be redu- 
ced by mild heating or application of an external electric field. Large colloids also have another issue that they tend to form aggregates even in the suspension and ultrasonicator or vortex mixer could be of help in reducing the agglomeration.

The adhesion of the colloids to the substrate can be enhanced by applying fuming liquid on the colloids prior to the water flow rinsing step ${ }^{80}$. Fuming liquid can be fuminghot ethylene glycol (EG) or propylene glycol (PG). An additional thermal annealing step (for several minutes at around $110^{\circ} \mathrm{C}^{80}$ ) after rinsing and drying could further deform the colloid shape into hemispheres and provide better adhesion. These steps are optional for small colloids but vital for the self-assembly of large-diameter particles.

In the preparation of nanodisks using the procedure in Figure 5.6c, the reactive ion etching step can also affect the final size of the nanodisks. Because PS and PEDOT are organics and share similar etching rate, the colloids shrink during the removal of the uncovered PEDOT layer, resulting in smaller diameters for the final nanodisks than expected only based on the colloid size. Optimization of etching power and etching time could reduce this effect to some extent.

\subsection{Spectroscopic ellipsometry}

Spectroscopic ellipsometry is an optical characterization technique for the determination of dielectric properties and thicknesses in layered sample structures. It measures the relative polarization changes of reflected light from (or transmitted light through) the sample surface and offers information about the samples via a model fitting process. Compared with standard reflectivity ${ }^{81,82}$ or absorption methods ${ }^{83}$ for optical parameter extraction, spectroscopic ellipsometry places no requirements of KramersKronig transformation and associated hypothesis for frequency ranges beyond the measurements. In addition, information on thin film anisotropy can be obtained from ellipsometry measurements ${ }^{6,84}$.

\subsubsection{Working principles}

Ellipsometry is a polarization-based characterization method. The polarization of light describes the "shape" of the electric field evolving during the light propagation. In Figure 2.5, we defined the $s$ - and $p$-polarization of light, and their reflection changes at the surface are described by the Fresnel Equations (2.18). For the interface between two optically isotropic materials, the Fresnel coefficients can be expressed by ${ }^{85}$

$$
\begin{aligned}
& r_{s}=\frac{n_{1} \cos \theta_{1}-n_{2} \cos \theta_{2}}{n_{1} \cos \theta_{1}+n_{2} \cos \theta_{2}}=\left(\frac{\mathbf{E}_{\mathbf{r}}}{\mathbf{E}_{\mathbf{i}}}\right)_{s}, \\
& r_{p}=\frac{n_{1} \cos \theta_{2}-n_{2} \cos \theta_{1}}{n_{1} \cos \theta_{2}+n_{2} \cos \theta_{1}}=\left(\frac{\mathbf{E}_{\mathbf{r}}}{\mathbf{E}_{\mathbf{i}}}\right)_{p},
\end{aligned}
$$

where $n_{1}$ and $n_{2}$ are the complex indices of refraction in the materials before and after the interface, and $\theta_{1}$ and $\theta_{2}$ are the corresponding angles of light propagation with respect to the sample normal. $\mathbf{E}_{\mathbf{r}}$ and $\mathbf{E}_{\mathbf{i}}$ are the electric fields (including amplitude and 
phase) for reflected and incident light. The ratio of the two quantities, $\rho$, can be calculated as 85

$$
\rho=\frac{r_{p}}{r_{s}}=\frac{\left|\mathbf{E}_{\mathbf{r}, \mathbf{p}}\right| /\left|\mathbf{E}_{\mathbf{i}, \mathbf{p}}\right|}{\left|\mathbf{E}_{\mathbf{r}, \mathbf{s}}\right| /\left|\mathbf{E}_{\mathbf{i}, s}\right|} e^{i\left(\delta_{p}-\delta_{s}\right)}=\tan (\psi) e^{i \Delta},
$$

where $\psi$ and $\Delta$ are two new quantities that are the basic parameters that we measure in ellipsometry, and they do not contain certain physical meaning ${ }^{85}$. For a two-layer system (e.g., air-material), the permittivity of the whole system can be calculated by ${ }^{85}$

$$
\varepsilon=\sin ^{2} \theta\left[1+\tan ^{2} \theta\left(\frac{1-\rho}{1+\rho}\right)^{2}\right]
$$

where $\theta$ is the incident angle of the probing light. However, in practice, the target systems normally have at least 3 layers (the material of interest has finite thickness and therefore the system becomes air-material-air or air-material-substrate) and the transfer matrix method and numerical calculations have to be used for further analysis.

Instead of $\psi$ and $\Delta$, there are other types of the ellipsometric data, such as the NCSnotations: ${ }^{86}$

$$
\begin{gathered}
N=\cos (2 \psi), \\
C=\sin (2 \psi) \cos (\Delta), \\
S=\sin (2 \psi) \cos (\Delta),
\end{gathered}
$$

where $N, C$, and $S$ are the measured quantities in modern ellipsometers ${ }^{86}$.

Ellipsometry is a non-contact and therefore a non-destructive method and can probe ultrathin films of only a few angstroms with high precision ${ }^{87}$. However, ellipsometry is not a direct characterization method, physical properties typically cannot be directly extracted from raw data. Thus, a sophisticated data analysis process is required. The spatial resolution of ellipsometry is not as good as microscopy-based techniques.

\subsubsection{Components of an ellipsometer}

A typical ellipsometer contains six main components, as shown in Figure 5.7.

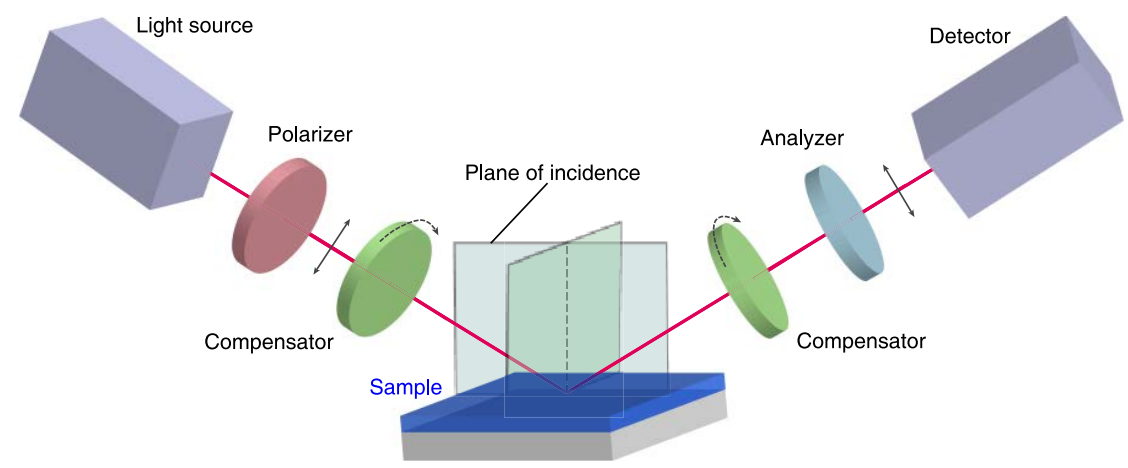

Figure 5.7 | The set-up of a spectroscopic ellipsometer. Six main components are shown: light source, polarizer, compensators, analyzer, and detector. 
Since ellipsometry measures polarization, polarizers are the core elements of the equipment. They are placed in front of the light source to produce a well-defined polarized state and in front of the detector to characterize polarization changes (as an analyzer). In the setup, the compensator, usually a waveplate or a retarder, is used to introduce phase delay between orthogonal polarizations. With a compensator, linearly polarized light can be converted into circularly or elliptically polarized light (see Figure 2.2). The light source and detector define the wavelength (frequency) range of the measurement.

In this thesis, we used $\mathrm{RC}^{\circledR}{ }^{\circledR}$ and IR-WASE ${ }^{\circledR}$ manufactured by J.A. Woollam Company for the ellipsometric measurement from UV $(210 \mathrm{~nm}$ ) to MIR (around $30 \mu \mathrm{m})$. For the FIR spectral range, we used a custom-built Fourier transform-based FIR ellipsometer at University of Nebraska, Lincoln ${ }^{88}$. For the $\mathrm{THz}$ spectral range (100 GHz to $\left.1 \mathrm{THz}\right)$, we utilized a custom-built terahertz frequency-domain ellipsometer at the Terahertz Materials Analysis Center (THeMAC) at Linköping University ${ }^{89}$.

\subsubsection{Data analysis}

Since the interpretation of ellipsometric raw data is not intuitive in most cases, it is necessary to perform data analysis to be able to extract physically relevant parameters from the experimental data. The data analysis is a repeating process of stratified layer model construction and refining (or optimization) as illustrated in Figure 5.8.

In the first step, we build an optical model for the whole system based on assumed basic properties of the materials in each layer (see Section 2.3). The optical model for each layer contains a certain number of parameters that can be tuned in the later fitting steps. For instance, in the Drude model for metals, the parameters could be charge density $(n)$ and mobility $(\mu)$ or plasma frequency $\left(\omega_{p}\right)$ and relaxation time $(\tau)$, as discussed in Section 2.3.3. For materials with known properties, tabulated list of material's optical parameters (refractive index or permittivity) could also be used, although the properties of this layer will then be fixed without fitting parameters.

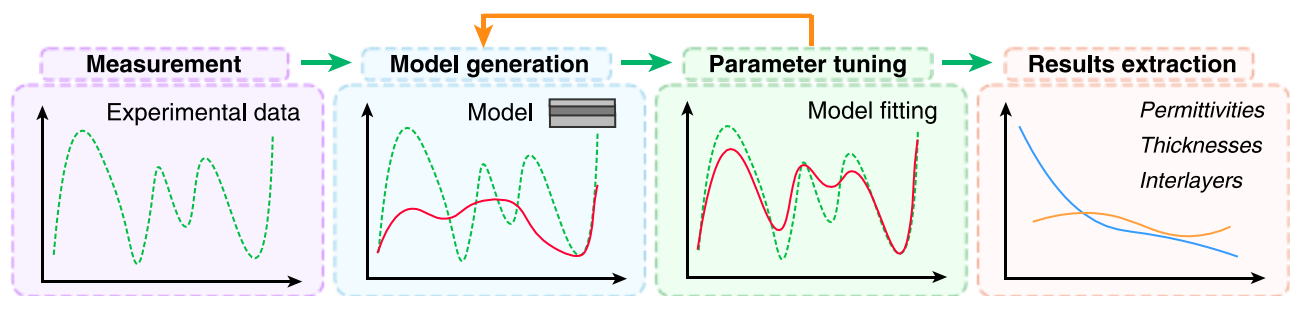

Figure 5.8 | The procedure for ellipsometric data analysis. The data analysis is based on the repeating process of model generation and parameter tuning until the model generated data fits as close as possible to the experimental data (low mean-squared error).

In the second step, we input initial "guess" values of all parameters in the model of the system, and calculate model-generated ellipsometry data. Normally, large differences 
exist between the initial model-generated and the experimental curves (see Figure 5.8). Thus, a fitting step to optimize model parameters is necessary.

An indicator that describes the difference between model and experimental curves is the mean-squared error (MSE): 87

$$
M S E=\sqrt{\frac{1}{2 N-M} \sum_{i=1}^{N}\left[\left(\frac{\psi_{i}^{\text {mod }}-\psi_{i}^{\text {exp }}}{\sigma_{\psi, i}^{\text {exp }}}\right)^{2}+\left(\frac{\Delta_{i}^{\text {mod }}-\Delta_{i}^{\text {exp }}}{\sigma_{\Delta, i}^{\text {exp }}}\right)^{2}\right]},
$$

where $N$ is the number of data points (given by the number of wavelengths, angles of incidence, etc.) in the measurement, $M$ is the number of fit parameters, $\sigma$ is the standard deviations of the experimental uncertainty. In standard ellipsometry analysis software, i.e., WVASE ${ }^{\circledR}$, regression analysis algorithms are applied to optimize the fitting to reach the minimum MSE for the used model.

In some cases, additional modification of the model might be necessary, such as the introduction of interface layers, surface roughness layers, layer property gradient, or optical anisotropy for certain layers.

\subsubsection{THz and FIR ellipsometers}

$\mathrm{THz}$ and FIR frequency ranges with low energies are sensitive to charge carriers and are therefore useful for the characterization of charge carrier transport properties of conducting polymers. Ellipsometry measurement in an ultrawide spectral range can be beneficial in determining the optical parameters of materials with high precision and accuracy. Such measurements can reduce the uncertainties at low frequencies which are sensitive to phonons (or molecular vibrations) and localized charge carriers ${ }^{54,90}$.

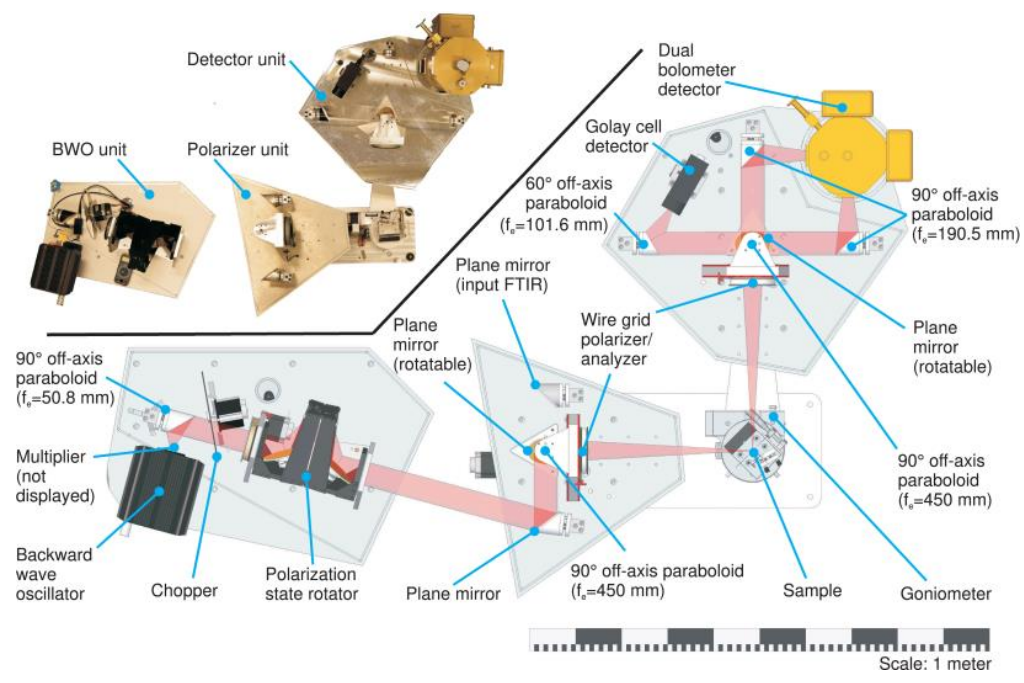

Figure 5.9 | The set-up of a THz frequency domain spectroscopic ellipsometer. The figure is reprinted from Reference 89 with permission from IEEE. 
In the THz ellipsometer at Linköping University, a backward wave oscillator is used as the light source (Figure 5.9). With Schottky diode multipliers, the available spectral range is $100 \mathrm{GHz}$ to $1 \mathrm{THz}$, filling the frequency gap between conventional optical measurements and AC current characterizations (with method of impedance). In addition, potential accessories such as a temperature-control stage and a flow cell can be augmented, providing further information on the material properties including the temperature-dependence and redox state-dependence of charge carrier transport in conducting polymers.

\subsection{Optical characterization and simulation}

Optical characterization and simulations are key parts for the study of conducting polymer thin film optics and nano-optics. In this thesis, we used standard extinction spectroscopy and the finite-difference time-domain method for simulation.

\subsubsection{UV-Vis-NIR spectrometer}

A UV-Vis-NIR spectrometer is an instrument for transmission or extinction measurement of samples although in most cases it is named as absorption spectrometer. It is widely used to characterize conducting polymers and can provide information on film thickness ${ }^{29}$, doping states ${ }^{91}$, and possible optical transitions ${ }^{92}$ (e.g., energy gaps). A typical set-up for a UV-Vis-NIR spectrometer is shown in Figure 5.10.

The most commonly used UV-Vis-NIR spectrometer in this thesis utilizes a dispersive method to measure samples. In such spectrometers, a dispersion device (e.g., prism) is used to separate the white light from the source into monochromatic light, which is scanned across different wavelengths during measurement. For each wavelength, light passes through a beam splitter and is divided into two beams for transmission measurements of reference and sample. The obtained spectra can be interpreted in terms of transmission (Equation (2.11)) or absorbance (Equation (2.13)).

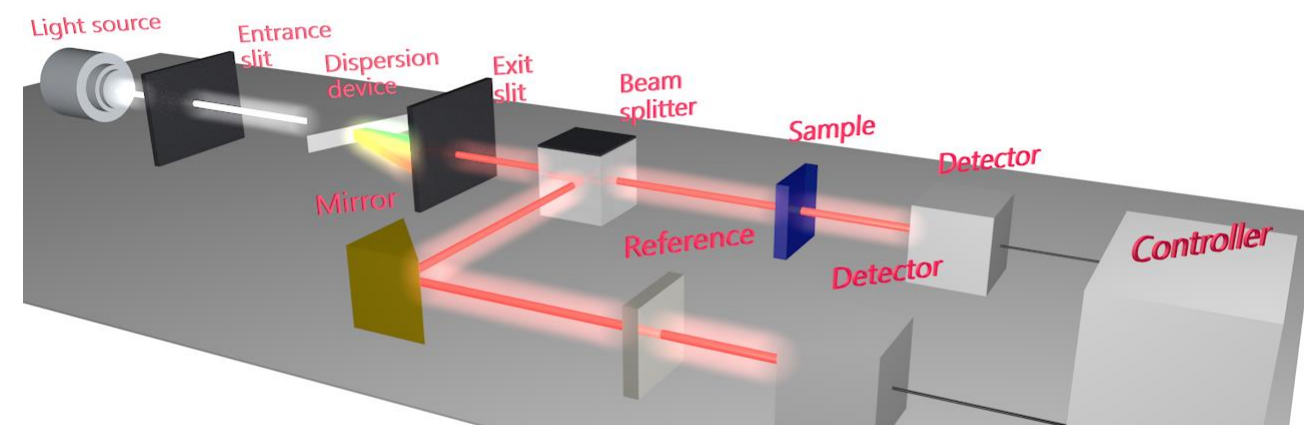

Figure 5.10 | The set-up of a UV-Vis-NIR spectrometer. The incident beam is split into two beams to measure both sample and reference and collected by detectors, respectively. 
Extinction spectra used in our study could provide information on the redox-state of the conducting polymers for in operando measurements (Section 5.6.5), where we could clearly observe fingerprints of polaron bands, bipolaron bands, and neutral state absorption (band gap) of the polymer ${ }^{63}$. In this thesis, we used an absorption spectrometer model Lambda 900 manufactured by PerkinElmer.

\subsubsection{Fourier Transform Infrared spectrometer}

Different from the dispersive methods normally utilized for UV-Vis-NIR spectrometer, Fourier transform infrared (FTIR) spectrometer collects all data simultaneously via interferogram and converts them into an infrared extinction spectrum. The instrument is based on the Michelson interferometer (Figure 5.11).

In the interferometer, the light beam from the source is divided into two sub-beams by a beam splitter. One light beam is guided to a stationary mirror with a fixed position while the other light beam is heading to a movable mirror whose position is precisely controlled by a linear translation stage. When the reflected beams from the two mirrors return back to the beam splitter, the difference in their optical paths could produce interference. By collecting data of the interference intensities at varying optical path differences, we obtain the interferogram. With the algorithm of Fourier transformation, the data can be converted into a standard IR spectrum in frequencies (Figure 5.11).
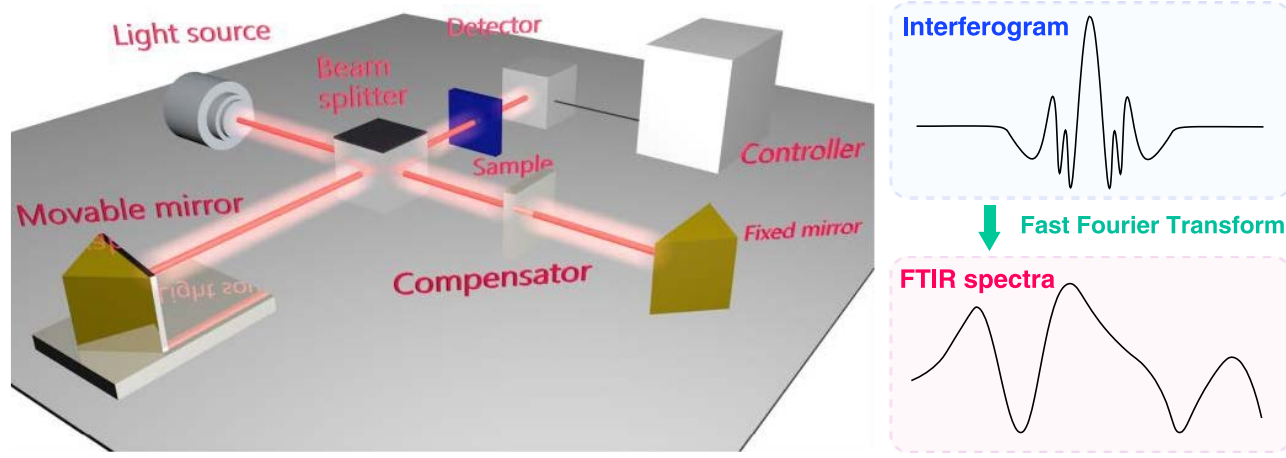

Figure 5.11 | The set-up of a FTIR spectrometer. The interferometer utilizes a movable mirror to control the optical path differences to generate different interference patterns. The obtained interferogram can be interpretated into spectra in wavenumber by Fourier transformation.

FTIR is a fast measurement method and offers precise characterization of samples with high resolution. Various accessories for FTIR are available to meet the measuring requirements of different samples. For example, the attenuated total reflection set-up can be used for thick opaque films and liquid samples which are not preferrable for standard transmission measurement.

FTIR measurement is normally carried out in a chamber with purging nitrogen. However, it may still be difficult to avoid the appearance of water vapour and carbon dioxide 
signals in the obtained spectra. In our measurements ${ }^{34,54}$, we observed signals of water vapour in the range $4000-3400 \mathrm{~cm}^{-1}$ and carbon dioxide around $2400 \mathrm{~cm}^{-1}$.

FTIR is often used to identify functional groups in organic materials and collecting covalent bonding information. In this thesis, we used FTIR to measure the NIR and MIR plasmonic resonance signals of conducting polymer nanostructures (Paper 3). The equipment we used was a FTIR spectrometer model Equinox 55 manufactured by Bruker.

\subsubsection{Angle-resolved reflection measurements}

To measure angle-dependent reflection, we used a custom-built set-up based on two concentric goniometers. The light detector is placed on the outer wheel while the sample is placed on the inner wheel.

For angle-resolved light scattering measurements, we normally fixed the incident angle at $0^{\circ}$ (normal to the sample surface) and alter the angle between detector and normal
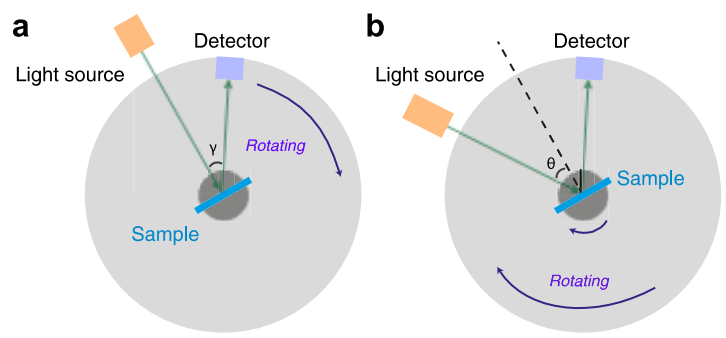

Figure 5.12 | Angle-dependence measurements. a, Scattered reflection. $\mathbf{b}$, Specular reflection.

$(\gamma)$ to collect scattered light at different angles (Figure 5.12a). For specular reflection measurements, we varied the incident angle from $10^{\circ}$ to $60^{\circ}$ and collected its specular reflected light (Figure 5.12b). The spectrometer we used was an Andor Shamrock 303i, equipped with a Newton CCD detector ${ }^{93}$.

\subsubsection{Optical microspectroscopy}

To record reflectance spectra from small areas, we used optical microspectroscopy as illustrated in Figure 5.13. In the set-up, a fiber spectrometer is connected to an optical microscope via a beam splitter, which could separate the reflected light into two paths, one to a camera and the other to a fiber-coupled spectrometer. With this set-up, it is possible to reduce the active probing area down to the scale of a few micrometers and simultaneously record both reflection spectra and microscopic images ${ }^{93,94}$.

In our studies, we used a Nikon Eclipse L200N optical microscope and a fiber spectrometer from Ocean Optics (QePro). The minimal spot size for optical measurements depends on the diameter of the optical fiber and can be as small as a few micrometers. Electrochemical flow cell

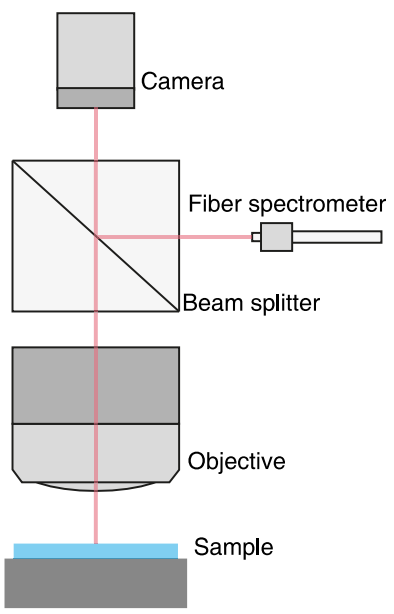

Figure 5.13 | The set-up of a microspectroscopy. 
can also be implemented for in operando measurements (Section 5.6 and Paper 4).

\subsubsection{Finite-difference time-domain simulation}

The finite-difference time-domain (FDTD) method is a numerical simulation approach for nanophotonic devices and systems. The software allows us to predict optical behavior, and to visualize the optical near-field distribution of nanostructures.

In this method, we break up the simulation region, i.e., (nano-)structures, into small sub-units (grid cell) and Maxwell's equations are applied for each grid cell. A short pulse of electromagnetic wave is used for the simulation and the collected response will be converted via Fourier transformation into spectra over a range of wavelengths. By using different types of light sources, boundary conditions, and monitors, we could obtain detailed information of the system. The precision and accuracy depend on the mesh size of the grid cell and fitting quality of materials' optical parameters.

In this thesis, we used the commercial software Lumerical FDTD solutions for optical simulations of nanodisk arrays, single nanodisks, and conducting polymer thin films (see Paper 3 and Paper 4).

\subsection{Microstructural characterization}

The microstructures of thin films and nanostructures can be characterized by various techniques, including atomic force microscopy, x-ray diffraction spectroscopy, and scanning electron microscopy. In this thesis, we focus on the most useful methods that can provide topological information of nanostructures and crystallinity of polymer thin films.

\subsubsection{Atomic force microscopy}

The atomic force microscope (AFM) is a type of scanning probe microscope that is used to characterize the height (topology) and phase (morphology) information of thin films. The working mechanism of AFM is based on the measurement of repulsive or attractive force between the specimen surface and a small tip attached on a cantilever. The AFM provides high resolution imaging for both lateral and vertical directions of the sample, to the order of nanometers. Normally, three working modes are available for AFM: contact, non-contact, and tapping (or intermittent) modes. In this thesis, we only used tapping mode, where the tip is oscillating at its resonance frequency near the specimen surface. Compared with scanning electron microscopy, AFM has no requirement on electrical conductivity of the sample and thus would be more convenient for the characterization of isolated nanostructures and insulating materials.

A typical AFM set-up is illustrated in Figure 5.14, where a tiny probing tip is placed on a cantilever and scanned over the sample surface. The interaction between specimen 
surface and tip might cause changes in either amplitude or phase of the tip oscillation, and through certain transformation algorithm, height or phase image of the sample could be generated (right part of Figure 5.14).

In this thesis, we used AFM to characterize the surface morphology and topology of PEDOT:Tos films (Paper 2) and the distribution of nanodisks made of PEDOT:Sulf (Paper 3) ${ }^{25,34}$. The AFM system we used was a Bruker Veeco Dimension 3100 and AFM data analysis was carried out on NanoScope provided by Bruker.
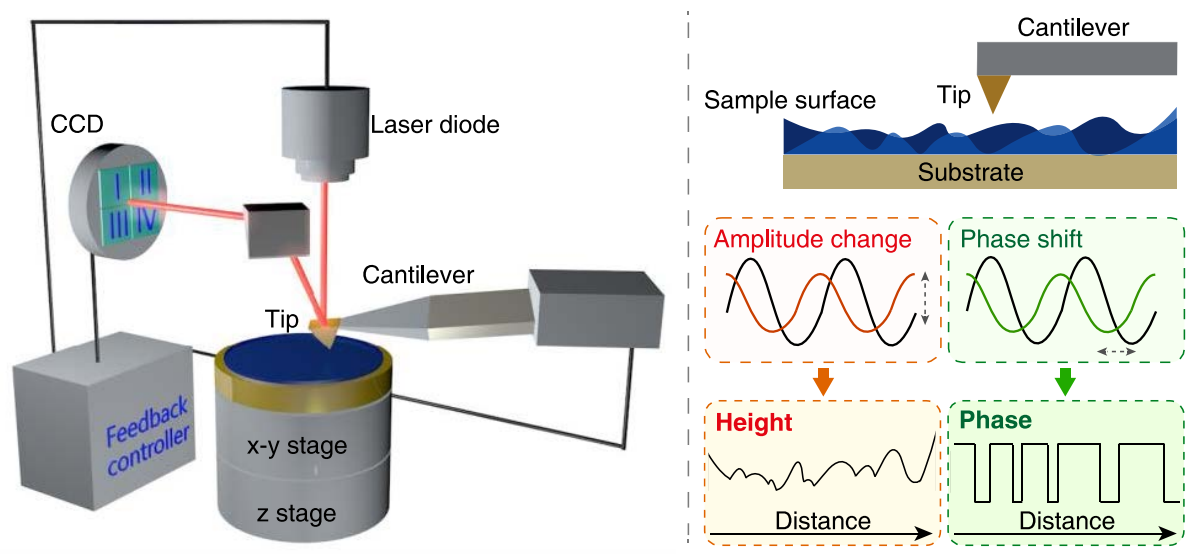

Figure 5.14 | The set-up of an AFM and its working principles. The left part schematically exhibits the structure of AFM: the tip on the cantilever is controlled by a feedback system.

\subsubsection{Grazing incidence wide-angle X-ray scattering}

X-ray diffraction and scattering analysis provide rich information on the microstructure of polymer materials. In such measurements (Figure 5.15), a beam of x-ray irradiates the sample surface at a grazing incident angle $\left(\alpha_{i}\right)$ and the intensities of scattered light are collected in a two-dimensional way (characterized by exit angle $\alpha_{f}$ and out-of-plane angle $\psi$ ). Depending on the sample-detector distance, we can obtain either wide-angle (GIWAXS) or small-angle scattering (GISAXS) spectra95. The length scale in GISAXS is from a few to several hundred nanometers, useful for probing the nanostructure of thin films. GIWAXS, which we used in this thesis, is broadly used for investigation of microscale morphology, including the details of crystalline and amorphous domains formed in thin films ${ }^{95}$. With GIWAXS, we can obtain the crystal structure and the orientations of crystallites with respect to the substrate (and their corresponding fractions).

The obtained spectra present two-dimensional patterns in reciprocal-space and the spots of high scattering intensity indicate the crystal planes (or stacks) as shown in Figure 5.16. For PEDOT-based materials (see Section 4.3), two configurations of crystallite stacking can be directly identified from the pattern: face-on and edge-on ${ }^{96}$. To calculate their fractions, detailed analysis, such as integration over azimuthal angle, is required ${ }^{5,95}$. 


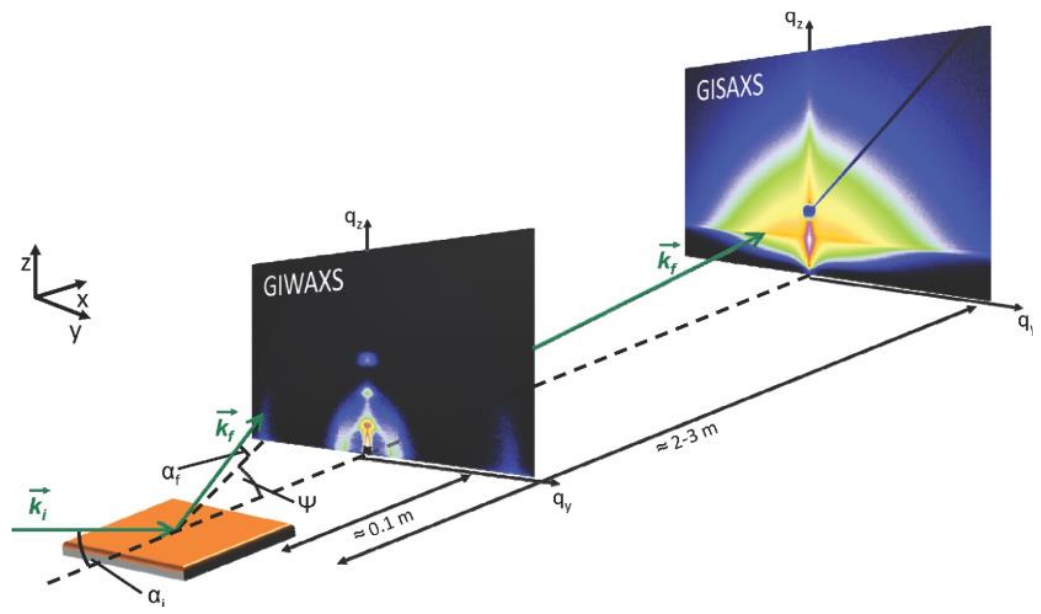

Figure 5.15 | Schematic illustration of x-ray grazing incident measurements. The Figure is reprinted from Reference 95 with permission from John Wiley and Sons.
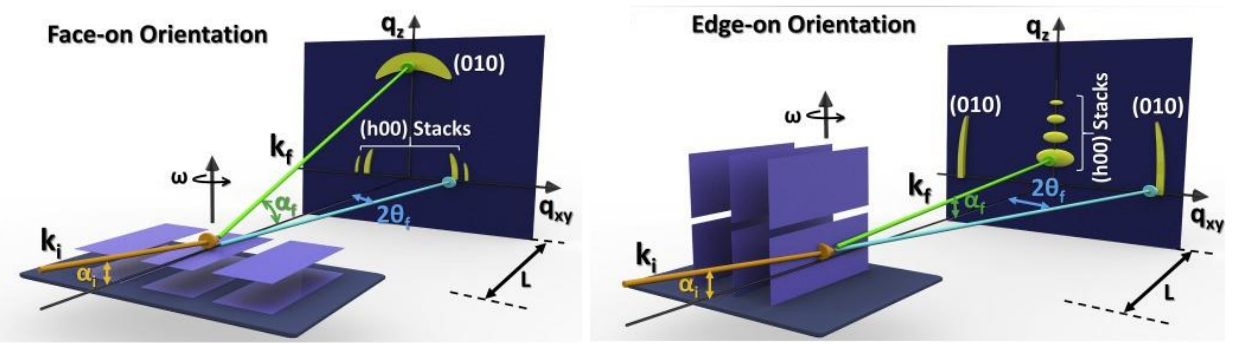

Figure 5.16 | Principles for GIWAXS measurements. The different orientations of crystallites can form different patterns. The figure is reproduced from Reference 96 under the terms of the Creative Commons CC-BY license.

In this thesis, GIWAXS measurements were performed on the Dutch-Belgian Beamline (DUBBLE CRG), station BM26B, at the European Synchrotron Radiation Facility (ESRF). PEDOT films were deposited on silicon wafers and GIWAXS was employed to probe the microstructures (mostly crystallite orientations) of the films with different incident angles to obtain information of the films at different depth ranges ${ }^{25}$ (Paper 2).

\subsection{Other basic characterization methods}

Besides optical and structural characterizations, other measurements on electrical and other properties were also indispensable for the research carried out in this thesis. Some of these are reviewed in the sections below. 


\subsubsection{Four-probe methods}

The electrical conductivity of thin films in the thesis was obtained by the four-probe method. The method utilizes four electrodes: two outer ones for current measurement and two inner ones for voltage measurement and the electrical resistance of the thin film can directly be calculated by Ohm's law:

$$
R=V / I \text {. }
$$

To determine the conductivity of the film, we need to include geometric factor for the certain configuration utilized for the measurement (Figure 5.17). For four-line probe configuration, where pre-patterned metal electrodes are below the thin film (shown in Figure 5.17), the electrical conductivity is ${ }^{97}$

$$
\sigma=\frac{s}{R w h} .
$$

where $s$ is the distance between two nearby electrodes, $w$ is the width of the film and $h$ is the thickness of the film, which can be determined using a stylus profiler (Section 5.6.2).

For the case of four-point probe configuration, four needles penetrate into the film by force and the conductivity can be calculated by ${ }^{97}$

$$
\sigma=\frac{\ln 2}{\pi} \frac{1}{R h}=\frac{1}{4.532} \frac{1}{R h} .
$$

Van der Pauw method, another type of four-probe measurement, can be used for films with irregular shapes ${ }^{98}$, which is beyond the scope of the thesis.
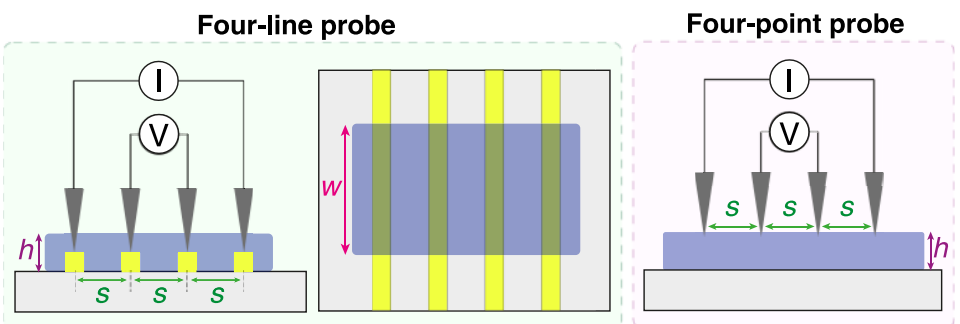

Van der Pauw method

Figure 5.17 | Typical set-ups for four-probe measurements. Three typical set-ups are shown: four-point probe, four-line probe, and Van der Pauw method.

In this thesis, we used the four-point probe set-up of a Signatone Pro4 S-302 resistivity stand combined with a Keithley 2400 source measure unit.

\subsubsection{Thickness measurements}

Thickness is a key parameter for calculating the electrical conductivity of polymer films. For polymer nanostructures, their thickness (height) was measured by AFM (Section 5.5.1). For thin films, we normally choose surface profilometer (stylus profiler) for direct 
thickness measurement and ellipsometry (Section 5.3) for verification ${ }^{54}$. The working principle of a surface profilometer is similar to the AFM in contact mode. A constant force is maintained between a stylus and the film while scanning over the film, and vertical variations of the stylus are recorded.

The surface profiler system we used in this thesis was a Veeco Dektak ${ }^{3} \mathrm{ST}$. The profiler is equipped with a diamond-tipped stylus and available for sample surface line scans. A scratch was made on the sample and the vertical difference between the surface and the trench gave the film thickness. The vertical resolution can reach a few nanometers.

\subsubsection{Contact angle measurements}

Contact angle measurement is used to determine the hydrophilicity or wettability of a surface. The angle is defined as the angle between the liquid and the solid phases in a solid-liquid-vapour three-phase system (Figure 5.18). The contact angle is affected by the surface energies of interfaces and described by the Young's equation:99

$$
\gamma_{S V}=\gamma_{S L}+\gamma_{L V} \cos \theta_{c},
$$

where $\gamma_{S V}, \gamma_{S L}$, and $\gamma_{L V}$ are the interfacial energy for solid-vapour, solid-liquid, and liquidvapour surfaces.

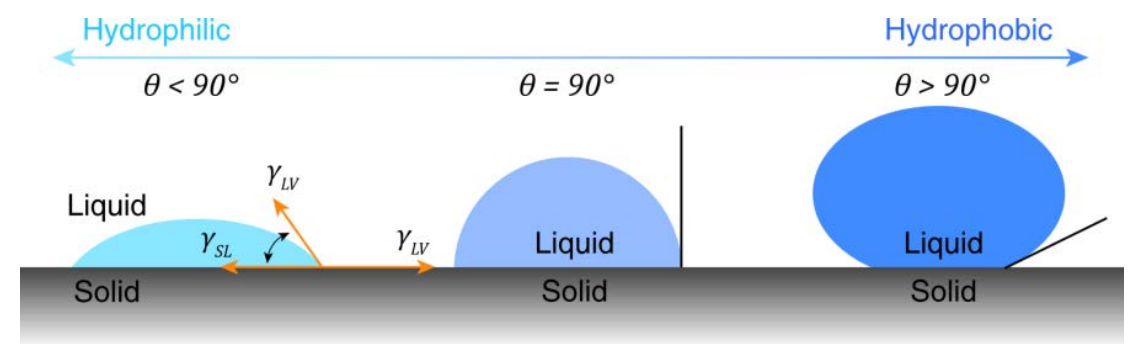

Figure 5.18 | The cross-section of droplets with different hydrophilicity.

A large contact angle indicates high surface energy and the surface is hydrophobic. In this thesis, we measured contact angles to demonstrate the hydrophilic surface of PEDOT which is favourable for the polymerization of EDOT monomers (Paper 2) ${ }^{25}$. The instrument we used was an optical contact angle and surface tension meter CAM200 manufactured by KSV Instruments.

\subsubsection{XPS and UPS}

X-ray photoelectron spectroscopy (XPS) is a powerful tool to investigate the surface properties of materials, including elemental composition and chemical bonding state. The typical set-up for an XPS is shown in Figure 5.19a. An x-ray beam from the source irradiates the sample surface and the emitted electrons are collected and analysed by 
a hemispherical detector. Because of the high energy nature of $x$-rays, they can excite the core electrons of the atoms from their ground state to the vacuum level, becoming free electrons (via the photoelectric effect) and this characteristic energy $E_{b}$ forms the fingerprint of certain elements: 100

$$
E_{b}=E_{0}-\left(E_{k}+\emptyset\right),
$$

where $E_{o}$ is the initial photon energy, $E_{k}$ is the measured kinetic energy by the detector, and $\phi$ is the work function of the spectrometer. With these, we are able to identify elements and measure their corresponding fractions of the target surface.

Ultraviolet photoelectron spectroscopy (UPS) utilizes an identical set-up as XPS, but using a UV source. It can be used for determining the electronic structure of valence states and work function of the target material.

a

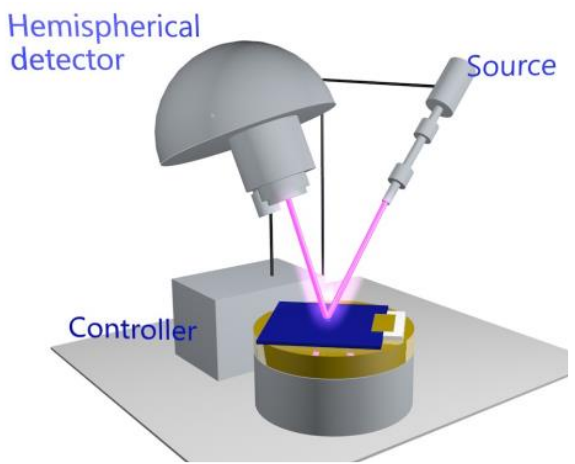

b

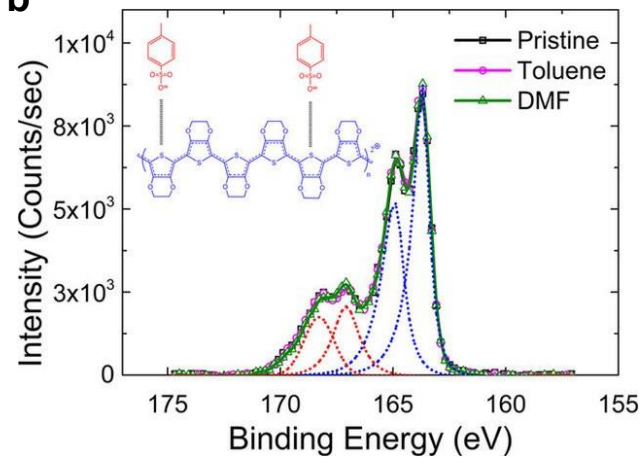

Figure 5.19 | The schematic of XPS/UPS measurement. a, The set-up of XPS/UPS. b, The S(2p) XPS spectra of PEDOT. Figure $\boldsymbol{b}$ is reprinted from Petsagkourakis et al., Sci. Rep. 2016, 6, $30501^{5}$.

In our studies, photoemission measurements were performed using a Scienta ESCA200 spectrometer in an ultrahigh vacuum ${ }^{25,34}$. A monochromatic Al (ka) X-ray and a Helium-discharge lamp were used as the X-ray and ultraviolet sources, respectively. PEDOT thin films were deposited on ITO-coated glass substrates for measurements.

In Paper 2, we focused on the S(2p) XPS spectra of PEDOT samples ${ }^{25}$. An example of $S(2 p)$ XPS spectra is shown in Figure 5.19b. Both PEDOT and its counterion Tos have $S$ element but with different chemical environments. The electronegativity of the atom leads to the formation of two doublets in the spectra, where the high energy one belongs to tosylate and the low energy one originates from PEDOT backbone ${ }^{5}$. The intensity ratio of these two gives the oxidation level of the film surface (Section 4.1.3). In this way, we could characterize the difference between the top and bottom surfaces (Paper 2). In Paper 3, we also used XPS to verify the existence of OTf ( $F$ element) and PEI ( $N$ element) in PEDOT samples ${ }^{34}$. 


\subsubsection{Electrochemical characterization}

Electrochemical characterization is powerful in investigating the redox properties of conducting polymers. Electrochemical tuning of conducting polymer properties offers possibilities for dynamic control of the structural colours in this thesis (Paper 4).

Figure 5.20 shows a typical electrochemical set-up based on three electrodes. The sample we aimed to study (or control sample) is deposited on (or as) the working electrode (W.E.), and it can form an electric circuit with the counter electrode (C.E.). The reference electrode (R.E.) is introduced to ensure that the electrochemical bias between W.E. and C.E. is well calibrated and not deviating from the pre-set voltage. In the standard measurement, we used platinum as the C.E. and silver/silver chloride as the R.E. The electrolyte we used in this thesis was normally aqueous solution of salt (e.g., sodium or potassium chloride).
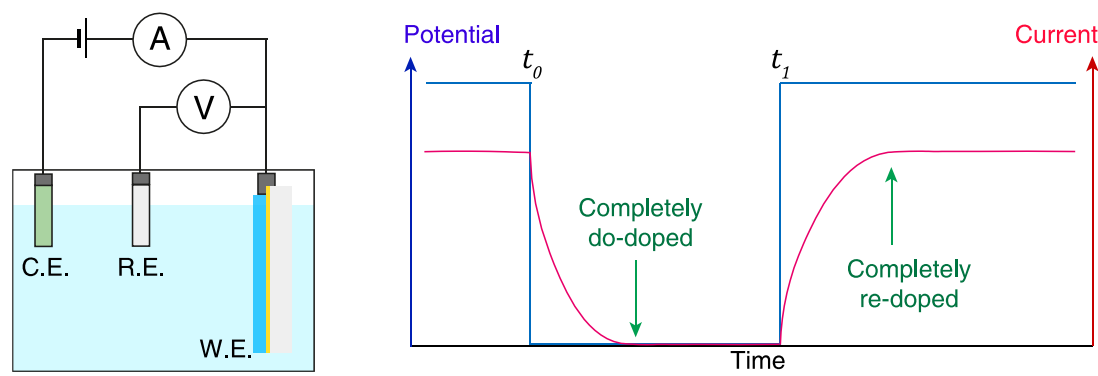

Figure 5.20 | Schematic of a typical electrochemical system and chronocoulometry. The curve for chronocoulometry (right part) is just for schematic illustration.

Chronoamperometry, or chronocoulometry, was used in this thesis, as a method to measure the doping density of conducting polymer films. In chronocoulometry, a fixed bias is applied between the W.E. and the C.E., and the electrical current passing through the device is measured as a function of time (see illustration in the right part of Figure 5.20). In the illustration, when a negative electrochemical bias is applied to reduce a $p$-type conducting polymer at time $t_{0}$, the electric current starts to decay exponentially until it reaches a saturated value (zero). The saturated current indicates that the polymer is completely de-doped. By integrating the current between time $t_{0}$ and $t_{1}$, we can estimate the total charge that was needed for the reduction:

$$
Q=\int_{t_{0}}^{t_{1}} I(t) d t
$$

With the knowledge of thin film area $A$ (immersed into the electrolyte) and thickness $h$, we can calculate the doping density (charge carrier) density $n$ of the film:

$$
n=Q /(A \cdot h) .
$$

It should be noted that this only represents an approach for rough estimation instead of accurate characterization. Hall measurement and spectroscopic ellipsometry would be more reliable methods for charge density determination ${ }^{54,101}$. 
Another application of chronocoulometry is for in operando reflectance measurements. Our set-up for such measurements is depicted in Figure 5.21a, where the sample was placed in a fluidic cell and monitored by an optical microspectroscopy (Section 5.4.4). During the measurement, we could record both in-situ reflectance spectra and optical microscope images simultaneously. In brief, we applied a fixed electro-chemical bias to the conducting polymer W.E., and started to acquire optical spectra and microscope images after around 40 seconds when a saturated current was reached. Examples of results are presented in Figure 5.21b and c, showing clear features of oxidation and reduction for a conducting polymer film on a metal surface (more details in Paper 4).
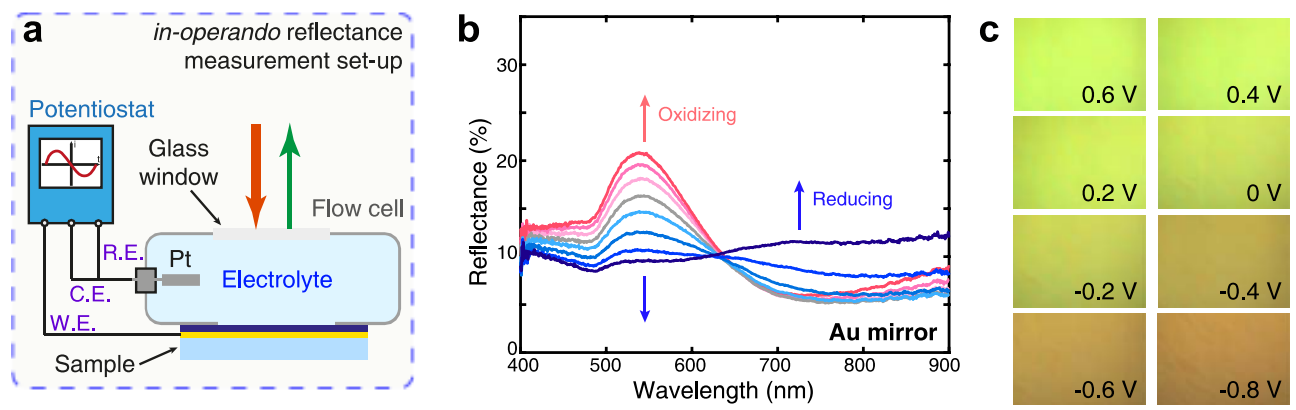

Figure 5.21 | In operando reflectivity measurement by electrochemical switching. a, The set-up for in operando reflectance measurement coupled with an optical microscope. b, Spectra of in operando reflectance measurement at different electrochemical bias. c, Optical microscope images of the sample at different electrochemical bias. The figure is reprinted from Paper 4.

A similar characterization technique that combines chronocoulometry with UV-Vis-NIR spectroscopy was also used in the thesis, with the set-up in Figure $\mathbf{5 . 1 0}$ (more details in Paper 4). The electrochemical workstation we used in this thesis was a potentiostat SP-200 manufactured by BioLogic.

\subsection{References}

$1 \mathrm{Ha}, \mathrm{Y}$. H. et al. Towards a transparent, highly conductive Poly (3, 4-ethylenedioxythiophene). Advanced Functional Materials 14, 615-622 (2004).

2 Mueller, M. et al. Vacuum vapour phase polymerization of high conductivity PEDOT: Role of PEG-PPG-PEG, the origin of water, and choice of oxidant. Polymer 53, 21462151 (2012).

3 Massonnet, N., Carella, A., de Geyer, A., Faure-Vincent, J. \& Simonato, J.-P. Metallic behaviour of acid doped highly conductive polymers. Chemical science $6,412-417$ (2015).

4 Gueye, M. N. et al. Structure and dopant engineering in PEDOT thin films: Practical tools for a dramatic conductivity enhancement. Chemistry of Materials 28, 3462-3468 (2016).

5 Petsagkourakis, I. et al. Structurally-driven enhancement of thermoelectric properties within poly (3, 4-ethylenedioxythiophene) thin films. Scientific reports 6, 30501 (2016). 

studies of the optical properties of doped poly (3, 4-ethylenedioxythiophene): an anisotropic metal. Thin Solid Films 313, 356-361 (1998).

Ugur, A. et al. Low-dimensional conduction mechanisms in highly conductive and transparent conjugated polymers. Advanced Materials 27, 4604-4610 (2015).

Tenhaeff, W. E. \& Gleason, K. K. Initiated and oxidative chemical vapor deposition of polymeric thin films: iCVD and oCVD. Advanced Functional Materials 18, 979-992 (2008).

Wang, X. et al. High electrical conductivity and carrier mobility in oCVD PEDOT thin films by engineered crystallization and acid treatment. Science advances 4, eaat5780 (2018).

Culebras, M., Gómez, C. M. \& Cantarero, A. Enhanced thermoelectric performance of PEDOT with different counter-ions optimized by chemical reduction. Journal of Materials Chemistry A 2, 10109-10115 (2014).

1 Fabretto, M. V. et al. Polymeric material with metal-like conductivity for next generation organic electronic devices. Chemistry of Materials 24, 3998-4003 (2012).

2 Brooke, R. et al. Recent advances in the synthesis of conducting polymers from the vapour phase. Progress in Materials Science 86, 127-146 (2017).

Levermore, P. A., Chen, L., Wang, X., Das, R. \& Bradley, D. D. C. Fabrication of highly conductive poly (3, 4-ethylenedioxythiophene) films by vapor phase polymerization and their application in efficient organic light-emitting diodes. Advanced Materials 19, 2379-2385 (2007).

D'Arcy, J. M. et al. Vapor-phase polymerization of nanofibrillar poly (3, 4-ethylenedioxythiophene) for supercapacitors. ACS nano 8, 1500-1510 (2014).

Evans, D. et al. Structure-directed growth of high conductivity PEDOT from liquid-like oxidant layers during vacuum vapor phase polymerization. Journal of Materials Chemistry 22, 14889-14895 (2012).

Brooke, R., Evans, D., Hojati-Talemi, P., Murphy, P. \& Fabretto, M. Enhancing the morphology and electrochromic stability of polypyrrole via PEG-PPG-PEG templating in vapour phase polymerisation. European polymer journal 51, 28-36 (2014).

Shahnia, S. et al. Towards new fiber optic sensors based on the vapor deposited conducting polymer PEDOT: Tos. Optical Materials Express 9, 4517-4531 (2019). Dall'Acqua, L. et al. Vapour phase polymerisation of pyrrole on cellulose-based textile substrates. Synthetic metals 156, 379-386 (2006).

Hanif, Z., Shin, D., Choi, D. \& Park, S. J. Development of a vapor phase polymerization method using a wet-on-wet process to coat polypyrrole on never-dried nanocellulose crystals for fabrication of compression strain sensor. Chemical Engineering Journal 381, 122700 (2020).

1 Dominguez-Alfaro, A. et al. Tailored Methodology Based on Vapor Phase Polymerization to Manufacture PEDOT/CNT Scaffolds for Tissue Engineering. ACS Biomaterials Science \& Engineering 6, 1269-1278 (2019).

23 high conductivity. Nano Letters 14, 3321-3327 (2014). prepared by vapor phase polymerization for triggered drug delivery: Fabrication and characterization. Electrochimica Acta 269, 560-570 (2018).

Gleason, K. K. CVD Polymers: Fabrication of Organic Surfaces and Devices. (John Wiley \& Sons, 2015).

Chen, S. et al. Unraveling vertical inhomogeneity in vapour phase polymerized PEDOT:

Tos films. Journal of Materials Chemistry A 8, 18726-18734 (2020). 
26 Brooke, R., Fabretto, M., Hojati-Talemi, P., Murphy, P. \& Evans, D. Evidence for 'bottom up'growth during vapor phase polymerization of conducting polymers. Polymer 55, 3458-3460 (2014).

27 Nodora, K. M. A. \& Yim, J. H. Elucidation of the Controversial Layer Growth Mechanism of Vapor Phase Polymerization in the Preparation of Conductive Poly (3, 4ethylenedioxythiophene)-SiO2 Hybrid Films. Advanced Materials Interfaces, 2000046 (2020).

28 Aqrawe, Z. et al. Stretchable Electronics Based on Laser Structured, Vapor Phase Polymerized PEDOT/Tosylate. Polymers 12, 1654 (2020).

29 Brooke, R. et al. Vapor phase synthesized poly (3, 4-ethylenedioxythiophene)-trifluoromethanesulfonate as a transparent conductor material. Journal of Materials Chemistry A 6, 21304-21312 (2018).

30 Shi, H., Liu, C., Jiang, Q. \& Xu, J. Effective approaches to improve the electrical conductivity of PEDOT: PSS: a review. Advanced Electronic Materials 1, 1500017 (2015).

31 Ouyang, J. "Secondary doping" methods to significantly enhance the conductivity of PEDOT: PSS for its application as transparent electrode of optoelectronic devices. Displays 34, 423-436 (2013).

32 Kim, N. et al. Highly conductive PEDOT: PSS nanofibrils induced by solution-processed crystallization. Advanced Materials 26, 2268-2272 (2014).

33 Xia, Y., Sun, K. \& Ouyang, J. Solution-processed metallic conducting polymer films as transparent electrode of optoelectronic devices. Advanced Materials 24, 2436-2440 (2012).

34 Chen, S. et al. Conductive polymer nanoantennas for dynamic organic plasmonics. Nature Nanotechnology 15, 35-40 (2020).

35 Kim, J., Kim, E., Won, Y., Lee, H. \& Suh, K. The preparation and characteristics of conductive poly (3, 4-ethylenedioxythiophene) thin film by vapor-phase polymerization. Synthetic metals 139, 485-489 (2003).

36 Kim, J. Y., Kwon, M. H., Min, Y. K., Kwon, S. \& Ihm, D. W. Self-Assembly and Crystalline Growth of Poly (3, 4-ethylenedioxythiophene) Nanofilms. Advanced Materials 19, 3501-3506 (2007).

37 Zuber, K., Fabretto, M., Hall, C. \& Murphy, P. Improved PEDOT conductivity via suppression of crystallite formation in Fe (III) tosylate during vapor phase polymerization. Macromolecular rapid communications 29, 1503-1508 (2008).

38 Winther-Jensen, B. \& West, K. Vapor-phase polymerization of 3, 4-ethylenedioxythiophene: a route to highly conducting polymer surface layers. Macromolecules $\mathbf{3 7}$, 4538-4543 (2004).

39 Winther-Jensen, B., Chen, J., West, K. \& Wallace, G. Vapor phase polymerization of pyrrole and thiophene using iron (III) sulfonates as oxidizing agents. Macromolecules 37, 5930-5935 (2004).

40 Winther-Jensen, B., Breiby, D. W. \& West, K. Base inhibited oxidative polymerization of 3, 4-ethylenedioxythiophene with iron (III) tosylate. Synthetic metals 152, 1-4 (2005).

41 Shi, W. et al. Micron-thick highly conductive PEDOT films synthesized via self-inhibited polymerization: Roles of anions. NPG Asia Materials 9, e405-e405 (2017).

42 Lee, Y. H., Oh, J., Lee, S.-S., Kim, H. \& Son, J. G. Highly Ordered Nanoconfinement Effect from Evaporation-Induced Self-Assembly of Block Copolymers on In Situ Polymerized PEDOT: Tos. ACS Macro Letters 6, 386-392 (2017).

43 Hojati-Talemi, P., Bächler, C., Fabretto, M., Murphy, P. \& Evans, D. Ultrathin polymer films for transparent electrode applications prepared by controlled nucleation. ACS applied materials \& interfaces 5, 11654-11660 (2013).

44 Schultheiss, A. et al. Water content control during solution-based polymerization: a key to reach extremely high conductivity in PEDOT thin films. Journal of Materials Chemistry C 8, 17254-17260 (2020). 
Franco-Gonzalez, J. F., Rolland, N. \& Zozoulenko, I. V. Substrate-dependent morphology and its effect on electrical mobility of doped poly (3, 4-ethylenedioxythiophene) (PEDOT) thin films. ACS applied materials \& interfaces 10, 29115-29126 (2018). $\mathrm{Li}, \mathrm{B}$. et al. Effects of interfacial modification for vapor phase polymerized PEDOT on glass substrate. Synthetic Metals 260, 116293 (2020).

47 Yewale, R., Damlin, P., Salomäki, M. \& Kvarnström, C. Layer-by-layer approach to engineer and control conductivity of atmospheric pressure vapor phase polymerized PEDOT thin films. Materials Today Communications 25, 101398 (2020).

Losaria, P. M. \& Yim, J.-H. A highly stretchable large strain sensor based on PEDOTthermoplastic polyurethane hybrid prepared via in situ vapor phase polymerization. Journal of Industrial and Engineering Chemistry 74, 108-117 (2019).

Lamont, C. A., Winther-Jensen, O. \& Winther-Jensen, B. A conductive stretchable PEDOT-elastomer hybrid with versatile processing and properties. Journal of Materials Chemistry B 3, 8445-8448 (2015).

Laforgue, A. All-textile flexible supercapacitors using electrospun poly (3, 4ethylenedioxythiophene) nanofibers. Journal of Power Sources 196, 559-564 (2011).

Rehmen, J. et al. Structuring PEDOT Hollow Nanosphere Electrodes for High Specific Energy Li-Metal| Polymer Thin-Film Batteries. ACS Applied Nano Materials 3, 38203828 (2020).

Jia, Y. et al. Efficient enhancement of the thermoelectric performance of vapor phase polymerized poly (3, 4-ethylenedioxythiophene) films with poly (ethyleneimine). Journal of Polymer Science Part B: Polymer Physics 57, 257-265 (2019).

Metsik, J. et al. Growth of poly (3, 4-ethylenedioxythiophene) films prepared by baseinhibited vapor phase polymerization. Journal of Polymer Science Part B: Polymer Physics 52, 561-571 (2014).

Chen, S. et al. On the anomalous optical conductivity dispersion of electrically conducting polymers: ultra-wide spectral range ellipsometry combined with a Drude-Lorentz model. Journal of Materials Chemistry C 7, 4350-4362 (2019).

55 Brooke, R. et al. Controlling the electrochromic properties of conducti
using UV-light. Journal of Materials Chemistry C 6, 4663-4670 (2018).

Barpuzary, D., Kim, K. \& Park, M. J. Two-dimensional growth of large-area conjugated polymers on ice surfaces: high conductivity and photoelectrochemical applications. ACS nano 13, 3953-3963 (2019).

Fabretto, M., Müller, M., Zuber, K. \& Murphy, P. Influence of PEG-ran-PPG surfactant on vapour phase polymerised PEDOT thin films. Macromolecular rapid communications 30, 1846-1851 (2009).

Fabretto, M., Autere, J.-P., Hoglinger, D., Field, S. \& Murphy, P. Vacuum vapour phase polymerised poly (3, 4-ethyelendioxythiophene) thin films for use in large-scale electrochromic devices. Thin solid films 519, 2544-2549 (2011).

Fabretto, M., Zuber, K., Hall, C., Murphy, P. \& Griesser, H. J. The role of water in the synthesis and performance of vapour phase polymerised PEDOT electrochromic devices. Journal of Materials Chemistry 19, 7871-7878 (2009).

Vucaj, N. et al. Vapor phase synthesis of conducting polymer nanocomposites incorporating 2D nanoparticles. Chemistry of Materials 26, 4207-4213 (2014).

61 Edberg, J. et al. Patterning and conductivity modulation of conductive polymers by UV light exposure. Advanced Functional Materials 26, 6950-6960 (2016).

Zuber, K. et al. Influence of Postsynthesis Heat Treatment on Vapor-Phase-Polymerized Conductive Polymers. ACS omega 3, 12679-12687 (2018).

Kim, N. et al. in Conjugated Polymers: Properties, Processing, and Applications (CRC Press Taylor \& Francis Group, 2019).

64 Brooke, R. et al. Greyscale and paper electrochromic polymer displays by UV patterning. Polymers 11, 267 (2019). 
Rodgers, P. \& Heath, J. Nanoscience and technology. Co-Published with Macmillan Publishers Ltd, UK (2009).

Thiruvengadathan, R. et al. Nanomaterial processing using self-assembly-bottom-up chemical and biological approaches. Reports on Progress in Physics 76, 066501 (2013).

7 Cheng, J. Y., Ross, C. A., Smith, H. I. \& Thomas, E. L. Templated self-assembly of block copolymers: top-down helps bottom-up. Advanced Materials 18, 2505-2521 (2006).

68 Hanarp, P., Sutherland, D. S., Gold, J. \& Kasemo, B. Control of nanoparticle film structure for colloidal lithography. Colloids and Surfaces A: Physicochemical and Engineering Aspects 214, 23-36 (2003).

69 Jonsson, M. P., Jönsson, P., Dahlin, A. B. \& Höök, F. Supported lipid bilayer formation and lipid-membrane-mediated biorecognition reactions studied with a new nanoplasmonic sensor template. Nano Letters 7, 3462-3468 (2007).

70 Kang, E. S. H. et al. Strong plasmon-exciton coupling with directional absorption features in optically thin hybrid nanohole metasurfaces. Acs Photonics 5, 4046-4055 (2018).

71 Seidel, Y. E. et al. Stability of nanostructured Pt/glassy carbon electrodes prepared by colloidal lithography. Journal of the Electrochemical Society 155, K50 (2008).

72 Pokki, J. et al. Electroplated porous polypyrrole nanostructures patterned by colloidal lithography for drug-delivery applications. Nanoscale 4, 3083-3088 (2012).

73 Shiran Chaharsoughi, M. et al. Hybrid Plasmonic and Pyroelectric Harvesting of Light Fluctuations. Advanced Optical Materials 6, 1701051 (2018).

74 Fredriksson, H. et al. Hole-mask colloidal lithography. Advanced Materials 19, 42974302 (2007).

75 Cesaria, M. et al. Gold nanoholes fabricated by colloidal lithography: novel insights into nanofabrication, short-range correlation and optical properties. Nanoscale 11, 8416-8432 (2019).

76 Wang, D. \& Möhwald, H. Rapid fabrication of binary colloidal crystals by stepwise spincoating. Advanced Materials 16, 244-247 (2004).

77 Dimitrov, A. S. \& Nagayama, K. Continuous convective assembling of fine particles into two-dimensional arrays on solid surfaces. Langmuir 12, 1303-1311 (1996).

78 Trau, M., Sankaran, S., Saville, D. A. \& Aksay, I. A. Electric-field-induced pattern formation in colloidal dispersions. Nature 374, 437-439 (1995).

79 Zhang, G. \& Wang, D. Colloidal lithography-the art of nanochemical patterning. Chemistry-An Asian Journal 4, 236-245 (2009).

80 Ballo, A., Agheli, H., Lausmaa, J., Thomsen, P. \& Petronis, S. Nanostructured model implants for in vivo studies: influence of well-defined nanotopography on de novo bone formation on titanium implants. International journal of nanomedicine 6, 3415 (2011).

81 Lee, K., Heeger, A. J. \& Cao, Y. Reflectance of polyaniline protonated with camphor sulfonic acid: disordered metal on the metal-insulator boundary. Physical Review B 48, 14884 (1993).

$82 \mathrm{Kim}, \mathrm{N}$. et al. Role of interchain coupling in the metallic state of conducting polymers. Physical Review Letters 109, 106405 (2012).

83 Gentile, M. J., Núñez-Sánchez, S. \& Barnes, W. L. Optical field-enhancement and subwavelength field-confinement using excitonic nanostructures. Nano letters 14, 23392344 (2014).

84 Pettersson, L. A. A., Ghosh, S. \& Inganäs, O. Optical anisotropy in thin films of poly (3, 4-ethylenedioxythiophene)-poly (4-styrenesulfonate). Organic Electronics 3, 143-148 (2002).

85 Tompkins, H. \& Irene, E. A. Handbook of ellipsometry. (William Andrew, 2005). 
Tompkins, H. G. \& Hilfiker, J. N. Spectroscopic Ellipsometry: Practical Application to Thin Film Characterization. (Momentum Press, 2016).

87 Fujiwara, H. Spectroscopic ellipsometry: principles and applications. (John Wiley \& Sons, 2007).

88 Kühne, P., Herzinger, C. M., Schubert, M., Woollam, J. A. \& Hofmann, T. Invited Article: An integrated mid-infrared, far-infrared, and terahertz optical Hall effect instrument. Review of Scientific Instruments 85, 071301 (2014).

89 Kühne, P. et al. Advanced Terahertz Frequency-Domain Ellipsometry Instrumentation forln Situand Ex-Situ Applications. IEEE Transactions on Terahertz Science and Technology 8, 257-270 (2018).

90 Junda, M. M., Phillips, A. B., Khanal, R. R., Heben, M. J. \& Podraza, N. J. Determination of conductivity anisotropy and the role of doping in single walled carbon nanotube thin films with $\mathrm{THz}$ spectroscopic ellipsometry. Carbon 129, 592-597 (2018). Håkansson, A. et al. Effect of (3-glycidyloxypropyl) trimethoxysilane (GOPS) on the electrical properties of PEDOT: PSS films. Journal of Polymer Science Part B: Polymer Physics 55, 814-820 (2017).

Fabiano, S. et al. Poly (ethylene imine) impurities induce n-doping reaction in organic (semi) conductors. Advanced Materials 26, 6000-6006 (2014).

Shanker, R. et al. Noniridescent Biomimetic Photonic Microdomes by Inkjet Printing. Nano Letters 20, 7243-7250 (2020).

94 Dahlin, A. B. et al. High-resolution microspectroscopy of plasmonic nanostructures for miniaturized biosensing. Analytical chemistry 81, 6572-6580 (2009).

95 Müller-Buschbaum, P. The active layer morphology of organic solar cells probed with grazing incidence scattering techniques. Advanced materials 26, 7692-7709 (2014).

96 Gharahcheshmeh, M. H. \& Gleason, K. K. Texture and nanostructural engineering of conjugated conducting and semiconducting polymers. Materials Today Advances 8, 100086 (2020).

97 Miccoli, I., Edler, F., Pfnür, H. \& Tegenkamp, C. The 100th anniversary of the fourpoint probe technique: the role of probe geometries in isotropic and anisotropic systems. Journal of Physics: Condensed Matter 27, 223201 (2015).

98 Ramadan, A. A., Gould, R. D. \& Ashour, A. On the Van der Pauw method of resistivity measurements. Thin solid films 239, 272-275 (1994).

99 Gindl, M., Sinn, G., Gindl, W., Reiterer, A. \& Tschegg, S. A comparison of different methods to calculate the surface free energy of wood using contact angle measurements. Colloids and Surfaces A: Physicochemical and Engineering Aspects 181, 279287 (2001).

100 Xing, K. Z., Fahlman, M., Chen, X. W., Inganäs, O. \& Salaneck, W. R. The electronic structure of poly (3, 4-ethylene-dioxythiophene): studied by XPS and UPS. Synthetic Metals 89, 161-165 (1997).

101 Wu, F. et al. Conductivity enhancement of PEDOT: PSS via addition of chloroplatinic acid and its mechanism. Advanced Electronic Materials 3, 1700047 (2017). 


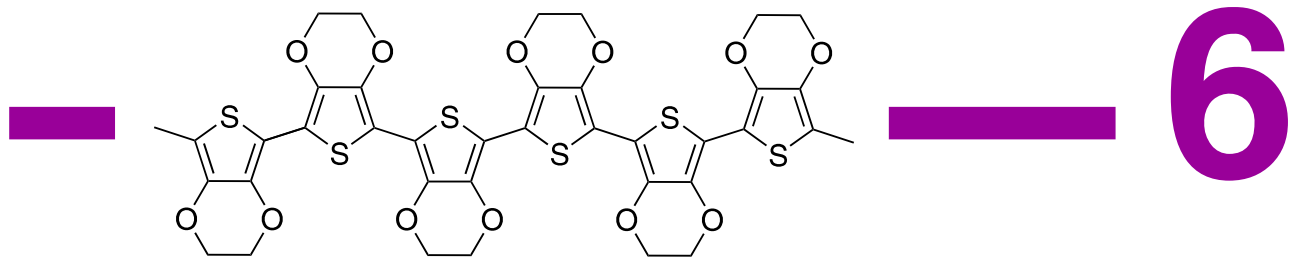

Summary of Appended Papers

The main aim of this thesis was to explore conducting polymers for redox-tunable plasmonics. The optimization of conducting polymer synthesis, and optical studies based on thin films were therefore studied in detail, to meet the requirements of conducting polymer plasmonics. In addition, we developed a new application of conducting polymers for redox-tunable structural colours and successfully made multicolour surfaces and images for proof-of-concept applications.

\section{Contents}

6.1 Optical properties of PEDOT thin films (Paper 1) 103

6.2 Understanding vapour phase polymerization (Paper 2) …..........................105

6.3 Switchable organic nanoantennas (Paper 3) ..............................................107

6.4 Redox-tunable PEDOT structural colours (Paper 4) ...................................109

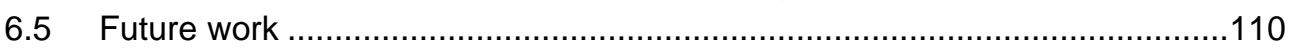

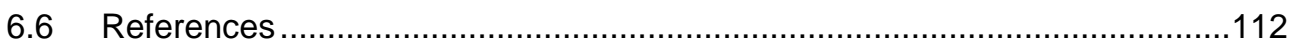

\subsection{Optical properties of PEDOT thin films (Paper 1)}

Lots of efforts have been made to develop new plasmonic materials in order to meet the application requirements of different spectral ranges ${ }^{1,2}$. Certain criteria exist for the excitation of plasmons in materials and complete information on the material optical 
parameters is required (plasmonic regime, see Section 3.3). Reflectivity measurement in the range between UV to MIR combined with Kramers-Kronig transformation has been widely used to derive the permittivity or optical conductivity of conducting polymers ${ }^{3-5}$. However, this method was based on several assumptions to account for regions beyond the characterization limit (e.g., frequencies above UV or below FIR). In addition, normal incidence optical measurements used in this method could hardly provide information on optical anisotropy of the films. Spectroscopic ellipsometry, based on the characterization of polarization and amplitude changes of elliptically polarized light, is therefore more suitable for optical parameter extraction of conducting polymers. The analysis of ellipsometric data strongly relies on specific optical models developed for the material of interest. For conducting polymers, controversies exist for the available optical models.

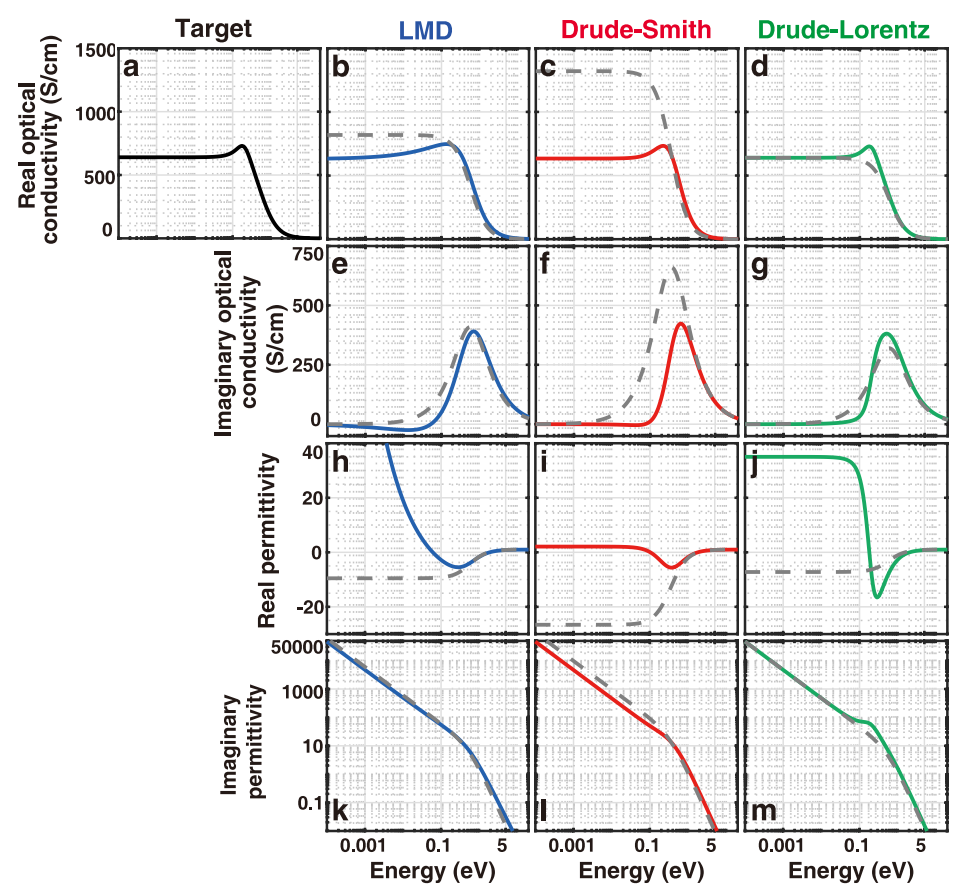

Figure 6.1 | The comparison of three models used in the study. The first column is the target curve obtained from the measurement, and the LMD, the Drude-Smith, and the Drude-Lorentz models are listed in the second, third, and fourth columns. The Drude model curve as a component of each above model is presented by grey dashed lines. The rows from top to bottom are: real optical conductivity (first), imaginary optical conductivity (second), real permittivity (third), and imaginary permittivity (fourth). The figure is reprinted from Reference 6 with permission from the Royal Society of Chemistry.

The most prevailing models in use are the Localization modified Drude (LMD) ${ }^{3}$ and the Drude-Smith (DS) models ${ }^{7}$, developed based on different assumptions (see more details in Section 4.3.5 and 4.3.6). They can both provide reasonably good fittings for 
the same real optical conductivity dispersion, but the derived permittivity dispersions and electrical properties can be dramatically different (Figure 6.1). Therefore, in this paper, we employed ultrawide spectral range ellipsometry (from $0.41 \mathrm{meV}$ to $5.90 \mathrm{eV}$ ) to investigate our model system, PEDOT:Tos thin film. Instead of utilizing the two popular models mentioned above, we proposed a Drude-Lorentz model derived from model dielectric function method (see Section 4.3.6) and the model could successfully fit the experimental data in the optical frequency ranges (Figure 6.2) ${ }^{6}$.

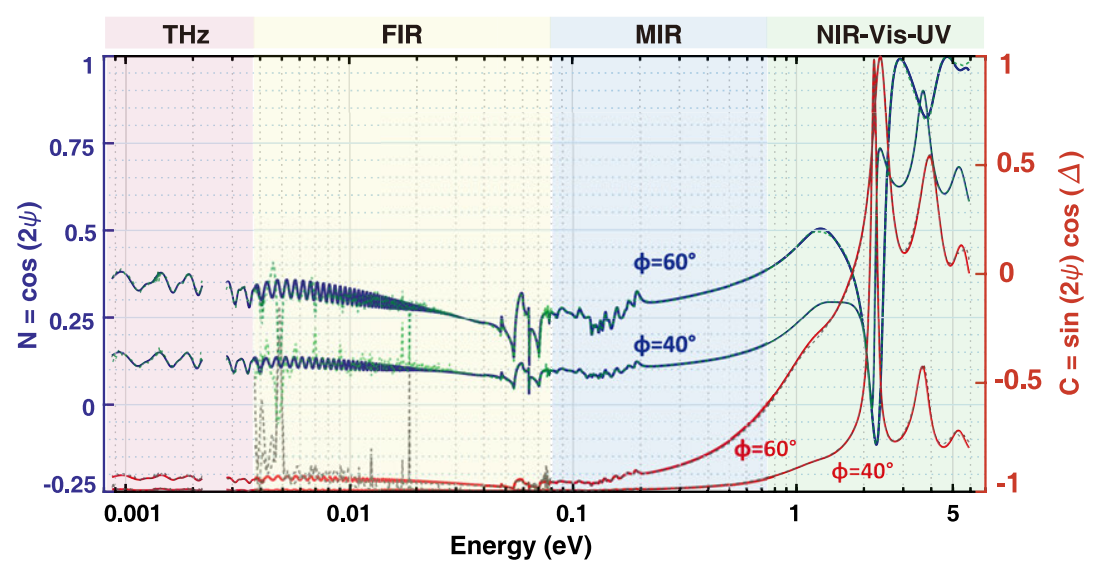

Figure 6.2 | Ellipsometric data and fittings for PEDOT:Tos. The experimental data is plotted in dashed curves while the model fitting is plotted in solid lines. The figure is reproduced from Reference 6 with permission from the Royal Society of Chemistry.

The extracted optical parameter dispersion of PEDOT:Tos exhibited metallic behavior in the spectral range between 1 and $14 \mu \mathrm{m}$, where its real permittivity was negative with a minimum of -44 (see Paper 1$)^{6}$. However, the permittivity ratio, which is defined as the amplitude ratio of real to imaginary permittivity, was smaller than 1 in this range, implying relatively large optical losses. Thus, it might be difficult to fabricate plasmonic structures using this conducting polymer, since the expected plasmonic signals would be weak.

\subsection{Understanding vapour phase polymerization (Paper 2)}

In this paper, we investigated variations in material properties across VPP PEDOT:Tos thin films, which cannot be directly assessed from the ellipsometry study in Paper 1.

PEDOT:Tos films in our study were deposited by vapour phase polymerization (VPP), which is broadly used for polymer films with high performance ${ }^{8,9}$. However, we found that the polymer films obtained by this method were vertically inhomogeneous (in the direction normal to the substrate, or the out-of-plane direction). We used a freestanding film transfer technique (Section 5.1.1) to re-deposit PEDOT films onto new 
substrates with (initial bottom surface-up) or without flipping (or flipping twice, initial top surface-up), as shown in Figure 6.3. We were therefore able to conduct a thorough characterization of the top and bottom surfaces of the VPP PEDOT:Tos films. UV-VisNIR absorption spectroscopy and AFM exhibited no obvious differences between the two samples, indicating that the bulk properties of the two samples were identical.
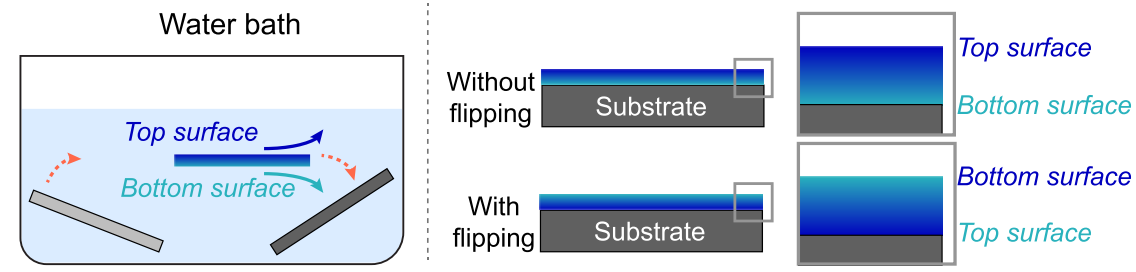

Figure 6.3 | VPP thin films with and without flipping made by free-standing film transfer technique. The initial bottom surface becomes the top in flipped sample, while in non-flipped sample the film configuration does not change (top surface-up). The figure is reproduced from Reference 10 with permission from the Royal Society of Chemistry.

GIWAXS measurements confirmed the existence of vertical stratification in the films. In brief, the crystallinity (also the fraction of edge-on crystallites) was lowest at the bottom surface, but started to increase to a maximum when moving further into the film, and then it decreased towards the top surface of the film. XPS revealed that the bottom surface had an oxidation level of $32 \%$, much higher than that of the top surface (22\%). With these findings, we proposed a possible growth mechanism for VPP, where the imbalance in supply and transport of precursors plays the critical role.
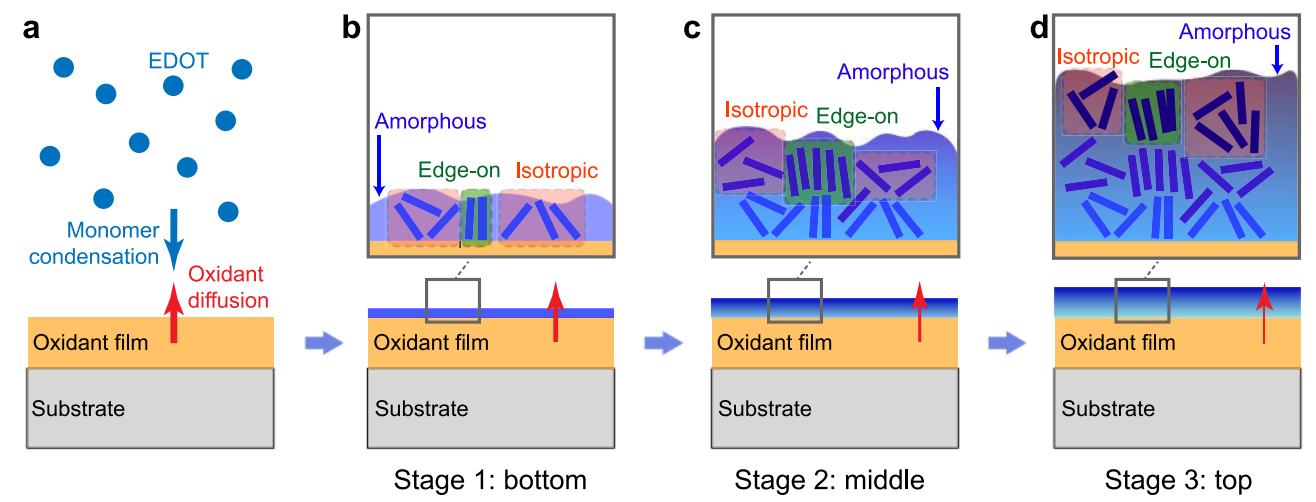

Figure 6.4 | VPP thin films growth mechanism. Three stages exist during the process leading to the vertical inhomogeneity. The figure is reproduced from Reference 10 with permission from the Royal Society of Chemistry.

As indicated in Figure 6.4, there are three characteristic stages in the polymerization process. In the first stage, oxidant film (iron salt) and monomer (EDOT) react at the interface, where an amorphous thin layer forms on top of the gel-like oxidant film. With 
the formation of the first amorphous but solid layer as the template, following layers of PEDOT present higher crystallinity (Stage 2). However, with the increase of PEDOT film thickness, the upward diffusion of oxidant is partially hindered and the doping density (oxidation level) starts to decrease. In the final stage, depletion of oxidants or complete blocking of oxidant transport terminates the polymerization process, leading to the lowest oxidation level and poor crystallinity we observed for the top surface.

Vertical inhomogeneity may limit the conductivity of conducting polymer films. Based on the newly acquired understanding of VPP growth mechanism in this paper, we may eliminate or at least reduce this issue by optimization of polymerization kinetics in the process to balance the supply and transport of the two precursors.

\subsection{Switchable organic nanoantennas (Paper 3)}

Using iron(III) trifluoromethanesulfonate, or $\mathrm{Fe}(\mathrm{OTf})_{3}$, as the oxidant, and optimizing the polymerization kinetics, we could improve the conducting polymer film quality. With concentrated sulfuric acid post-treatment, we obtained PEDOT:Sulf, exhibiting a high electrical conductivity above $5000 \mathrm{~S} / \mathrm{cm}^{11,12}$. By ultrawide spectral range ellipsometry method described in Paper 1, we identified a clear plasmonic regime for this polymer in the wavelength range from about 1 to $4 \mu \mathrm{m}$, showing potential for near-infrared (NIR) plasmonics.
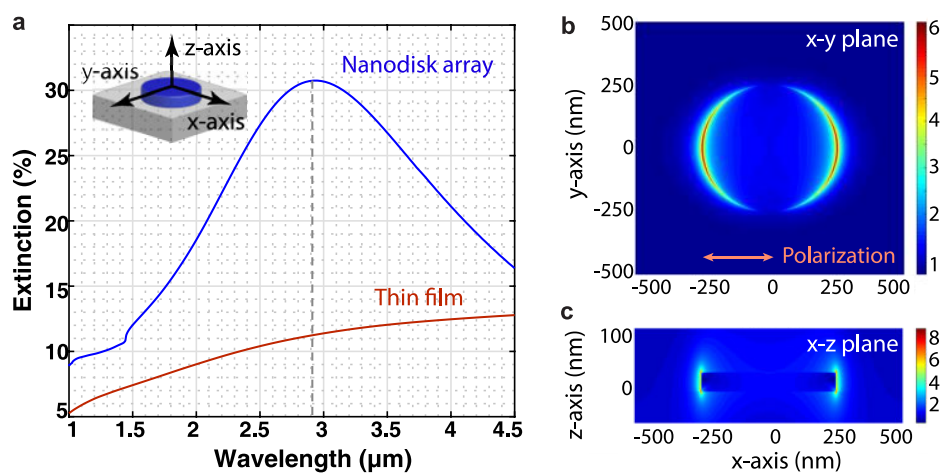

Figure 6.5 | Simulated plasmonic behaviour of PEDOT:Sulf nanodisks. a, Simulated extinction spectra of PEDOT:Sulf nanodisk arrays and thin film. b and $\mathbf{c}$, Simulated near field profiles of PEDOT:Sulf nanodisks in $x-y$ plane (b) and $x-z$ plane (c). The figure is reproduced from Reference 11 with permission from the Springer Nature.

We then performed FDTD simulations using the measured material permittivities of PEDOT:Sulf. The simulated results predicted that nanodisk arrays of the conducting polymers should provide clear extinction peak (see Figure 6.5a) and the peak was attributed to plasmonic resonances (Figure 6.5b and c). Suitable nanodisk diameter was in the order of hundreds of nanometers and thus should be able to be fabricated 
at large scale by colloidal lithography (Section 5.2) ${ }^{13}$. Using self-assembled PS colloids as mask and PMMA as sacrificial layer, we obtained SRO PEDOT nanodisk arrays with well-defined shapes and sizes.

Optical characterization verified that the fabricated polymer nanodisks exhibited clear extinction peaks, which was absent in PEDOT:Sulf films without nanostructures. By altering the diameter and height of the nanodisks, the extinction peak could be redand blue-shifted in a manner that agreed with predictions from FDTD simulations and analytical calculations based on the dipolar polarizability model of oblate spheroids (Section 3.1.2). All these results suggested that the conducting polymer nanostructures provided plasmonic resonance peaks.
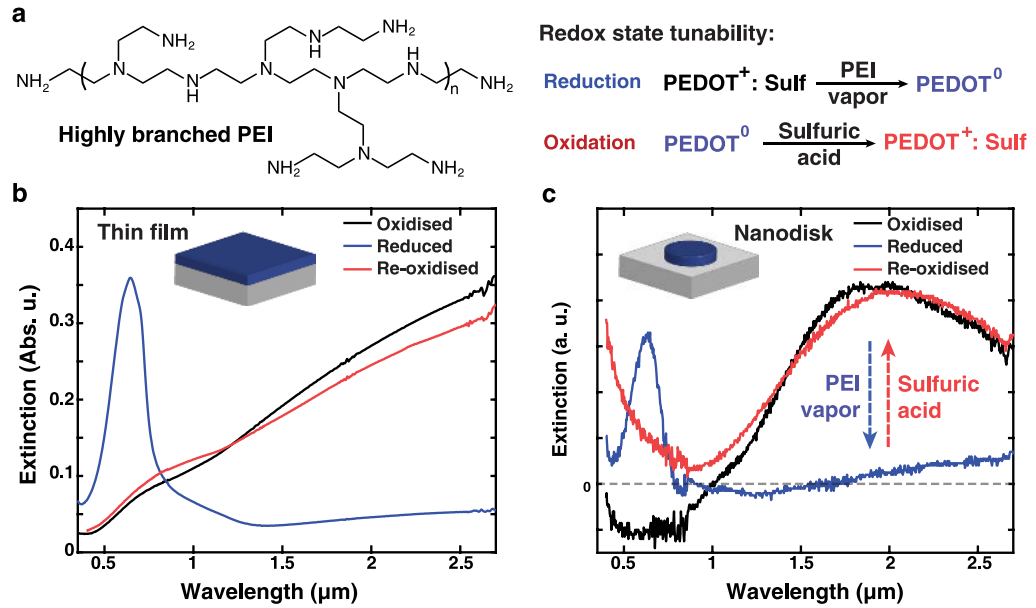

Figure 6.6 | Redox tunability of PEDOT plasmonics. a, Chemical structure of PEI molecules and principles of redox state tunability. $\mathbf{b}$ and $\mathbf{c}$, Experimental extinction spectra of PEDOT thin films and nanodisks by redox tuning. The figure is reprinted from Reference 11 with permission from the Springer Nature.

An important aspect of the study relates to the redox tunability of the conducting polymer, which enabled tuning of the nanodisks after fabrication. Varying the redox states leads to variation of the polymer properties, including possibility to transition between insulating and conducting states (see Section 4.1.4). For our conducting polymer nanodisks, such tuning resulted in drastic changes in the optical behavior between plasmonic and dielectric states. We chose chemical doping methods, using polyethyleneimine (PEI) vapour to de-dope (reduce) the polymer and sulfuric acid to re-dope (oxidize) the polymer (Figure 6.6). The plasmonic resonance could be completely switched off by chemical reduction of the polymer and switched on again by oxidation. The process was reversible and could sustain multiple cycles. As far as we know, this is the first demonstration of plasmonics based on redox-tunable conducting polymers. 


\subsection{Redox-tunable PEDOT structural colours (Paper 4)}

Light-matter interactions enable various approaches to create vivid colours in nature. Typical mechanisms for colour generation include using coloured pigments or dyes ${ }^{14}$, photonic crystals ${ }^{15,16}$, plasmonic naostructures ${ }^{17,18}$, Fabry-Perot cavities ${ }^{22-24}$, and electrochromic devices ${ }^{19-21}$ (see Section 3.4). Making multi-coloured images with the above structures could be challenging, although electron-beam lithography ${ }^{25}$ or multistep photolithography ${ }^{23}$ were demonstrated as possible solutions. In addition to the realization of still images, further dynamic tuning forms another requirement for certain applications ${ }^{26,27}$. In this paper, we demonstrated a facile method to fabricate redoxtunable structural coloured images. By introducing a low-cost UV treatment, complicated pictures could be generated in multiple colours, which also presented tunability via electrochemical control.
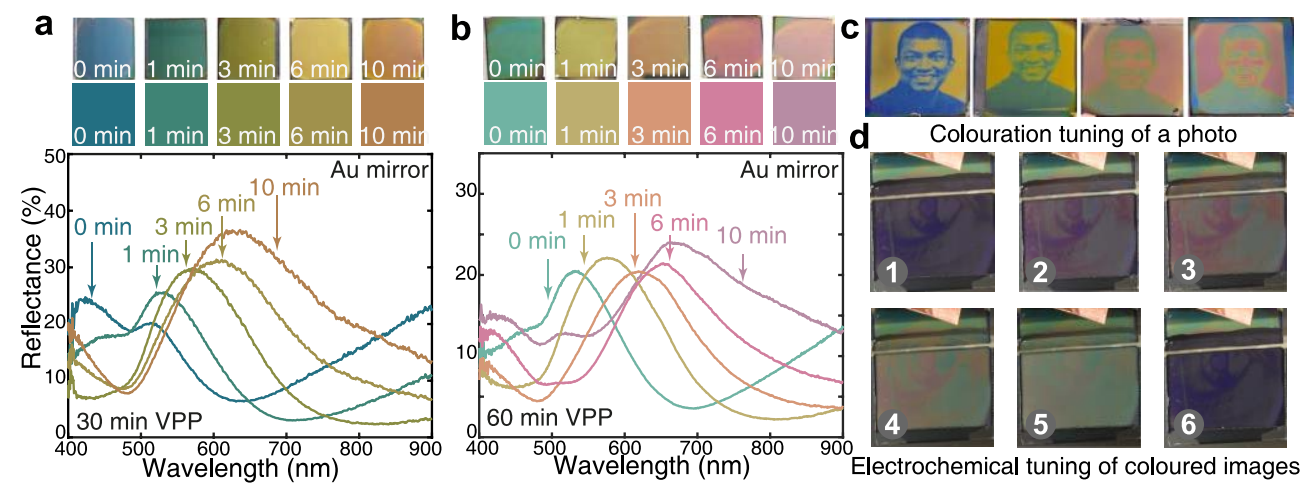

Figure 6.7 | Structural colours of UV-treated PEDOT films. By the optimization of processing parameters during polymerization, devices of colour pixels and complex pictures with multiple colours can be fabricated. The devices are also available for electrochemical tuning.

Using the established methods in Paper 1, we revealed that the permittivity dispersion of PEDOT can vary significantly if an additional UV exposure step is applied to the oxidant before VPP. Three main changes occur during this process: an increase of film thickness, an increase of the real refractive index, and a decrease of absorption coefficient at long wavelength ranges for the final obtained PEDOT films. We therefore implemented these layers by different UV exposure onto a metal mirror and found that they displayed various colours from blue to red, covering almost the whole visible spectrum (Figure 6.7). Additionally, with greyscale photo-masks, we could generate multi-colour images using single UV exposure steps. Numerical simulations and supplementary experiments revealed that the generated colours were a combined effect of material absorption changes and the formation of standing wave between the incident and reflected light of the bottom mirror.

Electrochemical tuning of the devices was explored, showing that the colours could be tuned in a relatively large range from purple to green and red (see Figure 6.7). The 
displayed pictures could be switched off by electrochemical oxidation (positive bias) and switched on by reduction (negative bias). However, the colour ranges in the electrolyte were narrower compared to those in air, as partly attributed to swelling of the polymer. We believe that this new structural colour concept has much room for further optimization, for example, towards new types of smart displays integrated with multiple functions.

\subsection{Future work}

Conducting polymer plasmonics is a new interdisciplinary field that combines nanooptics and organic electronics. PEDOT:Sulf is the first conducting polymer proven to possess a well-defined plasmonic regime and nanodisk arrays of this material showed clear plasmonic resonance behaviour. The primary purpose of developing conducting polymer nano-optics is to use its redox-tunability for specific applications, rather than competing or substituting conventional plasmonic systems based on metals. However, the relatively weak resonance signal of the presented systems is still the primary issue for further research.

There are three potential strategies to overcome this barrier, including synthesis of novel conducting polymers, precise regulation of nano- to microstructures, and use of innovative doping techniques. With great contribution from synthetic chemists, lots of new structures are being designed and synthesized. Promising conducting polymers, including $\mathrm{C}_{12}-\mathrm{PBTTT}{ }^{28}$, PTbT-Tos ${ }^{29}$, and BBL:P(g $\left.\mathrm{g}_{4} 2 \mathrm{~T}-\mathrm{TT}\right)^{30}$, have been reported and investigated by different groups for thermoelectric and bioelectronic applications. With their current excellent conductivity and potentials for further improvements, they would be interesting to study for nano-optics and plasmonics. The properties of conducting polymers are largely affected by their microstructure at different length scales. It has been demonstrated that short-range intermolecular aggregation is the key for high mobility in conjugated polymers ${ }^{31}$. Thus, optimization of microstructure in polymer films may help to further improve their use for plasmonic applications. For VPP demonstrated in Paper 2, multiple factors can influence the final microstructure of the film, including the recipe of oxidants, the pre-treatment of oxidant films, heating and pressure control during polymerization, and post-treatments. In addition, fine control of ordered aggregates induced by off-center spin-coating ${ }^{32}$, mechanical stretching ${ }^{33}$, and solution shearing of blade or brush ${ }^{34-36}$ have shown great capability in achieving highmobility conjugated polymers. Conjugated polymers with large molecular weight or conjugation length may also be advantageous due to enhanced intrachain charge transport ${ }^{30}$. For PEDOT-based materials, the degree of polymerization is mostly $5-20$, ${ }^{37}$ which is far smaller compared to many other conjugated polymers. Thus, the search for high molecular weight PEDOT forms another promising working direction, where a better understanding of polymerization kinetics is desired. For the doping part, more potential topics are waiting to be explored, including the application of all-polymer dopants $^{30}$, ionic liquids ${ }^{38}$, biopolymers ${ }^{39}$, and insulators ${ }^{40}$. It also should be noted that conducting polymers do not have to be conjugated, since non-conjugated radical polymer glass can also show excellent electrical conductivity ${ }^{41}$. 
The secondary challenge for organic plasmonics lies on the design and fabrication of conducting polymer nanostructures. In Paper 3, we utilized colloidal lithography as the main approach to prepare nanodisk arrays with short-range order, for its simplicity and possibility for large-scale fabrication. However, the method is very restrictive in terms of possible patterns that can be generated due to limited ways for the self-assembly of colloidal particles ${ }^{42}$. We attempted to use focused ion beam milling to make polymer nanostructures, but found it challenging due to the polymer being too sensitive to the ion beam. The polymer melted immediately upon its exposure to ions. Thus, finding a suitable way to structure conducting polymers mildly and precisely remains a challenging issue. Instead of top-down methods above, some researchers proposed bottomup ways by using templated substrates ${ }^{43-46}$. However, the growth of conducting polymers is very sensitive to the substrates ${ }^{47}$, and it remains to be investigated if such systems could be suitable for plasmonic applications.

Despite plasmonic features observed in PEDOT nanostructures, the detailed behavior, as well as the physics behind them, is partly unclear. The tertiary challenge for organic plasmonics would be the complete characterization of their properties. For example, the near field distribution of these PEDOT nanodisks was so far only predicted by simulations and we still lack experimental verifications. We demonstrated localized resonances in these nanostructures, but it is still an open question whether surface propagating modes can also be observed, as could be probed by scanning near-field optical microscopy ${ }^{48,49}$ or other techniques.

The last challenge for conducting polymer nano-optics would be its practical applications. Except the "standard" advantage of polymeric materials (low-cost, high material abundance, low-weight, possibility for solution processing, large-scale fabrication, and biocompatibility), the redox-tunability would be the primary advantage of conducting polymers to compete with other nano-optical materials. Some examples of potential applications have been proposed in Paper 3, such as near-infrared displays. I would like to emphasize here on potential bioelectronic applications. The plasmonic spectral range in PEDOT:Sulf is from about 1 to $5 \mu \mathrm{m}$, which covers the second near-infrared biological window (NIR-II, 1 to $1.7 \mu \mathrm{m}^{51}$ ), making these systems relevant for biological sensing and imaging. By implementing bio-functional groups/active sites into polymers via side-chain modification or simply blending, the conducting polymer nanostructures may enable detecting and recording the activities inside body or plants. Neuromorphic computing based on conducting polymer, using its mixed ionic-electronic properties, is emerging as an important direction within materials research. Plasmonics of these mixed ionic-electronic conductors, e.g., PEDOT, might provide a unique approach to realize neuromorphic functions.

The last part of this thesis relates to reflective display devices based on structural colours of PEDOT, forming a different mechanism to create and modulate colours. We have to note some current disadvantages of this device: (i) strong angle-dependence at high degrees (e.g., above $40^{\circ}$ ); (ii) relatively long processing time of UV treatment and VPP, typically at least 1 hour; and (iii) the limited choices of substrates (e.g., Au layer). In addition, detailed mechanism for changes in optical properties, electrical properties, and microstructure induced by UV treatment is not completely known. Future 
work would be focused on three parts: adopting new device structures, modifying oxidant properties, and exploring new applications, such as adaptive (ore responsive) displays combined with solar cells ${ }^{54}$, thermoelectric generators ${ }^{55}$, and triboelectric nanogenerators ${ }^{56}$.

\subsection{References}

1 Naik, G. V., Shalaev, V. M. \& Boltasseva, A. Alternative plasmonic materials: beyond gold and silver. Advanced Materials 25, 3264-3294 (2013).

2 West, P. R. et al. Searching for better plasmonic materials. Laser \& Photonics Reviews 4, 795-808 (2010).

3 Lee, K., Heeger, A. J. \& Cao, Y. Reflectance of polyaniline protonated with camphor sulfonic acid: disordered metal on the metal-insulator boundary. Physical Review B 48, 14884 (1993).

4 Lee, K., Menon, R., Yoon, C. O. \& Heeger, A. J. Reflectance of conducting polypyrrole: Observation of the metal-insulator transition driven by disorder. Physical Review B 52, 4779 (1995).

5 Kim, N. et al. Role of interchain coupling in the metallic state of conducting polymers. Physical Review Letters 109, 106405 (2012).

6 Chen, S. et al. On the anomalous optical conductivity dispersion of electrically conducting polymers: ultra-wide spectral range ellipsometry combined with a Drude-Lorentz model. Journal of Materials Chemistry C 7, 4350-4362 (2019).

7 Smith, N. V. Classical generalization of the Drude formula for the optical conductivity. Physical Review B 64, 155106 (2001).

8 Cho, B. et al. Single-crystal poly (3, 4-ethylenedioxythiophene) nanowires with ultrahigh conductivity. Nano Letters 14, $3321-3327$ (2014).

9 Fabretto, M. V. et al. Polymeric material with metal-like conductivity for next generation organic electronic devices. Chemistry of Materials 24, 3998-4003 (2012).

10 Chen, S. et al. Unraveling vertical inhomogeneity in vapour phase polymerized PEDOT: Tos films. Journal of Materials Chemistry A 8, 18726-18734 (2020).

11 Chen, S. et al. Conductive polymer nanoantennas for dynamic organic plasmonics. Nature Nanotechnology 15, 35-40 (2020).

12 Brooke, R. et al. Vapor phase synthesized poly (3, 4-ethylenedioxythiophene)-trifluoromethanesulfonate as a transparent conductor material. Journal of Materials Chemistry A 6, 21304-21312 (2018).

13 Hanarp, P., Sutherland, D. S., Gold, J. \& Kasemo, B. Control of nanoparticle film structure for colloidal lithography. Colloids and Surfaces A: Physicochemical and Engineering Aspects 214, 23-36 (2003).

14 Comiskey, B., Albert, J. D., Yoshizawa, H. \& Jacobson, J. An electrophoretic ink for all-printed reflective electronic displays. Nature 394, 253-255 (1998).

15 Shanker, R. et al. Noniridescent Biomimetic Photonic Microdomes by Inkjet Printing. Nano Letters 20, 7243-7250 (2020).

16 Teyssier, J., Saenko, S. V., Van Der Marel, D. \& Milinkovitch, M. C. Photonic crystals cause active colour change in chameleons. Nature communications 6, 1-7 (2015).

17 Kristensen, A. et al. Plasmonic colour generation. Nature Reviews Materials 2, 1-14 (2016).

18 Shao, L., Zhuo, X. \& Wang, J. Advanced plasmonic materials for dynamic color display. Advanced Materials 30, 1704338 (2018). 
19 Brooke, R. et al. Infrared electrochromic conducting polymer devices. Journal of Materials Chemistry C 5, 5824-5830 (2017).

20 Brooke, R. et al. Controlling the electrochromic properties of conductive polymers using UV-light. Journal of Materials Chemistry C 6, 4663-4670 (2018).

21 Andersson, P., Forchheimer, R., Tehrani, P. \& Berggren, M. Printable all-organic electrochromic active-matrix displays. Advanced Functional Materials 17, 3074-3082 (2007).

22 Xiong, K. et al. Switchable plasmonic metasurfaces with high chromaticity containing only abundant metals. Nano Letters 17, 7033-7039 (2017).

Xiong, K. et al. Plasmonic metasurfaces with conjugated polymers for flexible electronic paper in color. Advanced Materials 28, 9956-9960 (2016).

Rossi, S. \& Jonsson, M. P. Highly reflective optical nanocavities for structural coloration by combining broadband absorber and Fabry-Pérot effects. Journal of Optics 23, 015001 (2020). Printing with an Electron Beam. Nano Letters (2020).

6 Duan, X. \& Liu, N. Magnesium for dynamic nanoplasmonics. Accounts of Chemical Research 52, 1979-1989 (2019).

7 Duan, X., Kamin, S. \& Liu, N. Dynamic plasmonic colour display. Nature Communications 8, 1-9 (2017).

8 Vijayakumar, V. et al. Bringing conducting polymers to high order: Toward conductivities beyond $10^{5} \mathrm{~S} \mathrm{~cm}^{-1}$ and thermoelectric power factors of $2 \mathrm{mw} \mathrm{m}^{-1} \mathrm{~K}^{-2}$. Advanced Energy Materials 9, 1900266 (2019).

Yuan, D. et al. Quinoid-Resonant Conducting Polymers Achieve High Electrical Conductivity over $4000 \mathrm{~S} \mathrm{~cm}^{-1}$ for Thermoelectrics. Advanced Science 5, 1800947 (2018). $\mathrm{Xu}, \mathrm{K}$. et al. Ground-state electron transfer in all-polymer donor-acceptor heterojunctions. Nature Materials 19, 738-744 (2020).

31 Noriega, R. et al. A general relationship between disorder, aggregation and charge transport in conjugated polymers. Nature Materials 12, 1038-1044 (2013). Yuan, Y. et al. Ultra-high mobility transparent organic thin film transistors grown by an off-centre spin-coating method. Nature Communications 5, 3005 (2014). Wang, Y. et al. A highly stretchable, transparent, and conductive polymer. Science advances 3, e1602076 (2017).

Wang, G. et al. Aggregation control in natural brush-printed conjugated polymer films and implications for enhancing charge transport. Proceedings of the National Academy of Sciences 114, E10066-E10073 (2017).

Worfolk, B. J. et al. Ultrahigh electrical conductivity in solution-sheared polymeric transparent films. Proceedings of the National Academy of Sciences 112, 1413814143 (2015).

Xu, J. et al. Multi-scale ordering in highly stretchable polymer semiconducting films. Nature Materials 18, 594-601 (2019).

37 Elschner, A., Kirchmeyer, S., Lovenich, W., Merker, U. \& Reuter, K. PEDOT: principles and applications of an intrinsically conductive polymer. (CRC press, 2010).

$38 \mathrm{Kim}, \mathrm{N}$. et al. Elastic conducting polymer composites in thermoelectric modules. Nature Communications 11, 1424 (2020).

39 Zhou, S., Quu, Z., Strømme, M. \& Wang, Z. Highly Crystalline PEDOT Nanofiber Templated by Highly Crystalline Nanocellulose. Advanced Functional Materials 30, 2005757 (2020).

40 Gumyusenge, A. et al. Semiconducting polymer blends that exhibit stable charge transport at high temperatures. Science 362, 1131-1134 (2018).

41 Joo, Y., Agarkar, V., Sung, S. H., Savoie, B. M. \& Boudouris, B. W. A nonconjugated radical polymer glass with high electrical conductivity. Science 359, 1391-1395 (2018). 
42 Zhang, G. \& Wang, D. Colloidal lithography-the art of nanochemical patterning. Chemistry-An Asian Journal 4, 236-245 (2009).

43 Yasin, M. N. et al. 3-Dimensionally ordered macroporous PEDOT ion-exchange resins prepared by vapor phase polymerization for triggered drug delivery: Fabrication and characterization. Electrochimica Acta 269, 560-570 (2018).

44 Meng, J. et al. Effects of pore size of reverse opal structured PEDOT films on their electrochromic performances. Organic Electronics 50, 16-24 (2017).

45 Jung, Y. S., Jung, W., Tuller, H. L. \& Ross, C. A. Nanowire conductive polymer gas sensor patterned using self-assembled block copolymer lithography. Nano Letters $\mathbf{8}$, 3776-3780 (2008).

46 Wu, P., Guo, J., Jiang, K., Wang, J. \& Jiang, L. Direct Water-Writing/Electroerasing Pattern on PEDOT Inverse Opals. Advanced Functional Materials 29, 1808473 (2019).

47 Taggart, D. K., Yang, Y., Kung, S.-C., Mclntire, T. M. \& Penner, R. M. Enhanced thermoelectric metrics in ultra-long electrodeposited PEDOT nanowires. Nano Letters 11, 125-131 (2011).

48 Chen, J. et al. Optical nano-imaging of gate-tunable graphene plasmons. Nature 487, 77-81 (2012).

49 Fei, Z. et al. Gate-tuning of graphene plasmons revealed by infrared nano-imaging. Nature 487, 82-85 (2012).

50 Poddubny, A., lorsh, I., Belov, P. \& Kivshar, Y. Hyperbolic metamaterials. Nature Photonics 7, 948-957 (2013).

51 Yang, Q. et al. Rational design of molecular fluorophores for biological imaging in the NIR-II window. Advanced Materials 29, 1605497 (2017).

52 Hirsch, L. R. et al. Nanoshell-mediated near-infrared thermal therapy of tumors under magnetic resonance guidance. Proceedings of the National Academy of Sciences 100, 13549-13554 (2003).

53 Zheludev, N. I., Prosvirnin, S. L., Papasimakis, N. \& Fedotov, V. A. Lasing spaser. Nature photonics 2, 351-354 (2008).

54 Park, N.-G. Perovskite solar cells: an emerging photovoltaic technology. Materials today 18, 65-72 (2015).

55 Zhao, D., Fabiano, S., Berggren, M. \& Crispin, X. Ionic thermoelectric gating organic transistors. Nature communications 8, 1-7 (2017).

56 Wu, C., Wang, A. C., Ding, W., Guo, H. \& Wang, Z. L. Triboelectric nanogenerator: a foundation of the energy for the new era. Advanced Energy Materials 9, 1802906 (2019). 


\section{Part II}

\section{Scholarly Articles}





\section{Papers}

The papers associated with this thesis have been removed for copyright reasons. For more details about these see:

http://urn.kb.se/resolve?urn=urn:nbn:se:liu:diva-173352 


\section{FACULTY OF SCIENCE AND ENGINEERING}

Linköping Studies in Science and Technology, Dissertation No. 2107, 2021

Department of Science and Technology

Linköping University

SE-581 83 Linköping, Sweden

\section{www.liu.se}

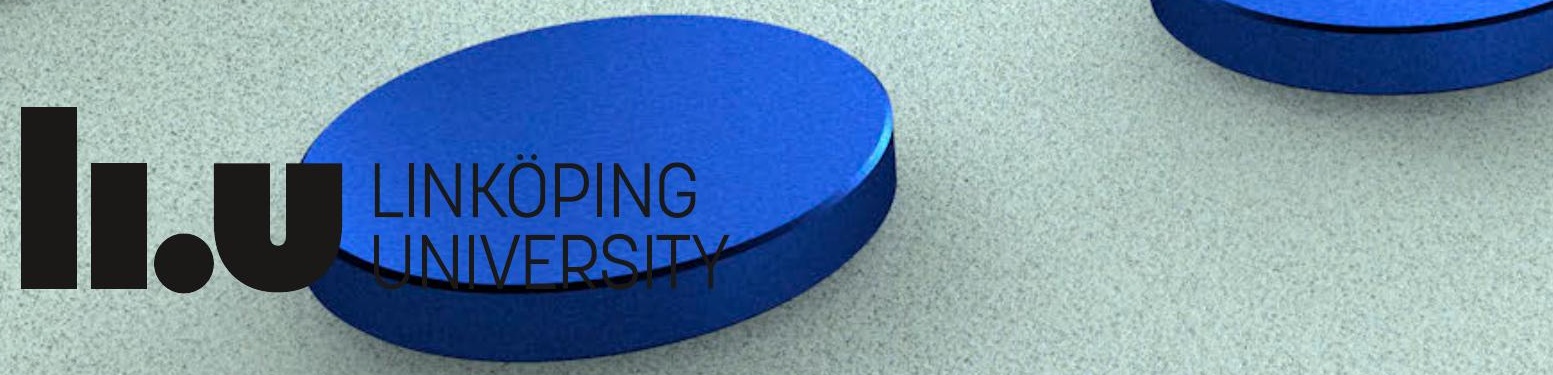

